\title{
Dietary manipulation of fat metabolism in relation to obesity and insulin resistance
}

Citation for published version (APA):

Konings, E. (2013). Dietary manipulation of fat metabolism in relation to obesity and insulin resistance. [Doctoral Thesis, Maastricht University]. Uitgeverij BOXPress. https://doi.org/10.26481/dis.20130607ek

Document status and date:

Published: 01/01/2013

DOI:

10.26481/dis.20130607ek

Document Version:

Publisher's PDF, also known as Version of record

\section{Please check the document version of this publication:}

- A submitted manuscript is the version of the article upon submission and before peer-review. There can be important differences between the submitted version and the official published version of record.

People interested in the research are advised to contact the author for the final version of the publication, or visit the DOI to the publisher's website.

- The final author version and the galley proof are versions of the publication after peer review.

- The final published version features the final layout of the paper including the volume, issue and page numbers.

Link to publication

\footnotetext{
General rights rights.

- You may freely distribute the URL identifying the publication in the public portal. please follow below link for the End User Agreement:

www.umlib.nl/taverne-license

Take down policy

If you believe that this document breaches copyright please contact us at:

repository@maastrichtuniversity.nl

providing details and we will investigate your claim.
}

Copyright and moral rights for the publications made accessible in the public portal are retained by the authors and/or other copyright owners and it is a condition of accessing publications that users recognise and abide by the legal requirements associated with these

- Users may download and print one copy of any publication from the public portal for the purpose of private study or research.

- You may not further distribute the material or use it for any profit-making activity or commercial gain

If the publication is distributed under the terms of Article $25 \mathrm{fa}$ of the Dutch Copyright Act, indicated by the "Taverne" license above, 
Dietary manipulation of fat metabolism in relation to

\author{
obesity and insulin resistance
}




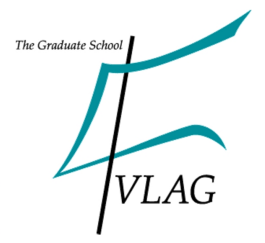

The study presented in this thesis was performed within NUTRIM, School for Nutrition, Toxicology and Metabolism which participates in the Graduate School VLAG (Food Technology, Agrobiotechnology, Nutrition and Health Sciences), accredited by the Royal Netherlands Academy of Arts and Sciendes.

Cover design: Sanne Peeters / Annemieke den Boer

Layout: $\quad$ Ellen Konings

Printed by: $\quad$ Proefschriftmaken.nl || Uitgeverij BOXPress

(C) Copyright Ellen Konings, Maastricht 2013

ISBN 978-90-8891-594-9 


\section{Dietary manipulation of fat metabolism in relation to}

\section{obesity and insulin resistance}

\section{PROEFSCHRIFT}

ter verkrijging van de graad van doctor

aan de Universiteit Maastricht, op gezag van de Rector Magnificus, Prof. L.L.G. Soete volgens het besluit van het College van Decanen,

in het openbaar te verdedigen

op vrijdag 7 juni 2013 om 14.00 uur

door

\section{Ellen Konings}

geboren te Hasselt, 6 november 1985 


\section{Promotores}

Prof. dr. Ellen E. Blaak

Prof. dr. Edwin C. Mariman

\section{Beoordelingscommissie}

Prof. dr. Wim H. Saris (voorzitter)

Prof. dr. Karine Clément (University Pierre et Marie Curie-Paris 6)

Dr. Jogchum Plat

Prof. dr. Casper G. Schalkwijk

Prof. dr. Annemie M. Schols

The research in this thesis was supported by grants from the Dutch Diabetes Research Foundation (DFN 98.901, DFN 2000.00.020 and DFN 2006.00.019), the NutriGenomics Consortium, Top Institute Food and Nutrition and Tate and Lyle Health \& Nutrition Sciences.

Financial support by the Netherlands Association of the Study of Obesity (NASO) and Novo Nordisk B.V. for the publication of this thesis is gratefully acknowledged.

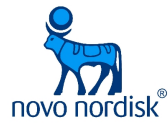




\section{Contents}

Chapter $1 \quad$ General Introduction

Chapter 2 Expression of genes involved in lipid metabolism in impaired

glucose tolerant men: impact of insulin stimulation and weight loss

Chapter 3 Calorie restriction-like effects of 30 days resveratrol supplementation on energy metabolism and metabolic profile in obese humans

Chapter 4 The effects of 30 days resveratrol supplementation on adipose tissue morphology and gene expression patterns in obese men

Chapter 5 Polyunsaturated fatty acids acutely affect triacylglycerol-derived skeletal muscle fatty acid uptake and increases postprandial insulin sensitivity.

Chapter 6 The effect of polydextrose and soluble corn fiber on energy metabolism, metabolic profile and appetite control in overweight men and women

Chapter 7 General Discussion

Summary - Samenvatting 



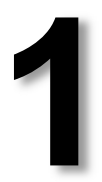

General Introduction 


\section{OBESITY AND INSULIN RESISTANCE}

The global epidemic of overweight and obesity is rapidly becoming a major public health problem in many parts of the world, with a prevalence of at least 500 million people worldwide (1). In addition to genetic predisposition, an inactive lifestyle as well as calorie-dense food consumption are the main causes of excessive weight gain $(2,3)$. Together with the obesity epidemic, the prevalence of related disorders, such as the metabolic syndrome, non-alcoholic fatty liver disease, and type 2 diabetes mellitus (T2DM), is also rising. Insulin resistance plays a crucial role in the pathogenesis of all these disorders (4). Insulin resistance is defined as an impaired sensitivity to insulin of its main target organs, i.e. skeletal muscle, liver and adipose tissue. Since skeletal muscle is a key metabolic tissue, accounting for $70-80 \%$ of total glucose disposal under postprandial conditions, defects in insulin action in this tissue are central to the pathogenesis of $\operatorname{T2DM}(5,6)$. A reduction of insulinmediated peripheral glucose uptake produces an elevation of glucose concentrations predominantly in the postprandial state (impaired glucose tolerance, IGT), whereas an impaired insulin-mediated suppression of hepatic glucose output may importantly contribute to fasting hyperglycemia (impaired fasting glucose, IFG). Subjects with IFG and/or IGT have a 5-12 times increased risk for developing T2DM compared to individuals with normal fasting and postprandial glucose (7). But most obese, insulin-resistant individuals do not develop hyperglycemia. Under normal conditions, the pancreatic islet $\beta$-cells increase insulin release sufficiently to overcome the reduced insulin action, thereby maintaining normal glucose tolerance $(8,9)$. However, when insulin-producing pancreatic $\beta$-cells can no longer compensate for the decreased tissue insulin sensitivity, glucose homeostasis deteriorates and the pre-diabetic state and eventually frank T2DM develop (10).

\section{INSULIN ACTION}

The metabolic actions of insulin with respect to glucose metabolism are maintaining glucose homeostasis by promoting glucose uptake in skeletal muscle and suppressing glucose production in the liver. In skeletal muscle, insulin binds to the extracellular subunit of the insulin receptor. This causes a conformational change in the $\beta$-subunit, which has intrinsic tyrosine kinase activity, resulting in auto-phosphorylation of insulin receptor tyrosine residues and enhanced tyrosine kinase activity of the receptor. The activated receptor phosphorylates the insulin receptor substrate (IRS) proteins. When phosphorylated, IRS-1 activates phosphatidylinositol 3-kinase (PI3-K). Insulin-activated PI3-K regulates the activation of PKB/Akt and atypical PKC isoforms $\lambda / \zeta$, which results in GLUT4 translocation from its intracellular pool to the plasma membrane and glucose transport into the cell (Figure 1) $(11,12)$. In adipose tissue, insulin is antilipolytic, 
where it inhibits the release of fatty acids from adipocytes by decreasing the activity of hormone-sensitive lipase (HSL) and adipose triglyceride lipase (ATGL) (13). Insulin also stimulates lipoprotein lipase (LPL) activity to enhance triacylglycerol (TAG) clearance (14). In the liver, insulin decreases the release of glucose from the liver by inhibiting hepatic glycogenolysis (glycogen breakdown to glucose) and the expression of key gluconeogenic enzymes (15).

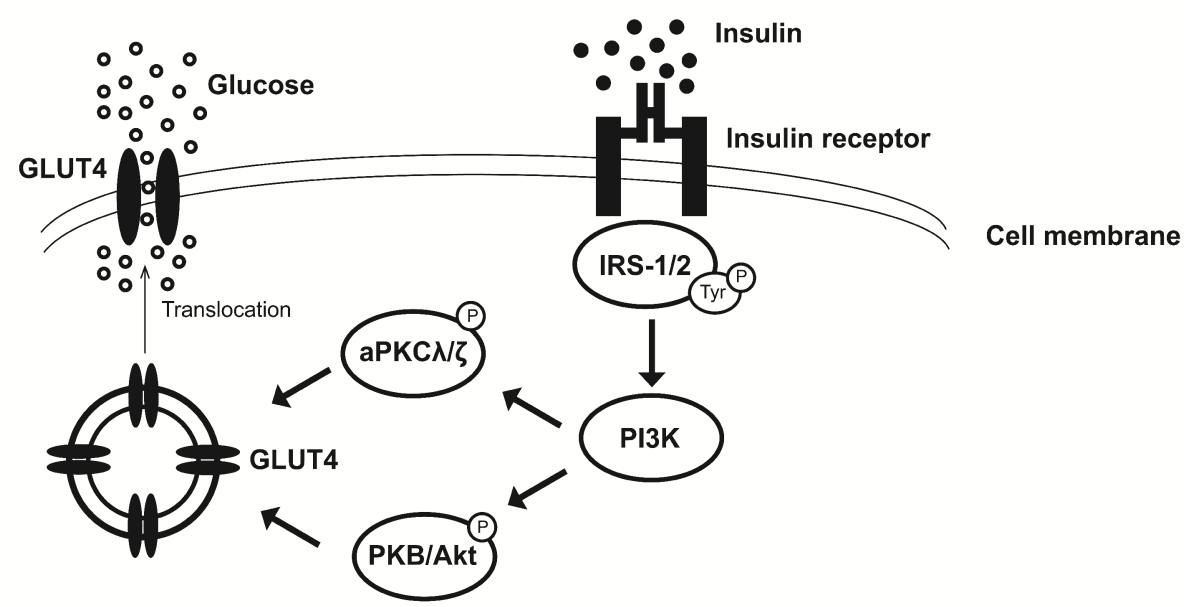

Figure 1. Simplified overview of the insulin signaling cascade involved in stimulating glucose transport. See text above for more information.

\section{FAT METABOLISM IN THE HEALTHY STATE}

Major organs involved in fat metabolism are adipose tissue, skeletal muscle and the liver (Figure 2). Dietary fat that is absorbed by the enterocytes can be repackaged into chylomicron (CM) lipoprotein particles for distribution to the body tissues, stored within the enterocyte or stored or oxidized in other tissues. Once absorbed and released into the blood, dietary fatty acids carried in CM deliver lipids to different parts of the body to be metabolized. The release of fatty acids from CMTAG occurs through the activity of LPL, which is bound to the capillary endothelium of major organs in the body (16). LPL activity is regulated according to the nutritional state in a tissue-specific manner according to the needs of the tissue for fatty acids. In the postprandial state, LPL is activated in adipose tissue and downregulated in skeletal muscle to direct fatty acids towards adipose tissue for storage. The reverse is true in the fasted state since it has been shown that LPL is activated in skeletal muscle, while it is suppressed in adipose tissue to direct fatty acids to skeletal muscle for oxidation (17). CM-derived fatty acids are taken up by muscle and adipose tissue to a greater extent than very low-density lipoprotein 
(VLDL)-TAG fatty acids derived from the liver. This preferential uptake of CM as opposed to VLDL fatty acids appears strongest in the early postprandial period (18). Adipose tissue is the main storage depot for lipids in the form of TAG. The buffer capacity of adipose tissue refers to the capability of clearing circulating $\mathrm{CM}$ and VLDL-TAG in the postprandial state, while suppressing the release of fatty acids into the circulation by means of a reduced endogenous lipolysis (19). Lipolysis of adipose tissue, which liberates free fatty acids (FFA) from adipocytes, plays another important regulatory role in conditions of increased energy demand. FFA are an important fuel for skeletal muscle, in particular when chylomicron and VLDL-TAG levels decrease in the post-absorptive state (20). Once entering the muscle or liver, fatty acids are directed towards either synthesis of lipid metabolites or mitochondrial $\beta$-oxidation (Figure 2). Disturbances in fat metabolism in either of these organs can contribute to the development of insulin resistance.

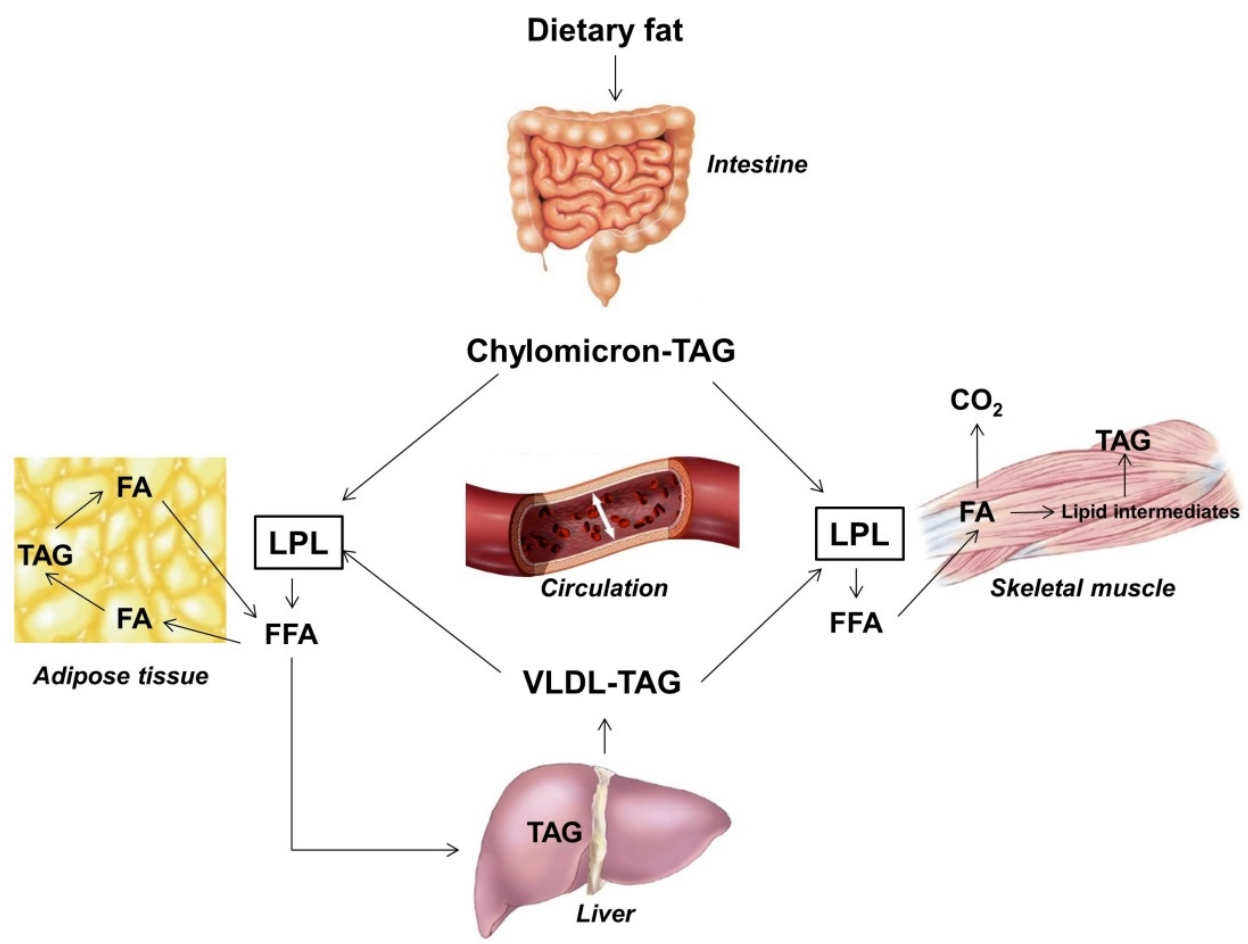

Figure 2. Simplified overview of the exogenous and endogenous pathways of fat metabolism.

\section{FAT METABOLISM IN THE INSULIN RESISTANT STATE}

As indicated above, adipose tissue plays a crucial role in buffering the flux of fatty acids in the circulation in the postprandial period. In a state of nutritional overload, the adipocyte increases in size until it eventually reaches a state where it can no 
longer store additional lipids. When the maximum capacity of adipose tissue expansion is reached, lipids will remain in the circulation resulting in an increased flux and storage in non-adipose tissues (21). A dysbalance between fatty acid uptake and oxidation may lead to ectopic lipid accumulation to occur in tissues like the liver, skeletal muscle and the $\beta$-cells in the pancreas (22). This dysbalance may be explained by an increased supply of lipids from the circulation or a reduced regulation and/or capacity to oxidize fatty acids (Figure 3 ). It is still unclear how the accumulation of lipids becomes toxic to the cell, and much work has focused on identifying which lipid species are detrimental to insulin signaling and other metabolic processes. It has been suggested that TAGs themselves are neutral lipid species, while lipid intermediates such as diacylglycerols (DAGs), ceramides, and fatty acyl-CoAs are able to inhibit insulin signaling pathways and promote insulin resistance (23).

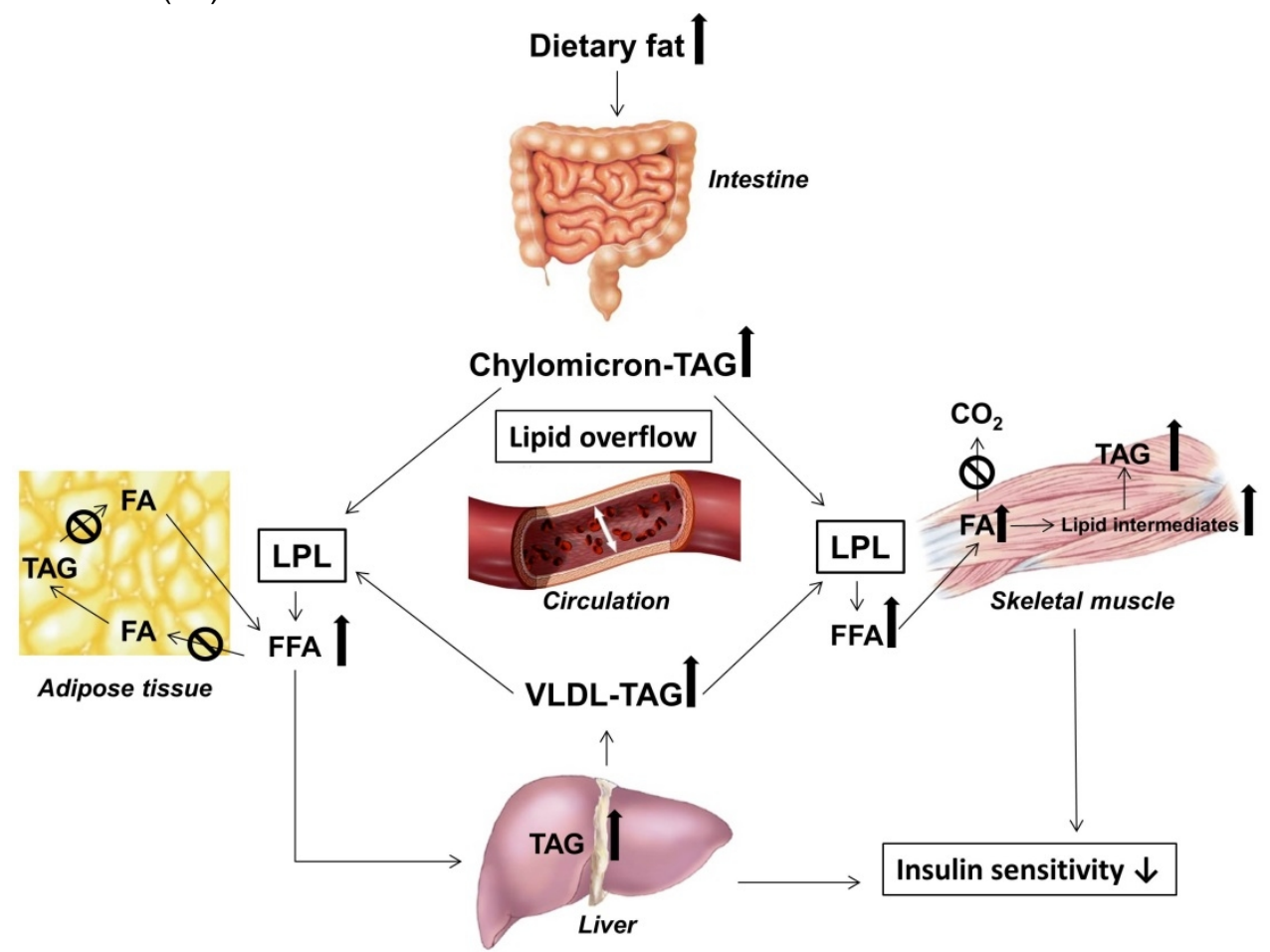

Figure 3. Disturbances in the exogenous and endogenous pathways of fat metabolism may cause lipid overflow. Lipid overload by obesity or a high fat diet contributes to ectopic fat storage. Loss of the normal buffer capacity of the adipose tissue may increase the flux of fatty acids and triacylglycerols (TAG) into the circulation. Fatty acids are taken up in peripheral tissues like skeletal muscle and the liver. An impaired fat oxidation in these tissues may direct these lipids towards storage and accumulation of lipid metabolites, which may lead to the development of insulin resistance. 
To better understand the inter-organ crosstalk in fat metabolism and the relation with insulin resistance, the next paragraphs will discuss the role of adipose tissue function as well as the role of skeletal muscle fat metabolism in the development of insulin resistance.

\section{ADIPOSE TISSUE FUNCTION AND DYSFUNCTION}

Adipose tissue stores TAG during dietary energy consumption and releases fatty acids when energy expenditure exceeds energy intake during fasting (24). In humans, dietary fatty acids are taken up by adipose tissue by extracellular lipolysis of CM- and VLDL-TAG by LPL (25). In adipose tissue, the major activator of LPL is insulin (26). Therefore, in the fed state, LPL is activated in white adipose tissue and fatty acids will be directed to adipose tissue for esterification and storage in time of energy excess (17). However, a significant proportion of the fatty acids from exogenous lipolysis can also spillover in the circulation and enter the plasma FFA pool (27). The process of fatty acid trapping in adipose tissue has shown to be dependent on the concentration gradient of fatty acids between adipocytes and plasma, generated by the activity of the esterification pathway of fatty acids and by intracellular lipolysis $(19,27,28)$. The principal role of adipose tissue lipolysis is to provide other tissues with fatty acids in case of increased energy demand. Lipolysis of TAG to fatty acids and glycerol requires three consecutive steps: the enzyme ATGL catalyzes the initial step of lipolysis, converting TAG to DAG (29); HSL is mainly responsible for the hydrolysis of DAG to monoacylglycerols (MAG) and MG lipase (MGL) hydrolyzes MAG (30). Lipolysis requires that the different lipases can access the triglycerides, which are coated by perilipin proteins surrounding the lipid droplet (31). Perilipin A normally prevents lipolysis of TAG by surrounding the lipid droplet, preventing the access of lipases (32). ATGL requires a coactivator protein, comparative gene identification-58 (CGI-58), for full hydrolase activity (33). Recently, Yang et al. discovered a specific peptide inhibitor of ATGL, G0G1 switch protein 2 (GOS2). G0S2 expression is very low in adipose tissue during fasting but increases after feeding (34). Lipolysis is under strong hormonal regulation. During fasting, catecholamines are major hormones that stimulate lipolysis. Catecholamines like noradrenaline and adrenaline can regulate lipolysis by binding to different adrenoreceptor subtypes ( $\beta 1-3$ and $\alpha 2)$ that are coupled with membrane-bound $G$ proteins. In the fed state, insulin is the most potent antilipolytic hormone. Insulin can activate phosphodiesterase, reducing the intracellular levels of cAMP, thus reducing the activation of HSL (35). 


\section{Lipid overflow: circulating FFA}

In the obese insulin resistant state, elevated FFA concentrations are thought to arise from an increased adipose tissue mass. Although an increased adipose tissue mass can deliver more FFA into the circulation, the differences in FFA concentrations between obese and lean subjects are generally modest and appear not to be proportionally increased to the size of fat mass (36). In contrast, the rate of FFA release per unit fat mass is considerably decreased in the obese. It has been shown that the rate of FFA release per unit fat mass is almost halved in obese subjects compared with lean in the basal state (37). Furthermore, a study performed in 106 lean, overweight and obese non-diabetic subjects with varying levels of adiposity (7-56\% body fat) showed a decrease in the rate of FFA release from adipose tissue with increasing fat mass. However, this downregulation in the rate of FFA release per unit of body fat does not completely compensate for the increase in total body fat, so whole body FFA rate of appearance increased with increasing amounts of body fat (38). Moreover, the reduced lipolysis per unit fat mass has been shown to be associated with downregulation of the expression of key enzymes of fat mobilization, HSL and ATGL in obese subjects (39-41). This suggests that hyperinsulinemia often present in the obese state may cause a downregulation of FFA release to prevent an excessive fatty acid outflow and worsening of the insulin resistant state (42). Furthermore, it is also argued that adipose tissue lipolysis, normally suppressed by insulin, becomes insulin resistant. This has been shown by insulin infusion studies in which insulin resistance of lipolysis was observed in obese subjects $(37,43)$. However, clamp studies may be misleading because in subjects with insulin resistance, plasma insulin concentrations are elevated and the so-called insulin resistance of adipose tissue lipolysis may simply reflect an adaptation to high ambient insulin concentrations.

Increased systemic FFA concentrations may also be achieved by a reduced fatty acid trapping of the adipose tissue after extracellular lipolysis of circulating TAG by LPL. However, two recent studies using a dual fatty acid stable isotope technique showed that fatty acid spillover derived from the hydrolysis of CM-TAG was not different between BMI matched insulin resistant and control subjects (18, 44). These data indicate that an increased spillover from TAG-derived FA most likely does not contribute to an elevation in circulating FFA in the insulin resistant state.

\section{Lipid overflow: circulating TAG}

Elevated TAG concentrations in both the fasting and postprandial state may be more strongly associated with insulin resistance as compared to increased FFA concentrations. TAG concentrations have been reported to be increased in the obese insulin resistant state, which may be attributed to an increased liver VLDL production or by impairments in TAG clearance from the circulation (18, 45-47). 
A reduced adipose tissue TAG clearance has been reported in obesity due to a lower functional LPL activity per unit fat mass in combination with a reduced postprandial upregulation of adipose tissue LPL by insulin $(45,48)$. Furthermore, in obese subjects it has been shown that clearance of VLDL-TAG by adipose tissue was decreased after an overnight fast, whilst in the postprandial state CM-TAG clearance decreased and VLDL-TAG seemed to be better able to compete with chylomicrons for clearance by $\operatorname{LPL}(39,45)$. These data indicate that in obesity CM-TAG are less effective substrates for LPL than VLDL-TAG possibly due to a greater VLDL particle size reflecting an increased lipid content (49). The studies mentioned here compared obese with lean subjects, which does not necessarily imply that these data are representative for the insulin resistant state per se. Indeed, comparing insulin resistant and control subjects with no or only a slight difference in fat mass did not show a difference in CM-TAG extraction across adipose tissue or gives, although CM-TAG extraction was not directly measured, comparable CM-TAG concentrations in the circulation $(44,50)$. Obviously, there is a need for more studies in this area covering the role of TAG concentrations in the insulin resistant state.

\section{Impaired adipocyte differentiation}

An increase in adipose tissue lipid buffering capacity may, on one hand, be achieved by an increased expansion of mature adipocytes (hypertrophy) and on the other hand by recruiting new adipocytes (hyperplasia). In adult man, hypertrophy is the most common form of storing lipids, whereas hyperplasia predominates in the prepubertal age. However, recently it has been shown that there is also a continuous turnover of adipocytes in adult men of $10 \%$ per year (51). Hypertrophic obesity is more strongly associated with insulin resistance and its metabolic complications than hyperplastic obesity $(52,53)$. A change from hypertrophic to hyperplastic obesity should ameliorate these metabolic complications. This is indeed true, since subjects treated with thiazolidindiones showed an enhanced adipose tissue growth but with an increased number of small adipocytes and a decrease in large adipocytes. Although adipose tissue mass increased, insulin resistance as well as the accompanying dyslipidemia were reduced (54). Individuals with hypertrophic obesity have been shown to possess a low degree of adipocyte differentiation and the degree of impairment was positively correlated with adipose cell size (55). Interestingly, this did not appear to be a consequence of a reduced number of precursor cells. This finding is intriguing and suggests that excess lipids cannot be stored adequately in the subcutaneous adipose tissue in hypertrophic obesity and will be directed to non-adipose organs like liver and skeletal muscle. 


\section{Disturbed adipokine expression and secretion}

Adipose tissue is an active secretory organ whose secretion profile is dramatically changed in overweight and obesity. Plasma adipocytokine levels rise with an increase in adipose tissue and adipocyte volume, except for plasma adiponectin, which is lower in obesity (56). Through such secreted products, adipocytes possess the capacity to influence local adipocyte biology, as well as systemic metabolism. Leptin and adiponectin are considered to be primary adipocytokines because they appear to be produced primarily by adipocytes. Leptin is primarily produced by white adipose tissue and acts at the brain to maintain body weight homeostasis by suppressing food intake and increasing thermogenesis via sympathetic nerve activity (57). In tissues such as muscle, leptin has been shown to stimulate lipid oxidation and reduce lipogenesis, which may contribute to improvements in insulin sensitivity (58-60). Despite the insulin-sensitizing effects of leptin, the obese state is characterized by hyperleptinemia, which indicates a state of central and peripheral leptin resistance $(61,62)$. Adiponectin has an antiinflammatory role, protecting against insulin resistance, T2DM, non-alcoholic fatty liver disease and atherosclerosis. Adiponectin acts centrally to promote weight loss and peripherally to enhance fatty acid oxidation and glucose uptake in muscles and decreases hepatic gluconeogenesis $(63,64)$. Decreased adiponectin concentrations may have detrimental effects on fat oxidation, since it has been demonstrated that adiponectin increases fat oxidation in rat skeletal muscle and C2C12 myocytes (65-67). Furthermore, a reduction in adiponectin concentration may decrease skeletal muscle glucose uptake $(66,68,69)$ and increase gluconeogenesis (67). Several inflammatory cytokines like tumor necrosis factor- $\alpha$ (TNF- $\alpha$ ), interleukin-6 (IL-6), monocyte chemotactic protein-1 (MCP-1), visfatin, and plasminogen activator inhibitor-1 (PAl-1) are expressed in adipocytes as well as activated macrophages and/or other immune cells (70). TNF- $\alpha$ increases lipolysis in human and 3T3-L1 adipocytes $(71,72)$. In addition, IL-6 has also been shown to stimulate lipolysis in vivo in humans (73). Therefore, these local effects on adipose tissue lipolysis may contribute to the development of insulin resistance. Furthermore, TNF- $\alpha$ may impair insulin sensitivity by triggering different key steps in the insulin signaling pathway (74).

\section{Mechanisms for adipose tissue inflammation}

Growing evidence links low-grade inflammation in adipose tissue with obesity and insulin resistance. During the progression to obesity, the adipose tissue expands and this may lead to hypoxia in certain parts of the adipose tissue because the blood supply is insufficient. Local adipose tissue hypoxia is partly responsible for dysregulated adipokine production and may contribute to adipocyte death $(75,76)$. However, there is still a lot of controversy if hypoxia is a trigger of the inflammatory 
response since it has been shown that adipose tissue oxygen tension was increased rather than decreased in the obese state despite a lower adipose tissue blood flow, which may be explained by a lower adipose tissue oxygen consumption in obesity (77). Furthermore, hypertrophic adipocytes are subjected to several other cytotoxic stressors, including endoplasmic reticulum stress and reactive oxygen species $(78,79)$. These cytotoxic stresses activate inflammatory signaling cascades, which leads to chemokine release and subsequent recruitment of macrophages into the adipose tissue, where they can form ring-like structures around large, dying adipocytes (80). The primary purpose of these recruited macrophages is the removal of cell debris and tissue remodeling. The precise mechanisms that regulate the recruitment of new macrophages to adipose tissue are unclear but may involve MCP-1, which is a chemokine that promotes the recruitment of monocytes to damaged or inflamed tissues, and its receptor chemokine (C-C motif) receptor 2 (CCR2) (81). Macrophage phenotypes exist across an $\mathrm{M} 1$ to $\mathrm{M} 2$ polarization state in which $\mathrm{M} 1$ cells are operationally defined as "classically activated", pro-inflammatory macrophages, while M2 cells make up the "alternatively activated", anti-inflammatory macrophage population (82). In obesity, the adipose tissue consists mainly of macrophages of the M1 phenotype (83). Accordingly, because of their pro-inflammatory properties, these macrophages are implicated in the pathogenesis of insulin resistance (84). In humans this classification in M1/M2 may be less straightforward and oversimplified but it provides a useful initial structure for distinguishing macrophage functions. Adipose tissue macrophages are surrounded by a lipid-rich environment, which can further induce macrophage inflammatory processes via activation of the pattern recognition receptors TLR2 and TLR4 $(83,85)$. In addition to macrophages, adipose tissue is known to contain other immune cells like CD4+ and CD8+ T cells, natural killer T cells, B cells, eosinophils, neutrophils, and mast cells, which may also contribute to adipose tissue inflammation (86-88).

In summary during long-term excessive energy intake, adipose tissue mass increases, to store the overload of lipids. Hypertrophic obesity, which develops, is characterized by a low degree of adipocyte differentiation and may further impair increased fatty acid storage contributing to a limited buffering capacity of the adipose tissue. This may increase the supply of lipids to non-adipose tissues like skeletal muscle and result in ectopic lipid accumulation, which is associated with the development of insulin resistance. Furthermore, expansion of the adipose tissue is associated with an altered production of adipokines and an increased inflammatory response. This may also contribute to the insulin resistant state via modulation of skeletal muscle fat metabolism or interference with the insulin signaling cascade. Besides the increased supply of lipids to skeletal muscle, also intrinsic disturbances in skeletal muscle fatty acid handling may play an important 
role in the development of insulin resistance, which will be described in more detail in the next paragraphs.

\section{SKELETAL MUSCLE FUNCTION AND DYSFUNCTION}

Skeletal muscle is responsible for $70-80 \%$ of insulin-stimulated whole-body glucose disposal and plays an important role in the pathogenesis of insulin resistance (6). Various methods have been used to investigate in vivo fatty acid metabolism in order to more clearly define the aspects of lipid metabolism that contribute to the pathogenesis of insulin resistance. Isotope dilution and indirect calorimetry techniques provide information on rates of whole-body lipid utilization $(89,90)$. Regional lipid metabolism has been studied by following changes in the interstitial glycerol concentration, using semi-permeable microdialysis catheters as well as arterio-venous $(\mathrm{A}-\mathrm{V})$ balance techniques, based upon measurement of metabolite concentrations of arterial blood and venous blood draining the organ of interest in combination with tissue blood flow determination (91-93). Disturbances in fatty acid metabolism have been suggested to contribute to the development of skeletal muscle insulin resistance. Moreover, increased muscle fat accumulation has been linked to insulin resistance and may be a consequence of an increased supply of lipids to skeletal muscle as has previously been described, or intrinsic disturbances in the regulation of fatty acid uptake, storage and/or oxidation. The next paragraphs will describe skeletal muscle fat metabolism with respect to fatty acid uptake and fatty acid partitioning towards either storage or oxidation and how disturbances in these processes may contribute to the development of the insulin resistant state. Moreover, an overview will be given of the different paradigms, which link the intrinsic disturbances in skeletal muscle fat metabolism to insulin resistance.

\section{Fatty acid uptake in skeletal muscle}

Regardless of the debate whether plasma FFA and/or TAG concentrations are increased in obesity and insulin resistant conditions or not, differences in the ability to take up fatty acids by skeletal muscle have been reported as well. For many years, it was believed that the transport of fatty acids across the plasma membrane in skeletal muscle occurred only via passive diffusion. However in recent years, many studies have demonstrated that fatty acid uptake into skeletal muscle also occurs via a protein-mediated process (94-96). The membrane-associated proteins in skeletal muscle have been identified as fatty acid translocase (FAT/CD36) (97), fatty acid binding protein (FABPpm) (98) and fatty acid transport protein (FATP1-6) $(99,100)$. Inside the cell, fatty acids remain water insoluble and are bound to one of two proteins: FABPc or acyl-CoA binding protein. FABPC is abundantly present in the cytosol and likely works together with FAT/CD36 to 
transport long chain fatty acids across the plasma membrane (101). The exact contribution of these transporters to fatty acid uptake is not clear, but FAT/CD36 is thought to be the predominant transporter, and is the one most studied (102). In addition, these transporters may interact with each other to facilitate fatty acid uptake, as interactions between FAT/CD36 and FABPpm, and between FAT/CD36 and FATP have been identified in controlling fatty acid uptake $(103,104)$. Thus, uptake of fatty acids into the skeletal muscle depends on both fatty acid concentration in the blood and tissue (blood-tissue concentration gradient) and on the regulation of the transporters (105).

Increased rates of fatty acid uptake across plasma membranes in vitro have been observed in skeletal muscle of obese individuals and T2DM subjects. The increased rates of fatty acid transport have been associated with increases in plasma membrane FAT/CD36, while FAT/CD36 protein expression was not altered, suggesting that a permanent redistribution of FAT/CD36 to the plasma membrane occurred (106). Several in vivo human studies showed no effect on skeletal muscle fatty acid uptake during fasting or postprandial conditions in obese individuals, insulin resistant subjects or subjects with impaired glucose metabolism compared with their controls $(44,107-109)$. Notably, in all of these studies circulating FFA concentrations were comparable between groups or slightly elevated in the more insulin resistant group. Two other studies reported a slightly elevated muscle FFA uptake during fasting conditions despite comparable FFA concentrations in obese and insulin resistant conditions $(18,110)$. Thus, there is not much evidence available from in vivo human studies that skeletal muscle FFA uptake is increased in the insulin resistant state.

The contribution of TAG-derived FA to skeletal muscle FA uptake is currently not fully understood but may be equally important as circulating FA $(111,112)$. The activity of LPL may be an important contributor to an increased muscle TAG extraction. Insulin has shown to stimulate LPL activity in adipose tissue but does not appear to activate LPL in skeletal muscle $(113,114)$. Downregulation of LPL increased glucose uptake in L6 muscle cells, while a tissue-specific overexpression of LPL in skeletal muscle increased cellular stores of TAG and led to insulin resistance $(115,116)$. Moreover, in insulin resistant subjects it has been shown that TAG extraction across the muscle was increased $(44,109)$. Therefore, it can be postulated that in insulin resistant conditions, the insulin mediated suppression of muscle LPL is impaired, resulting in an increased fatty acid supply to the muscle. This may possibly enhance muscle lipid accumulation, which can interfere with the insulin signaling cascade. 


\section{Fatty acid partitioning within skeletal muscle}

Once fatty acids are transported into the cytosol, fatty acids are esterified to fatty acyl-CoA by the enzyme acyl-CoA synthetase, which then enters the mitochondria for oxidation or is partitioned towards the synthesis of intramyocellular lipid (IMCL) (117).

\section{Fat storage}

The synthesis of TAG from fatty acids starts with the sequential addition of activated fatty acyl-CoAs to a glycerol backbone. The first committed step in TAG synthesis is catalyzed by glycerol-3-phosphate acyltransferase (GPAT), which results in the synthesis of lysophosphatidic acid (LPA). In subsequent steps, LPA is converted to phosphatidic acid (PA), and PA is converted to DAG. The final step in triglyceride synthesis is the addition of a fatty acyl-CoA to DAG by DAG acyltransferase (DGAT) (118). This enzyme has dual significance in that it promotes triglyceride storage but also decreases the TAG precursor DAG, which may have an inhibitory effect on insulin signaling $(119,120)$. However, unilateral DGAT overexpression in skeletal muscle has been shown to increase the storage of IMCL but remarkably also resulted in an increased DAG content even though an improvement in insulin sensitivity was observed (121). This may suggest that not DAG and TAG content per se are related to insulin resistance. Increasing DAG and TAG turnover and thus a proper balance between supply and oxidation may prevent insulin resistance. Lipid turnover is not only determined by TAG synthesis but also by TAG lipolysis. Disturbances in the regulation of skeletal muscle lipolysis may also contribute to the accumulation of lipids and lipid intermediates. Under fasting conditions it has been shown that total glycerol release was reduced across the forearm muscle of obese subjects compared with lean controls (110). Furthermore, it was shown that HSL protein content was lower and ATGL protein higher in obese insulin resistant subjects. This difference in muscle lipase content was accompanied by a lower DAG hydrolase activity and normal TAG hydrolase activity suggesting incomplete TAG hydrolysis. Surprisingly, no accumulation of DAG was observed in skeletal muscle (122). This could be partially attributed by a rapid conversion of DAG back to TAG by DGAT, which may be a protection mechanism to prevent accumulation of potential lipotoxic DAG. There is still a lot of controversy about the lipotoxic potential of several lipid metabolites. It may be that not TAG or lipid metabolite content per se is associated with insulin resistance but that muscle lipid turnover and lipid metabolite composition, and localization may play an important role. 


\section{Fat oxidation}

The capacity of the body or cells to rapidly switch substrate oxidation from predominantly lipid oxidation and high rates of fatty acid uptake during fasting conditions to the suppression of lipid oxidation and increased glucose uptake, oxidation and storage under insulin-stimulated conditions is defined as metabolic flexibility $(123,124)$. In healthy, metabolically normal individuals substrate switching occurs rapidly and completely. If skeletal muscle cannot match fat oxidation to lipid supply, fat accumulation will ensue, which will in turn cause insulin resistance. Fatty acids are the main readily available energy sources during the transition from the fed to the overnight-fasted condition. Several studies suggest that fasting respiratory quotient $(R Q)$ is elevated in skeletal muscle from obese insulin resistant and type 2 diabetic adults, which indicates lower rates of fat oxidation $(107,125)$. A reduced fat oxidation in T2DM subjects has also been reported during exercise (126) and in obese subjects after beta-adrenergic stimulation (127). Impairments in postprandial fat oxidation possibly play a larger role in fatty acid-induced insulin resistance, since most individuals are in a postprandial state for the greatest part of the day. During insulin stimulation, several studies described an impaired capacity to increase muscle and whole body glucose oxidation and a blunted suppression of fat oxidation in insulin resistant subjects $(107,123)$. After a meal, fuel switching was also disturbed in prediabetic and T2DM subjects $(108,125)$. The underlying mechanisms responsible for the impaired fat oxidation are discussed in the next paragraphs.

\section{FA uptake in mitochondria}

The rate-limiting step for $\beta$-oxidation of long-chain fatty acids is their transport into the mitochondria via carnitine palmitoyltransferase-I (CPT-1) (128). While short and medium chain fatty acyl-CoA are believed to diffuse into the mitochondria, long chain fatty acyl-CoA (LCFA-COA) require modification before crossing the inner mitochondrial membrane for oxidation. The LCFA-CoA is transported across the inner mitochondrial membrane as an acylcarnitine complex, which is synthesized via CPT-1. Once across the inner mitochondrial membrane, the acylcarnitine is converted back to the original fatty acyl-CoA and released into the matrix to enter the $\beta$-oxidation pathway (129). Two regulatory mechanisms of CPT-1 have been proposed. One involves malonyl-CoA and the other involves the cytosolic concentration of carnitine. Malonyl-CoA is an allosteric inhibitor of CPT-1, so any changes in malonyl CoA concentration can alter mitochondrial uptake of fatty acids and consequently the rate of fatty acid oxidation in the mitochondria $(130,131)$. The concentration of malonyl CoA is controlled by its turnover, in which acetyl CoA carboxylase (ACC) catalyzes the synthesis pathway, and malonyl CoA decarboxylase (MCD) controls its degradation (132). In human skeletal muscle it 
has been shown that hyperinsulinaemia increases malonyl-CoA, inhibits functional CPT-1 activity and shunts fatty acids away from oxidation towards storage (133). A key determinant of ACC activity is the activity of AMP-activated protein kinase (AMPK). This is generally called the 'fuel sensor' of the cell that is activated with an increased AMP:ATP ratio (134). AMPK can phosphorylate and inactivate ACC, which relieves CPT-1 from malonyl CoA's inhibitory effect, resulting in a stimulation of fatty acid $\beta$-oxidation (135). In skeletal muscle, it has also been suggested that AMPK can directly activate MCD (136). Recently, it has been suggested that the mitochondrial transport of fatty acyl-CoA may involve other proteins in addition to CPT-1 (137). It appears that FAT/CD36 works in conjunction with CPT-1, because they together influence rates of mitochondrial FA oxidation, and are co-localized in the mitochondria $(138,139)$. FABPpm is another well-recognized plasma membrane FA transport protein that has also been found on mitochondria (140, 141). However, the role of FABPpm with respect to mitochondrial FA oxidation remains unknown.

\section{Mitochondrial function/dysfunction}

The mitochondria in obese and insulin resistant individuals have also been hypothesized to be dysfunctional, this could be due to a decreased number of normally functioning mitochondria or a defect in individual mitochondria per se $(142,143)$. Results on the role of mitochondria in insulin resistant skeletal muscle have been conflicting. Several studies reported a reduction in mitochondrial content or reduced oxidative capacity of individual mitochondria in skeletal muscle (144-147). In other studies, however, no alterations in mitochondrial oxidative capacity were observed $(148,149)$. Some but not all studies showed reduced expression of peroxisome proliferator activated receptor $y$ coactivator $1 \alpha(P G C 1 \alpha)$ and PGC1 $\beta$, the main regulators of mitochondrial biogenesis and fatty acid oxidation in patients with T2DM and their nondiabetic relatives (150-152). The PGC1 family of coactivators responds to environmental and nutritional stimuli and is controlled at the post-transcriptional level; normal protein or mRNA levels of PGC1 $\alpha$, therefore, do not exclude impairments in protein activity or binding to docking sites (153). It still remains to be determined whether this mitochondrial dysfunction is a direct cause of insulin resistance in obesity or is a secondary consequence of obesity/insulin resistance and/or reduced physical activity. The latter may also exacerbate the metabolic disturbances associated with insulin resistance. 
Recently, an alternative hypothesis connecting fatty acid oxidation to insulin resistance has been proposed. Insulin resistance in skeletal muscle may be accompanied by a dysbalance between $\beta$-oxidation and tricarboxylic acid (TCA) cycle activity. When $\beta$-oxidation exceeds the ability of the mitochondria to utilize acetyl-CoA in the TCA cycle, there is an accumulation of incompletely oxidized, fatty acid products (i.e. acylcarnitines), which can impair skeletal muscle insulin sensitivity (154). Accordingly, patients with T2DM and obesity accumulate plasma acylcarnitines that arise from increased rates of inefficient fatty acid oxidation (155).

\section{Paradigms relating disturbances in fat metabolism to insulin resistance}

From previous paragraphs it has become clear that disturbances in skeletal muscle fatty acid metabolism may play an important role in the development of insulin resistance. Two paradigms will be discussed to explain how fatty acids can impair glucose uptake and insulin signaling.

\section{Glucose FA cycle}

In the 1960s, Randle and colleagues (156) proposed a mechanism by which fatty acids could impair insulin-stimulated glucose oxidation in muscle. This mechanism was called the glucose-fatty acid cycle. High levels of fat oxidation increased the ratios of acetyl coenzyme $A$ to coenzyme $A$ and NADH to NAD+ in the mitochondria, with subsequent inactivation of pyruvate dehydrogenase. This in turn causes citrate concentrations to increase, leading to inhibition of phosphofructokinase. Subsequent increases in intracellular glucose-6-phosphate may inhibit hexokinase activity, resulting in a reduced glucose uptake and oxidation. Several studies argue against the mechanism postulated by Randle et al., showing that the reduced insulin-stimulated glucose uptake and glycogen synthesis in type 2 diabetic patients were associated with a reduction rather than an increase in concentrations of glucose-6-phosphate in muscle $(157,158)$. Similarly, insulin stimulated concentrations of glucose-6-phosphate and rates of muscle glycogen synthesis were reduced in lean, normoglycemic, insulin-resistant first-degree relatives of patients with T2DM (159). Other findings have led to the proposal of an alternative mechanism whereby increased intramuscular lipids lead to downregulation of insulin signaling in human skeletal muscle (160). In healthy individuals, the onset of skeletal muscle insulin resistance was delayed by 3-4 hours after exposure to high concentrations of plasma fatty acids and was accompanied by increased IMCL (161). Additionally, exposure to high concentrations of plasma FFA caused insulin resistance associated with an 
induced defect in either glucose transport or phosphorylation activity, not an impairment in glycolysis (162). However, as mentioned previously, FFA concentrations are not necessarily increased in the insulin resistant state.

\section{Ectopic fat accumulation}

Accumulation of IMCL in obese skeletal muscle may result from a dysbalance between muscle fatty acid uptake and oxidation. Despite studies reporting a link between IMCL and insulin resistance, it is now evident that this is not a simple cause and effect relationship $(163,164)$. For example, endurance athletes are extremely insulin sensitive, yet have elevated IMCL levels (165). This observation, often referred to as the athlete's paradox, has led to the insight that IMCL per se does not lead to insulin resistance.

One hypothesis to explain this paradox is that trained athletes have a higher muscle oxidative capacity, while increased IMCL levels in obese subjects have been associated with a decreased oxidative capacity (165). A proper balance between supply and oxidation may prevent the development of insulin resistance. Additionally, as long as fatty acids are efficiently directed towards storage as TAG, there is no interference with insulin signaling. Indeed, it has been shown that the triglyceride synthesis enzyme DGAT increased in muscle cells in response to exercise in parallel to an increase in insulin sensitivity (166). From this paradox, it became evident that not IMCL per se but rather other lipid metabolites like DAG, ceramide and fatty acyl-CoA may interfere with the insulin signaling pathway and may contribute to the development of insulin resistance.

\section{Diacylglycerol}

DAG is an important second messenger involved in intracellular signaling and it is thought that DAG may have detrimental effects on insulin action through its ability to activate PKC $\theta$ and PKC $\varepsilon$ in muscle $(167,168)$. Inappropriate activation of PKCs may interfere with insulin action by promoting serine/threonine phosphorylation of the insulin receptor and/or IRS-1, thus preventing tyrosine phosphorylation of these proteins that are necessary for the function of the insulin signaling cascade and stimulation of glucose transport (169). In human studies, accumulation of DAG in skeletal muscle of obese, and diabetic individuals was positively correlated with the increased activity of $\operatorname{PKC} \theta(170,171)$. Although lipid infusion in humans induces insulin resistance together with increased intramyocellular DAG levels (172), the support for a role of skeletal muscle DAG accumulation in the development of type 2 diabetes is inconsistent. Recent evidence proposes that it is not total DAG content per se but rather specific DAG-subspecies that may be important. Several DAG-subspecies exist that differ in fatty acid composition and the position of the 
fatty acid on the glycerol backbone. Only 1,2-DAG accumulates in membranes and is capable of activating PKCs, while 2,3-DAG and 1,3-DAG arises from TAG hydrolysis at the lipid droplet (173-175). Several studies investigated the fatty acid composition of intramyocellular DAG and found that saturated DAG had a negative influence on insulin signaling (176). Van Hees et al. (44) reported similar total DAG and TAG levels between insulin sensitive and insulin resistant men, but a higher degree of saturation of skeletal muscle DAG in the latter group. In addition to composition, the insulin-desensitizing effects of DAG may be governed by its subcellular location (cell membrane, ER and lipid droplet) (177). The majority of the studies published in humans report total skeletal muscle DAG concentration, without measuring compartmentalization within the cell. Since the adverse effects of PKC are confined to cell membranes, it is likely that only DAGs located in certain compartments influence insulin signaling. Bergman et al. (178) were the first to reveal that membrane localization of DAG in skeletal muscle, rather than total intramuscular concentration, drives insulin resistance in humans and that especially saturated DAG had a particularly negative impact on insulin sensitivity.

\section{Ceramides}

Another group of lipid intermediates whose contribution in the development of insulin resistance has been proposed are the ceramides, which belong to the sphingolipids. Ceramides can accumulate in skeletal muscle either by the hydrolysis of sphingomyelin (a phospholipid located in the lipid bilayer of the cell) (179), or by de novo synthesis from long-chain saturated fatty acids (180). Ceramides are important membrane building blocks and intracellular messengers that are believed to interfere with insulin signaling. It has been postulated that ceramides inhibit insulin action via the inhibition of Akt phosphorylation, resulting in an inhibition of GLUT4 translocation $(181,182)$.

\section{Fatty acyl CoA}

Lipid intermediates that have received less attention so far are fatty acyl-CoAs. If fatty acid uptake exceeds the capacity for directing the fatty acids towards lipid synthesis or $\beta$-oxidation, fatty acyl-CoA will accumulate in the cell. Fatty acyl-CoAs have been proposed as a better predictor of insulin resistance than IMCL (183). It remains unclear through which mechanism(s) fatty acyl-CoAs may affect insulin action, but some data suggests that fatty acyl-CoAs may interfere with insulin signaling through the activation of PKC isoforms (169). It is also possible that fatty acyl-CoAs act indirectly by acting as a precursor of other lipid intermediates such as DAG and ceramide. 


\section{CONCLUDING REMARKS}

It is clear that the development of insulin resistance is associated with disturbances in lipid metabolism. Despite intense research, current understanding of mechanisms underlying lipid-induced insulin resistance remains incomplete. The majority of evidence supports the concept that a dysbalance between fatty acid uptake and oxidation may lead to ectopic lipid accumulation, which can interfere with insulin signaling. It seems that not lipid content per se is associated with insulin resistance but that rather muscle lipid turnover and lipid metabolite composition and localization play an important role. Furthermore, the dysbalance may be explained by an increased supply of lipids from the circulation or an intrinsic disturbance in fatty acid uptake, storage and/or oxidation. It seems that elevated TAG concentrations may have a greater contribution to the increased supply of lipids to skeletal muscle than FFA. These elevated TAG concentrations may arise from a limited buffer capacity of the adipose tissue or an increased liver VLDL production. Further studies are needed to improve the knowledge of mechanisms contributing to lipid induced insulin resistance.

\section{FAT METABOLISM IN RELATION TO BODY WEIGHT CONTROL}

Body composition and body mass can remain stable over time only if the metabolizable energy ingested matches the energy being expended, so if the individual remains in energy balance. For weight maintenance, not only energy balance plays an important role, but also macronutrient intake must balance macronutrient oxidation. This means that over time, the quantity of carbohydrate, fat and protein consumed equals the amount of each oxidized (184). Protein intake is usually around $15 \%$ of dietary energy. The daily protein intake is about $1 \%$ of the total protein stores (185). The protein stores increase in size only in response to growth stimuli, such as growth hormone, androgens, physical training and weight gain, but not simply from increased dietary protein. Thus, protein stores are tightly controlled and protein balance is achieved on a day-to-day basis. Carbohydrate is usually the main source of dietary energy, yet the body stores of glycogen are very limited to amounts of about $500-1000 \mathrm{~g}$ (186). Daily intake of carbohydrate corresponds to about $50-100 \%$ of the carbohydrate stores compared with about $1 \%$ for protein and fat (187). Excess carbohydrate intake cannot be the basis of weight gain because the storage capacity is limited and controlled, and conversion to fat only occurs under extreme conditions in humans (186). In contrast to the other nutrients, body fat stores are large, and fat intake has no or very little influence on fat oxidation (187). In response to an increase in dietary fat content, fat oxidation can take more than 1 week to match fat intake (188). Any excess of 
fat in the everyday diet is stored and contributes to an increased adipose tissue mass $(189,190)$. However, there are individual differences in the extent to which a high dietary fat intake results in increased fat oxidation and body fat gain. There are indications that obese subjects may have a diminished capacity to oxidize fat and adapt more slowly to a high dietary fat intake compared with lean subjects $(189,191)$. A limited capacity to adjust fat oxidation to fat intake may translate into a positive fat balance and weight gain in the long term (192).

Flatt postulated a two-compartment model in which it is stated that when the dietary fat content increases, fat oxidation can be raised by either maintenance of low glycogen stores or by expansion of the adipose tissue mass (193). Indeed, it has been shown that obese subjects are capable of rapidly adjusting fat oxidation to fat intake when glycogen stores are lowered by an exhaustive exercise test (194). Also, ad libitum consumption of a high fat diet has been shown to expand body fat stores, which increased fat oxidation until a new equilibrium is reached between fat intake and fat oxidation. A 10-kg change in fat mass has been shown to correspond to a change in fat oxidation of $11 \mathrm{~g} / \mathrm{d}(8-14 \mathrm{~g} / \mathrm{d})(195)$. Weight maintenance requires that the body's metabolism oxidizes as much glucose, fatty acids, and amino acids as are supplied by the diet. The goal is to achieve this steady state without the need for an undesirable expansion of the adipose tissue mass. Therefore, dietary interventions that may promote fat oxidation are currently an area of intense investigation since they may be beneficial for both body weight control as well as reducing ectopic fat accumulation promoting thereby insulin sensitivity.

\section{DIETARY INTERVENTIONS FOR IMPROVING INSULIN SENSITIVITY AND BODYWEIGHT CONTROL}

Changes in lifestyle are considered to play an important role in the etiology of obesity and T2DM. Manipulation of diet and physical activity are the first-choice treatment for these metabolic diseases. Sustaining a healthy dietary lifestyle to facilitate weight loss is often difficult in an environment where palatable high-fat foods are easily available. Dietary approaches to increase the effectiveness of dietary intervention are being studied. The identification of food and food ingredients that could influence specific physiological targets like increasing fat oxidation and restoring metabolic flexibility may be helpful in the prevention and treatment of obesity as well as diabetes (196). 


\section{CALORIE RESTRICTION}

Calorie restriction has consistently been proven to produce weight loss and to have beneficial health effects like an improved insulin sensitivity $(197,198)$, possibly mediated via modulation of fat metabolism. Weight loss showed either an improvement (199) or no change (107) in fasting fat oxidation. After weight loss, obese prediabetic subjects have been shown to improve their capacity to switch from fat oxidation to carbohydrate oxidation after a meal (108). Altogether, there are clear indications that weight loss is able to partly reverse postprandial impairments in substrate oxidation, which may be positively associated with insulin sensitivity, whereas with respect to the fasting condition, improved regulation of fat oxidation could not be confirmed in all studies. In post-obese women an impaired postprandial fat oxidation may contribute to weight regain (200). Furthermore, weight loss induced by calorie restriction has been shown to improve insulin sensitivity and to reduce IMCL in obese subjects $(201,202)$. The reduction in IMCL may be due to an increased lipid oxidation capacity. At the molecular level in skeletal muscle, the expression of genes involved in the regulation of mitochondrial biogenesis was increased after calorie restriction. In parallel, mitochondrial content increased in the calorie restriction group (203). The molecular mechanisms that underlie the upregulation of mitochondrial mass and function may be related to Sirtuin-1 (SIRT1) which deacytylates and activates PGC1 $\alpha$ suggesting a direct link between SIRT1 and mitochondrial bioenergetics (204, 205). Surgically-induced weight loss increased PGC1 $\alpha$ expression in previous morbidly obese subjects $(206,207)$, while Berggren et al. (208) observed no change in PGC1 $\alpha$ expression after weight loss in obese subjects.

Underlying mechanisms mediating the effects of calorie restriction on insulin sensitivity may involve changes in skeletal muscle fatty acid handling but this needs to be further elucidated.

\section{RESVERATROL}

Polyphenolic compounds, such as resveratrol have, recently, received wide interest due to their ability to mimic effects of calorie restriction. Resveratrol is found in peanuts, grapes and red wine, with potential therapeutic implications including antiinflammatory, antioxidant, cardio-protective, anti-aging actions and beneficial properties against metabolic diseases (209). Although originally the focus was mainly directed towards anti-oxidant properties of resveratrol (210), more recently cellular effects on fatty acid metabolism and mitochondrial function have been described. By reversing disturbances in fat metabolism resveratrol may be able to improve the success of lifestyle interventions with respect to insulin sensitivity and body weight control. 


\section{Glucose metabolism and insulin sensitivity}

Insulin resistance has been linked to IMCL accumulation which may develop because of an impaired mitochondrial function that directs fatty acids towards storage, as opposed to oxidation $(211,212)$. In line with this, resveratrol has shown to improve both muscle oxidative capacity and insulin sensitivity in high fat-fed mice (213). The effect of resveratrol on the induction of oxidative phosphorylation genes and mitochondrial biogenesis was largely explained by the activation of SIRT1, which decreased PGC1 $\alpha$ acetylation and increased PGC1 $\alpha$ activity.

Besides the ability of resveratrol to activate SIRT1, recent work has shown that many of the favourable effects of polyphenols occur in an AMPK-dependent manner, indicating that AMPK signaling is likely involved in the insulin-sensitizing effects of these compounds (214). Auwerx and colleagues (215) proposed that AMPK might be an upstream target of resveratrol or calorie restriction prior to SIRT1 activation. Some studies have shown that resveratrol can stimulate glucose uptake independent of the action of insulin as well as enhance insulin dependent glucose transport (216-218). In the absence of insulin, the effect of resveratrol on glucose uptake was primarily dependent on AMPK activation, without involving PI3K. In the presence of insulin, resveratrol also potentiated the effect of insulin on glucose uptake via AMPK activation, but leading to activation of the PI3K-Akt signal pathway (218). In T2DM patients it was found that low dosages of resveratrol decreased blood glucose levels and improved insulin sensitivity, as measured by the HOMA index (219).

\section{Body weight control}

Resveratrol has been shown to markedly attenuate weight gain in association with induction of mitochondrial biogenesis in skeletal muscle and brown adipose tissue in high-fat fed mice. In parallel, the resveratrol-treated mice showed increased endurance capacity, higher oxygen consumption, and improved glucose tolerance (213). In contrast, Baur et al. (220) reported that although resveratrol supplementation was beneficial in protecting high-fat fed mice against some of the negative health consequences of obesity, such as insulin resistance, the supplementation did not protect them from weight gain. The reason for the discrepancy is not clear, however, it may be due to the dose of the resveratrol used and/or to the length of the study. Further research is necessary to investigate the effect of resveratrol on body weight regulation.

\section{Resveratrol and adipose tissue function}

The effect of resveratrol on adipose tissue metabolism is mediated, at least in part, by a reduction in fatty acid uptake from TAG in circulating lipoproteins caused by a 
reduced LPL activity (221). The effects of resveratrol on lipolysis have been analysed by Szkudelska et al. (222). They observed an enhanced lipolytic response to epinephrine induced by resveratrol. No changes were observed in mRNA levels of ATGL, while HSL mRNA expression was increased in resveratroltreated animals, showing that this molecule acts differently on both lipases (222). In contrast Lasa et al. (223) demonstrated an increased ATGL gene and protein expression, while HSL expression was not changed. Resveratrol has been shown to promote fat mobilization through activation of SIRT1 and suppression of peroxisome proliferator-activated receptor- $\gamma$ (PPAR $\gamma$ ), resulting in decreased TAG storage and increased secretion of FFA. Furthermore, resveratrol inhibited insulinstimulated de novo lipogenesis. This effect was accompanied by the downregulation of key lipogenic molecules, such as Fatty acid synthase (FAS) and ACC (224). Overall, resveratrol has been proven to promote lipolysis and inhibit lipogenesis in murine animals and in vitro experiments. This may lead to a diminished lipid accumulation in adipocytes and a decrease in adiposity. Nevertheless, this may increase the supply of FFA and TAG to non-adipose organs such as liver and skeletal muscle and may possibly contribute to impairments in insulin sensitivity if fat oxidation is not matched. Therefore, further research is necessary in order to determine resveratrol's whole body mechanism of action and how it affects the balance between adipose tissue lipolysis and muscle fat oxidation. Resveratrol has also shown to reduce proinflammatory cytokine expression in cultured adipocytes $(225,226)$ and in rodent white adipose tissue $(227)$. Because adipose tissue inflammation is tightly linked to systemic insulin resistance, resveratrol-induced down-regulation of the adipose expression of cytokines such as II-6, MCP-1, TNF $\alpha$ and PAl-1 may contribute to the insulin sensitizing effect of resveratrol supplementation $(213,220,225-228)$. In addition, resveratrol has shown to up-regulate the expression of adiponectin - an adipokine with wellestablished antidiabetic and anti-inflammatory action - both in cultured adipocytes and in WAT of obese Zucker rats $(227,229)$. In general, it has been established that resveratrol holds anti-inflammatory properties.

In summary, resveratrol represents the first in a novel class of SIRT1 activators that are proven to be safe and well tolerated in humans. It might be a good candidate for drug development because it mimics the metabolic benefits of calorie restriction like improving mitochondrial function, insulin sensitivity and modulation of adipose tissue function but human data are still lacking (230). 


\section{DIETARY FATTY ACIDS}

In most western countries, energy intake from fat is around $35-40 \%$ of total energy. Through our diet we are exposed to a variety of different fatty acids like saturated fatty acids (SFA), monounsaturated fatty acids (MUFA) and polyunsaturated fatty acids (PUFA), which are reflected in lipids of plasma and tissues. An overconsumption of saturated fats has been implicated as a causative factor for the development of insulin resistance, whereas certain monounsaturated fats and PUFAs and, particularly n-6 fatty acids, appear to have no adverse effects or even fairly positive effects on the action of insulin (231). Epidemiological evidence suggests that insulin resistance is linked to the ingestion of saturated fats (232234). Therefore, it is important to understand the underlying mechanisms how dietary fatty acids may affect insulin sensitivity in skeletal muscle and adipose tissue and how the risk or severity of insulin resistance can be modulated by dietary fat quantity and quality.

\section{Glucose metabolism and insulin sensitivity: evidence from human dietary interventions}

In the KANWU study by Vessby et al. (235), healthy subjects were randomly assigned to either a diet rich in SFA or a diet with the same amount of calories from MUFA. A random subsample in each group was also given a supplement of $n-3$ fatty acids. Decreasing SFA and increasing MUFA, improved insulin sensitivity. However, a beneficial impact of the fat quality on insulin sensitivity was not seen in individuals with a high habitual fat intake ( $>37 \mathrm{E} \%$ ). The addition of $n-3$ fatty acids did not result in any change in insulin sensitivity (235). These data suggest that dietary fat quality is only of importance for insulin sensitivity with a total fat intake equal or lower than $35-40 \%$ of total energy intake. Summers et al. demonstrated in a randomized controlled crossover study that a n-6 PUFA-rich diet improved insulin sensitivity when substituted for a SFA-rich diet, after only 5 weeks (236). However, in the LIPGENE study no direct effects on insulin sensitivity were reported after a 12-week dietary intervention with isoenergetic diets differing in both fat quantity and quality (237). Furthermore, in the RISCK trial, isoenergetic replacement of SFA with MUFA did not improve insulin sensitivity after a 24-week intervention in subjects at risk for the metabolic syndrome (238). The data on the effect of diets differing in fat quality on insulin resistance are inconsistent and further research is necessary. A possible explanation for the conflicting results may be that a different habitual intake of subjects may be an important determinant for the effectiveness of a dietary intervention. 


\section{Underlying mechanisms to explain the effects of dietary fatty acids}

Dietary fatty acids may influence insulin sensitivity via different mechanisms. PUFA have been shown to channel lipids towards inert TAG storage, while saturated fats preferentially generate "toxic" DAG and promote insulin resistance (239). Furthermore, oleic acid accumulates to a lesser extent as DAG or TAG than palmitic acid. Also, T2DM myotubes expressed a reduced palmitate oxidation whereas oleic acid oxidation was not different between groups (240). Overall, the order of preference for oxidation appears to be the highest for PUFA, followed by MUFA and eventually SFA. Piers et al. (241) reported in men that the postprandial fat oxidation rate is higher after a MUFA than after a SFA meal. Nevertheless, Casas et al. (242) did not observe any difference in substrate oxidation or satiety after the consumption of meals high in PUFA, MUFA or SFA. A secondary hierarchy may exist between fatty acids within the same class of saturation, for example shorter chain saturated fatty acids may be oxidized to a greater extent than longer chain fatty acids $(243,244)$.

Furthermore, different FA subtypes may alter several intracellular pathways by direct regulatory effects on gene expression. It has been demonstrated that PUFA suppress the transcription of genes encoding for lipogenic enzymes (FAS, ACC and SCD1, SREBP), while they induce the transcription of genes encoding proteins involved in lipid oxidation and fatty acid transport (245). Furthermore, in a subcohort of the LIPGENE study it was shown that a 12-week MUFA diet and a low-fat diet supplemented with n-3 PUFA reduced lipogenic gene expression, which shifted the balance from storage to oxidation in insulin resistant subjects (246).

There is now considerable evidence linking obesity and insulin resistance to the fatty acid composition of membrane phospholipids (247). The fatty acid composition of membrane lipids is influenced by the dietary fatty acid profile. Membrane composition was found to be most responsive to $n-6$ and n-3 PUFA levels in the diet. This is probably due to the fact that both $n-6$ and n-3 PUFA classes cannot be synthesized de novo by higher animals (248). A high proportion of SFAs in the muscle membrane may impair insulin receptor binding and affinity, by reduced ion permeability, by an altered ability to translocate glucose transporters or by interaction with second messengers such as DAG within the muscle cell $(249,250)$. Highly unsaturated n-3 PUFAs have been shown to increase insulin receptor function and translocation of the GLUT4 transporter (251, 252). 
Dietary fat quality may also differentially affect inflammation. Exposure of myotubes or adipocytes to SFA increased IL-6 mRNA expression and subsequent protein production, possibly via activation of NF-KB $(253,254)$. A human intervention study feeding diets rich in SFA for 5 weeks showed higher concentrations of CRP, fibrinogen and IL-6 compared with a diet enriched in MUFA (255). Substantial increase in the intake of PUFA can decrease low-grade inflammation as indicated by circulating CRP, IL-6 or soluble adhesion molecules (256).

All these data indicate that both fat quality and quantity play a role in insulin sensitivity and this should not be neglected in the search for optimal diets to improve insulin sensitivity.

\section{DIETARY FIBERS}

Fiber is a major component of carbohydrate that is generally considered beneficial for health. Current recommendations suggest an intake of 20-40 g dietary fiber per day (257). Consumption of diets high in dietary fiber have been reported to result in a number of beneficial health effects including reduced risk of heart disease (258), hypertension (259), colon cancer (260), diabetes (261) and obesity (262). Fiber sources differ in characteristics such as solubility, fermentability, and viscosity, and it is now well known that different types of fiber exert varying physiological effects in the body.

\section{Glucose metabolism and insulin sensitivity}

Fiber-rich foods usually have lower energy content, which contributes to a decrease in the energy density of the diet. In the intestine, the incorporation of fiber may complicate the union between digestive enzymes and their substrate, thus slowing down the absorption of nutrients (263). The slower nutrient uptake may result in a decrease in glucose and insulin responses (264-268). These lower glucose and insulin concentrations may, in turn result in a reduced inhibition of lipolysis, higher circulating FFA concentrations and consequently increased fat oxidation $(269,270)$. A greater postprandial fat oxidation may attenuate fat accumulation in non-adipose tissues, leading to an improvement in insulin sensitivity (271). In the Finnish Diabetes Prevention Study, individuals with the highest level of fiber consumption had a $62 \%$ reduction in progression of prediabetes to diabetes over a 4-year period compared with those with the lowest fiber intake (272). Short-term studies indicate that dietary fiber intake decreases postprandial glycemia and insulinemia and enhances insulin sensitivity $(273,274)$. Pereira et al. (275) used a euglycemic-hyperinsulinemic clamp to measure insulin sensitivity in obese hyperinsulinaemic subjects. Their results showed that a whole grain diet led to a postprandial improvement in insulin sensitivity when compared to 
a refined grain diet (275). Likewise, Weickert et al. (273) used the same method to measure insulin sensitivity in overweight and obese women and found an increase in insulin sensitivity after 3 days of a diet containing bread enriched with insoluble fiber compared to another diet containing white bread.

\section{Body weight control}

High dietary fiber intake may promote weight loss and long-term weight maintenance by reducing energy intake and appetite (276). Greater satiety may result from several factors. High fiber foods take longer to eat and may slow gastric emptying thereby increasing feelings of fullness and satiety. They may also stimulate the release of gut peptides that modify eating behaviour $(277,278)$. Fermentation products of dietary fibers like short chain fatty acids may enhance satiety via various mechanisms (279). A number of human intervention studies have shown weight reduction with diets rich in dietary fiber or dietary fiber supplements, however other studies failed to show any effect (280-282). These conflicting results in humans may be due to the dietary fiber type, dosage and study population.

Evidence of the health-protective effects of dietary fiber has led to industrial interest in developing fiber ingredients to increase the fiber content of processed foods. There is debate as to whether these functional soluble fibers deliver known health benefits associated with 'traditional' dietary fibers (283). Many fibers have been shown to have the potential for beneficial short-term functional effects but have not been tested in long-term clinical trials. 


\section{OUTLINE OF THE THESIS}

This thesis provides insight in the effects of several dietary interventions on body weight control as well as the improvement in insulin sensitivity by modulation of the fat metabolism.

The effects of calorie restriction on insulin sensitivity may be mediated by changes in skeletal muscle fatty acid handling but the underlying mechanisms need to be further elucidated. Therefore, in chapter 2 we investigated the effect of a 12-week weight loss intervention on insulin sensitivity and on skeletal muscle fatty acid metabolism-related gene expression in obese IGT subjects. The weight loss program was based on a very low-calorie diet using meal replacements, which were gradually replaced by normal meals.

Identification of foods and food supplements that influence specific physiological targets may be helpful to improve overall metabolic health. Polyphenolic compounds, such as resveratrol are currently an area of intense investigation due to their ability to mimic effects of metabolic benefits of calorie restriction but human data are still lacking. Chapter $\mathbf{3}$ describes the first human study that systematically examined the metabolic effects of resveratrol in healthy obese men. After 30 days resveratrol supplementation a combination of in vivo and ex vivo measurements were conducted to examine the effects on whole-body energy expenditure, substrate utilization, ectopic lipid storage, mitochondrial function and lipolysis in adipose tissue and skeletal muscle. Modulation of adipose tissue function may contribute to a reduced body fat accumulation and improved insulin sensitivity. However, data on the effects of resveratrol on adipose tissue function in the human in vivo situation are lacking. Therefore, in chapter 4 the effect of resveratrol on adipose tissue morphology and the underlying mechanisms involved were investigated using microarray analysis.

Another dietary intervention to prevent obesity or its metabolic complications is reducing the amount of fat in the diet. High fat diets have been proposed to lead to obesity and may affect insulin sensitivity. Dietary fat quality may modulate fat metabolism, thereby possibly improving insulin sensitivity. Therefore, in chapter 5 we investigated the acute effects of meals with various fatty acid composition on skeletal muscle fatty acid processing and postprandial insulin sensitivity in obese, insulin-resistant men using the dual-stable isotope technique. With this technique, the contribution of dietary fat (CM-TAG) vs. endogenous fat (FFA and VLDL-TAG) to in vivo skeletal muscle fatty acid metabolism could be investigated. Moreover, baseline and postprandial skeletal muscle biopsy samples were collected for assessing intracellular lipid partitioning and examining the transcriptional regulation of fatty acid metabolism by means of microarray technology. 
Besides fat content also carbohydrates contribute to a large part of the diet. Fiber is a major component of carbohydrates and has been reported to result in several health effects. High fiber intake may decrease glycaemic and insulinemic responses, which favour oxidation and lipolysis of fat rather than storage and may attenuate fat accumulation in non-adipose tissues leading to improved insulin sensitivity. A higher postprandial fat oxidation may also translate in a negative energy balance in the long term. This has led to industrial interest in developing dietary fibers that can be incorporated in a variety of food products. However, the question remains if these fibers provide the same health benefits as traditional dietary fibers. For that reason, we studied in chapter 6 replacement of available carbohydrates with polydextrose or soluble corn fiber on glycemic and insulinemic profile, energy expenditure, substrate oxidation and appetite ratings over a period of 24 hours in overweight men and women.

Finally, in chapter 7 the main findings of the different studies described in this thesis are discussed in a broader perspective and implications for future research are addressed. 


\section{REFERENCES}

1. Obesity: preventing and managing the global epidemic. Report of a WHO consultation. World Health Organ Tech Rep Ser 2000;894:i-xii, 1-253.

2. Ingelsson E, Arnlov J, Sundstrom J, Riserus U, Michaelsson K, Byberg L. Relative importance and conjoint effects of obesity and physical inactivity for the development of insulin resistance. Eur J Cardiovasc Prev Rehabil 2009;16(1):28-33.

3. Kemper HC, Stasse-Wolthuis M, Bosman W. The prevention and treatment of overweight and obesity. Summary of the advisory report by the Health Council of The Netherlands. Neth $\mathrm{J}$ Med 2004;62(1):10-7.

4. Ferrannini E, Haffner SM, Mitchell BD, Stern MP. Hyperinsulinaemia: the key feature of a cardiovascular and metabolic syndrome. Diabetologia 1991;34(6):416-22.

5. Yki-Jarvinen H, Young AA, Lamkin C, Foley JE. Kinetics of glucose disposal in whole body and across the forearm in man. J Clin Invest 1987;79(6):1713-9.

6. DeFronzo RA, Gunnarsson R, Bjorkman O, Olsson M, Wahren J. Effects of insulin on peripheral and splanchnic glucose metabolism in noninsulin-dependent (type II) diabetes mellitus. J Clin Invest 1985;76(1):149-55.

7. Santaguida PL, Balion C, Hunt D, et al. Diagnosis, prognosis, and treatment of impaired glucose tolerance and impaired fasting glucose. Evid Rep Technol Assess (Summ) 2005(128):1-11.

8. Kahn SE, Prigeon RL, McCulloch DK, et al. Quantification of the relationship between insulin sensitivity and beta-cell function in human subjects. Evidence for a hyperbolic function. Diabetes 1993;42(11):1663-72.

9. Polonsky KS, Given BD, Van Cauter E. Twenty-four-hour profiles and pulsatile patterns of insulin secretion in normal and obese subjects. J Clin Invest 1988;81(2):442-8.

10. Kahn SE. Clinical review 135: The importance of beta-cell failure in the development and progression of type 2 diabetes. J Clin Endocrinol Metab 2001;86(9):4047-58.

11. Chang L, Chiang SH, Saltiel AR. Insulin signaling and the regulation of glucose transport. Mol Med 2004;10(7-12):65-71.

12. Frosig C, Rose AJ, Treebak JT, Kiens B, Richter EA, Wojtaszewski JF. Effects of endurance exercise training on insulin signaling in human skeletal muscle: interactions at the level of phosphatidylinositol 3-kinase, Akt, and AS160. Diabetes 2007;56(8):2093-102.

13. Duncan RE, Ahmadian M, Jaworski K, Sarkadi-Nagy E, Sul HS. Regulation of lipolysis in adipocytes. Annu Rev Nutr 2007;27:79-101.

14. Ong JM, Kern PA. Effect of feeding and obesity on lipoprotein lipase activity, immunoreactive protein, and messenger RNA levels in human adipose tissue. J Clin Invest 1989;84(1):30511.

15. Wahren J, Ekberg K. Splanchnic regulation of glucose production. Annu Rev Nutr 2007;27:329-45.

16. Lambert JE, Parks EJ. Postprandial metabolism of meal triglyceride in humans. Biochim Biophys Acta 2012.

17. Cryer A, Riley SE, Williams ER, Robinson DS. Effect of nutritional status on rat adipose tissue, muscle and post-heparin plasma clearing factor lipase activities: their relationship to triglyceride fatty acid uptake by fat-cells and to plasma insulin concentrations. Clin Sci Mol Med 1976;50(3):213-21.

18. Bickerton AS, Roberts R, Fielding BA, et al. Preferential uptake of dietary Fatty acids in adipose tissue and muscle in the postprandial period. Diabetes 2007;56(1):168-76.

19. Frayn KN. Adipose tissue as a buffer for daily lipid flux. Diabetologia 2002;45(9):1201-10.

20. Vernon RG. Effects of diet on lipolysis and its regulation. Proc Nutr Soc 1992;51(3):397-408.

21. Lelliott C, Vidal-Puig AJ. Lipotoxicity, an imbalance between lipogenesis de novo and fatty acid oxidation. Int J Obes Relat Metab Disord 2004;28 Suppl 4:S22-8.

22. Sniderman AD, Bhopal R, Prabhakaran D, Sarrafzadegan N, Tchernof A. Why might South Asians be so susceptible to central obesity and its atherogenic consequences? The adipose tissue overflow hypothesis. Int J Epidemiol 2007;36(1):220-5.

23. Summers SA. Ceramides in insulin resistance and lipotoxicity. Prog Lipid Res 2006;45(1):4272.

24. Henry SL, Bensley JG, Wood-Bradley RJ, Cullen-McEwen LA, Bertram JF, Armitage JA. White adipocytes: more than just fat depots. Int J Biochem Cell Biol 2012;44(3):435-40.

25. Lafontan M, Langin D. Lipolysis and lipid mobilization in human adipose tissue. Prog Lipid Res 2009;48(5):275-97. 
26. Goldberg IJ, Eckel RH, Abumrad NA. Regulation of fatty acid uptake into tissues: lipoprotein lipase- and CD36-mediated pathways. J Lipid Res 2009;50 Suppl:S86-90.

27. Frayn KN, Coppack SW, Fielding BA, Humphreys SM. Coordinated regulation of hormonesensitive lipase and lipoprotein lipase in human adipose tissue in vivo: implications for the control of fat storage and fat mobilization. Adv Enzyme Regul 1995;35:163-78.

28. Frayn KN, Shadid S, Hamlani R, et al. Regulation of fatty acid movement in human adipose tissue in the postabsorptive-to-postprandial transition. Am J Physiol 1994;266(3 Pt 1):E30817.

29. Villena JA, Roy S, Sarkadi-Nagy E, Kim KH, Sul HS. Desnutrin, an adipocyte gene encoding a novel patatin domain-containing protein, is induced by fasting and glucocorticoids: ectopic expression of desnutrin increases triglyceride hydrolysis. J Biol Chem 2004;279(45):4706675.

30. Fredrikson G, Tornqvist $\mathrm{H}$, Belfrage P. Hormone-sensitive lipase and monoacylglycerol lipase are both required for complete degradation of adipocyte triacylglycerol. Biochim Biophys Acta 1986;876(2):288-93.

31. Ducharme NA, Bickel PE. Lipid droplets in lipogenesis and lipolysis. Endocrinology 2008;149(3):942-9.

32. Brasaemle DL, Rubin B, Harten IA, Gruia-Gray J, Kimmel AR, Londos C. Perilipin A increases triacylglycerol storage by decreasing the rate of triacylglycerol hydrolysis. J Biol Chem 2000;275(49):38486-93.

33. Lass A, Zimmermann R, Haemmerle G, et al. Adipose triglyceride lipase-mediated lipolysis of cellular fat stores is activated by CGI-58 and defective in Chanarin-Dorfman Syndrome. Cell Metab 2006;3(5):309-19.

34. Yang $X$, Lu X, Lombes $M$, et al. The $G(0) / G(1)$ switch gene 2 regulates adipose lipolysis through association with adipose triglyceride lipase. Cell Metab 2010;11(3):194-205.

35. Lonnroth $\mathrm{P}$, Smith $\mathrm{U}$. The antilipolytic effect of insulin in human adipocytes requires activation of the phosphodiesterase. Biochem Biophys Res Commun 1986;141(3):1157-61.

36. Karpe F, Dickmann JR, Frayn KN. Fatty acids, obesity, and insulin resistance: time for a reevaluation. Diabetes 2011;60(10):2441-9.

37. Campbell PJ, Carlson MG, Nurjhan N. Fat metabolism in human obesity. Am J Physiol 1994;266(4 Pt 1):E600-5.

38. Mittendorfer B, Magkos F, Fabbrini E, Mohammed BS, Klein S. Relationship between body fat mass and free fatty acid kinetics in men and women. Obesity (Silver Spring) 2009;17(10):1872-7.

39. McQuaid SE, Hodson L, Neville MJ, et al. Downregulation of adipose tissue fatty acid trafficking in obesity: a driver for ectopic fat deposition? Diabetes 2011;60(1):47-55.

40. Langin D, Dicker A, Tavernier G, et al. Adipocyte lipases and defect of lipolysis in human obesity. Diabetes 2005;54(11):3190-7.

41. Jocken JW, Langin D, Smit E, et al. Adipose triglyceride lipase and hormone-sensitive lipase protein expression is decreased in the obese insulin-resistant state. J Clin Endocrinol Metab 2007;92(6):2292-9.

42. Arner P, Bolinder J, Engfeldt P, Hellmer J, Ostman J. Influence of obesity on the antilipolytic effect of insulin in isolated human fat cells obtained before and after glucose ingestion. $J$ Clin Invest 1984;73(3):673-80.

43. Riemens SC, Sluiter WJ, Dullaart RP. Enhanced escape of non-esterified fatty acids from tissue uptake: its role in impaired insulin-induced lowering of total rate of appearance in obesity and Type II diabetes mellitus. Diabetologia 2000;43(4):416-26.

44. van Hees AM, Jans A, Hul GB, Roche HM, Saris WH, Blaak EE. Skeletal muscle fatty acid handling in insulin resistant men. Obesity (Silver Spring) 2011;19(7):1350-9.

45. Potts JL, Coppack SW, Fisher RM, Humphreys SM, Gibbons GF, Frayn KN. Impaired postprandial clearance of triacylglycerol-rich lipoproteins in adipose tissue in obese subjects. Am J Physiol 1995;268(4 Pt 1):E588-94.

46. Panarotto D, Remillard P, Bouffard L, Maheux P. Insulin resistance affects the regulation of lipoprotein lipase in the postprandial period and in an adipose tissue-specific manner. Eur $\mathrm{J}$ Clin Invest 2002;32(2):84-92.

47. Coppack SW, Evans RD, Fisher RM, et al. Adipose tissue metabolism in obesity: lipase action in vivo before and after a mixed meal. Metabolism 1992;41(3):264-72.

48. Sadur CN, Yost TJ, Eckel RH. Insulin responsiveness of adipose tissue lipoprotein lipase is delayed but preserved in obesity. J Clin Endocrinol Metab 1984;59(6):1176-82. 
49. Musliner TA, Herbert PN, Kingston MJ. Lipoprotein substrates of lipoprotein lipase and hepatic triacylglycerol lipase from human post-heparin plasma. Biochim Biophys Acta 1979;575(2):277-88.

50. Bickerton AS, Roberts R, Fielding BA, et al. Adipose tissue fatty acid metabolism in insulinresistant men. Diabetologia 2008;51(8):1466-74.

51. Spalding KL, Arner E, Westermark PO, et al. Dynamics of fat cell turnover in humans. Nature 2008;453(7196):783-7.

52. Arner P, Arner E, Hammarstedt A, Smith U. Genetic predisposition for Type 2 diabetes, but not for overweight/obesity, is associated with a restricted adipogenesis. PLoS One $2011 ; 6(4): e 18284$.

53. Kim JY, van de Wall E, Laplante $M$, et al. Obesity-associated improvements in metabolic profile through expansion of adipose tissue. J Clin Invest 2007;117(9):2621-37.

54. Akazawa S, Sun F, Ito M, Kawasaki E, Eguchi K. Efficacy of troglitazone on body fat distribution in type 2 diabetes. Diabetes Care 2000;23(8):1067-71.

55. Isakson P, Hammarstedt A, Gustafson B, Smith U. Impaired preadipocyte differentiation in human abdominal obesity: role of Wnt, tumor necrosis factor-alpha, and inflammation. Diabetes 2009;58(7):1550-7.

56. Skurk T, Alberti-Huber C, Herder C, Hauner H. Relationship between adipocyte size and adipokine expression and secretion. J Clin Endocrinol Metab 2007;92(3):1023-33.

57. Prior LJ, Eikelis N, Armitage JA, et al. Exposure to a high-fat diet alters leptin sensitivity and elevates renal sympathetic nerve activity and arterial pressure in rabbits. Hypertension 2010;55(4):862-8.

58. Wein S, Ukropec J, Gasperikova D, Klimes I, Sebokova E. Concerted action of leptin in regulation of fatty acid oxidation in skeletal muscle and liver. Exp Clin Endocrinol Diabetes 2007;115(4):244-51.

59. Muoio DM, Dohm GL, Fiedorek FT, Jr., Tapscott EB, Coleman RA. Leptin directly alters lipid partitioning in skeletal muscle. Diabetes 1997;46(8):1360-3.

60. Shimabukuro M, Koyama K, Chen G, et al. Direct antidiabetic effect of leptin through triglyceride depletion of tissues. Proc Natl Acad Sci U S A 1997;94(9):4637-41.

61. Considine RV, Sinha MK, Heiman ML, et al. Serum immunoreactive-leptin concentrations in normal-weight and obese humans. N Engl J Med 1996;334(5):292-5.

62. Segal KR, Landt M, Klein S. Relationship between insulin sensitivity and plasma leptin concentration in lean and obese men. Diabetes 1996;45(7):988-91.

63. Ronti T, Lupattelli G, Mannarino E. The endocrine function of adipose tissue: an update. Clin Endocrinol (Oxf) 2006;64(4):355-65.

64. Yamauchi T, Nio Y, Maki T, et al. Targeted disruption of AdipoR1 and AdipoR2 causes abrogation of adiponectin binding and metabolic actions. Nat Med 2007;13(3):332-9.

65. Tomas E, Tsao TS, Saha AK, et al. Enhanced muscle fat oxidation and glucose transport by ACRP30 globular domain: acetyl-CoA carboxylase inhibition and AMP-activated protein kinase activation. Proc Natl Acad Sci U S A 2002;99(25):16309-13.

66. Yamauchi T, Kamon J, Ito Y, et al. Cloning of adiponectin receptors that mediate antidiabetic metabolic effects. Nature 2003;423(6941):762-9.

67. Yamauchi T, Kamon J, Minokoshi Y, et al. Adiponectin stimulates glucose utilization and fattyacid oxidation by activating AMP-activated protein kinase. Nat Med 2002;8(11):1288-95.

68. Ceddia RB, Somwar R, Maida A, Fang X, Bikopoulos G, Sweeney G. Globular adiponectin increases GLUT4 translocation and glucose uptake but reduces glycogen synthesis in rat skeletal muscle cells. Diabetologia 2005;48(1):132-9.

69. Mao X, Kikani CK, Riojas RA, et al. APPL1 binds to adiponectin receptors and mediates adiponectin signalling and function. Nat Cell Biol 2006;8(5):516-23.

70. Tilg $\mathrm{H}$, Moschen AR. Adipocytokines: mediators linking adipose tissue, inflammation and immunity. Nat Rev Immunol 2006;6(10):772-83.

71. Souza SC, Palmer HJ, Kang YH, et al. TNF-alpha induction of lipolysis is mediated through activation of the extracellular signal related kinase pathway in 3T3-L1 adipocytes. J Cell Biochem 2003;89(6):1077-86.

72. Zhang HH, Halbleib M, Ahmad F, Manganiello VC, Greenberg AS. Tumor necrosis factoralpha stimulates lipolysis in differentiated human adipocytes through activation of extracellular signal-related kinase and elevation of intracellular cAMP. Diabetes 2002;51(10):2929-35.

73. van Hall G, Steensberg A, Sacchetti M, et al. Interleukin-6 stimulates lipolysis and fat oxidation in humans. J Clin Endocrinol Metab 2003;88(7):3005-10. 
74. de Alvaro C, Teruel T, Hernandez R, Lorenzo M. Tumor necrosis factor alpha produces insulin resistance in skeletal muscle by activation of inhibitor kappaB kinase in a p38 MAPKdependent manner. J Biol Chem 2004;279(17):17070-8.

75. Hosogai N, Fukuhara A, Oshima K, et al. Adipose tissue hypoxia in obesity and its impact on adipocytokine dysregulation. Diabetes 2007;56(4):901-11.

76. Greijer AE, van der Wall E. The role of hypoxia inducible factor 1 (HIF-1) in hypoxia induced apoptosis. J Clin Pathol 2004;57(10):1009-14.

77. Goossens GH, Bizzarri A, Venteclef N, et al. Increased adipose tissue oxygen tension in obese compared with lean men is accompanied by insulin resistance, impaired adipose tissue capillarization, and inflammation. Circulation 2011;124(1):67-76.

78. Gregor MF, Hotamisligil GS. Thematic review series: Adipocyte Biology. Adipocyte stress: the endoplasmic reticulum and metabolic disease. J Lipid Res 2007;48(9):1905-14.

79. Houstis N, Rosen ED, Lander ES. Reactive oxygen species have a causal role in multiple forms of insulin resistance. Nature 2006;440(7086):944-8.

80. Cinti S, Mitchell G, Barbatelli G, et al. Adipocyte death defines macrophage localization and function in adipose tissue of obese mice and humans. J Lipid Res 2005;46(11):2347-55.

81. Kanda $\mathrm{H}$, Tateya S, Tamori $\mathrm{Y}$, et al. MCP-1 contributes to macrophage infiltration into adipose tissue, insulin resistance, and hepatic steatosis in obesity. J Clin Invest 2006;116(6):1494505.

82. Mantovani A, Sica A, Sozzani S, Allavena P, Vecchi A, Locati M. The chemokine system in diverse forms of macrophage activation and polarization. Trends Immunol 2004;25(12):67786.

83. Nguyen MT, Favelyukis S, Nguyen AK, et al. A subpopulation of macrophages infiltrates hypertrophic adipose tissue and is activated by free fatty acids via Toll-like receptors 2 and 4 and JNK-dependent pathways. J Biol Chem 2007;282(48):35279-92.

84. Lumeng CN, Bodzin JL, Saltiel AR. Obesity induces a phenotypic switch in adipose tissue macrophage polarization. J Clin Invest 2007;117(1):175-84.

85. Shi H, Kokoeva MV, Inouye K, Tzameli I, Yin H, Flier JS. TLR4 links innate immunity and fatty acid-induced insulin resistance. J Clin Invest 2006;116(11):3015-25.

86. Caspar-Bauguil S, Cousin B, Galinier A, et al. Adipose tissues as an ancestral immune organ: site-specific change in obesity. FEBS Lett 2005;579(17):3487-92.

87. Kintscher $U$, Hartge M, Hess K, et al. T-lymphocyte infiltration in visceral adipose tissue: a primary event in adipose tissue inflammation and the development of obesity-mediated insulin resistance. Arterioscler Thromb Vasc Biol 2008;28(7):1304-10.

88. Romeo GR, Lee J, Shoelson SE. Metabolic syndrome, insulin resistance, and roles of inflammation - mechanisms and therapeutic targets. Arterioscler Thromb Vasc Biol 2012;32(8):1771-6.

89. Ferrannini E. The theoretical bases of indirect calorimetry: a review. Metabolism 1988;37(3):287-301.

90. Wolfe R. Radioactive and Stable Isotope Tracers in Biomedicine. Principles and Practice of Kinetic Analysis. . Wiley-Liss, Inc, New York 1992.

91. Enoksson S, Degerman E, Hagstrom-Toft E, Large V, Arner P. Various phosphodiesterase subtypes mediate the in vivo antilipolytic effect of insulin on adipose tissue and skeletal muscle in man. Diabetologia 1998;41(5):560-8.

92. Jensen MD. Lipolysis: contribution from regional fat. Annu Rev Nutr 1997;17:127-39.

93. Blaak EE, Wagenmakers AJ, Glatz JF, et al. Plasma FFA utilization and fatty acid-binding protein content are diminished in type 2 diabetic muscle. Am J Physiol Endocrinol Metab 2000;279(1):E146-54.

94. Holloway GP, Luiken JJ, Glatz JF, Spriet LL, Bonen A. Contribution of FAT/CD36 to the regulation of skeletal muscle fatty acid oxidation: an overview. Acta Physiol (Oxf) 2008;194(4):293-309.

95. Bonen A, Luiken JJ, Arumugam Y, Glatz JF, Tandon NN. Acute regulation of fatty acid uptake involves the cellular redistribution of fatty acid translocase. J Biol Chem 2000;275(19):145018.

96. Bonen A, Luiken JJ, Liu S, et al. Palmitate transport and fatty acid transporters in red and white muscles. Am J Physiol 1998;275(3 Pt 1):E471-8.

97. Abumrad N, Harmon C, Ibrahimi A. Membrane transport of long-chain fatty acids: evidence for a facilitated process. J Lipid Res 1998;39(12):2309-18.

98. Schwieterman W, Sorrentino D, Potter BJ, et al. Uptake of oleate by isolated rat adipocytes is mediated by a $40-\mathrm{kDa}$ plasma membrane fatty acid binding protein closely related to that in liver and gut. Proc Natl Acad Sci U S A 1988;85(2):359-63. 
99. Gimeno RE, Ortegon AM, Patel S, et al. Characterization of a heart-specific fatty acid transport protein. J Biol Chem 2003;278(18):16039-44.

100. Hirsch D, Stahl A, Lodish HF. A family of fatty acid transporters conserved from mycobacterium to man. Proc Natl Acad Sci U S A 1998;95(15):8625-9.

101. Luiken JJ, Schaap FG, van Nieuwenhoven FA, van der Vusse GJ, Bonen A, Glatz JF. Cellular fatty acid transport in heart and skeletal muscle as facilitated by proteins. Lipids 1999;34 Suppl:S169-75.

102. Koonen DP, Glatz JF, Bonen A, Luiken JJ. Long-chain fatty acid uptake and FAT/CD36 translocation in heart and skeletal muscle. Biochim Biophys Acta 2005;1736(3):163-80.

103. Nickerson JG, Momken I, Benton CR, et al. Protein-mediated fatty acid uptake: regulation by contraction, AMP-activated protein kinase, and endocrine signals. Appl Physiol Nutr Metab 2007;32(5):865-73.

104. Hajri T, Han XX, Bonen A, Abumrad NA. Defective fatty acid uptake modulates insulin responsiveness and metabolic responses to diet in CD36-null mice. J Clin Invest 2002;109(10):1381-9.

105. Bonen A, Chabowski A, Luiken JJ, Glatz JF. Is membrane transport of FFA mediated by lipid, protein, or both? Mechanisms and regulation of protein-mediated cellular fatty acid uptake: molecular, biochemical, and physiological evidence. Physiology (Bethesda) 2007;22:15-29.

106. Bonen A, Parolin ML, Steinberg GR, et al. Triacylglycerol accumulation in human obesity and type 2 diabetes is associated with increased rates of skeletal muscle fatty acid transport and increased sarcolemmal FAT/CD36. Faseb J 2004;18(10):1144-6.

107. Kelley DE, Goodpaster B, Wing RR, Simoneau JA. Skeletal muscle fatty acid metabolism in association with insulin resistance, obesity, and weight loss. Am J Physiol 1999;277(6 Pt 1):E1130-41.

108. Corpeleijn E, Mensink M, Kooi ME, Roekaerts PM, Saris WH, Blaak EE. Impaired skeletal muscle substrate oxidation in glucose-intolerant men improves after weight loss. Obesity (Silver Spring) 2008;16(5):1025-32.

109. Moors CC, van der Zijl NJ, Diamant M, Blaak EE, Goossens GH. Impaired insulin sensitivity is accompanied by disturbances in skeletal muscle fatty acid handling in subjects with impaired glucose metabolism. Int J Obes (Lond) 2012;36(5):709-17.

110. Jocken JW, Roepstorff C, Goossens GH, et al. Hormone-sensitive lipase serine phosphorylation and glycerol exchange across skeletal muscle in lean and obese subjects: effect of beta-adrenergic stimulation. Diabetes 2008;57(7):1834-41.

111. Evans K, Burdge GC, Wootton SA, Clark ML, Frayn KN. Regulation of dietary fatty acid entrapment in subcutaneous adipose tissue and skeletal muscle. Diabetes 2002;51(9):268490.

112. Teusink B, Voshol PJ, Dahlmans VE, et al. Contribution of fatty acids released from lipolysis of plasma triglycerides to total plasma fatty acid flux and tissue-specific fatty acid uptake. Diabetes 2003;52(3):614-20.

113. Lithell H, Boberg J, Hellsing K, Lundqvist G, Vessby B. Lipoprotein-lipase activity in human skeletal muscle and adipose tissue in the fasting and the fed states. Atherosclerosis 1978;30(1):89-94.

114. Farese RV, Jr., Yost TJ, Eckel RH. Tissue-specific regulation of lipoprotein lipase activity by insulin/glucose in normal-weight humans. Metabolism 1991;40(2):214-6.

115. Lopez V, Saraff K, Medh JD. Down-regulation of lipoprotein lipase increases glucose uptake in L6 muscle cells. Biochem Biophys Res Commun 2009;389(1):34-9.

116. Kim JK, Fillmore JJ, Chen Y, et al. Tissue-specific overexpression of lipoprotein lipase causes tissue-specific insulin resistance. Proc Natl Acad Sci U S A 2001;98(13):7522-7.

117. Jeukendrup AE. Regulation of fat metabolism in skeletal muscle. Ann N Y Acad Sci 2002;967:217-35.

118. Coleman RA, Lee DP. Enzymes of triacylglycerol synthesis and their regulation. Prog Lipid Res 2004;43(2):134-76.

119. Bagnato C, Igal RA. Overexpression of diacylglycerol acyltransferase-1 reduces phospholipid synthesis, proliferation, and invasiveness in simian virus 40-transformed human lung fibroblasts. J Biol Chem 2003;278(52):52203-11.

120. Liu L, Zhang Y, Chen N, Shi X, Tsang B, Yu YH. Upregulation of myocellular DGAT1 augments triglyceride synthesis in skeletal muscle and protects against fat-induced insulin resistance. J Clin Invest 2007;117(6):1679-89.

121. Timmers S, de Vogel-van den Bosch J, Hesselink MK, et al. Paradoxical increase in TAG and DAG content parallel the insulin sensitizing effect of unilateral DGAT1 overexpression in rat skeletal muscle. PLoS One 2011;6(1):e14503. 
122. Jocken JW, Moro C, Goossens GH, et al. Skeletal muscle lipase content and activity in obesity and type 2 diabetes. J Clin Endocrinol Metab 2010;95(12):5449-53.

123. Kelley DE, Mandarino LJ. Fuel selection in human skeletal muscle in insulin resistance: a reexamination. Diabetes 2000;49(5):677-83.

124. Corpeleijn E, Saris WH, Blaak EE. Metabolic flexibility in the development of insulin resistance and type 2 diabetes: effects of lifestyle. Obes Rev 2009;10(2):178-93.

125. Kelley DE, Simoneau JA. Impaired free fatty acid utilization by skeletal muscle in non-insulindependent diabetes mellitus. J Clin Invest 1994;94(6):2349-56.

126. Blaak EE, van Aggel-Leijssen DP, Wagenmakers AJ, Saris WH, van Baak MA. Impaired oxidation of plasma-derived fatty acids in type 2 diabetic subjects during moderate-intensity exercise. Diabetes 2000;49(12):2102-7.

127. Blaak EE, Van Baak MA, Kemerink GJ, Pakbiers MT, Heidendal GA, Saris WH. Betaadrenergic stimulation of energy expenditure and forearm skeletal muscle metabolism in lean and obese men. Am J Physiol 1994;267(2 Pt 1):E306-15.

128. Price NT, Jackson VN, van der Leij FR, et al. Cloning and expression of the liver and muscle isoforms of ovine carnitine palmitoyltransferase 1: residues within the $\mathrm{N}$-terminus of the muscle isoform influence the kinetic properties of the enzyme. Biochem J 2003;372(Pt 3):8719.

129. Consitt LA, Bell JA, Houmard JA. Intramuscular lipid metabolism, insulin action, and obesity. IUBMB Life 2009;61(1):47-55.

130. McGarry JD, Brown NF. The mitochondrial carnitine palmitoyltransferase system. From concept to molecular analysis. Eur J Biochem 1997;244(1):1-14.

131. Patil PB, Minteer SD, Mielke AA, et al. Malonyl coenzyme A affects insulin-stimulated glucose transport in myotubes. Arch Physiol Biochem 2007;113(1):13-24.

132. Park H, Kaushik VK, Constant S, et al. Coordinate regulation of malonyl-CoA decarboxylase, sn-glycerol-3-phosphate acyltransferase, and acetyl-CoA carboxylase by AMP-activated protein kinase in rat tissues in response to exercise. J Biol Chem 2002;277(36):32571-7.

133. Rasmussen BB, Holmback UC, Volpi E, Morio-Liondore B, Paddon-Jones D, Wolfe RR. Malonyl coenzyme A and the regulation of functional carnitine palmitoyltransferase-1 activity and fat oxidation in human skeletal muscle. J Clin Invest 2002;110(11):1687-93.

134. Hardie DG. AMP-activated/SNF1 protein kinases: conserved guardians of cellular energy. Nat Rev Mol Cell Biol 2007;8(10):774-85.

135. Winder WW, Hardie DG. Inactivation of acetyl-CoA carboxylase and activation of AMPactivated protein kinase in muscle during exercise. Am J Physiol 1996;270(2 Pt 1):E299-304.

136. Saha AK, Schwarsin AJ, Roduit R, et al. Activation of malonyl-CoA decarboxylase in rat skeletal muscle by contraction and the AMP-activated protein kinase activator 5aminoimidazole-4-carboxamide-1-beta -D-ribofuranoside. J Biol Chem 2000;275(32):2427983.

137. Campbell SE, Tandon NN, Woldegiorgis G, Luiken JJ, Glatz JF, Bonen A. A novel function for fatty acid translocase (FAT)/CD36: involvement in long chain fatty acid transfer into the mitochondria. J Biol Chem 2004;279(35):36235-41.

138. Bezaire V, Bruce CR, Heigenhauser GJ, et al. Identification of fatty acid translocase on human skeletal muscle mitochondrial membranes: essential role in fatty acid oxidation. Am J Physiol Endocrinol Metab 2006;290(3):E509-15.

139. Schenk S, Horowitz JF. Coimmunoprecipitation of FAT/CD36 and CPT I in skeletal muscle increases proportionally with fat oxidation after endurance exercise training. Am J Physiol Endocrinol Metab 2006;291(2):E254-60.

140. Berk PD, Wada H, Horio $\mathrm{Y}$, et al. Plasma membrane fatty acid-binding protein and mitochondrial glutamic-oxaloacetic transaminase of rat liver are related. Proc Natl Acad Sci U S A 1990;87(9):3484-8.

141. Stump J, Rash G, Semon J, Christian W, Miller K. A comparison of two modes of cervical exercise in adolescent male athletes. J Manipulative Physiol Ther 1993;16(3):155-60.

142. Sivitz WI, Yorek MA. Mitochondrial dysfunction in diabetes: from molecular mechanisms to functional significance and therapeutic opportunities. Antioxid Redox Signal 2010;12(4):53777.

143. Kraegen EW, Cooney GJ, Turner N. Muscle insulin resistance: a case of fat overconsumption, not mitochondrial dysfunction. Proc Natl Acad Sci U S A 2008;105(22):7627-8.

144. Phielix E, Schrauwen-Hinderling VB, Mensink M, et al. Lower intrinsic ADP-stimulated mitochondrial respiration underlies in vivo mitochondrial dysfunction in muscle of male type 2 diabetic patients. Diabetes 2008;57(11):2943-9. 
145. Schrauwen-Hinderling VB, Kooi ME, Hesselink MK, et al. Impaired in vivo mitochondrial function but similar intramyocellular lipid content in patients with type 2 diabetes mellitus and BMI-matched control subjects. Diabetologia 2007;50(1):113-20.

146. Kelley DE, He J, Menshikova EV, Ritov VB. Dysfunction of mitochondria in human skeletal muscle in type 2 diabetes. Diabetes 2002;51(10):2944-50.

147. Mogensen M, Sahlin K, Fernstrom M, et al. Mitochondrial respiration is decreased in skeletal muscle of patients with type 2 diabetes. Diabetes 2007;56(6):1592-9.

148. Stump CS, Short KR, Bigelow ML, Schimke JM, Nair KS. Effect of insulin on human skeletal muscle mitochondrial ATP production, protein synthesis, and mRNA transcripts. Proc Natl Acad Sci U S A 2003;100(13):7996-8001.

149. Szendroedi J, Schmid Al, Chmelik M, et al. Muscle mitochondrial ATP synthesis and glucose transport/phosphorylation in type 2 diabetes. PLoS Med 2007;4(5):e154.

150. Patti ME, Butte AJ, Crunkhorn S, et al. Coordinated reduction of genes of oxidative metabolism in humans with insulin resistance and diabetes: Potential role of PGC1 and NRF1. Proc Natl Acad Sci U S A 2003;100(14):8466-71.

151. Mootha VK, Lindgren CM, Eriksson KF, et al. PGC-1alpha-responsive genes involved in oxidative phosphorylation are coordinately downregulated in human diabetes. Nat Genet 2003;34(3):267-73.

152. Morino K, Petersen KF, Dufour S, et al. Reduced mitochondrial density and increased IRS-1 serine phosphorylation in muscle of insulin-resistant offspring of type 2 diabetic parents. J Clin Invest 2005;115(12):3587-93.

153. Lin J, Handschin C, Spiegelman BM. Metabolic control through the PGC-1 family of transcription coactivators. Cell Metab 2005;1(6):361-70.

154. Koves TR, Ussher JR, Noland RC, et al. Mitochondrial overload and incomplete fatty acid oxidation contribute to skeletal muscle insulin resistance. Cell Metab 2008;7(1):45-56.

155. Mihalik SJ, Goodpaster BH, Kelley DE, et al. Increased levels of plasma acylcarnitines in obesity and type 2 diabetes and identification of a marker of glucolipotoxicity. Obesity (Silver Spring) 2010;18(9):1695-700.

156. Randle PJ, Garland PB, Hales CN, Newsholme EA. The glucose fatty-acid cycle. Its role in insulin sensitivity and the metabolic disturbances of diabetes mellitus. Lancet 1963;1(7285):785-9.

157. Shulman GI, Rothman DL, Jue T, Stein P, DeFronzo RA, Shulman RG. Quantitation of muscle glycogen synthesis in normal subjects and subjects with non-insulin-dependent diabetes by 13C nuclear magnetic resonance spectroscopy. N Engl J Med 1990;322(4):2238.

158. Rothman DL, Shulman RG, Shulman GI. 31P nuclear magnetic resonance measurements of muscle glucose-6-phosphate. Evidence for reduced insulin-dependent muscle glucose transport or phosphorylation activity in non-insulin-dependent diabetes mellitus. J Clin Invest 1992;89(4):1069-75.

159. Rothman DL, Magnusson I, Cline G, et al. Decreased muscle glucose transport/phosphorylation is an early defect in the pathogenesis of non-insulin-dependent diabetes mellitus. Proc Natl Acad Sci U S A 1995;92(4):983-7.

160. Shulman Gl. Cellular mechanisms of insulin resistance. J Clin Invest 2000;106(2):171-6.

161. Boden G, Lebed B, Schatz M, Homko C, Lemieux S. Effects of acute changes of plasma free fatty acids on intramyocellular fat content and insulin resistance in healthy subjects. Diabetes 2001;50(7):1612-7.

162. Roden M, Price TB, Perseghin G, et al. Mechanism of free fatty acid-induced insulin resistance in humans. J Clin Invest 1996;97(12):2859-65.

163. Krssak M, Falk Petersen K, Dresner A, et al. Intramyocellular lipid concentrations are correlated with insulin sensitivity in humans: a $1 \mathrm{H}$ NMR spectroscopy study. Diabetologia 1999;42(1):113-6.

164. Jacob S, Machann J, Rett K, et al. Association of increased intramyocellular lipid content with insulin resistance in lean nondiabetic offspring of type 2 diabetic subjects. Diabetes 1999;48(5):1113-9.

165. Goodpaster BH, He J, Watkins S, Kelley DE. Skeletal muscle lipid content and insulin resistance: evidence for a paradox in endurance-trained athletes. J Clin Endocrinol Metab 2001;86(12):5755-61.

166. Schenk S, Horowitz JF. Acute exercise increases triglyceride synthesis in skeletal muscle and prevents fatty acid-induced insulin resistance. J Clin Invest 2007;117(6):1690-8.

167. Idris I, Gray S, Donnelly R. Protein kinase C activation: isozyme-specific effects on metabolism and cardiovascular complications in diabetes. Diabetologia 2001;44(6):659-73. 
168. Schmitz-Peiffer C, Browne CL, Oakes ND, et al. Alterations in the expression and cellular localization of protein kinase $C$ isozymes epsilon and theta are associated with insulin resistance in skeletal muscle of the high-fat-fed rat. Diabetes 1997;46(2):169-78.

169. Yu C, Chen Y, Cline GW, et al. Mechanism by which fatty acids inhibit insulin activation of insulin receptor substrate-1 (IRS-1)-associated phosphatidylinositol 3-kinase activity in muscle. J Biol Chem 2002;277(52):50230-6.

170. Itani SI, Zhou Q, Pories WJ, MacDonald KG, Dohm GL. Involvement of protein kinase C in human skeletal muscle insulin resistance and obesity. Diabetes 2000;49(8):1353-8.

171. Itani SI, Pories WJ, Macdonald KG, Dohm GL. Increased protein kinase C theta in skeletal muscle of diabetic patients. Metabolism 2001;50(5):553-7.

172. Itani SI, Ruderman NB, Schmieder F, Boden G. Lipid-induced insulin resistance in human muscle is associated with changes in diacylglycerol, protein kinase C, and IkappaB-alpha. Diabetes 2002;51(7):2005-11.

173. Goni FM, Alonso A. Structure and functional properties of diacylglycerols in membranes. Prog Lipid Res 1999;38(1):1-48.

174. Wakelam MJ. Diacylglycerol--when is it an intracellular messenger? Biochim Biophys Acta 1998;1436(1-2):117-26.

175. Boni LT, Rando RR. The nature of protein kinase $\mathrm{C}$ activation by physically defined phospholipid vesicles and diacylglycerols. J Biol Chem 1985;260(19):10819-25.

176. Bergman BC, Perreault L, Hunerdosse DM, Koehler MC, Samek AM, Eckel RH. Increased intramuscular lipid synthesis and low saturation relate to insulin sensitivity in endurancetrained athletes. J Appl Physiol 2010;108(5):1134-41.

177. Kuerschner L, Moessinger C, Thiele C. Imaging of lipid biosynthesis: how a neutral lipid enters lipid droplets. Traffic 2008;9(3):338-52.

178. Bergman BC, Hunerdosse DM, Kerege A, Playdon MC, Perreault L. Localisation and composition of skeletal muscle diacylglycerol predicts insulin resistance in humans. Diabetologia 2012;55(4):1140-50.

179. Hannun YA, Obeid LM. The Ceramide-centric universe of lipid-mediated cell regulation: stress encounters of the lipid kind. J Biol Chem 2002;277(29):25847-50.

180. Merrill AH, Jr., Jones DD. An update of the enzymology and regulation of sphingomyelin metabolism. Biochim Biophys Acta 1990;1044(1):1-12.

181. Long SD, Pekala PH. Lipid mediators of insulin resistance: ceramide signalling downregulates GLUT4 gene transcription in 3T3-L1 adipocytes. Biochem J 1996;319 ( Pt 1):17984.

182. Chavez JA, Knotts TA, Wang LP, et al. A role for ceramide, but not diacylglycerol, in the antagonism of insulin signal transduction by saturated fatty acids. $\mathrm{J}$ Biol Chem 2003;278(12):10297-303.

183. Ellis BA, Poynten A, Lowy AJ, et al. Long-chain acyl-CoA esters as indicators of lipid metabolism and insulin sensitivity in rat and human muscle. Am J Physiol Endocrinol Metab 2000;279(3):E554-60.

184. Bessesen DH, Bull S, Cornier MA. Trafficking of dietary fat and resistance to obesity. Physiol Behav 2008;94(5):681-8.

185. Bray GA. Treatment for obesity: a nutrient balance/nutrient partition approach. Nutr Rev 1991;49(2):33-45.

186. Acheson KJ, Schutz Y, Bessard T, Anantharaman K, Flatt JP, Jequier E. Glycogen storage capacity and de novo lipogenesis during massive carbohydrate overfeeding in man. Am J Clin Nutr 1988;48(2):240-7.

187. Schutz Y, Flatt JP, Jequier E. Failure of dietary fat intake to promote fat oxidation: a factor favoring the development of obesity. Am J Clin Nutr 1989;50(2):307-14.

188. Schrauwen $\mathrm{P}$, Westerterp KR. The role of high-fat diets and physical activity in the regulation of body weight. Br J Nutr 2000;84(4):417-27.

189. Thomas CD, Peters JC, Reed GW, Abumrad NN, Sun M, Hill JO. Nutrient balance and energy expenditure during ad libitum feeding of high-fat and high-carbohydrate diets in humans. Am J Clin Nutr 1992;55(5):934-42.

190. Flatt JP, Ravussin E, Acheson KJ, Jequier E. Effects of dietary fat on postprandial substrate oxidation and on carbohydrate and fat balances. J Clin Invest 1985;76(3):1019-24.

191. Galgani J, Ravussin E. Energy metabolism, fuel selection and body weight regulation. Int J Obes (Lond) 2008;32 Suppl 7:S109-19.

192. Astrup A. The relevance of increased fat oxidation for body-weight management: metabolic inflexibility in the predisposition to weight gain. Obes Rev 2011;12(10):859-65. 
193. Flatt JP. The difference in the storage capacities for carbohydrate and for fat, and its implications in the regulation of body weight. Ann N Y Acad Sci 1987;499:104-23.

194. Schrauwen P, Lichtenbelt WD, Saris WH, Westerterp KR. Fat balance in obese subjects: role of glycogen stores. Am J Physiol 1998;274(6 Pt 1):E1027-33.

195. Astrup A, Buemann B, Western P, Toubro S, Raben A, Christensen NJ. Obesity as an adaptation to a high-fat diet: evidence from a cross-sectional study. Am J Clin Nutr 1994;59(2):350-5.

196. Saris WH, Asp NG, Bjorck I, et al. Functional food science and substrate metabolism. Br J Nutr 1998;80 Suppl 1:S47-75.

197. Wing RR, Blair EH, Bononi P, Marcus MD, Watanabe R, Bergman RN. Caloric restriction per se is a significant factor in improvements in glycemic control and insulin sensitivity during weight loss in obese NIDDM patients. Diabetes Care 1994;17(1):30-6.

198. Klein S, Sheard NF, Pi-Sunyer X, et al. Weight management through lifestyle modification for the prevention and management of type 2 diabetes: rationale and strategies. A statement of the American Diabetes Association, the North American Association for the Study of Obesity, and the American Society for Clinical Nutrition. Am J Clin Nutr 2004;80(2):257-63.

199. Goodpaster BH, Katsiaras A, Kelley DE. Enhanced fat oxidation through physical activity is associated with improvements in insulin sensitivity in obesity. Diabetes 2003;52(9):2191-7.

200. Raben A, Andersen HB, Christensen NJ, Madsen J, Holst JJ, Astrup A. Evidence for an abnormal postprandial response to a high-fat meal in women predisposed to obesity. Am J Physiol 1994;267(4 Pt 1):E549-59.

201. Gray RE, Tanner CJ, Pories WJ, MacDonald KG, Houmard JA. Effect of weight loss on muscle lipid content in morbidly obese subjects. Am $J$ Physiol Endocrinol Metab 2003;284(4):E726-32.

202. Goodpaster BH, Theriault R, Watkins SC, Kelley DE. Intramuscular lipid content is increased in obesity and decreased by weight loss. Metabolism 2000;49(4):467-72.

203. Civitarese AE, Carling S, Heilbronn LK, et al. Calorie restriction increases muscle mitochondrial biogenesis in healthy humans. PLoS Med 2007;4(3):e76.

204. Nemoto S, Fergusson MM, Finkel T. SIRT1 functionally interacts with the metabolic regulator and transcriptional coactivator PGC-1\{alpha\}. J Biol Chem 2005;280(16):16456-60.

205. Rodgers JT, Lerin C, Haas W, Gygi SP, Spiegelman BM, Puigserver P. Nutrient control of glucose homeostasis through a complex of PGC-1alpha and SIRT1. Nature 2005;434(7029):113-8.

206. Hernandez-Alvarez MI, Chiellini C, Manco M, et al. Genes involved in mitochondrial biogenesis/function are induced in response to bilio-pancreatic diversion in morbidly obese individuals with normal glucose tolerance but not in type 2 diabetic patients. Diabetologia 2009;52(8):1618-27.

207. Gastaldi G, Russell A, Golay A, et al. Upregulation of peroxisome proliferator-activated receptor gamma coactivator gene (PGC1A) during weight loss is related to insulin sensitivity but not to energy expenditure. Diabetologia 2007;50(11):2348-55.

208. Berggren JR, Boyle KE, Chapman WH, Houmard JA. Skeletal muscle lipid oxidation and obesity: influence of weight loss and exercise. Am $J$ Physiol Endocrinol Metab 2008;294(4):E726-32.

209. Sadruddin S, Arora R. Resveratrol: biologic and therapeutic implications. J Cardiometab Syndr 2009;4(2):102-6.

210. Halliwell B. Dietary polyphenols: good, bad, or indifferent for your health? Cardiovasc Res 2007;73(2):341-7.

211. Petersen KF, Dufour S, Befroy D, Garcia R, Shulman GI. Impaired mitochondrial activity in the insulin-resistant offspring of patients with type 2 diabetes. N Engl J Med 2004;350(7):66471.

212. Virkamaki A, Korsheninnikova E, Seppala-Lindroos A, et al. Intramyocellular lipid is associated with resistance to in vivo insulin actions on glucose uptake, antilipolysis, and early insulin signaling pathways in human skeletal muscle. Diabetes 2001;50(10):2337-43.

213. Lagouge M, Argmann C, Gerhart-Hines Z, et al. Resveratrol improves mitochondrial function and protects against metabolic disease by activating SIRT1 and PGC-1alpha. Cell 2006;127(6):1109-22.

214. Hegarty BD, Turner N, Cooney GJ, Kraegen EW. Insulin resistance and fuel homeostasis: the role of AMP-activated protein kinase. Acta Physiol (Oxf) 2009;196(1):129-45.

215. Canto C, Gerhart-Hines Z, Feige JN, et al. AMPK regulates energy expenditure by modulating NAD+ metabolism and SIRT1 activity. Nature 2009;458(7241):1056-60. 
216. Chi TC, Chen WP, Chi TL, et al. Phosphatidylinositol-3-kinase is involved in the antihyperglycemic effect induced by resveratrol in streptozotocin-induced diabetic rats. Life Sci 2007;80(18):1713-20.

217. Deng JY, Hsieh PS, Huang JP, Lu LS, Hung LM. Activation of estrogen receptor is crucial for resveratrol-stimulating muscular glucose uptake via both insulin-dependent and -independent pathways. Diabetes 2008;57(7):1814-23.

218. Park CE, Kim MJ, Lee JH, et al. Resveratrol stimulates glucose transport in C2C12 myotubes by activating AMP-activated protein kinase. Exp Mol Med 2007;39(2):222-9.

219. Brasnyo P, Molnar GA, Mohas M, et al. Resveratrol improves insulin sensitivity, reduces oxidative stress and activates the Akt pathway in type 2 diabetic patients. $\mathrm{Br} \mathrm{J}$ Nutr 2011;106(3):383-9.

220. Baur JA, Pearson KJ, Price NL, et al. Resveratrol improves health and survival of mice on a high-calorie diet. Nature 2006;444(7117):337-42.

221. Alberdi G, Rodriguez VM, Miranda J, et al. Changes in white adipose tissue metabolism induced by resveratrol in rats. Nutr Metab (Lond) 2011;8(1):29.

222. Szkudelska K, Nogowski L, Szkudelski T. Resveratrol, a naturally occurring diphenolic compound, affects lipogenesis, lipolysis and the antilipolytic action of insulin in isolated rat adipocytes. J Steroid Biochem Mol Biol 2009;113(1-2):17-24.

223. Lasa A, Schweiger $M$, Kotzbeck $P$, et al. Resveratrol regulates lipolysis via adipose triglyceride lipase. J Nutr Biochem 2012;23(4):379-84.

224. Picard F, Kurtev $M$, Chung $N$, et al. Sirt1 promotes fat mobilization in white adipocytes by repressing PPAR-gamma. Nature 2004;429(6993):771-6.

225. Ahn J, Lee H, Kim S, Ha T. Resveratrol inhibits TNF-alpha-induced changes of adipokines in 3T3-L1 adipocytes. Biochem Biophys Res Commun 2007;364(4):972-7.

226. Zhu J, Yong W, Wu X, et al. Anti-inflammatory effect of resveratrol on TNF-alpha-induced MCP-1 expression in adipocytes. Biochem Biophys Res Commun 2008;369(2):471-7.

227. Rivera L, Moron R, Zarzuelo A, Galisteo M. Long-term resveratrol administration reduces metabolic disturbances and lowers blood pressure in obese Zucker rats. Biochem Pharmacol 2009;77(6):1053-63.

228. Wellen KE, Hotamisligil GS. Inflammation, stress, and diabetes. J Clin Invest 2005;115(5):1111-9.

229. Qiao L, Shao J. SIRT1 regulates adiponectin gene expression through Foxo1-C/enhancerbinding protein alpha transcriptional complex. J Biol Chem 2006;281(52):39915-24.

230. Beaudeux JL, Nivet-Antoine V, Giral P. Resveratrol: a relevant pharmacological approach for the treatment of metabolic syndrome? Curr Opin Clin Nutr Metab Care 2010;13(6):729-36.

231. Riserus U, Willett WC, Hu FB. Dietary fats and prevention of type 2 diabetes. Prog Lipid Res 2009;48(1):44-51.

232. Mayer EJ, Newman B, Quesenberry CP, Jr., Selby JV. Usual dietary fat intake and insulin concentrations in healthy women twins. Diabetes Care 1993;16(11):1459-69.

233. Parker DR, Weiss ST, Troisi R, Cassano PA, Vokonas PS, Landsberg L. Relationship of dietary saturated fatty acids and body habitus to serum insulin concentrations: the Normative Aging Study. Am J Clin Nutr 1993;58(2):129-36.

234. Marshall JA, Bessesen DH, Hamman RF. High saturated fat and low starch and fibre are associated with hyperinsulinaemia in a non-diabetic population: the San Luis Valley Diabetes Study. Diabetologia 1997;40(4):430-8.

235. Vessby $B$, Uusitupa $M$, Hermansen $K$, et al. Substituting dietary saturated for monounsaturated fat impairs insulin sensitivity in healthy men and women: The KANWU Study. Diabetologia 2001;44(3):312-9.

236. Summers LK, Fielding BA, Bradshaw HA, et al. Substituting dietary saturated fat with polyunsaturated fat changes abdominal fat distribution and improves insulin sensitivity. Diabetologia 2002;45(3):369-77.

237. Tierney AC, McMonagle J, Shaw DI, et al. Effects of dietary fat modification on insulin sensitivity and on other risk factors of the metabolic syndrome--LIPGENE: a European randomized dietary intervention study. Int J Obes (Lond) 2011;35(6):800-9.

238. Jebb SA, Lovegrove JA, Griffin BA, et al. Effect of changing the amount and type of fat and carbohydrate on insulin sensitivity and cardiovascular risk: the RISCK (Reading, Imperial, Surrey, Cambridge, and Kings) trial. Am J Clin Nutr 2010;92(4):748-58.

239. Lee JS, Pinnamaneni SK, Eo SJ, et al. Saturated, but not $n-6$ polyunsaturated, fatty acids induce insulin resistance: role of intramuscular accumulation of lipid metabolites. J Appl Physiol 2006;100(5):1467-74. 
240. Gaster M, Rustan AC, Beck-Nielsen H. Differential utilization of saturated palmitate and unsaturated oleate: evidence from cultured myotubes. Diabetes 2005;54(3):648-56.

241. Piers LS, Walker KZ, Stoney RM, Soares MJ, O'Dea K. Substitution of saturated with monounsaturated fat in a 4-week diet affects body weight and composition of overweight and obese men. Br J Nutr 2003;90(3):717-27.

242. Casas-Agustench P, Lopez-Uriarte P, Bullo M, Ros E, Gomez-Flores A, Salas-Salvado J. Acute effects of three high-fat meals with different fat saturations on energy expenditure, substrate oxidation and satiety. Clin Nutr 2009;28(1):39-45.

243. DeLany JP, Windhauser MM, Champagne CM, Bray GA. Differential oxidation of individual dietary fatty acids in humans. Am J Clin Nutr 2000;72(4):905-11.

244. MacDougall DE, Jones PJ, Vogt J, Phang PT, Kitts DD. Utilization of myristic and palmitic acid in humans fed different dietary fats. Eur J Clin Invest 1996;26(9):755-62.

245. Sampath H, Ntambi JM. Polyunsaturated fatty acid regulation of genes of lipid metabolism. Annu Rev Nutr 2005;25:317-40.

246. Jans A, van Hees AM, Gjelstad IM, et al. Impact of dietary fat quantity and quality on skeletal muscle fatty acid metabolism in subjects with the metabolic syndrome. Metabolism 2012.

247. Borkman M, Storlien LH, Pan DA, Jenkins AB, Chisholm DJ, Campbell LV. The relation between insulin sensitivity and the fatty-acid composition of skeletal-muscle phospholipids. $\mathrm{N}$ Engl J Med 1993;328(4):238-44.

248. Hulbert AJ, Turner N, Storlien LH, Else PL. Dietary fats and membrane function: implications for metabolism and disease. Biol Rev Camb Philos Soc 2005;80(1):155-69.

249. Gasperikova D, Klimes I, Kolter T, et al. Glucose transport and insulin signaling in rat muscle and adipose tissue. Effect of lipid availability. Ann N Y Acad Sci 1997;827:144-57.

250. Montell E, Turini M, Marotta M, et al. DAG accumulation from saturated fatty acids desensitizes insulin stimulation of glucose uptake in muscle cells. Am J Physiol Endocrinol Metab 2001;280(2):E229-37.

251. Clandinin MT, Cheema S, Field CJ, Baracos VE. Dietary lipids influence insulin action. Ann N Y Acad Sci 1993;683:151-63.

252. Manco M, Calvani M, Mingrone G. Effects of dietary fatty acids on insulin sensitivity and secretion. Diabetes Obes Metab 2004;6(6):402-13.

253. Weigert $\mathrm{C}$, Brodbeck $\mathrm{K}$, Staiger $\mathrm{H}$, et al. Palmitate, but not unsaturated fatty acids, induces the expression of interleukin- 6 in human myotubes through proteasome-dependent activation of nuclear factor-kappaB. J Biol Chem 2004;279(23):23942-52.

254. Ajuwon KM, Spurlock ME. Palmitate activates the NF-kappaB transcription factor and induces IL-6 and TNFalpha expression in 3T3-L1 adipocytes. J Nutr 2005;135(8):1841-6.

255. Baer DJ, Judd JT, Clevidence BA, Tracy RP. Dietary fatty acids affect plasma markers of inflammation in healthy men fed controlled diets: a randomized crossover study. Am J Clin Nutr 2004;79(6):969-73.

256. Thies F, Miles EA, Nebe-von-Caron G, et al. Influence of dietary supplementation with longchain $n-3$ or $n-6$ polyunsaturated fatty acids on blood inflammatory cell populations and functions and on plasma soluble adhesion molecules in healthy adults. Lipids 2001;36(11):1183-93.

257. Slavin JL. Position of the American Dietetic Association: health implications of dietary fiber. J Am Diet Assoc 2008;108(10):1716-31.

258. Trowell H. Ischemic heart disease and dietary fiber. Am J Clin Nutr 1972;25(9):926-32.

259. Hallfrisch J, Tobin JD, Muller DC, Andres R. Fiber intake, age, and other coronary risk factors in men of the Baltimore Longitudinal Study (1959-1975). J Gerontol 1988;43(3):M64-8.

260. Jacobs DR, Jr., Marquart L, Slavin J, Kushi LH. Whole-grain intake and cancer: an expanded review and meta-analysis. Nutr Cancer 1998;30(2):85-96.

261. Wolever TM, Nguyen PM, Chiasson JL, et al. Determinants of diet glycemic index calculated retrospectively from diet records of 342 individuals with non-insulin-dependent diabetes mellitus. Am J Clin Nutr 1994;59(6):1265-9.

262. Blundell JE, Green S, Burley V. Carbohydrates and human appetite. Am J Clin Nutr 1994;59(3 Suppl):728S-34S.

263. Heaton KW. Food fibre as an obstacle to energy intake. Lancet 1973;2(7843):1418-21.

264. Biorklund M, van Rees A, Mensink RP, Onning G. Changes in serum lipids and postprandial glucose and insulin concentrations after consumption of beverages with beta-glucans from oats or barley: a randomised dose-controlled trial. European journal of clinical nutrition 2005;59(11):1272-81.

265. Brennan CS. Dietary fibre, glycaemic response, and diabetes. Molecular nutrition \& food research 2005;49(6):560-70. 
266. Juntunen KS, Niskanen LK, Liukkonen KH, Poutanen KS, Holst JJ, Mykkanen HM. Postprandial glucose, insulin, and incretin responses to grain products in healthy subjects. The American journal of clinical nutrition 2002;75(2):254-62.

267. Ulmius $M$, Johansson $A$, Onning $G$. The influence of dietary fibre source and gender on the postprandial glucose and lipid response in healthy subjects. European journal of nutrition 2009;48(7):395-402.

268. Kendall CW, Esfahani A, Hoffman AJ, et al. Effect of novel maize-based dietary fibers on postprandial glycemia and insulinemia. Journal of the American College of Nutrition 2008;27(6):711-8.

269. Saris WH. Sugars, energy metabolism, and body weight control. The American journal of clinical nutrition 2003;78(4):850S-7S.

270. Brand-Miller J, McMillan-Price J, Steinbeck K, Caterson I. Dietary glycemic index: health implications. Journal of the American College of Nutrition 2009;28 Suppl:446S-9S.

271. Brand-Miller JC, Holt SH, Pawlak DB, McMillan J. Glycemic index and obesity. Am J Clin Nutr 2002;76(1):281S-5S.

272. Lindstrom J, Peltonen M, Eriksson JG, et al. High-fibre, low-fat diet predicts long-term weight loss and decreased type 2 diabetes risk: the Finnish Diabetes Prevention Study. Diabetologia 2006;49(5):912-20.

273. Weickert MO, Mohlig M, Schofl C, et al. Cereal fiber improves whole-body insulin sensitivity in overweight and obese women. Diabetes Care 2006;29(4):775-80.

274. Lu ZX, Walker KZ, Muir JG, Mascara T, O'Dea K. Arabinoxylan fiber, a byproduct of wheat flour processing, reduces the postprandial glucose response in normoglycemic subjects. Am J Clin Nutr 2000;71(5):1123-8.

275. Pereira MA, Jacobs DR, Jr., Pins JJ, et al. Effect of whole grains on insulin sensitivity in overweight hyperinsulinemic adults. Am J Clin Nutr 2002;75(5):848-55.

276. Wanders AJ, van den Borne JJ, de Graaf C, et al. Effects of dietary fibre on subjective appetite, energy intake and body weight: a systematic review of randomized controlled trials. Obes Rev 2011;12(9):724-39.

277. Pereira MA, Ludwig DS. Dietary fiber and body-weight regulation. Observations and mechanisms. Pediatr Clin North Am 2001;48(4):969-80.

278. Cani PD, Dewever C, Delzenne NM. Inulin-type fructans modulate gastrointestinal peptides involved in appetite regulation (glucagon-like peptide-1 and ghrelin) in rats. $\mathrm{Br} \mathrm{J}$ Nutr 2004;92(3):521-6.

279. Darzi J, Frost GS, Robertson MD. Do SCFA have a role in appetite regulation? Proc Nutr Soc 2011;70(1):119-28.

280. Rigaud D, Ryttig KR, Angel LA, Apfelbaum M. Overweight treated with energy restriction and a dietary fibre supplement: a 6 -month randomized, double-blind, placebo-controlled trial. Int $\mathrm{J}$ Obes (Lond) 1990;14(9):763-9.

281. Pittler MH, Ernst E. Guar gum for body weight reduction: meta-analysis of randomized trials. Am J Med 2001;110(9):724-30.

282. Hays NP, Starling RD, Liu X, et al. Effects of an ad libitum low-fat, high-carbohydrate diet on body weight, body composition, and fat distribution in older men and women: a randomized controlled trial. Arch Intern Med 2004;164(2):210-7.

283. Boler BM, Serao MC, Bauer LL, et al. Digestive physiological outcomes related to polydextrose and soluble maize fibre consumption by healthy adult men. $\mathrm{Br} J$ Nutr 2011;106(12):1864-71. 


\title{
2
}

\section{Expression of genes involved \\ in lipid metabolism}

in impaired glucose tolerant men: impact of insulin stimulation and weight loss

\author{
Ellen Konings \\ Eva Corpeleijn \\ Freek G. Bouwman \\ Edwin C. Mariman \\ Ellen E. Blaak
}

Journal of Nutrigenetics and Nutrigenomics 2010; 3(1):9-17. 


\section{Abstract}

Background: The impaired glucose tolerant (IGT) state is characterized by insulin resistance. Disturbances in fatty acid (FA) metabolism may underlie this reduced insulin sensitivity.

Aim: The aim was to investigate whether the prediabetic state is accompanied by changes in expression of genes involved in FA handling during fasting and insulin mediated conditions and, secondly, to study the impact of weight loss.

Methods: Seven IGT and five normal glucose tolerant (NGT) men, comparable for age and BMI, participated in the study. Five IGT men followed a 12-week weight loss program. Muscle biopsies were taken and expression of six genes was investigated.

Results: Subjects had a reduction of $15.5 \pm 4.3 \mathrm{~kg}$ body weight. Baseline gene expression was not different between NGT and IGT men. After hyperinsulinemic clamp, there was an overall upregulation of PGC1 $\alpha$, SREBP-1c, SREBP-2 and $A C C-2$. For SREBP-2, the upregulation was more pronounced in IGT men $(p=0.049)$. Weight loss significantly increased insulin sensitivity by $71 \%$, which was not reflected in altered gene expression profiles.

Conclusion: SREBP-2 shows altered insulin responsiveness in IGT men compared with NGT men, whilst there were no differences in basal gene expression. 


\section{Introduction}

Type 2 diabetes mellitus (T2DM) is one of the major causes of morbidity and mortality in the world and its prevalence has rapidly been increasing with currently about 200 million patients worldwide (1). The impaired glucose tolerant (IGT) state represents an intermediate phase between the normal glucose tolerant (NGT) state and T2DM (2). Insulin resistance and $\beta$-cell dysfunction occur very early in the disease progress (3). Insulin resistant skeletal muscle is characterized by a reduced ability to oxidize lipids (4), an impaired switch between fat and carbohydrate oxidation in the postprandial state (metabolic inflexibility) $(4,5)$, despite elevated lipids in the circulation ('lipid overflow'), and by an accumulation of triglycerides within skeletal muscle $(5,6)$. This may lead to accumulation of lipid intermediates, such as diacylglycerols, ceramides and long-chain fatty acyl-CoA, that can be linked to defects in the insulin signalling cascade (7-9).

The metabolic and molecular mechanisms responsible for the reduced fat oxidation are not completely understood (10). It has been suggested that enzymes involved in fatty acid transport and metabolism are altered and that the metabolic profile of the insulin resistant muscle is oriented more towards fat storage than oxidation (11, 12). Acetyl-CoA carboxylase (ACC)-2 may play an important role in this diminished lipid oxidation (13). It has been shown that T2DM subjects have a significantly elevated ACC-2 expression in skeletal muscle (14). Meanwhile, ACC-2 mRNA expression is significantly reduced as result of lifestyle intervention in impaired glucose tolerant subjects (15) together with improvements in fat oxidation. Another potential mechanism controlling intramyocellular triglyceride (IMTG) content is the lipogenic pathway in muscle. SREBPs are central regulators of lipid biosynthesis (16). It has been shown that SREBP-1c expression was significantly reduced in the skeletal muscle of T2DM patients (17). SREBP-2 mainly activates genes involved in cholesterol synthesis in liver (18). The exact role of SREBP-2 in skeletal muscle metabolism remains to be elucidated. Furthermore, an impaired mitochondrial function or reduced content have been proposed as underlying defects in fat oxidation and insulin resistance $(19,20)$, although several recent studies shed doubt on this concept $(21,22)$. A set of genes involved in oxidative phosphorylation, including peroxisomal proliferator activator receptor $\gamma$ coactivator $1 \alpha(P G C 1 \alpha)$, has recently been found to be coordinately downregulated in the skeletal muscle of T2DM patients $(23,24)$. Finally, several studies have also indicated important defects in the capability of insulin to regulate gene expression in peripheral tissues in $\operatorname{T2DM}(25,26)$. The insulin-induced regulation of PGC1 $\alpha$ and SREBP-1c mRNA expression has been shown to be impaired in the skeletal muscle of T2DM patients $(14,25)$. 
There are indications that weight loss is able to improve partly the switch between fat and carbohydrate oxidation in the postprandial state in obese or IGT subjects $(5,27)$, which may be related to the effect of insulin on genes involved in lipid metabolism. Surgically induced weight loss increased PGC1 $\alpha$ (mRNA expression) in previous morbidly obese subjects $(28,29)$, whilst Berggren et al. observed no change in PGC1 $\alpha$ expression after weight loss in obese subjects (30).

The current study was undertaken to compare the expression levels of candidate genes involved in lipid metabolism in muscle biopsies of obese NGT and IGT subjects during fasting or insulin mediated conditions. Secondly, the impact of weight loss on fatty acid metabolism-related gene expression was studied in the obese IGT subjects.

\section{Methods}

\section{Subjects}

Five obese NGT men and seven obese IGT men, comparable for age and BMI, participated in the study. Glucose tolerance was measured with a standard oral glucose tolerance test with capillary plasma sampling at baseline and after two hours according to the World Health Organization (WHO) guidelines. Additional inclusion criteria were obesity (Body Mass Index $(\mathrm{BMI})>30 \mathrm{~kg} / \mathrm{m}^{2}$ ), diastolic blood pressure $<100 \mathrm{mmHg}$, no major organ dysfunction and no use of lipid and glucose lowering medication. The NGT men had no family history of diabetes. Subjects were not involved in any organized sports activities for more than 3 hours a week. The experimental protocol was approved by the local Medical Ethical Committee of the Maastricht University. All subjects were informed about the aims of the study and gave their written informed consent.

\section{Study design}

The NGT men and IGT men underwent measurements for body composition, aerobic capacity and insulin sensitivity. Body weight (BW) was determined on an electronic scale. Body composition (fat mass (FM) and fat free mass (FFM)) were determined by hydrostatic weighing with simultaneous correction for lung volume and body fat percentage was calculated according to the equation of Siri (31). Waist and hip circumference were measured to the nearest $1 \mathrm{~cm}$. Peak oxygen uptake $\left(\mathrm{VO}_{2} \mathrm{max}\right)$ was determined during an incremental exhaustive bicycle ergometer test. Insulin sensitivity was determined with a hyperinsulinemic euglycemic clamp as described below. Percutaneous needle biopsies were taken, at the beginning and at the end of the hyperinsulinemic clamp, from the vastus lateralis muscle under local anesthesia of the skin using the Bergström method with suction (Figure 1) (32). Biopsies were immediately frozen in liquid nitrogen. 
From five men of the IGT group, we obtained complete data on skeletal muscle gene expression before and after a 12-week weight loss program (Figure 1). During the first four weeks, the subjects were provided with a very low calorie diet (VLCD) (2 MJ/day) based on shakes (Modifast Nutrition et Santé, Breda, the Netherlands) containing all the essential macro- and micronutrients. From weeks five to eight, the shakes were gradually replaced by normal meals, increasing the energy content of the diet up to $4.2 \mathrm{MJ} /$ day. In the last four weeks, the subjects were kept in energy balance by prescribing detailed weekly menus. After at least 2 weeks of energy balance, the measurements as described above were repeated (weeks 10-12).

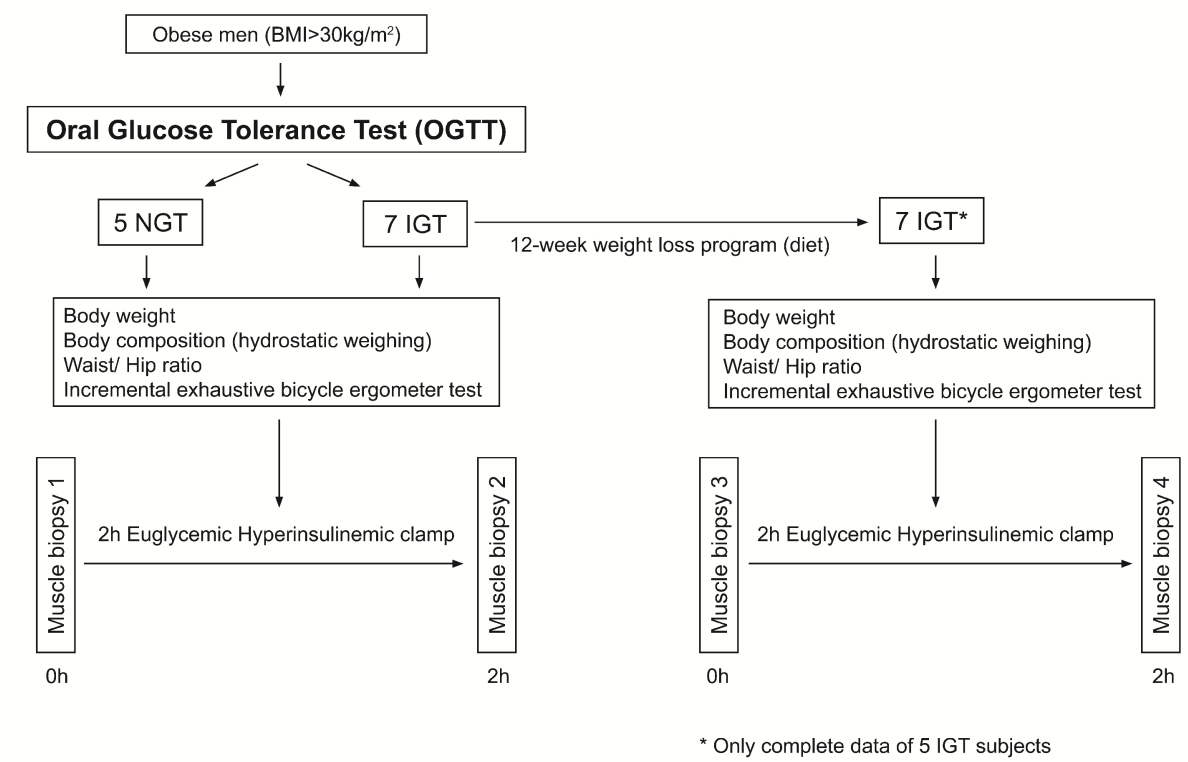

Figure 1. Scheme of the study design.

\section{Euglycemic-hyperinsulinemic clamp}

Subjects arrived at the laboratory by car or public transport after an overnight fast (10-12 hours). Insulin was infused at a constant rate $\left(1 \mathrm{mU}^{*} \mathrm{~kg} \mathrm{BW}^{-1 *} \mathrm{~min}^{-1}\right)$ with glucose clamped at $4.5 \pm 0.2 \mathrm{mmol} / \mathrm{l}$ with an intravenous infusion of $20 \% \mathrm{w} / \mathrm{v}$ glucose solution. Insulin sensitivity (M-value) was calculated as the glucose infusion rate per $\mathrm{kg} \mathrm{FFM}\left(\mu \mathrm{mol}^{\star} \mathrm{kgFFM}^{-1 *} \mathrm{~min}^{-1}\right)$ during a steady state of 30 minutes after at least 120 minutes of insulin infusion. 


\section{Biochemical analysis}

The muscle biopsies were homogenized in ice-cold Tris-EDTA buffer at pH 7.4. The homogenates were subsequently sonicated for $4 \times 15 \mathrm{~s}$ and were centrifuged at $10,000 \mathrm{~g}$ for $2 \mathrm{~min}$ at $4^{\circ} \mathrm{C}$ to remove cell debris. Citrate synthase (CS) was determined by the method of Sherpherd and Garland (33), whereas 3-hydroxyacylCoA dehydrogenase (HAD) was assayed according to Bergmeyer (34).

\section{Total RNA preparation}

Total RNA was extracted from frozen skeletal muscle specimens using TRIzol reagent (Invitrogen, Breda, the Netherlands) and purified with the RNeasy Mini Kit (Qiagen, Venlo, the Netherlands). RNA quantity was measured with the ND-100 spectrophotometer (Isogen Life Science, ljsselstein, the Netherlands), and RNA integrity was analysed on an Agilent 2100 BioAnalyzer (Agilent Technologies, Amsterdam, The Netherlands) using nanochips according to manufacturer's instructions. A RNA Integrity Number above 6 has been judged acceptable for this study.

\section{Quantification of messenger RNAs}

Real-time quantitative Polymerase Chain Reaction (PCR) was used to analyze RNA samples. Total RNA was reverse-transcribed in a $20 \mu$ reaction using the iScript cDNA synthesis Kit (Bio-Rad,Veenendaal, the Netherlands) according to the manufacturer's instructions. Primers were designed using Beacon designer or were obtained by using Primer Bank (Table 1). Real-time PCR was carried out in an icycler thermal cycler upgraded with an MylQ ${ }^{\mathrm{TM}}$ Single Color Real-Time PCR detection system (Bio-Rad, Veenendaal, the Netherlands). Gene expression levels were normalized using housekeeping genes Glyceraldehyde 3-phosphate dehydrogenase (GAPDH) and Ribosomal Protein L13a (RPL13a). GeNorm was used to test the stability of the housekeeping genes (35).

Table 1. Sequences of the primers used for mRNA quantitation by RT-PCR.

\begin{tabular}{lll}
\hline Gene & Sense Primer & Antisense Primer \\
\hline GAPDH & GAGTCAACGGATTTGGTCGT & TTGATTTTGGAGGGATCTCG \\
RPL13a & CCTGGAGGAGAAGAGGAAAGAGA & TTGAGGACCTCTGTGTATTTGTCAA \\
PGC1 $\alpha$ & CCAGGTCAAGATCAAGGTCTCCAG & TTCGGTGCGTGCGGTGTC \\
PPAR $\alpha$ & AAAAGCCTAAGGAACCGTTCTG & TATCGTCCGGGTGGTTGCT \\
SREBP-1C & ACACAGCAACCAGAAACTCAAGC & GCCGACACCAGATCCTTCAGAG \\
SREBP-2 & GCACCACTCCGCAGACGAG & TGGACTTGAGGCTGAAGGACTTG \\
ChREBP & GCCTCAAGGTGAGCAAAGCTA & GCTGGCACAGGTTAATGGC \\
ACC-2 & GCAAGAACGTGTGGGGTTACT & TCGCCTCGGATGGACAGTT \\
\hline
\end{tabular}




\section{Statistical analysis}

Using our sample size of 5 NGT subjects and 7 IGT subjects, our study had enough power $(\beta=0.8, \alpha=0.05)$ to detect an effect size of 1.82 as normalized expression between the NGT and IGT group for all genes. Using our sample size of 5 IGT subjects, our study had enough power $(\beta=0.8, \alpha=0.05)$ to detect an effect size of 1.68 as normalized expression in IGT subjects before compared to after weight loss for all genes. Power calculation was performed using $G^{*}$ Power 1.3 (Institute for Experimental Psychology, Heinrich-Heine University Düsseldorf). Differences between NGT $(n=5)$ and IGT men $(n=7)$ were statistically examined by two-tailed Student's t-test for unpaired samples or over time by ANOVA (repeated measures). IGT men before $(n=5)$ and after weight loss $(n=5)$ were compared with a two-tailed Student's t-test for paired samples. Results are given as mean \pm SEM. A p-value $<0.05$ was considered as statistically significant. Statistical analysis was performed using SPSS 16.0 for Macintosh.

\section{Results}

\section{Subject characteristics}

The IGT and NGT men were comparable for age and BMI. They were also comparable with respect to body composition and peak oxygen uptake (Table 2). The IGT men tended to be more insulin resistant than NGT men ( $p=0.098$, Table 3A), had slightly elevated fasting glucose concentrations (not significant) and had by definition increased $2 \mathrm{~h}$ glucose concentrations.

The dietary intervention induced a $15.5 \pm 4.3 \mathrm{~kg}$ reduction in body weight, which could be attributed to $20 \%$ by loss of FFM and by $80 \%$ by loss of FM (Table 2). After weight loss, there was a significant increase in insulin sensitivity $(p=0.02)$, (Table 3B). Skeletal muscle CS activity $(p=0.08)$ tended to increase after weight loss, whilst the HAD activity did not change (Table 2). The peak oxygen uptake did not change after weight loss (Table 2). 
Table 2. Clinical characteristics of study subjects.

\begin{tabular}{|c|c|c|c|c|c|c|}
\hline & \multirow[b]{2}{*}{ NGT $(n=5)$} & \multirow[b]{2}{*}{ IGT (n=7) } & \multirow[b]{2}{*}{$\begin{array}{l}\mathrm{P} \text { - } \\
\text { value }\end{array}$} & \multicolumn{2}{|c|}{ IGT } & \multirow[b]{2}{*}{$\begin{array}{c}\text { P- } \\
\text { value }\end{array}$} \\
\hline & & & & $\begin{array}{c}\text { before WL } \\
(n=5)\end{array}$ & $\begin{array}{c}\text { after WL } \\
(n=5)\end{array}$ & \\
\hline Age (years) & $59.8 \pm 1.9$ & $57.1 \pm 3.5$ & 0.57 & & & \\
\hline Body weight (kg) & $99.8 \pm 6.5$ & $92.9 \pm 3.4$ & 0.33 & $95.5 \pm 4.0$ & $80.0 \pm 3.7$ & 0.001 \\
\hline $\mathrm{BMI}\left(\mathrm{kg} / \mathrm{m}^{2}\right)$ & $33.8 \pm 1.7$ & $32.2 \pm 0.7$ & 0.34 & $32.3 \pm 0.6$ & $27.0 \pm 0.8$ & 0.001 \\
\hline Body fat (\%) & $35.9 \pm 2.2$ & $32.5 \pm 1.3$ & 0.18 & $34.0 \pm 1.1$ & $25.0 \pm 1.8$ & 0.002 \\
\hline Fat free mass $(\mathrm{kg})$ & $63.6 \pm 3.3$ & $62.6 \pm 1.3$ & 0.75 & $62.9 \pm 1.7$ & $59.7 \pm 1.9$ & 0.04 \\
\hline Fat mass (kg) & $36.2 \pm 4.2$ & $30.5 \pm 2.2$ & 0.23 & $32.6 \pm 2.3$ & $20.2 \pm 2.1$ & 0.001 \\
\hline Waist-hip ratio & $1.02 \pm 0.03$ & $1.03 \pm 0.01$ & 0.62 & $1.05 \pm 0.01$ & $0.98 \pm 0.01$ & 0.01 \\
\hline $\begin{array}{l}\text { VO2max } \\
\left(\mathrm{ml} \mathrm{O}_{2}{ }^{*} \mathrm{~kg} \mathrm{FFM}^{-1 *} \mathrm{~min}^{-1}\right)\end{array}$ & $40.3 \pm 2.7$ & $39.5 \pm 1.8$ & 0.82 & $41.4 \pm 2.2$ & $42.6 \pm 3.8$ & 0.96 \\
\hline $\mathrm{FPG}(\mathrm{mmol} / \mathrm{l})$ & $5.8 \pm 0.3$ & $6.6 \pm 0.3$ & 0.12 & & & \\
\hline 2h PG (mmol/l) & $7.5 \pm 0.5$ & $11.7 \pm 0.7$ & 0.001 & & & \\
\hline CS (U/g wet weight) & $3.0 \pm 0.8$ & $4.2 \pm 0.4$ & 0.20 & $4.3 \pm 0.5$ & $7.4 \pm 1.2$ & 0.08 \\
\hline HAD (U/g wet weight) & $4.5 \pm 1.0$ & $5.0 \pm 0.5$ & 0.66 & $5.0 \pm 0.8$ & $3.7 \pm 1.0$ & 0.43 \\
\hline
\end{tabular}

Values are mean \pm SEM. Student's t-test for unpaired samples NGT vs. IGT, two-tailed. Student's t-test for paired samples IGT before weight loss vs. IGT after weight loss. NGT, normal glucose tolerance; IGT, impaired glucose tolerance; WL, weight loss; BMI, body mass index; FPG, fasting plasma glucose; 2hPG, 2 hour plasma glucose; CS, citrate synthase activity; HAD, 3-hydroxyacyl-CoA dehydrogenase.

Fasting glucose, insulin and free fatty acids (FFA) were not significantly different between IGT and NGT men (Table 3A). After weight loss, IGT men had a significantly lower insulin concentration during fasting $(p=0.03)$ and during steady state at the end of the clamp $(p=0.04)$. Furthermore, the FFA concentration tended to be lower in the IGT men after weight loss in the steady state period at the end of the clamp compared to before weight loss $(p=0.07)$ (Table 3B).

Table 3A. M-value and plasma metabolites during fasting and during the steady state of the euglycemic hyperinsulinemic clamp.

\begin{tabular}{|c|c|c|c|c|}
\hline & \multicolumn{2}{|c|}{ NGT $(n=5)$} & \multicolumn{2}{|c|}{ IGT $(n=7)$} \\
\hline & Fasting & SS & Fasting & ss \\
\hline Glucose (mmol/l) & $5.9 \pm 0.1$ & $4.5 \pm 0.1$ & $6.2 \pm 0.2$ & $4.4 \pm 0.1$ \\
\hline Insulin (mU/l) & $12.3 \pm 2.3$ & $106.8 \pm 6.9$ & $15.5 \pm 2.8$ & $94.5 \pm 7.2$ \\
\hline $\mathrm{FFA}(\mu \mathrm{mol} / \mathrm{l})$ & $629 \pm 120$ & $162 \pm 28$ & $652 \pm 139$ & $149 \pm 19$ \\
\hline $\begin{array}{l}\text { Insulin sensitivity } \\
\left(\mathrm{M} \text {-value, } \mu \mathrm{mol}^{*} \mathrm{~kg} \mathrm{FFM}^{-1}{ }^{*} \mathrm{~min}^{-1}\right)\end{array}$ & n.a. & $34.2 \pm 6.7$ & n.a. & $20.8 \pm 4.1^{\#}$ \\
\hline
\end{tabular}

Values are mean \pm SEM. Student's t-test for unpaired samples NGT vs. IGT, two-tailed; \# $p<0.10$; NGT, normal glucose tolerance; IGT, impaired glucose tolerance; SS, steady state at the end of the clamp; FFA, free fatty acids. 
Table 3B. M-value and plasma metabolites during fasting and during the steady state of the euglycemic hyperinsulinemic clamp.

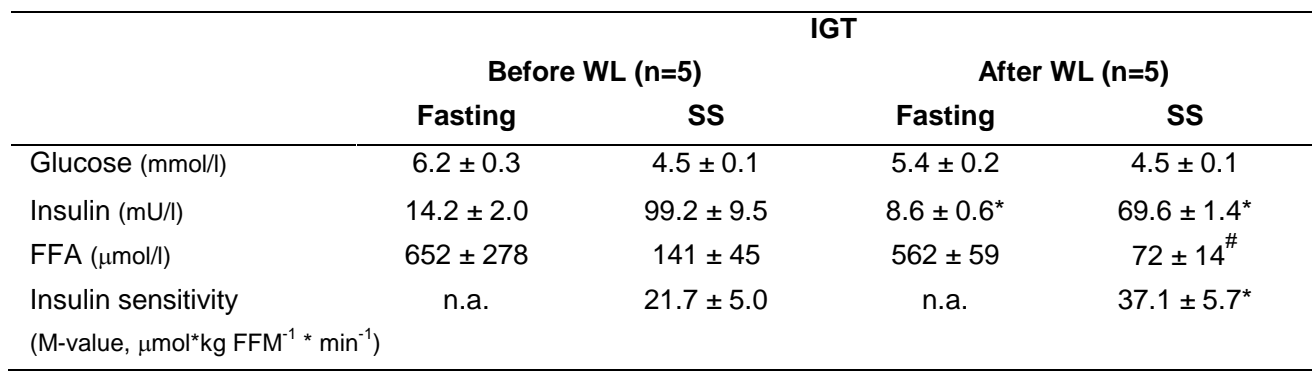

Values are mean \pm SEM. Student's t-test for paired samples IGT before weight loss vs. IGT after weight loss; * $p<$ 0.05 , \# $p<0.10$. IGT, impaired glucose tolerance; WL, weight loss; SS, steady state at the end of the clamp; FFA, free fatty acids.

\section{Skeletal muscle gene expression during fasting and during a euglycemic hyperinsulinemic clamp}

To determine fasting gene expression and the effect of insulin on skeletal muscle gene expression, six genes involved in fatty acid partitioning and oxidation were investigated. Fasting gene expression was not significantly different between the NGT and IGT men for all the genes tested. After the hyperinsulinemic clamp there was an overall upregulation of PGC1 $\alpha(p=0.003)$, SREBP-1c $(p=0.005)$, SREBP$2(p=0.005)$ and ACC-2 $(p=0.03)$ (Figures 2A, C, D and F). For SREBP-2, the upregulation was more pronounced in IGT vs. NGT men $(\Delta 0.23 \pm 0.15$ vs. $\Delta 0.07 \pm$ 0.18 respectively, $P=0.049$ ) (Figures $2 D$ and 3 ). $2 h$-insulin infusion did not affect PPAR $\alpha$ and ChREBP expression (Figures 2B and E).

$\square$ Before Clamp

After Clamp
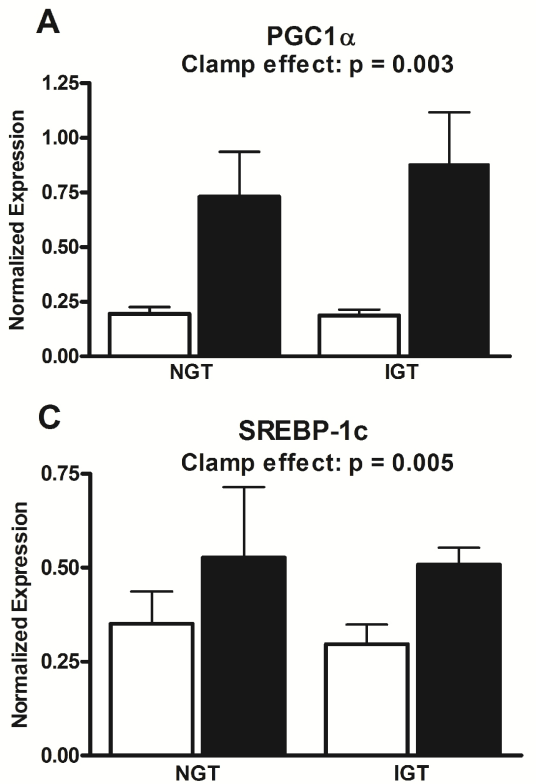

B

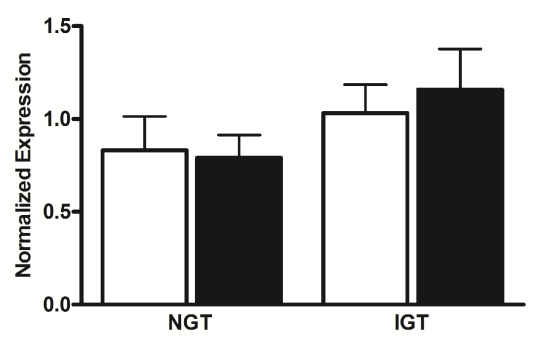

D

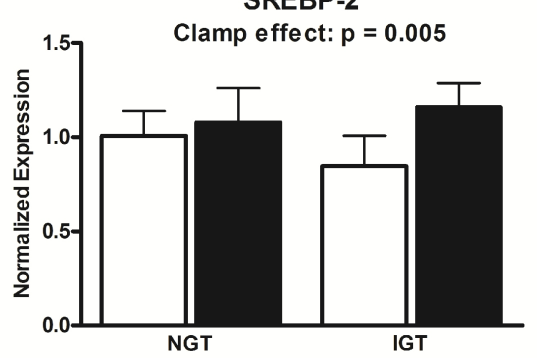


$\mathrm{E}$

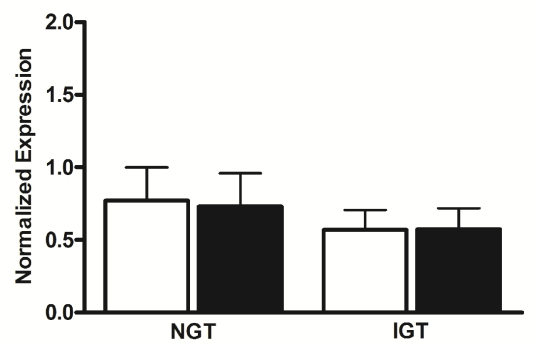

$\mathbf{F}$

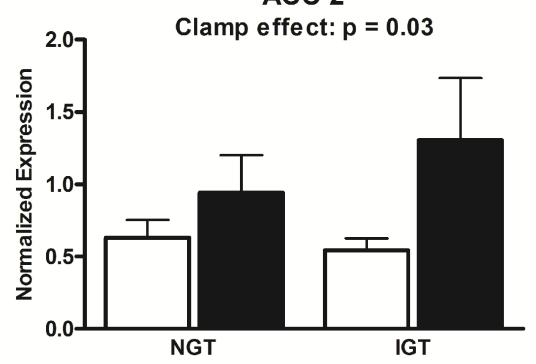

Figure 2. Effect of insulin on skeletal muscle gene expression. The mRNA levels of the various genes were determined by quantitative real-time PCR in skeletal muscle biopsies of NGT ( $n=5)$ and IGT $(\mathrm{n}=7)$ subjects before and after a $2 \mathrm{~h}$ euglycemic-hyperinsulinemic clamp. Results are presented as means \pm SEM.

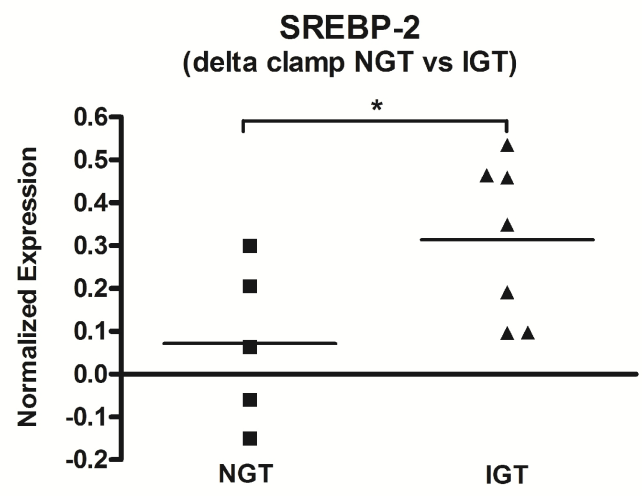

Figure 3. The change in SREBP-2 expression in NGT vs. IGT men induced by a $2 \mathrm{~h}$ euglycemichyperinsulinemic clamp. ${ }^{*} \mathrm{P}<0.05$ significantly different between NGT and IGT men. Data are presented as values after clamp minus values before clamp.

\section{Effect of weight loss on skeletal muscle gene expression}

We investigated in IGT men whether weight loss could induce changes in mRNA level of the six target genes. Although the IGT men had improved insulin sensitivity after weight loss $(p=0.02)$, this effect could not be detected on a transcriptional level (Figure 4). Both PGC1 $\alpha$ and PPAR $\alpha$, genes thought to be involved in skeletal muscle fat oxidation, showed no increased expression after weight loss (Figures 4A and B). There was also no effect of weight loss on the expression of SREBP1c, SREBP-2, ChREBP and ACC-2 (Figures 4C-F). 
A

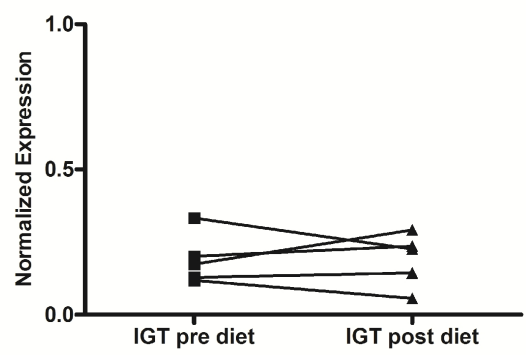

C

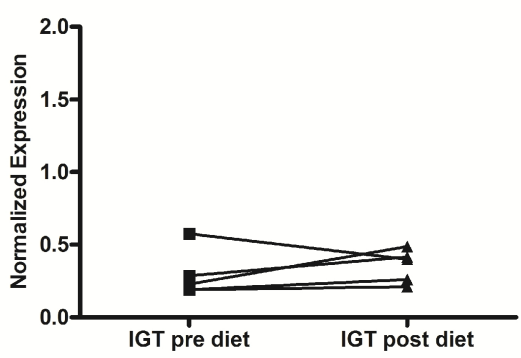

E

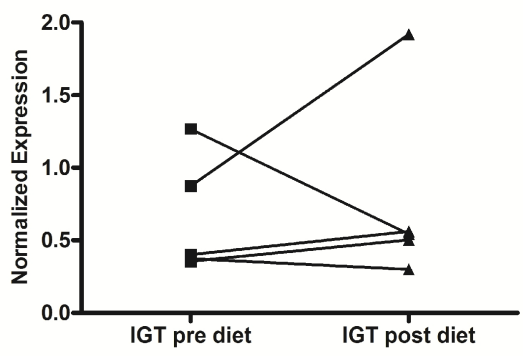

B

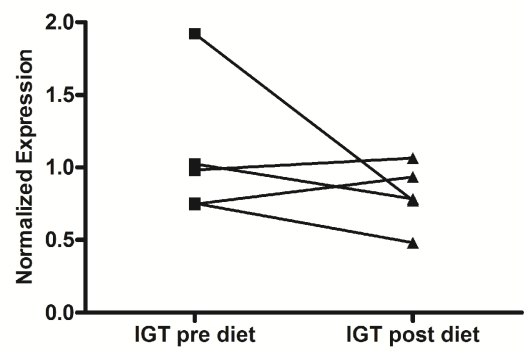

D

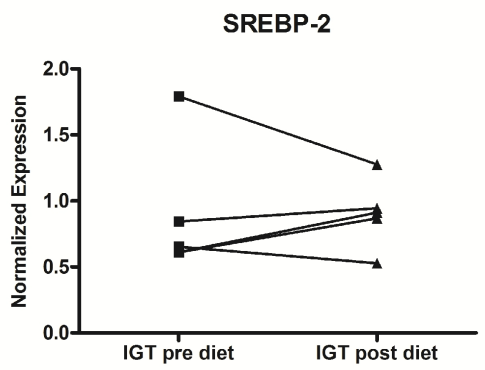

$\mathbf{F}$

ACC-2

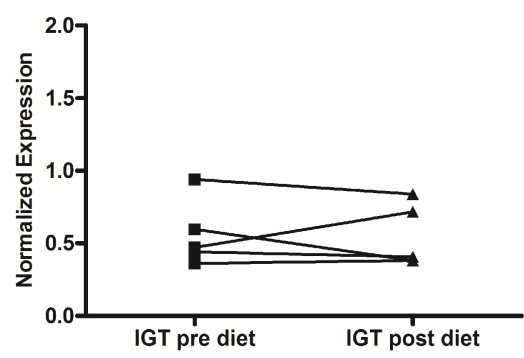

Figure 4. Skeletal muscle gene expression in IGT men before and after weight loss. The mRNA levels of the various genes were determined by quantitative real-time PCR in skeletal muscle biopsies of IGT ( $n=5)$ subjects before and after weight loss. Paired Student's t-test, two-tailed.

\section{Discussion}

In the present study, we have demonstrated that SREBP-2 may play an important role in skeletal muscle fatty acid handling since it was differentially expressed between NGT and IGT men after insulin infusion. The basal level of SREBP-2 in 
the IGT subjects was lower than in the NGT subjects, although not significantly different. An infusion of insulin in the IGT men, acutely upregulated SREBP-2 expression to levels comparable in NGT subjects. The lower baseline expression could have contributed to the more pronounced increase of SREBP-2 expression in IGT men compared with NGT men. SREBP-2 is a member of the SREBP family, a group of membrane-bound transcription factors, which are synthesized as inactive precursor proteins. They undergo posttranslational cleavage in order to enter the nucleus and stimulate transcription of numerous target genes (36). In liver, SREBP-2 mainly activates genes involved in cholesterol synthesis (18). The exact role of SREBP-2 in skeletal muscle metabolism remains to be elucidated. Recent studies have demonstrated that SREBPs are central regulators of membrane lipid biosynthesis $(16,37)$. Mahoney et al. report an upregulation of SREBP-2 in skeletal muscle after eccentric exercise (38). They suggest that SREBP-2 may activate a transcriptional program for de novo membrane synthesis since eccentric exercise can induce membrane damage. Based on these findings, it can be speculated that in our study SREBP-2 is more upregulated in IGT men after insulin infusion to repair the mitochondrial membrane damage possibly induced by lipid peroxidation. However, the present study has no data to address this hypothesis. From literature, it is known that IGT men suffer from oxidative stress, defined as a persistent imbalance between the production of highly reactive oxygen/nitrogen species (39). Mitochondria are a major source of reactive oxygen species. Accumulation of fatty acids in the vicinity of the mitochondrial matrix makes them prone to lipid peroxidation, which eventually may result in damaged mitochondrial proteins and reduced oxidative capacity $(40,41)$. SREBP-2 showed altered insulin responsiveness in IGT men compared with NGT men and may thus be an interesting candidate for further research to elucidate its exact role in skeletal muscle.

Besides SREBP-2, several other genes were upregulated after the euglycemichyperinsulinemic clamp, including PGC1 $\alpha$, SREBP-1C and ACC-2. Our data are consistent with Ling et al. who found that insulin increased the mRNA level of PGC1 $\alpha$ in muscle biopsies from healthy young and elderly twins before and after a clamp (42). This increase can mediate the expression of GLUT-4 in large part by binding to and coactivating the muscle-selective transcription factor myocytespecific enhancer factor $2 \mathrm{C}$ (MEF2C) $(43,44)$. Many groups have established that insulin upregulates SREBP-1c mRNA and mature SREBP-1 protein and is accompanied by increases in FA biosynthetic gene expression $(17,25)$. ACC-2 is one of these genes that catalyzes the synthesis of malonyl-CoA from acetyl-CoA. Increased levels of malonyl-CoA lead to the inhibition of CPT-1 and, subsequently, to the inhibition of mitochondrial fatty acid oxidation $(45,46)$. Our results, indeed, show an upregulation of SREBP-1C and, subsequently, an upregulation of ACC-2 after insulin stimulation. 
Previous studies indicated that the transcriptional profile in the insulin resistant muscle might be more directed towards fat storage than oxidation. Although Morino et al. have reported similar gene expression levels in insulin resistant and healthy controls (47), other studies indicate a reduced expression of PGC1 $\alpha$ and oxidative genes as early factors in the etiology of $\operatorname{T2DM}(14,23,24,48)$. Moreover, an upregulation of ACC-2 has been observed in T2DM patients compared with lean subjects (14). This could not be confirmed in the present study, which did not show a decreased expression of PGC1 $\alpha$ and an increased expression of ACC-2 in IGT men compared to NGT men in the fasted state. Although we cannot exclude that we missed small differences between groups (effect size smaller than 1.82 as normalized expression), this does not explain most discrepancies between our data and several previous studies. Possibly, differences in the degree of insulin resistance between the subject groups of the individual studies may have played a role. In most studies, more extreme phenotypes are compared. In our study, differences in insulin resistance were less extreme and subjects were comparable for obesity, which may explain the absence of relevant numerical differences in baseline gene expression that were observed.

The second objective of the present work was to define whether weight loss could have an impact on the expression of genes involved in FA handling. Weight loss significantly increased insulin sensitivity (by $71 \%$ ) and tended to improve muscle oxidative capacity as evidenced by CS activity. Nevertheless, our data do not support an improvement on transcriptional level of the genes examined here. This is consistent with Simoneau et al. who also did not detect changes in several different markers of skeletal muscle FA metabolism in obese NGT men after a weight loss intervention based on a VLCD (11), but not with all other studies showing an upregulation of genes involved in lipid oxidation $(28,29,49)$. This may be explained by the finding that no apparent disturbances at basal transcriptional level were present between NGT and IGT men and that, possibly, the expression of these genes is not regulated by obesity per se. Small differences after weight loss may have been missed because of the relatively small sample size, and the results will need confirmation from other studies.

In conclusion, insulin could acutely increase the expression of PGC1 $\alpha$, SREBP-1C, SREBP-2 and ACC-2 in obese subjects. Accumulating data indicate that defects in insulin-regulated gene expression may be involved in the etiology of insulin resistance and T2DM. In the present work, SREBP-2 was identified as a potential novel candidate because of its altered insulin responsiveness in IGT men compared with NGT men. 


\section{Acknowledgements}

In the first place we want to thank the volunteers for their time and effort to make this study possible. We thank Jos Stegen, Joan Senden, Marja van der HulstOckeloen and Tanja Hermans for their excellent analytical and dietary support. This study was supported by grants from the Dutch Diabetes Research Foundation (DFN 98.901 and DFN 2000.00.020) and in part by the Top Institute Food and Nutrition, with financial support by the Dutch government. 


\section{References}

1. Wild S, Roglic G, Green A, Sicree R, King H. Global prevalence of diabetes: estimates for the year 2000 and projections for 2030. Diabetes care 2004;27(5):1047-53.

2. Saad MF, Knowler WC, Pettitt DJ, Nelson RG, Charles MA, Bennett PH. A two-step model for development of non-insulin-dependent diabetes. The American journal of medicine 1991;90(2):229-35.

3. Weyer C, Tataranni PA, Bogardus C, Pratley RE. Insulin resistance and insulin secretory dysfunction are independent predictors of worsening of glucose tolerance during each stage of type 2 diabetes development. Diabetes care 2001;24(1):89-94.

4. Blaak EE, Wagenmakers AJ. The fate of [U-(13)C]palmitate extracted by skeletal muscle in subjects with type 2 diabetes and control subjects. Diabetes 2002;51(3):784-9.

5. Corpeleijn E, Mensink M, Kooi ME, Roekaerts PM, Saris WH, Blaak EE. Impaired skeletal muscle substrate oxidation in glucose-intolerant men improves after weight loss. Obesity (Silver Spring, Md 2008;16(5):1025-32.

6. Hegarty BD, Furler SM, Ye J, Cooney GJ, Kraegen EW. The role of intramuscular lipid in insulin resistance. Acta physiologica Scandinavica 2003;178(4):373-83.

7. Ellis BA, Poynten A, Lowy AJ, et al. Long-chain acyl-CoA esters as indicators of lipid metabolism and insulin sensitivity in rat and human muscle. American journal of physiology 2000;279(3):E554-60.

8. Itani SI, Ruderman NB, Schmieder F, Boden G. Lipid-induced insulin resistance in human muscle is associated with changes in diacylglycerol, protein kinase C, and IkappaB-alpha. Diabetes 2002;51(7):2005-11.

9. Holland WL, Brozinick JT, Wang LP, et al. Inhibition of ceramide synthesis ameliorates glucocorticoid-, saturated-fat-, and obesity-induced insulin resistance. Cell metabolism 2007;5(3):167-79

10. Corpeleijn E, Saris WH, Blaak EE. Metabolic flexibility in the development of insulin resistance and type 2 diabetes: effects of lifestyle. Obesity Reviews 2009;10(2):178-93.

11. Simoneau JA, Veerkamp JH, Turcotte LP, Kelley DE. Markers of capacity to utilize fatty acids in human skeletal muscle: relation to insulin resistance and obesity and effects of weight loss. Faseb Journal 1999;13(14):2051-60.

12. Blaak EE, Wagenmakers AJ, Glatz JF, et al. Plasma FFA utilization and fatty acid-binding protein content are diminished in type 2 diabetic muscle. American journal of physiology 2000;279(1):E146-54.

13. Ruderman NB, Saha AK, Vavvas D, Witters LA. Malonyl-CoA, fuel sensing, and insulin resistance. The American journal of physiology 1999;276(1 Pt 1):E1-E18.

14. Debard C, Laville M, Berbe V, et al. Expression of key genes of fatty acid oxidation, including adiponectin receptors, in skeletal muscle of Type 2 diabetic patients. Diabetologia 2004;47(5):917-25.

15. Mensink M, Blaak EE, Vidal H, De Bruin TW, Glatz JF, Saris WH. Lifestyle changes and lipid metabolism gene expression and protein content in skeletal muscle of subjects with impaired glucose tolerance. Diabetologia 2003;46(8):1082-9.

16. Porstmann T, Griffiths B, Chung YL, et al. PKB/Akt induces transcription of enzymes involved in cholesterol and fatty acid biosynthesis via activation of SREBP. Oncogene 2005;24(43):6465-81.

17. Sewter C, Berger D, Considine RV, et al. Human obesity and type 2 diabetes are associated with alterations in SREBP1 isoform expression that are reproduced ex vivo by tumor necrosis factor-alpha. Diabetes 2002;51(4):1035-41.

18. Horton JD, Shimomura I, Brown MS, Hammer RE, Goldstein JL, Shimano H. Activation of cholesterol synthesis in preference to fatty acid synthesis in liver and adipose tissue of transgenic mice overproducing sterol regulatory element-binding protein-2. The Journal of clinical investigation 1998;101(11):2331-9.

19. Phielix E, Schrauwen-Hinderling VB, Mensink M, et al. Lower intrinsic ADP-stimulated mitochondrial respiration underlies in vivo mitochondrial dysfunction in muscle of male type 2 diabetic patients. Diabetes 2008;57(11):2943-9.

20. Petersen KF, Dufour S, Befroy D, Garcia R, Shulman GI. Impaired mitochondrial activity in the insulin-resistant offspring of patients with type 2 diabetes. The New England journal of medicine 2004;350(7):664-71. 
21. Boushel R, Gnaiger E, Schjerling P, Skovbro M, Kraunsoe R, Dela F. Patients with type 2 diabetes have normal mitochondrial function in skeletal muscle. Diabetologia 2007;50(4):7906.

22. De Feyter HM, van den Broek NM, Praet SF, Nicolay K, van Loon LJ, Prompers JJ. Early or advanced stage type 2 diabetes is not accompanied by in vivo skeletal muscle mitochondrial dysfunction. European journal of endocrinology / European Federation of Endocrine Societies 2008;158(5):643-53.

23. Mootha VK, Lindgren CM, Eriksson KF, et al. PGC-1alpha-responsive genes involved in oxidative phosphorylation are coordinately downregulated in human diabetes. Nature genetics 2003;34(3):267-73.

24. Patti ME, Butte AJ, Crunkhorn S, et al. Coordinated reduction of genes of oxidative metabolism in humans with insulin resistance and diabetes: Potential role of PGC1 and NRF1. Proceedings of the National Academy of Sciences of the United States of America 2003;100(14):8466-71.

25. Ducluzeau PH, Perretti N, Laville M, et al. Regulation by insulin of gene expression in human skeletal muscle and adipose tissue. Evidence for specific defects in type 2 diabetes. Diabetes 2001;50(5):1134-42.

26. O'Brien RM, Granner DK. Regulation of gene expression by insulin. Physiological reviews 1996;76(4):1109-61.

27. Kelley DE, Goodpaster B, Wing RR, Simoneau JA. Skeletal muscle fatty acid metabolism in association with insulin resistance, obesity, and weight loss. The American journal of physiology 1999;277(6 Pt 1):E1130-41.

28. Hernandez-Alvarez MI, Chiellini C, Manco M, et al. Genes involved in mitochondrial biogenesis/function are induced in response to bilio-pancreatic diversion in morbidly obese individuals with normal glucose tolerance but not in type 2 diabetic patients. Diabetologia 2009;52(8):1618-27.

29. Gastaldi G, Russell A, Golay A, et al. Upregulation of peroxisome proliferator-activated receptor gamma coactivator gene (PGC1A) during weight loss is related to insulin sensitivity but not to energy expenditure. Diabetologia 2007;50(11):2348-55.

30. Berggren JR, Boyle KE, Chapman WH, Houmard JA. Skeletal muscle lipid oxidation and obesity: influence of weight loss and exercise. American journal of physiology 2008;294(4):E726-32.

31. Siri WE. The gross composition of the body. Advances in biological and medical physics 1956;4:239-80.

32. Bergstrom J, Hermansen L, Hultman E, Saltin B. Diet, muscle glycogen and physical performance. Acta physiologica Scandinavica 1967;71(2):140-50.

33. Shepherd D GP. Citrate synthase from the rat liver. Methods Enzymology 1969;13:11-9.

34. Bergmeyer H. 3-Hydroxyacyl CoA dehydrogenase. Lactate dehydrogenase. New York: Academic, 1974.

35. Vandesompele J, De Preter K, Pattyn F, et al. Accurate normalization of real-time quantitative RT-PCR data by geometric averaging of multiple internal control genes. Genome biology 2002;3(7):RESEARCH0034.

36. Brown MS, Goldstein JL. The SREBP pathway: regulation of cholesterol metabolism by proteolysis of a membrane-bound transcription factor. Cell 1997;89(3):331-40.

37. Radhakrishnan A, Goldstein JL, McDonald JG, Brown MS. Switch-like control of SREBP-2 transport triggered by small changes in ER cholesterol: a delicate balance. Cell metabolism 2008;8(6):512-21.

38. Mahoney DJ, Safdar A, Parise G, et al. Gene expression profiling in human skeletal muscle during recovery from eccentric exercise. American Journal of Physiology - Regulatory, Integrative and Comparative Physiology 2008;294(6):R1901-10.

39. Houstis N, Rosen ED, Lander ES. Reactive oxygen species have a causal role in multiple forms of insulin resistance. Nature 2006;440(7086):944-8.

40. Fridlyand LE, Philipson LH. Reactive species and early manifestation of insulin resistance in type 2 diabetes. Diabetes, obesity \& metabolism 2006;8(2):136-45.

41. Schrauwen P, Hesselink MK. Oxidative capacity, lipotoxicity, and mitochondrial damage in type 2 diabetes. Diabetes 2004;53(6):1412-7.

42. Ling $C$, Poulsen $P$, Carlsson E, et al. Multiple environmental and genetic factors influence skeletal muscle PGC-1alpha and PGC-1beta gene expression in twins. The Journal of clinical investigation 2004;114(10):1518-26.

43. Michael LF, Wu Z, Cheatham RB, et al. Restoration of insulin-sensitive glucose transporter (GLUT4) gene expression in muscle cells by the transcriptional coactivator PGC-1. 
Proceedings of the National Academy of Sciences of the United States of America 2001;98(7):3820-5.

44. Benton CR, Nickerson JG, Lally J, et al. Modest PGC-1alpha overexpression in muscle in vivo is sufficient to increase insulin sensitivity and palmitate oxidation in subsarcolemmal, not intermyofibrillar, mitochondria. The Journal of biological chemistry 2008;283(7):4228-40.

45. Pender C, Trentadue AR, Pories WJ, Dohm GL, Houmard JA, Youngren JF. Expression of genes regulating malonyl-CoA in human skeletal muscle. Journal of cellular biochemistry 2006;99(3):860-7.

46. Rosa G, Manco M, Vega N, et al. Decreased muscle acetyl-coenzyme A carboxylase 2 mRNA and insulin resistance in formerly obese subjects. Obesity research 2003;11(11):130612.

47. Morino K, Petersen KF, Dufour S, et al. Reduced mitochondrial density and increased IRS-1 serine phosphorylation in muscle of insulin-resistant offspring of type 2 diabetic parents. The Journal of clinical investigation 2005;115(12):3587-93.

48. Heilbronn LK, Gan SK, Turner N, Campbell LV, Chisholm DJ. Markers of mitochondrial biogenesis and metabolism are lower in overweight and obese insulin-resistant subjects. The Journal of clinical endocrinology and metabolism 2007;92(4):1467-73.

49. Fabris R, Mingrone G, Milan G, et al. Further lowering of muscle lipid oxidative capacity in obese subjects after biliopancreatic diversion. The Journal of clinical endocrinology and metabolism 2004;89(4):1753-9. 


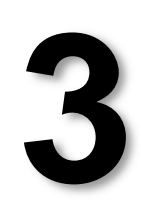

\title{
Calorie-restriction-like effects of 30 days resveratrol supplementation on energy metabolism and metabolic profile in obese humans
}

\author{
Silvie Timmers* \\ Ellen Konings* \\ Lena Bilet
}

Riekelt H. Houtkooper

Tineke van de Weijer Joris Hoeks

Sophie van der Krieken

Dongryeol Ryu

Esther Moonen-Kornips

Gijs H. Goossens

Matthijs K.C. Hesselink

Iris Kunz

Vera B. Schrauwen-Hinderling

Ellen E. Blaak Johan Auwerx

Patrick Schrauwen

* These authors contributed equally

Cell Metabolism 2011 Nov 2; 14(5):612-22. 


\section{Abstract}

Background: Resveratrol is a natural compound that affects energy metabolism and mitochondrial function and serves as a calorie restriction mimetic, at least in animal models of obesity.

Methods: Here we treated 11 healthy, obese men with placebo and $150 \mathrm{mg} /$ day resveratrol in a randomized double-blind cross-over study for 30 days.

Results: Resveratrol supplementation significantly reduced sleeping- and resting metabolic rate. In muscle, resveratrol activated AMPK, increased SIRT1 and PGC1 $\alpha$ protein levels, increased citrate synthase activity without change in mitochondrial content, and improved muscle mitochondrial respiration on a fatty acid-derived substrate. Furthermore, resveratrol elevated intramyocellular lipid levels, and decreased intrahepatic lipid content, circulating glucose, triglycerides, alanine-aminotransferase, and inflammation markers. Systolic blood pressure dropped and HOMA index improved after resveratrol. In the postprandial state, adipose tissue lipolysis and plasma fatty acid and glycerol decreased.

Conclusion: We demonstrate that 30 days of resveratrol supplementation induces profound metabolic changes in obese subjects, mimicking the effects of calorie restriction. 


\section{Introduction}

In our western society, the number of age-related chronic diseases such as obesity, diabetes and cancer increases progressively (1). The only nonpharmacological intervention known to date to alleviate these deleterious conditions is calorie restriction. Reduction of calorie intake to $30-50 \%$ below ad libitum levels, or every-other-day feeding, can delay the onset of age-related diseases, improve stress resistance, and decelerate functional decline (2-4). Although short-term dietary restriction has metabolic effects in humans such as lowering metabolic rate (5), improving insulin sensitivity $(6,7)$ and reducing cardiovascular risk factors (8), eating less for the sake of creating a desirable metabolic profile is unlikely to gain widespread compliance. As such, the focus has been on the development of calorie restriction mimics that evoke some of the benefits of calorie restriction without an actual reduction in calorie intake. In that respect, sirtuins are considered an important molecular target (9). Indeed, it was suggested that the yeast Sir2 gene (10) or its worm (11) and fly (12) orthologues are required for the effects of calorie restriction, although the relevance of the role of Sir2/SIRT1 as a strictu sensu longevity regulator is debated (13). What is clear, however, is that mammalian SIRT1 plays a context-dependent role in health span regulation, for instance by mediating effects in metabolic stress situations, such as high-fat diet-induced obesity (14-16). As such, SIRT1 confers protection against ageing-associated metabolic diseases such as glucose intolerance and cancer (1618). In light of the growing number of patients suffering from metabolic diseases, compounds that activate SIRT1 directly or indirectly, might offer protection against the onset of metabolic damage, and promote healthy ageing.

To this end, Howitz and colleagues performed an in vitro screen to identify small molecule activators of SIRT1 (19). Resveratrol, a natural polyphenolic compound present in various dietary components such as mulberries, peanuts, grapes and red wine, was identified as the most potent activator of SIRT1 (19). Recently, it was shown, however, that resveratrol may not activate SIRT1 directly $(20,21)$, but rather exerts its effects on SIRT1 through activation of AMPK $(14,22-26)$, although additional direct SIRT1 activation is not completely excluded (27). Regardless of the mode of activation, resveratrol treatment in mice fed a high calorie diet consistently improved various health parameters including glucose homeostasis, endurance and survival $(14-16,28)$, and has therefore been suggested to act as a calorie restriction mimetic. However, so far, no human studies have been reported that have systematically examined the metabolic effects of resveratrol in vivo.

Here, we gave obese subjects a dietary supplement containing $99 \%$ pure transresveratrol (resVida ${ }^{\mathrm{TM}}$ ), during 30 days and examined whole-body energy expenditure, substrate utilization, ectopic lipid storage, mitochondrial function, and 
lipolysis in adipose tissue and skeletal muscle using a combination of in vivo and ex vivo measurements. Our data show that like calorie restriction, resveratrol supplementation lowers energy expenditure, improves metabolic flexibility, as well as global health parameters.

\section{Methods}

The study protocol was reviewed and approved by the Medical Ethical Committee of Maastricht University Medical Centre (MUMC+). All study participants gave written informed consent before initiation of the study.

\section{Subject characteristics}

Eleven healthy, obese, male volunteers without family history of diabetes or any other endocrine disorder participated in this study (Table 1). None of the subjects were on medication or were engaged in sports activities for more than two hours per week. Body composition was determined by a dual-energy X-ray absorptiometry scan (DXA, Discovery A; Hologic Corp, Bedford, MA) and maximal aerobic capacity was measured as described (29).

Table 1. Subjects baseline characteristics.

\begin{tabular}{|c|c|c|c|}
\hline & Placebo & Resveratrol & P-value \\
\hline Age (years) & $52.5 \pm 2.1$ & $52.5 \pm 2.1$ & - \\
\hline Body weight (kg) & $100.1 \pm 3.5$ & $99.6 \pm 3.7$ & 0.50 \\
\hline BMI $\left(\mathbf{k g} / \mathbf{m}^{2}\right)$ & $31.59 \pm 0.74$ & $31.45 \pm 0.82$ & 0.48 \\
\hline Fat $(\%)$ & $26.44 \pm 0.53$ & $26.44 \pm 0.53$ & - \\
\hline $\mathrm{VO}_{2 \max }\left(\mathrm{ml} \cdot \mathrm{kg}^{-1} \cdot \mathrm{min}^{-1}\right)$ & $24.96 \pm 1.30$ & $24.80 \pm 1.00$ & 0.83 \\
\hline Systolic blood pressure (mmHg) & $131 \pm 3.1$ & $132 \pm 3.0$ & 0.22 \\
\hline Diastolic blood pressure $(\mathrm{mmHg})$ & $82 \pm 2.5$ & $83 \pm 2.6$ & 0.20 \\
\hline Glucose (mmol/l) & $5.44 \pm 0.10$ & $5.44 \pm 0.13$ & 0.96 \\
\hline Insulin (mU/I) & $16.37 \pm 1.76$ & $15.38 \pm 2.05$ & 0.67 \\
\hline Triglycerides (mmol/l) & $1.86 \pm 0.19$ & $1.92 \pm 0.21$ & 0.80 \\
\hline Non-esterified fatty acids ( $\mu \mathrm{mol} / \mathrm{l})$ & $357 \pm 69$ & $320 \pm 31$ & 0.56 \\
\hline
\end{tabular}

Subject characteristics at the start of the intervention (day 0$)$. Values are given as means \pm SEM $(n=11)$.

\section{Clinical study design}

Subjects participated in two experimental trials: a placebo and a resVida ${ }^{\mathrm{TM}}$ (150mg/day trans-resveratrol (99.9\%)) (provided by DSM Nutritional Products, Ltd) condition, in a randomized double-blind crossover design with a four-week washout period. Subjects were instructed to take the first supplement on the day after baseline measurements (d1) and the last supplement in the evening on day 29. The subjects were instructed to abstain from alcoholic beverages and foods 
containing substantial amounts of resveratrol (e.g. wine, red grapes, peanuts and berries) and were advised not to take any other food supplements during the study period. Compliance with these instructions was confirmed by verbal declaration of the subjects. Subjects were advised to maintain their normal living-, activity-, and sleeping-pattern during the intervention period. At the start (day 0) and end (day 30) of both intervention periods (resveratrol and placebo), blood samples were analyzed for general safety parameters including clinical chemistry, hematology and coagulation values. A twelve lead electrocardiogram (ECG) (Laméris, Veenendaal, The Netherlands) was performed at the beginning and end of both the resveratrol and placebo intervention. Each experimental trial lasted four weeks during which the subjects came on a weekly basis (day $0,7,14,21,30$ ) to the university. The weekly check-up took place in the morning after an overnight fast, and included a measurement of body mass and withdrawal of a small blood sample for the analysis of resveratrol (original and metabolites) to confirm compliance to the protocol. On day 28 in the evening, subjects came to the university for ${ }^{1} \mathrm{H}$-MRS measurements of the liver, to quantify intrahepatic lipid content, and post-exercise $\mathrm{PCr}$ recovery rate was examined by ${ }^{31} \mathrm{P}$-MRS to estimate in vivo mitochondrial function (30). To standardize food intake, subjects had lunch with the same food items in the two conditions and after lunch stayed fasted until the start of the measurement at $17 \mathrm{~h}$.

After the MRS measurements on day 28 , subjects stayed in the respiration chamber during $36 \mathrm{~h}$ to allow measurement of $24 \mathrm{~h}$ substrate oxidation and energy expenditure (31). Before their stay in the respiration chamber, a standardized evening meal was provided. In the respiration chamber subjects were fed in energy balance (2332.5 kcal/day, $13.1 \mathrm{~g}$ of protein, $54.0 \mathrm{~g}$ of carbohydrate, $32.9 \mathrm{~g}$ of fat) and followed an activity protocol as described (32). In the morning of day 30 , after subjects left the respiration chamber, a muscle biopsy was taken to investigate ex vivo mitochondrial respiration, which was followed by the withdrawal of a fasting blood sample for the analysis of the effect of resveratrol on circulating substrates. Hereafter, adipose tissue and skeletal muscle lipolysis was examined by means of microdialysis.

\section{Plasma biochemistry}

To check compliance, resveratrol metabolites were measured by mass spectrometry in plasma on day $0,7,14,21,29$ and 30 as described in the supplemental methods.

In the morning of day 30 -after a standardized overnight fast for 12 hours- and during the postprandial microdialysis test, blood samples were withdrawn for the determination of plasma metabolites according to standard procedures. Full experimental detail is described in the supplemental methods. 


\section{Blood pressure}

On day 0 and day 30, blood pressure was measured after an overnight fast. By placing an automatic inflatable cuff (Omron Healthcare, Hamburg, Germany) on the non-dominant arm in the resting condition, systolic and diastolic blood pressure was measured in triplicate. Mean systolic and diastolic blood pressure values were used to calculate the mean arterial pressure.

\section{Lipid accumulation in liver}

On day 28, before subjects underwent the respiration chamber measurement, proton magnetic resonance spectroscopy $\left({ }^{1} \mathrm{H}-\mathrm{MRS}\right)$ was used to quantify hepatic lipid content (IHL) on a $3 \mathrm{~T}$ whole body scanner (Achieva; Philips Healthcare, Best, The Netherlands) using a five-element coil as described (33), however with a repetition time $=4000 \mathrm{msec}$, echo time $=37 \mathrm{msec}$, and number of averages $=64$. To minimize motion artifacts, subjects were asked to breathe in the rhythm of the measurement and to be at end-expiration during acquisition of spectra. To determine the intensity of the lipid peak, the water signal was suppressed using frequency-selective prepulses. The unsuppressed water resonance was used as internal reference (number of averages $=32$ ) and spectra were fitted with AMARES (34) in the jMURI software (35). Values are given as T2-corrected ratios (according to (33)) of the $\mathrm{CH}_{2}$ peak, relative to the unsuppressed water resonance (as percentage).

\section{${ }^{31} \mathrm{P}$-MRS-based measurement of mitochondrial function}

On day $28,{ }^{31} \mathrm{P}$-MRS measurements were performed in vastus lateralis muscle on a $1.5 \mathrm{~T}$ whole-body scanner (Intera; Philips Health Care, Best, The Netherlands) essentially according to an established methodology (30).

\section{Muscle biopsy}

On day 30 , when subjects left the respiration chamber, a muscle biopsy was taken from the vastus lateralis muscle under local anesthesia (2\% lidocaine), as previously described (36). A portion of the muscle tissue was directly frozen in melting isopentane and stored at $-80^{\circ} \mathrm{C}$ until assayed. Another portion $(\sim 30 \mathrm{mg})$ was immediately placed in ice-cold preservation medium for determination of $e x$ vivo mitochondrial respiration (36).

\section{Molecular and protein expression}

Mitochondrial DNA copy number, gene expression by microarray, and protein expression by Western blot, were performed according to standard procedures as described in the supplemental methods. 


\section{High resolution respirometry}

Permeabilized muscle fibers were immediately prepared from the muscle tissue collected in the preservation medium, as described elsewhere $(36,37)$. Subsequently, the permeabilized muscle fibers ( 2.5 mg wet weight) were analyzed for mitochondrial function using an oxygraph (OROBOROS Instruments, Innsbruck, Austria) (38).

\section{Intramyocellular lipids}

Fresh cryosections $(5 \mu \mathrm{m})$ were stained for intramyocellular lipids (IMCL) by Oil Red $O$ staining combined with fibertyping and immunolabeling of the basal membrane marker laminin to allow quantification of IMCL, as described (39).

\section{Postprandial substrate utilization and tissue lipolysis}

In 10 subjects, the lipolytic effects of resveratrol in adipose tissue and skeletal muscle were successfully determined by microdialysis, essentially according to (40). A full description of the microdialysis method is provided as supplemental information.

\section{Statistical analysis}

Kolmogorov-Smirnov normality test was performed to evaluate normality distribution. Student's paired t-test was used to compare placebo and resVida supplementation in normally distributed data, otherwise Wilcoxon signed-rank test was used. For the microdialysis test day, postprandial area under the curve (AUC) of plasma and interstitial metabolites and indirect calorimetry data were calculated using the trapezium rule. In addition to the total AUC (Oh-6h after meal ingestion), also the early ( $0 h-2 h)$, mid ( $2 h-4 h)$ and late (4h-6h) AUCs were calculated to obtain more detailed information about the time course of postprandial responses. A PValue $<0.05$ was considered statistically significant. Data are reported as mean \pm SEM. Statistical analyses were performed using the statistical program SPSS 16.0 for Mac OS X.

\section{Results}

\section{Study design and plasma biochemistry}

Eleven obese but otherwise healthy male volunteers without a family history of diabetes or any other endocrine disorder participated in this study. Baseline characteristics of the subjects are included in Table 1. Subjects participated in two experimental trials: (1) a placebo and (2) a resveratrol (150 mg/day (99\%); 
resVida ${ }^{\mathrm{TM}}$ ) (provided by DSM Nutritional Products, Ltd) condition, in a randomized double-blind crossover design with a four-week wash-out period. Subjects were instructed to take the first supplement on the day after the baseline measurements (day 1) and the last supplement in the evening on day 29.

To ensure that the subjects adhered to the study protocol and to confirm systemic conversion of resveratrol to dihydroresveratrol (DHR), total (sum of conjugated and unconjugatd resveratrol) and free plasma levels of both compounds were analyzed each week during the 30-day period of resveratrol or placebo supplementation. Whereas no resveratrol or DHR could be detected in the placebo group, both compounds were present in plasma of resveratrol-supplemented subjects (Table 2, levels at day 30 are shown and representative for the whole intervention period). Whereas measurement of plasma resveratrol demonstrated compliance to the study protocol and indicated that the compound was well absorbed, measurement of DHR confirmed that resveratrol was efficiently metabolized, especially as the plasma levels of DHR exceeded those of resveratrol after an overnight fast. No detectable levels of free resveratrol and free DHR were observed in the plasma in both the resveratrol and placebo period, also confirming efficient metabolism. It is important to note that, although the dosage of $150 \mathrm{mg}$ of resveratrol per day is around 133-266× lower compared to the high doses of 200 to $400 \mathrm{mg} / \mathrm{kg} / \mathrm{day}$ used to supplement mice $(14,15)$, plasma resveratrol levels in our human intervention (231.01 $\mathrm{ng} / \mathrm{ml}$ on average during the 30 days) are even higher than those obtained in the mice $(10-120 \mathrm{ng} / \mathrm{ml})(15)$. These differences may be due to different metabolic rates of resveratrol between humans and mice, and therefore suggest that resveratrol may exert its effects at different concentration ranges in different mammals.

To ensure that resveratrol supplementation did not cause adverse effects, we screened plasma and blood of the subjects for several general health parameters. Clinical chemistry, hematology and coagulation analysis revealed no adverse effects after 30 days of resveratrol (see Table $\mathbf{S 1}$ available online). Plasma ALAT concentrations were significantly lower after resveratrol compared to placebo (Table 2). Also, leptin levels and leukocytes in the plasma were significantly lower in resveratrol-treated subjects (Table 2). In line with the reduced leukocyte count, resveratrol also lowered some markers of systemic inflammation, for instance, IL-6 and TNF- $\alpha$ (Table 2). ECGs were inconspicuous and did not raise clinical concern in any subject. No clinical adverse events were reported during the resveratrol supplementation.

Next, we assessed whether resveratrol also exerted positive effects on plasma markers that predict the metabolic risk for development of diabetes and shorter lifespan. After 30 days of resveratrol, plasma glucose and insulin concentrations were lower compared to placebo (Table 2), resulting in a lower HOMA-index, 
indicative of improved insulin sensitivity (Table 2). Plasma triglyceride (TG) concentrations were significantly lower after resveratrol compared to placebo. No differences were observed in plasma non-esterified fatty acids (NEFA) (Table 2).

Table 2. Plasma biochemistry.

\begin{tabular}{|c|c|c|c|}
\hline & Placebo & Resveratrol & P-value \\
\hline Resveratrol (ng/ml) & Not detectable & $182.59 \pm 30.33$ & - \\
\hline Dihydroresveratrol (ng/ml) & Not detectable & $289.14 \pm 93.57$ & - \\
\hline Glucose (mmol/l) & $5.28 \pm 0.15$ & $5.06 \pm 0.13$ & 0.05 \\
\hline Insulin (mU/I) & $11.94 \pm 1.11$ & $10.31 \pm 1.25$ & 0.04 \\
\hline HOMA index & $2.80 \pm 0.20$ & $2.43 \pm 0.24$ & 0.03 \\
\hline Triglycerides (mmol/l) & $2.29 \pm 0.23$ & $2.16 \pm 0.21$ & 0.03 \\
\hline Non-esterified fatty acids ( $\mu \mathrm{mol} / \mathrm{l})$ & $572 \pm 77$ & $621 \pm 38$ & 0.59 \\
\hline Leptin $(\mathrm{ng} / \mathrm{ml})$ & $14.28 \pm 1.98$ & $12.91 \pm 1.84$ & 0.04 \\
\hline Adiponectin $(\mu \mathrm{g} / \mathrm{ml})$ & $6.47 \pm 0.55$ & $6.45 \pm 0.56$ & 0.95 \\
\hline CRP (ng/ml) & $1.52 \pm 0.35$ & $1.33 \pm 0.31$ & 0.11 \\
\hline IL-1 $\beta$ (pg/ml) & $1.33 \pm 0.27$ & $0.94 \pm 0.15$ & 0.20 \\
\hline IL-6 (pg/ml) & $3.13 \pm 0.67$ & $2.42 \pm 0.38$ & 0.09 \\
\hline IL-8 (pg/ml) & $4.94 \pm 0.59$ & $4.28 \pm 0.25$ & 0.19 \\
\hline TNF- $\alpha(p g / m l)$ & $16.15 \pm 2.27$ & $15.14 \pm 2.03$ & 0.04 \\
\hline Leukocytes $\left(10^{9} / I\right)$ & $7.03 \pm 0.44$ & $6.48 \pm 0.39$ & 0.03 \\
\hline ALAT (U/I) & $31.91 \pm 2.21$ & $28.09 \pm 1.54$ & 0.02 \\
\hline
\end{tabular}

Plasma values after 30 days of resveratrol or placebo supplementation. Values are given as means \pm SEM $(n=11)$.

\section{Clinical improvement after resveratrol supplementation}

As calorie restriction was described to associate with reduced absolute 24-hour energy expenditure and sleeping energy expenditure (5), we sought to investigate whether resveratrol treatment induced similar metabolic adaptations. Indeed, resveratrol supplementation for a period of 30 days had a profound effect on resting energy expenditure (Table 3 ). Subjects had a significantly lower sleeping metabolic rate (SMR) compared to placebo, even though 24-hour energy expenditure was similar between resveratrol and placebo (Table 3). Although calorie restriction is known to cause metabolic adaptations in part through weight loss, we found no effect on body mass $(99.9 \pm 3.9 \mathrm{vs}$. $99.6 \pm 2.5 \mathrm{~kg}$, in resveratrol vs. placebo, $p=0.43$ ). Respiratory quotient $(R Q)$ values over 24 hours tended to be higher after resveratrol supplementation, which was largely accounted for by higher $R Q$ values during the daytime (Table 3, the complete time-course for the $R Q$ and the energy expenditure over 24 hours is shown in Table S2). The higher RQ in the resveratrol group, are especially apparent after periods of feeding, and suggest improved metabolic flexibility, i.e. the ability to switch between energy substrates. No differences were observed in diet-induced thermogenesis (DIT) or physical activity index (PAI). 
Next, we investigated whether resveratrol also exerted favorable effects on other general health parameters. As long-term calorie restriction in humans $(8,41)$ and resveratrol treatment in mice (15) was shown to ameliorate cardiac function, we examined whether resveratrol also exerts beneficial effects on blood pressure. Resveratrol significantly lowered systolic blood pressure by $\sim 5 \mathrm{mmHg}$, while resveratrol had no effect on diastolic blood pressure (Table 3). Mean arterial pressure was significantly lower after resveratrol supplementation (Table 3).

Table 3. Clinical improvement after resveratrol.

\begin{tabular}{lccc}
\hline & Placebo & Resveratrol & P-value \\
\hline 24h Respiratory Quotient & $0.89 \pm 0.007$ & $0.91 \pm 0.006$ & 0.09 \\
Respiratory Quotient daytime & $0.89 \pm 0.004$ & $0.91 \pm 0.003$ & 0.001 \\
Respiratory Quotient nighttime & $0.87 \pm 0.007$ & $0.88 \pm 0.009$ & 0.18 \\
24h Energy Expenditure (MJ/d) & $11.86 \pm 0.29$ & $11.91 \pm 0.29$ & 0.64 \\
Sleeping Metabolic Rate first night (MJ/d) & $8.09 \pm 0.24$ & $7.75 \pm 0.23$ & 0.007 \\
Sleeping Metabolic Rate second night (MJ/d) & $8.06 \pm 0.22$ & $7.90 \pm 0.18$ & 0.06 \\
Diet-induced Thermogenesis (MJ/d) & $1.02 \pm 0.13$ & $1.14 \pm 0.17$ & 0.33 \\
Physical Activity Index & $1.49 \pm 0.02$ & $1.50 \pm 0.01$ & 0.37 \\
Systolic blood pressure (mmHg) & $130.5 \pm 2.7$ & $124.7 \pm 3.1$ & 0.006 \\
Diastolic blood pressure (mmHg) & $81.6 \pm 2.8$ & $80.0 \pm 2.9$ & 0.18 \\
Mean arterial pressure (mmHg) & $97.9 \pm 2.7$ & $94.9 \pm 2.9$ & 0.02 \\
\hline
\end{tabular}

Energy metabolism $(n=10)$, and blood pressure $(n=11)$ after 30 days of resveratrol or placebo supplementation. Values are given as means \pm SEM.

\section{Postprandial substrate utilization and tissue lipolysis}

Mounting evidence implicates SIRT1 as a switch in substrate utilization, for instance from glucose to fatty acid oxidation under low-nutrient conditions $(9,24)$. Therefore, we next investigated whether resveratrol is capable to increase fat oxidation in adipose tissue and skeletal muscle after a mixed meal, by stimulating tissue lipolysis. The peak glucose and insulin responses after consuming a liquid test meal were reached after $30 \mathrm{~min}$ for placebo and after $60 \mathrm{~min}$ for resveratrol (Figure 1A,B). There was a tendency for NEFA (AUC 4h-6h, $p=0.097$ ) and free glycerol (AUC 4h-6h, $p=0.06$ ) to be lower in the late postprandial phase after resveratrol (Figure 1C,D). Resveratrol did not alter postprandial TG and lactate responses (data not shown). Using the microdialysis technique, ethanol out/in ratios were determined to measure blood flow. At baseline, ethanol out/in ratios were not different after resveratrol supplementation compared to placebo, both in adipose tissue $(0.81 \pm 0.02$ vs. $0.81 \pm 0.02$, respectively) and skeletal muscle $(0.46$ \pm 0.02 vs. $0.49 \pm 0.02$, respectively). Furthermore, resveratrol did not affect postprandial adipose tissue and skeletal muscle blood flow (data not shown). The interstitial glycerol concentration in adipose tissue was slightly lower in the resveratrol condition in the mid- and late postprandial phase, although it did not 
reach statistical significance (AUC $2 h-4 h, p=0.14 ; A \cup C$ 4h-6h, $p=0.13$ ) (Figure 1E). There were no differences in interstitial glucose, pyruvate and lactate responses in adipose tissue between placebo and resveratrol (data not shown). For skeletal muscle, there were no differences in interstitial glucose, pyruvate, lactate and glycerol concentrations (glycerol data shown Figure 1F). Interestingly, energy expenditure in the postprandial period was significantly lower after resveratrol supplementation (AUC, $\mathrm{p}=0.02$ ) (Figure 1G). During the late postprandial phase (4h-6h), RQ tended to be higher ( $A \cup C$ 4h-6h, $p=0.07$ ) (Figure 1H) and fat oxidation was decreased in the resveratrol condition (AUC 4h-6h, $p=0.007$ ) (Figure 1I), again suggesting improved metabolic flexibility.
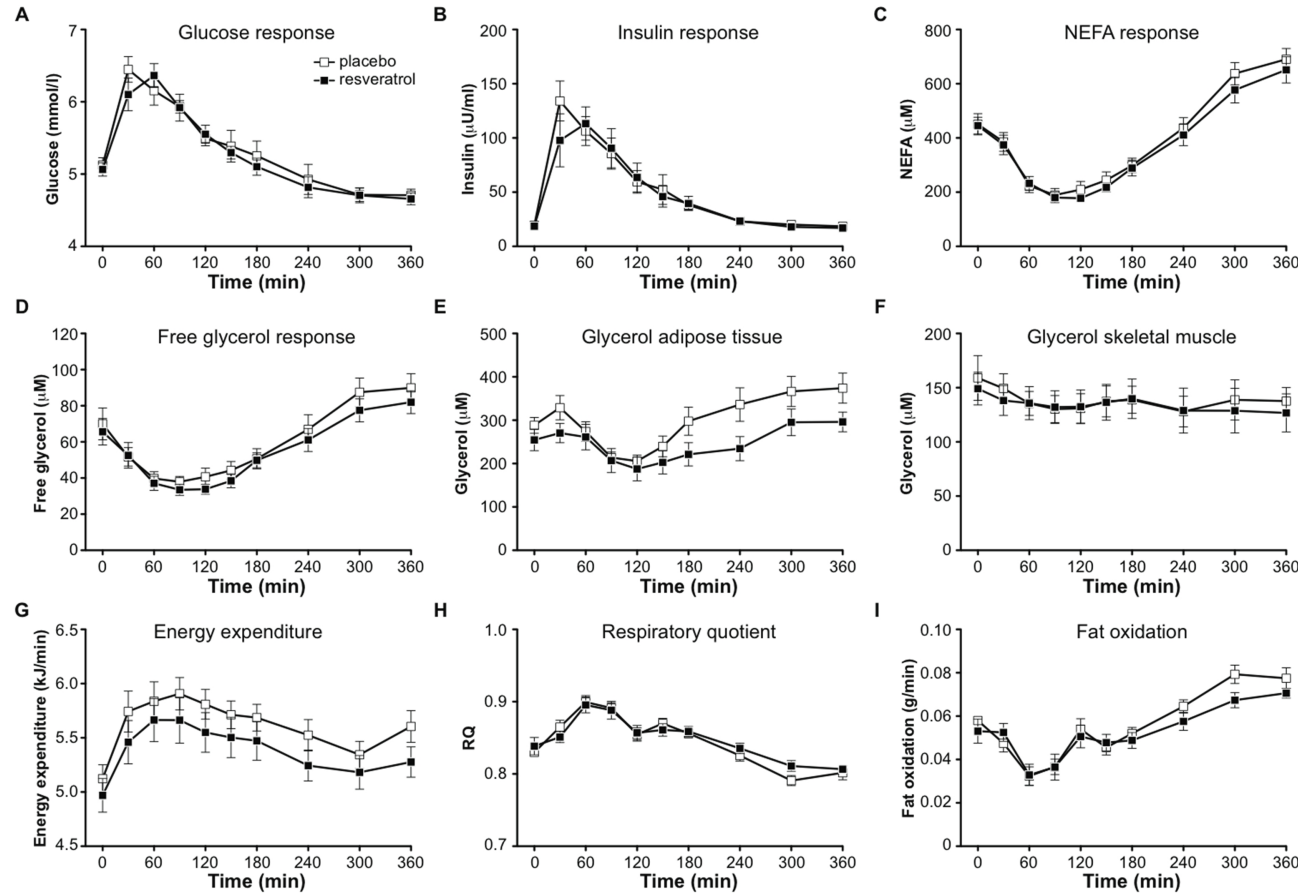

Figure 1. Resveratrol decreases postprandial energy expenditure and lowers adipose tissue lipolysis. Data are obtained on whole body (A-D), by microdialysis (E-F) and indirect calorimetry (G-I). Plasma metabolites during the postprandial microdialysis test after 30 days of placebo or resveratrol supplementation: (A) glucose response (B) insulin response (C) NEFA response (D) free glycerol response. Interstitial glycerol responses in (E) adipose tissue and $(F)$ skeletal muscle during the postprandial microdialysis test. Indirect calorimetry results during the postprandial microdialysis test: $(G)$ energy expenditure $(\mathrm{H})$ respiratory quotient $(\mathrm{I})$ fat oxidation. Open squares, placebo; Black squares, resveratrol. Values are given as means $\pm \operatorname{SEM}(n=10)$.

\section{Molecular mechanism of resveratrol treatment}

To clarify the mechanism of action of resveratrol, we performed microarray analysis on vastus lateralis muscle biopsies. We found 469 genes differentially expressed between placebo and RSV-supplementation, 219 of which were increased and 250 
decreased (Figure 2A). We performed gene set enrichment analysis (GSEA) to define the pathways that were affected by resveratrol, and to compare the expression profile with previous studies in mouse (15). Several gene sets related to mitochondrial oxidative phosphorylation were upregulated, whereas pathways linked to inflammation were downregulated upon resveratrol supplementation. Indeed, when analyzing expression of the individual genes in the oxidative phosphorylation-related gene sets, we observed a marked increase (Figure 2B). Importantly, these effects are highly similar to the effects observed in mice treated with resveratrol (Figure $\mathbf{2 C}$ ), even though the effects in the latter are more pronounced, probably due to longer treatment time and their identical genetic and environmental background.

Since resveratrol was shown to activate AMPK, we measured levels of phosphorylated AMPK on the $\operatorname{Thr}^{172}$ residue of the $\alpha$-subunit-a marker for its activation. Indeed, increased AMPK phosphorylation was observed in muscle biopsies of the resveratrol-treated subjects (Figure 3A, S1A).

A

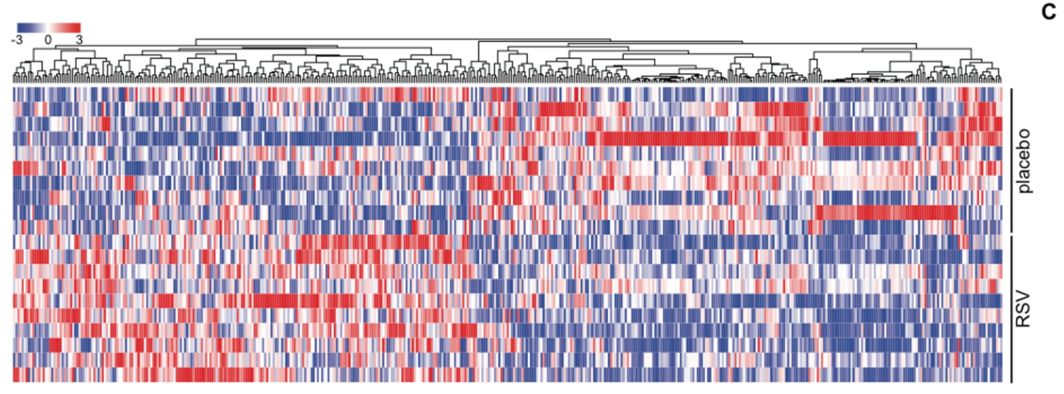

B
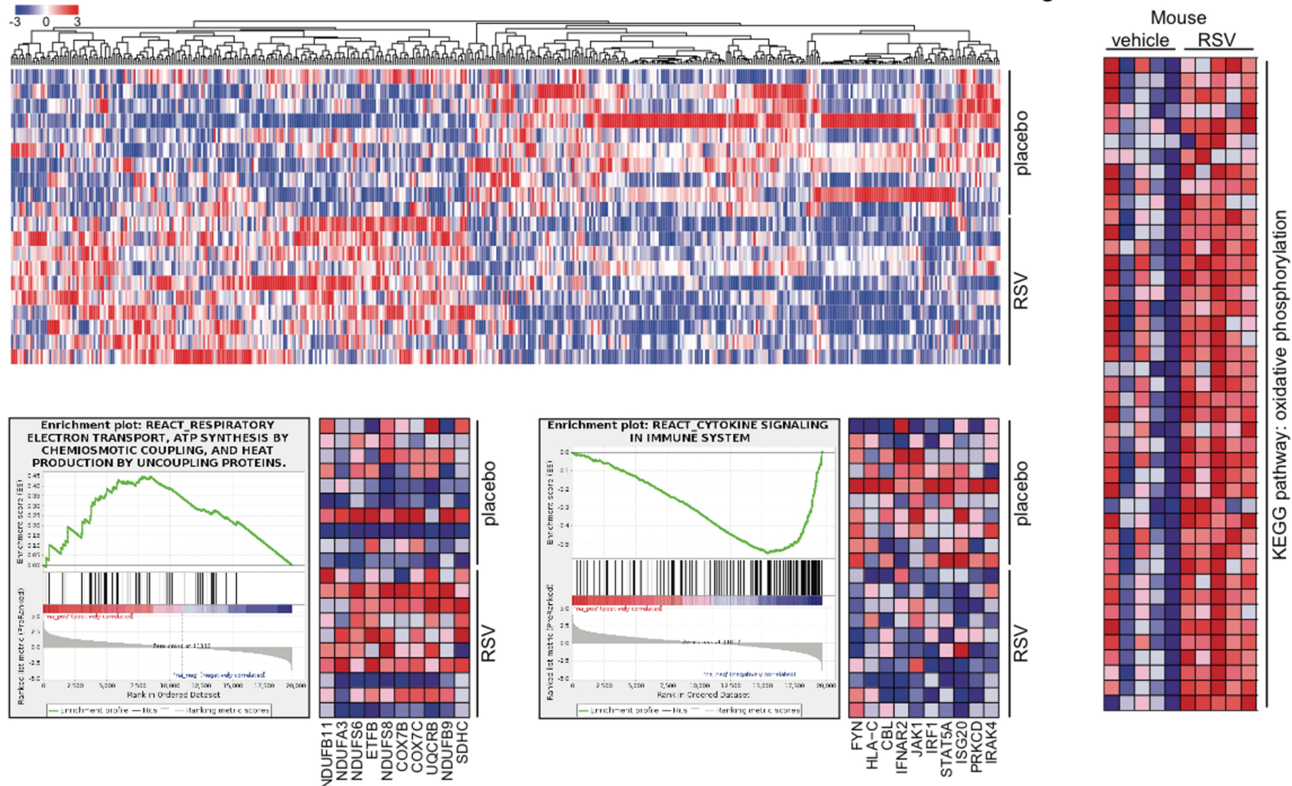

Figure 2. Resveratrol increases oxidative phosphorylation gene expression in man and mice. (A) One-way hierarchical clustering of genes significantly changed in a microarray of vastus lateralis of subjects receiving resveratrol (RSV) or placebo. In muscle, 469 genes were differentially expressed between placebo and RSV ( $n=10)$. (B) Gene set enrichment analysis (GSEA) of muscle microarray revealed that gene sets related to mitochondrial oxidative phosphorylation were significantly enriched following resveratrol supplementation in humans. (C) Previously published microarray data of mice treated with 400 mpkd RSV (15) were reanalyzed by GSEA, showing a marked enrichment of oxidative phosphorylation genes. 


\section{Mitochondrial metabolism}

Our microarray data clearly suggest strong involvement of mitochondrial function in the beneficial effects of resveratrol. We hence performed some more detailed analyses of mitochondrial function. Mitochondrial DNA copy number in the resveratrol condition was similar to the values obtained after placebo (994.8 \pm 101.4 vs. $1074.7 \pm 109.9 \mathrm{AU}$ in resveratrol vs. placebo, $p=0.20$ ). Also, neither the protein content of the individual structural components of the individual OXPHOS complexes nor the mean of all these complexes revealed differences in mitochondrial density in resveratrol compared to placebo supplementation (Figure 3B, S1B). On the other hand, protein content of the AMPK downstream effector SIRT1 (22), as well as the mitochondrial master regulator PGC1 $\alpha$ was significantly increased after 30 days of resveratrol (Figure 3C, S1C), as was citrate synthase activity (Figure 3D)—a common marker for mitochondrial activity.

Mitochondrial function can also be assessed in vivo by measurement of phosphocreatine (PCr) recovery rate after exercise (30). To this end, single leg extension exercise was performed for 5 min with a weight corresponding to $60 \%$ of maximal capacity, which was determined with an incremental maximal test on the same ergometer on a different day. Low- to medium- intensity exercise was chosen to prevent acidification of the muscle, which is known to affect $\mathrm{PCr}$ recovery. Spectra were acquired 2 minutes before, during the 5 minutes of knee extension exercise and during the subsequent 5 minutes of recovery (rest). As targeted, $\mathrm{PCr}$ levels decreased during the knee-extension exercise until a steady state was reached. However, mean $\mathrm{PCr}$ recovery half time (PCr- $\left.\mathrm{t}_{1 / 2}\right)$ was unchanged by resveratrol compared to placebo (Figure 3E).

To functionally characterize mitochondrial oxidative phosphorylation in more detail, we measured mitochondrial respiration using different substrate combinations. State 2 respiration (i.e., respiration in the presence of exogenous substrates alone) was not different between resveratrol versus placebo on any of the substrate combinations studied (data not shown). ADP-stimulated (state 3) respiration on a lipid substrate (malate + octanoyl-carnitine, $\mathrm{MO}$ ) tended to be higher after resveratrol supplementation $(p=0.075$, Figure $3 F)$. State 3 respiration upon the Complex I-linked substrates malate + glutamate (MG) was unaffected (Figure 3G). Respiration upon parallel electron input to both Complex I and II was $\sim 10 \%$ higher upon resveratrol. Thus, state 3 respiration upon malate + octanoyl-carnitine + glutamate (MOG) was significantly higher in subjects receiving resveratrol (Figure 3H). Similar differences were observed for state 3 respiration upon malate + octanoyl-carnitine + glutamate + succinate (MOGS) (Figure $\mathbf{3 H}$ ), but not in the absence of octanoyl-carnitine (malate + glutamate + succinate, MGS) (Figure $\mathbf{3 H}$ ). Maximal FCCP-induced uncoupled respiration, measured in the presence of octanoyl-carnitine, reflecting the maximal capacity of the electron transport chain, 
was also higher after 30 days of resveratrol (Figure 3I). State 40 respiration (reflecting mitochondrial proton leak) was similar between resveratrol and placebo condition (Figure $\mathbf{3} \mathbf{J}$ ). Similar results were obtained if respiration rates were not corrected for mtDNA copy number.

A

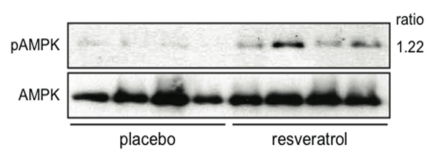

B

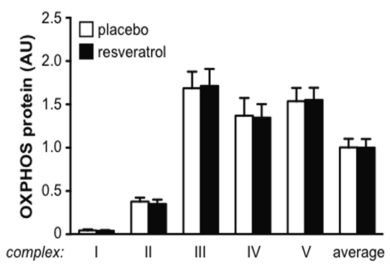

C

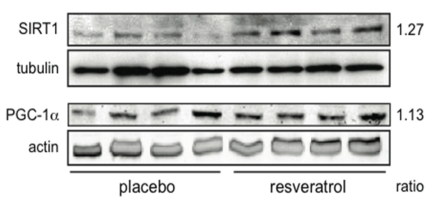

D

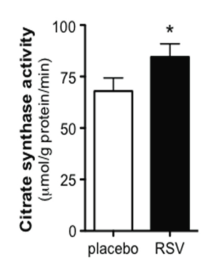

E

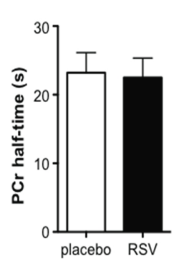

$\mathbf{F}$

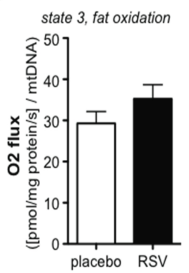

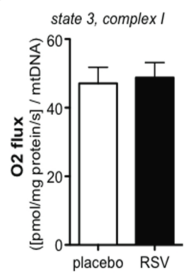

H

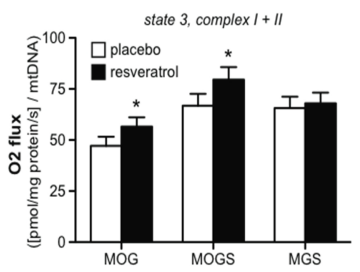

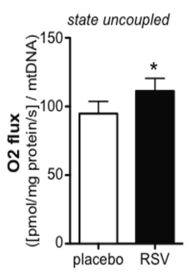

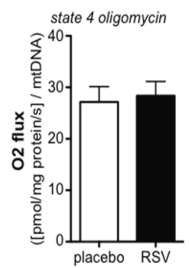

Figure 3. Resveratrol increases AMPK activity, increases mitochondrial efficiency and respiration on fatty acid substrates. After 30 days of resveratrol or placebo, a muscle biopsy was obtained from the vastus lateralis muscle. Of total protein extracts from the vastus lateralis muscle, 50 $\mu \mathrm{g}$ of protein was used to check the expression levels of several proteins by western blotting. (A) The level of phosphorylation of residue Thr ${ }^{172}$ of the AMPK $\alpha$ subunit (top panel), indicative of AMPK activity, by Western blotting in a total of 9 subjects per group (representative subset is shown). Total level of the AMPK $\alpha$ protein are shown in the bottom panel. The ratio resveratrol / placebo is shown on the right of panel A. Relative quantification of the ratio of pAMPK/ AMPK is shown in S1A. (B) The protein content of the individual complexes of the electron transport chain are quantified by Western blotting in vastus lateralis muscle of 11 subjects per group. An antibody cocktail that detects all five complexes is used, and the data are represented as mean \pm SEM for the individual complexes as well as for the average expression of all the complexes. A representative Western blot is included in S1B.

(C) SIRT1 and PGC1 $\alpha$ protein levels in vastus lateralis muscle by Western blotting ( $n=8$ for SIRT1 and $\mathrm{n}=11$ for $\mathrm{PGC1} \alpha$, a representative subset is shown). The relative expression of both proteins is corrected for the loading control, tubulin for SIRT1 and actin for PGC1 $\alpha$. The ratio of resveratrol / placebo is shown of the right of panel $C$. The relative quantification of the western blots is shown in S1C. (D) Citrate Synthase activity was significantly increased in vastus lateralis muscle of resveratrol (RSV)-treated subjects $(n=11),{ }^{*} p<0.05$. (E) In vivo mitochondrial function expressed as $\mathrm{PCr}$ half-time $(n=11)$. Evaluation of ex vivo mitochondrial function in vastus lateralis muscle: $(F)$ ADP-stimulated respiration (state 3) upon a lipid substrate. (G) State 3 respiration fueled by Complex I-linked substrates. (H) State 3 respiration upon parallel electron input into Complex I and II, ${ }^{*} p<0.05$. (I) Maximally uncoupled respiration upon FCCP, * $p<0.05$. (J) Mitochondrial respiration uncoupled from ATP synthesis (state 40 ). White bars, placebo; black bars, resveratrol. Values are given as means \pm SEM $(n=10)$. M, malate; O, octanoyl-carnitine; G, glutamate; S, succinate. 


\section{Lipid accumulation in liver and skeletal muscle}

Since our data show that resveratrol clearly induced a shift in mitochondrial substrate usage, we tested whether this also reflected in liver and skeletal muscle lipid accumulation. Intrahepatic lipid (IHL) content was lower after 30 days of resveratrol supplementation in comparison to placebo (Figure 4A). This was paralleled by lower plasma ALAT values, as mentioned before, both indicating improved liver function. The mean intramyocellular lipid (IMCL) area fraction was $\sim 2$-fold higher in the resveratrol group (Figure 4B). The increase in lipid accumulation was more pronounced in fibers identified as slow, oxidative (type 1) fibers than in type 2 muscle fibers (Figure 4B).

A
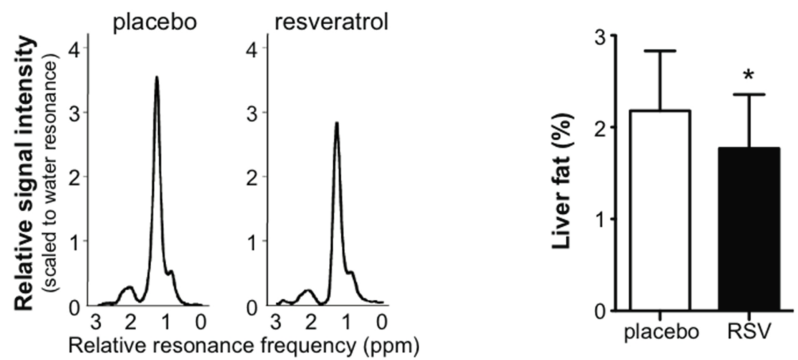

B
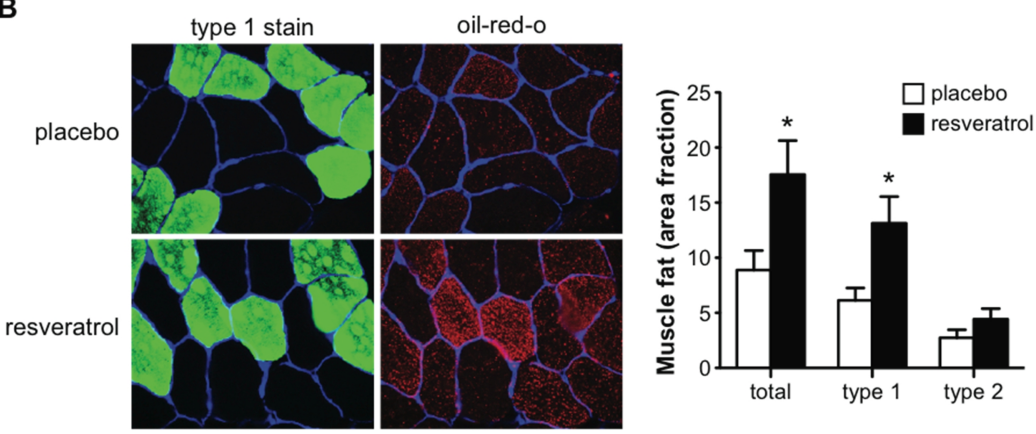

Figure 4. Resveratrol decreases intrahepatic lipid content but increases intramyocellular lipid content. Ectopic lipid deposition after 30 days of placebo or resveratrol supplementation. (A) Typical lipid regions of hepatic ${ }^{1} \mathrm{H}-\mathrm{MR}$ spectra from a subject receiving placebo (left) and resveratrol (right) at $3 \mathrm{~T}$, scaled to the water resonance (reference). The $\mathrm{CH}_{2}$ peak is used for quantification, the area under the curve is proportional to the lipid content in the liver. Quantification revealed that intrahepatic lipid content was reduced by resveratrol $(n=9),{ }^{*} p<0.05$. (B) Images of representative cross section of the vastus lateralis muscle from a subject receiving placebo (top) and resveratrol (bottom). Sections are stained for intramyocellular lipids with Oil Red O staining (in red), muscle laminin (in blue) and type 1 muscle fibers (in green) (400x magnification). IMCL content significantly increased by resveratrol treatment in muscle fibers, which was mostly accounted for by an increased lipid storage in type I muscle fibers $(n=10),{ }^{\star} p<0.05$. White bars, placebo; black bars, resveratrol. Values are given as means \pm SEM. 


\section{Discussion}

Resveratrol, which was discovered in a small-molecule screen as a potent SIRT1 activator (19), has been extensively studied in animal and cellular studies with promising results $(14,15)$. Here, we show that resveratrol supplementation in humans exerted favorable metabolic adaptations that in many aspects mimic the effects of calorie restriction and/or endurance training $(5,6,8,42,43)$. These metabolic adaptations include a reduction in sleeping metabolic rate, blood pressure and hepatic lipid content, an improvement in skeletal muscle intrinsic mitochondrial function and several plasma markers of general health, an increase in intramyocellular lipid content, as well as an increase in skeletal muscle PGC1 $\alpha$ protein content. These data extend findings, which so far have only been observed in cell and rodent models, to the human situation, showing that resveratrol has promising beneficial metabolic effects, and suggests that resveratrol has the potential to improve metabolic health in subjects at risk for developing the metabolic syndrome.

Resveratrol exerts significant effects on energy metabolism. Sleeping metabolic rate (SMR) was significantly lower following 30 days of resveratrol supplementation, without changing 24-hour energy expenditure. It should be noted that SMR is the component of human energy metabolism that is most sensitive to metabolic changes-as it is not affected by physical activity—and small differences in sleeping energy expenditure can be detected with high accuracy. In line, we observed that basal and postprandial energy expenditure was also lower after 30 days of resveratrol supplementation. The $2-4 \%$ reduction in energy expenditure upon resveratrol treatment is consistent with the effects observed after calorie restriction (6\% reduction) $(5,44)$.

It is important to note that basal and postprandial energy expenditure was reduced by resveratrol in our human study rather than increased, as was true for mice (15). Although our energy expenditure data are opposite to the effects seen in mice, a lowering of resting and sleeping metabolic rate is likely a reflection of improved metabolic efficiency, and completely in line with the endurance training or calorie restriction-like effects of resveratrol $(5,44)$. Although the dose used in our human study was $\sim 200$ fold lower than doses used in the mouse studies, we reached similar plasma resveratrol concentrations. We cannot exclude, however, that metabolism of resveratrol is different between mouse and man, and, possibly more importantly, that timing of treatment (30 days in our study vs 4-6 months in mice $(14,15))$ significantly impacts physiological outcome.

Prolonged calorie restriction, during six months, has also been suggested to increase the expression of genes encoding proteins involved in mitochondrial biogenesis and function (43). Similarly, our microarray data indicate increased 
mitochondrial gene expression in muscle following resveratrol supplementation. In fact, we showed that this induction is likely to be mediated by AMPK—which is activated by resveratrol-and resulted in increased SIRT1 and PGC1 $\alpha$ protein content, accompanied by increased citrate synthase activity, suggesting that mitochondrial activity was effectively improved. Moreover, detailed mitochondrial characterization revealed that resveratrol had beneficial effects on mitochondrial respiration when octanoyl-carnitine was used a substrate, but not when only glutamate was used. These data suggest that resveratrol specifically improves muscle fat oxidative mitochondrial capacity. Interestingly, we have recently found comparable effects in PGC1 $\alpha$ overexpressing mice, showing an improved intrinsic mitochondrial function (i.e. respiration per mitochondrion) but only when fatty acids are used as a substrate (45). The fact that mtDNA copy number, OXPHOS protein content, and $\mathrm{PCr}$ recovery were not changed in resveratrol-treated subjects suggests that 30 days resveratrol treatment mostly affects mitochondrial efficiency, not abundance. It is well possible that more long-term treatment would cause these parameters to change as well.

Interestingly, IMCL content was markedly increased after 30 days of resveratrol supplementation. Together with the improvement in muscle fat oxidative capacity, and the other beneficial metabolic adaptations like lowering of circulating TG and glucose levels, the present data hint towards endurance training-like effects of resveratrol $(46,47)$. Consistent with data in rats, where resveratrol has been shown to reduce hepatic lipid synthesis $(48,49)$, we observed reduced IHL content. Recent data suggest that endurance training is also able to lower IHL content (50), as was calorie restriction, although this effect was mainly attributed to a reduction in body weight $(6,7)$. Taken together with the reduced plasma TG and increased muscle fatty acid oxidation, we hypothesize that fat is liberated from peripheral depots, to be metabolized by the muscle. Again, here our data suggest that resveratrol mimics the effect of calorie restriction and endurance training. Also, we have investigated postprandial metabolism, but only found a reduction in total energy expenditure that was reflected by a lower fat oxidation during the late postprandial phase-a change that is reminiscent of the role of SIRT1 in regulating the efficient switch in energy substrate utilization $(22,23)$. Although postprandial lipolysis tended down, this does not refute our hypothesis of increased fat mobilization, but rather confirms the idea of improved metabolic flexibility.

A striking finding in our study was the resveratrol-induced reduction of systolic blood pressure by $5 \mathrm{mmHg}$. In rodents, resveratrol is also vasoactive and has been shown to cause vasodilatation and to improve aortic endothelial function in diabetic mice (51), to reduce heart rate (15) and even to lower blood pressure (52). Also in human obese subjects endothelial function and cardiovascular health, dosedependently improved after one week of resveratrol supplementation (53). 
Similarly, healthy non-obese individuals also improved their cardiovascular risk after six months of calorie restriction as evidenced by a reduction in blood pressure (8). Another indication that global health was improved upon resveratrol treatment was the decreased expression levels of genes of inflammatory pathways, as were plasma levels of several inflammatory markers, and leukocyte numbers.

Our data also point towards favorable metabolic adaptations after 30 days of resveratrol supplementation in obese subjects. Indeed, HOMA-index was improved after resveratrol, suggesting favorable effects on insulin sensitivity. These beneficial effects of resveratrol on metabolism are in concordance with several findings of resveratrol supplementation in animals (14-16, 22, 28).

In conclusion, we demonstrate beneficial effects of resveratrol supplementation for 30 days on the metabolic profile in healthy obese males, which seems to reflect effects observed during calorie restriction (Table S3). Although most of the effects that we observed were modest, they were very consistently pointing towards beneficial metabolic adaptations. Furthermore, there were no effects on safety parameters and no adverse events were reported. Therefore, resVida was safe and well tolerated at the tested concentration. Future studies should investigate the long-term and dose-dependent metabolic effects of resveratrol supplementation in order to further establish whether resveratrol supplementation has the potential to overcome the metabolic aberrations that are associated with obesity in humans. 


\section{Acknowledgements}

This study was funded by Top Institute Food and Nutrition. TI Food and Nutrition, formerly known as WCFS, is a unique public/private partnership that generates vision on scientific breakthroughs in food and nutrition, resulting in the development of innovative products and technologies that respond to consumer demands for safe, tasty and healthy foods. Partners are major Dutch food companies and research organizations. A VICl (grant 918.96.618) and a VENI (grant 916.11.136) for innovative research from the Netherlands Organization for Scientific Research (NWO) supports the work of P. Schrauwen and V.SchrauwenHinderling, respectively. The work in the Auwerx laboratory is supported by the European Research Council (Sirtuins; ERC-2008-AdG231-118), Swiss National Science Foundation, the Velux foundation, and Ecole Polytechnique Fédérale. JA is the Nestle Chair in Energy Metabolism. RHH is supported by a Rubicon fellowship of the Netherlands Organization for Scientific Research.

The authors would like to thank DSM Nutritional Products Ltd., Kaiseraugst, Switzerland for providing us with the resVida ${ }^{\mathrm{TM}}$ and placebo capsules and for performing the resveratrol and dihydroresveratrol analysis. The authors thank Jos Stegen for his excellent technical assistance with the biochemical analysis. 


\section{References}

1. Crews DE. Artificial environments and an aging population: designing for age-related functional losses. J Physiol Anthropol Appl Human Sci 2005;24(1):103-9.

2. Barger $\mathrm{JL}$, Walford RL, Weindruch R. The retardation of aging by caloric restriction: its significance in the transgenic era. Exp Gerontol 2003;38(11-12):1343-51.

3. Goodrick CL, Ingram DK, Reynolds MA, Freeman JR, Cider N. Effects of intermittent feeding upon body weight and lifespan in inbred mice: interaction of genotype and age. Mech Ageing Dev 1990;55(1):69-87.

4. McCay CM, Crowell MF, Maynard LA. The effect of retarded growth upon the length of life span and upon the ultimate body size The Journal of nutrition 1935;10:63-79.

5. Heilbronn LK, de Jonge L, Frisard MI, et al. Effect of 6-month calorie restriction on biomarkers of longevity, metabolic adaptation, and oxidative stress in overweight individuals: a randomized controlled trial. JAMA 2006;295(13):1539-48.

6. Larson-Meyer DE, Heilbronn LK, Redman LM, et al. Effect of calorie restriction with or without exercise on insulin sensitivity, beta-cell function, fat cell size, and ectopic lipid in overweight subjects. Diabetes Care 2006;29(6):1337-44.

7. Lim EL, Hollingsworth KG, Aribisala BS, Chen MJ, Mathers JC, Taylor R. Reversal of type 2 diabetes: normalisation of beta cell function in association with decreased pancreas and liver triacylglycerol. Diabetologia 2011.

8. Lefevre M, Redman LM, Heilbronn LK, et al. Caloric restriction alone and with exercise improves CVD risk in healthy non-obese individuals. Atherosclerosis 2009;203(1):206-13.

9. Canto C, Auwerx J. Caloric restriction, SIRT1 and longevity. Trends Endocrinol Metab 2009;20(7):325-31.

10. Lin SJ, Defossez PA, Guarente L. Requirement of NAD and SIR2 for life-span extension by calorie restriction in Saccharomyces cerevisiae. Science 2000;289(5487):2126-8.

11. Tissenbaum HA, Guarente L. Increased dosage of a sir-2 gene extends lifespan in Caenorhabditis elegans. Nature 2001;410(6825):227-30.

12. Rogina B, Helfand SL. Sir2 mediates longevity in the fly through a pathway related to calorie restriction. Proc Natl Acad Sci U S A 2004;101(45):15998-6003.

13. Burnett $C$, Valentini S, Cabreiro F, et al. Absence of effects of Sir2 over-expression on lifespan in C. elegans and drosophila. Nature 2011;477:482-5.

14. Baur JA, Pearson KJ, Price NL, et al. Resveratrol improves health and survival of mice on a high-calorie diet. Nature 2006;444(7117):337-42.

15. Lagouge M, Argmann C, Gerhart-Hines Z, et al. Resveratrol improves mitochondrial function and protects against metabolic disease by activating SIRT1 and PGC-1alpha. Cell 2006;127(6):1109-22.

16. Pearson KJ, Baur JA, Lewis KN, et al. Resveratrol delays age-related deterioration and mimics transcriptional aspects of dietary restriction without extending life span. Cell Metab 2008;8(2):157-68.

17. Herranz D, Munoz-Martin M, Canamero M, et al. Sirt1 improves healthy ageing and protects from metabolic syndrome-associated cancer. Nat Commun 2010;1:3.

18. Rutanen J, Yaluri N, Modi S, et al. SIRT1 mRNA expression may be associated with energy expenditure and insulin sensitivity. Diabetes 2010;59(4):829-35.

19. Howitz KT, Bitterman KJ, Cohen HY, et al. Small molecule activators of sirtuins extend Saccharomyces cerevisiae lifespan. Nature 2003;425(6954):191-6.

20. Pacholec M, Bleasdale JE, Chrunyk B, et al. SRT1720, SRT2183, SRT1460, and resveratrol are not direct activators of SIRT1. J Biol Chem 2010;285(11):8340-51.

21. Beher D, Wu J, Cumine S, et al. Resveratrol is not a direct activator of SIRT1 enzyme activity. Chem Biol Drug Des 2009;74(6):619-24.

22. Canto $\mathrm{C}$, Gerhart-Hines Z, Feige JN, et al. AMPK regulates energy expenditure by modulating NAD+ metabolism and SIRT1 activity. Nature 2009;458(7241):1056-60.

23. Canto C, Jiang LQ, Deshmukh AS, et al. Interdependence of AMPK and SIRT1 for metabolic adaptation to fasting and exercise in skeletal muscle. Cell Metab 2010;11(3):213-9.

24. Feige JN, Lagouge M, Canto C, et al. Specific SIRT1 activation mimics low energy levels and protects against diet-induced metabolic disorders by enhancing fat oxidation. Cell Metab 2008;8(5):347-58.

25. Hawley SA, Ross FA, Chevtzoff $C$, et al. Use of cells expressing gamma subunit variants to identify diverse mechanisms of AMPK activation. Cell Metab 2010;11(6):554-65. 
26. Um JH, Park SJ, Kang $\mathrm{H}$, et al. AMP-activated protein kinase-deficient mice are resistant to the metabolic effects of resveratrol. Diabetes 2010;59(3):554-63.

27. Dai H, Kustigian L, Carney D, et al. SIRT1 activation by small molecules: kinetic and biophysical evidence for direct interaction of enzyme and activator. J Biol Chem 2010;285(43):32695-703.

28. Sun $\mathrm{C}$, Zhang $\mathrm{F}, \mathrm{Ge} X$, et al. SIRT1 improves insulin sensitivity under insulin-resistant conditions by repressing PTP1B. Cell Metab 2007;6(4):307-19.

29. Kuipers H, Verstappen FT, Keizer HA, Geurten P, van Kranenburg G. Variability of aerobic performance in the laboratory and its physiologic correlates. Int J Sports Med 1985;6(4):197201.

30. Schrauwen-Hinderling VB, Kooi ME, Hesselink MK, et al. Impaired in vivo mitochondrial function but similar intramyocellular lipid content in patients with type 2 diabetes mellitus and BMI-matched control subjects. Diabetologia 2007;50(1):113-20.

31. Schoffelen PF, Westerterp KR, Saris WH, Ten Hoor F. A dual-respiration chamber system with automated calibration. J Appl Physiol 1997;83(6):2064-72.

32. Schrauwen P, van Marken Lichtenbelt WD, Saris WH, Westerterp KR. Changes in fat oxidation in response to a high-fat diet. Am J Clin Nutr 1997;66(2):276-82.

33. Hamilton G, Yokoo T, Bydder M, et al. In vivo characterization of the liver fat (1)H MR spectrum. NMR Biomed 2011:in press.

34. Vanhamme L, van den Boogaart A, Van Huffel S. Improved method for accurate and efficient quantification of MRS data with use of prior knowledge. J Magn Reson 1997;129(1):35-43.

35. Naressi A, Couturier C, Devos JM, et al. Java-based graphical user interface for the MRUI quantitation package. MAGMA 2001;12(2-3):141-52.

36. Phielix E, Schrauwen-Hinderling VB, Mensink M, et al. Lower intrinsic ADP-stimulated mitochondrial respiration underlies in vivo mitochondrial dysfunction in muscle of male type 2 diabetic patients. Diabetes 2008;57(11):2943-9.

37. Boushel R, Gnaiger E, Schjerling P, Skovbro M, Kraunsoe R, Dela F. Patients with type 2 diabetes have normal mitochondrial function in skeletal muscle. Diabetologia 2007;50(4):7906.

38. Hoeks J, van Herpen NA, Mensink M, et al. Prolonged fasting identifies skeletal muscle mitochondrial dysfunction as consequence rather than cause of human insulin resistance. Diabetes 2010;59(9):2117-25.

39. Koopman R, Schaart G, Hesselink MK. Optimisation of oil red O staining permits combination with immunofluorescence and automated quantification of lipids. Histochem Cell Biol 2001;116(1):63-8.

40. Goossens GH, Blaak EE, Saris WH, van Baak MA. Angiotensin II-induced effects on adipose and skeletal muscle tissue blood flow and lipolysis in normal-weight and obese subjects. $\mathrm{J}$ Clin Endocrinol Metab 2004;89(6):2690-6.

41. Meyer TE, Kovacs SJ, Ehsani AA, Klein S, Holloszy JO, Fontana L. Long-term caloric restriction ameliorates the decline in diastolic function in humans. J Am Coll Cardiol 2006;47(2):398-402.

42. Larson-Meyer DE, Newcomer BR, Heilbronn LK, et al. Effect of 6-month calorie restriction and exercise on serum and liver lipids and markers of liver function. Obesity (Silver Spring) 2008;16(6):1355-62.

43. Civitarese AE, Carling S, Heilbronn LK, et al. Calorie restriction increases muscle mitochondrial biogenesis in healthy humans. PLoS Med 2007;4(3):e76.

44. Martin CK, Heilbronn LK, de Jonge L, et al. Effect of calorie restriction on resting metabolic rate and spontaneous physical activity. Obesity (Silver Spring) 2007;15(12):2964-73.

45. Hoeks J, Arany Z, Phielix E, Moonen-Kornips E, Hesselink MK, Schrauwen P. Enhanced lipid -but not carbohydrate- supported mitochondrial respiration in skeletal muscle of PGC-1alpha overexpressing mice. J Cell Physiol 2011:in press.

46. Dube JJ, Amati F, Stefanovic-Racic M, Toledo FG, Sauers SE, Goodpaster BH. Exerciseinduced alterations in intramyocellular lipids and insulin resistance: the athlete's paradox revisited. Am J Physiol Endocrinol Metab 2008;294(5):E882-8.

47. Meex RC, Schrauwen-Hinderling VB, Moonen-Kornips E, et al. Restoration of muscle mitochondrial function and metabolic flexibility in type 2 diabetes by exercise training is paralleled by increased myocellular fat storage and improved insulin sensitivity. Diabetes 2010;59(3):572-9.

48. Arichi H, Kimura Y, Okuda H, Baba K, Kozawa M, Arichi S. Effects of stilbene components of the roots of Polygonum cuspidatum Sieb. et Zucc. on lipid metabolism. Chem Pharm Bull (Tokyo) 1982;30(5):1766-70. 
49. Ahn J, Cho I, Kim S, Kwon D, Ha T. Dietary resveratrol alters lipid metabolism-related gene expression of mice on an atherogenic diet. J Hepatol 2008;49(6):1019-28.

50. Kantartzis K, Thamer C, Peter A, et al. High cardiorespiratory fitness is an independent predictor of the reduction in liver fat during a lifestyle intervention in non-alcoholic fatty liver disease. Gut 2009;58(9):1281-8.

51. Wang N, Ko SH, Chai W, et al. Resveratrol recruits rat muscle microvasculature via a nitric oxide-dependent mechanism that is blocked by TNFalpha. Am J Physiol Endocrinol Metab 2011;300(1):E195-201.

52. Rivera L, Moron R, Zarzuelo A, Galisteo M. Long-term resveratrol administration reduces metabolic disturbances and lowers blood pressure in obese Zucker rats. Biochem Pharmacol 2009;77(6):1053-63.

53. Wong RH, Howe PR, Buckley JD, Coates AM, Kunz I, Berry NM. Acute resveratrol supplementation improves flow-mediated dilatation in overweight/obese individuals with mildly elevated blood pressure. Nutr Metab Cardiovasc Dis 2010. 


\section{Supplementary information}

\section{Supplementary methods}

\section{Analysis of resveratrol and dihydroresveratrol}

To check compliance, the analytics of resveratrol (original and metabolites) were processed in a blinded fashion by a liquid chromatography mass spectrometry (LCMS) system in both the resveratrol- and placebo-supplemented period by DSM Nutritional Products, Ltd. Kaiseraugst, Switzerland. Plasma collected on day 0, 7, $14,21,30$ and during the stay in the respiration chamber (day 29) was processed for the determination of "free" trans-resveratrol and dihydroresveratrol (aglycone) and "total" trans-resveratrol and dihydroresveratrol (aglycone + glucuronide conjugates). After addition of an internal standard and liquid-liquid extraction ("free" analyte) or pre-digesting by $\beta$-glucuronidase followed by liquid-liquid extraction ("total" analyte), the samples were injected in a C18 column. Detection was performed using MS in the SIM mode.

\section{Plasma biochemistry}

In the morning of day 30 -after a standardized overnight fast for 12 hours- and during the postprandial microdialysis test, blood samples were withdrawn for the determination of plasma glucose, insulin, non-esterified fatty acids (NEFA) and triglycerides (TG) levels as described (1). Glycerol and lactate levels during the microdialysis test were analyzed enzymatically on a Cobas Mira automated spectrophotometer. The concentration of leptin and adiponectin in plasma were analyzed by radioimmunoassay using commercial kits (Millipore Corporation, Billerica, MA, USA) in accordance with the manufacturer's instructions. Highsensitivity C-reactive protein was measured with a commercially available kit (Horiba ABX, Montpellier, France). Plasma inflammatory markers (IL-1b, IL-6, IL-8 and TNF- $\alpha$ ) were measured with a commercially available Multi Spot ELISA kit (Meso Scale Discovery, Gaithersburg, MD, USA).

\section{Molecular and protein expression}

Mitochondrial DNA (mtDNA) copy number, the ratio of NADH dehydrogenase subunit one (ND1) to lipoprotein lipase (LPL) (mtDNA/nuclear DNA) was determined as described (1).

Oxidative phosphorylation (OXPHOS), SIRT1, and PGC1 $\alpha$ protein levels were measured in whole muscle by western blotting as an additional reflection of mitochondrial density, as described (2). OXPHOS proteins were detected using a monoclonal antibody cocktail of five monoclonal antibodies directed against the 
different OXPHOS complexes (MS601, MitoSciences, Eugene, OR). SIRT1 was detected using a polyclonal antibody (\#2493 Cell Signaling, Technology, Inc., Beverly MA, USA). PGC1 $\alpha$ was measured using a polyclonal antibody (Santa Cruz, Heidelberg, Germany). Total and the phosphorylated subunit $\alpha$ of AMPK (\#2531 and \#2532 Cell Signalling Technology, Inc., Beverly MA, USA) were detected as described (3).

\section{Postprandial substrate utilization and tissue lipolysis}

In 10 subjects, the lipolytic effects of resveratrol in adipose tissue and skeletal muscle were successfully determined by microdialysis, essentially according to (4). To study the response of tissue lipolysis on a mixed meal, subjects were instructed to consume a liquid meal within 5 minutes. The total energy content of the shake was 2.6 MJ and consisted of $32.6 \mathrm{En} \%$ carbohydrates, $61.2 \mathrm{En} \%$ fat and $6.3 \mathrm{En} \%$ protein. Blood samples were taken before ingestion of the liquid test meal $(\mathrm{t}=-30$ and $0 \mathrm{~min}$ ) and for $6 \mathrm{~h}$ after meal ingestion at $\mathrm{t}=30,60,90,120,180,240,300$ and $360 \mathrm{~min}$ to determine glycerol, glucose, pyruvate and lactate in the plasma. Microdialysate was collected from the probes in $30 \mathrm{~min}$ fractions during the baseline period and during the early postprandial period (0-120 min) and at $1 \mathrm{~h}$ fractions during the last $4 \mathrm{~h}$ postprandially $(120-360 \mathrm{~min})$ to determine glycerol, glucose, pyruvate and lactate levels. Microdialysates were measured by Pronexus Analytical AB (Stockholm, Sweden) on a CMA 600 Microdialysis Analyzer (CMA Microdialysis $A B$, Solna, Sweden). Energy expenditure and substrate utilization were measured, before and for $6 \mathrm{~h}$ after ingestion of the liquid test meal, using a ventilated hood system that analyses gasses every 15 seconds (Omnical, Maastricht University, The Netherlands) (5). Metabolic rate was calculated from $\mathrm{VO}_{2}(\mathrm{~L} / \mathrm{min})$ and $\mathrm{VCO}_{2}(\mathrm{~L} / \mathrm{min})$ according to the equations of Frayn (6). Nitrogen excretion was calculated based on the assumption that protein oxidation represents $\sim 15 \%$ of total energy expenditure. Energy expenditure was calculated using the formula of Weir (7).

\section{Microarray}

Total RNA was prepared from human muscle using Trizol reagent (Invitrogen, Breda, The Netherlands), treated with DNase and purified on columns using the RNeasy Mini Kit (Qiagen, Venlo, The Netherlands). RNA quantity and quality was assessed spectrophotometrically (ND-1000, NanoDrop Technologies, Wilmington, USA) and with 6000 Nano chips (Bioanalyzer 2100; Agilent, Amstelveen, The Netherlands), respectively.

The Ambion WT Expression kit (Life Technologies, P/N 4411974) and the Affymetrix GeneChip WT Terminal Labeling kit (Affymetrix, Santa Clara, CA; P/N 900671) were used for the preparation of labeled cDNA from 100ng of total RNA. 
Labeled samples were hybridized on Affymetrix GeneChip human Gene 1.1 ST arrays, provided in plate format. Hybridization, washing and scanning of the array plates was performed on an Affymetrix GeneTitan Instrument, according to the manufacturer's recommendations. Detailed protocols can be found in the Affymetrix WT Terminal Labeling and Hybridization User Manual (P/N 702808 revision 4), and are also available upon request.

Quality control of the datasets obtained from the scanned Affymetrix arrays was performed using Bioconductor (8) packages integrated in an on-line pipeline (9). Various advanced quality metrics, diagnostic plots, pseudo-images and classification methods were applied for selection of ascertain only excellent quality arrays were used in the subsequent analyses (10). An extensive description of the applied criteria is available upon request.

The more than 803000 probes on the Human Gene 1.1 ST array were redefined according to Dai et al. (11) utilizing current genome information, yielding 19738 unique genes. Probes were reorganized based on the Entrez Gene database, build 37, version 2 (remapped CDF v14).

Normalized expression estimates were obtained from the raw intensity values using the robust multiarray analysis (RMA) preprocessing algorithm (12). All genes represented on the array were considered for the unbiased Gene Set Enrichment Analysis (GSEA) (13).

GSEA was run using 1000 permutations per gene set. Unsupervised hierarchical clustering was performed using complete linkage and Pearson rank correlation distance on the normalized transcripts using software implemented in Genepattern (http://www.broadinstitute.org/cancer/software/genepattern/) $(14,15)$. The z-score was calculated by subtracting the mean expression value for each transcript from each of the values and then dividing the resulting values by the standard deviation. Color in the heat-maps reflects the relative transcript abundance level with red being higher and blue lower than the mean transcript abundance value. Transcript ordering is determined as in hierarchical clustering using the distance function 1correlation. Mouse microarray data (16) were reanalysed using KEGG pathway gene sets in the GSEA software (Broad institute) (17). 


\section{Supplementary Tables and Figures}

Table S1. Blood safety parameters.

\begin{tabular}{|c|c|c|c|}
\hline & Placebo & Resveratrol & p-value \\
\hline Erythrocytes $\left(10^{12} / \mathrm{l}\right)$ & $4.85 \pm 0.09$ & $4.81 \pm 0.09$ & 0.52 \\
\hline Hemoglobin (mmol/l) & $9.07 \pm 0.17$ & $8.95 \pm 0.15$ & 0.17 \\
\hline Hematocrit (\%) & $44.00 \pm 1.00$ & $43.00 \pm 1.00$ & 0.35 \\
\hline $\operatorname{MCV}(\mathrm{fl})$ & $90.18 \pm 1.73$ & $89.73 \pm 1.72$ & 0.10 \\
\hline $\mathrm{MCHC}(\mathrm{mmol} / \mathrm{l})$ & $20.77 \pm 0.10$ & $20.81 \pm 0.11$ & 0.79 \\
\hline Trombocytes $\left(10^{9} / \mathrm{l}\right)$ & $237.55 \pm 11.88$ & $228.90 \pm 10.60$ & 0.10 \\
\hline RDW (\%) & $13.25 \pm 0.15$ & $13.48 \pm 0.26$ & 0.40 \\
\hline Leucocytes $\left(10^{9} / \mathrm{I}\right)$ & $7.03 \pm 0.44$ & $6.48 \pm 0.39$ & 0.03 \\
\hline PT (sec) & $10.62 \pm 0.06$ & $10.58 \pm 0.09$ & 0.65 \\
\hline aPTT (sec) & $29.00 \pm 0.83$ & $30.63 \pm 1.88$ & 0.48 \\
\hline INR & $1.00 \pm 0.01$ & $1.00 \pm 0.01$ & 0.90 \\
\hline Sodium (mmol/l) & $140.18 \pm 0.35$ & $139.64 \pm 0.58$ & 0.40 \\
\hline Potassium (mmol/l) & $4.26 \pm 0.07$ & $4.18 \pm 0.07$ & 0.57 \\
\hline Phosphate (mmol/l) & $0.88 \pm 0.03$ & $0.92 \pm 0.04$ & 0.08 \\
\hline Chloride (mmol/l) & $106.36 \pm 0.94$ & $106.18 \pm 0.60$ & 0.83 \\
\hline Urea (mmol/l) & $4.50 \pm 0.24$ & $4.37 \pm 0.21$ & 0.15 \\
\hline Creatinine $(\mu \mathrm{mol} / \mathrm{l})$ & $76.10 \pm 3.83$ & $75.82 \pm 3.28$ & 0.83 \\
\hline Alkaline phosphatase (U/I) & $79.00 \pm 4.11$ & $76.55 \pm 3.81$ & 0.30 \\
\hline Gamma GT (U/I) & $29.60 \pm 3.71$ & $28.00 \pm 2.61$ & 0.44 \\
\hline ASAT (U/I) & $19.00 \pm 1.46$ & $18.91 \pm 1.11$ & 0.93 \\
\hline ALAT (U/I) & $31.91 \pm 2.21$ & $28.09 \pm 1.54$ & 0.02 \\
\hline Bilirubin $(\mu \mathrm{mol} / \mathrm{l})$ & $14.25 \pm 1.00$ & $13.86 \pm 1.07$ & 0.42 \\
\hline Total protein (g/l) & $66.55 \pm 1.24$ & $65.39 \pm 0.80$ & 0.14 \\
\hline Albumin (g/l) & $38.02 \pm 0.79$ & $37.33 \pm 0.55$ & 0.18 \\
\hline
\end{tabular}

Blood of the subjects was analyzed for several clinical chemistry, hematology and coagulation markers in order to confirm that resveratrol supplementation did not cause adverse effects. Values on day 30 . Values are given as means $\pm \operatorname{SEM}(n=11)$. 
Table S2. Time course RQ and EE.

\begin{tabular}{|c|c|c|c|c|c|c|c|}
\hline \multirow[b]{2}{*}{ Time (h:min) } & \multirow[b]{2}{*}{ Activity } & \multicolumn{3}{|c|}{ Resveratrol } & \multicolumn{3}{|c|}{ Resveratrol } \\
\hline & & \multicolumn{2}{|c|}{$\mathbf{R Q}$} & \multirow{2}{*}{$\begin{array}{c}\begin{array}{c}\text { P- } \\
\text { value }\end{array} \\
0.09\end{array}$} & \multicolumn{2}{|c|}{$E E(M J / d)$} & $\begin{array}{c}\text { P- } \\
\text { value }\end{array}$ \\
\hline- & SMR & $0.87 \pm 0.01$ & $0.89 \pm 0.01$ & & $8.09 \pm 0.24$ & $7.75 \pm 0.23$ & 0.007 \\
\hline $9.00-10.00$ & breakfast & $0.87 \pm 0.01$ & $0.89 \pm 0.01$ & 0.17 & $11.86 \pm 0.57$ & $11.11 \pm 0.46$ & 0.05 \\
\hline $10.00-13.00$ & stepping + snack & $0.91 \pm 0.01$ & $0.94 \pm 0.01$ & 0.02 & $14.40 \pm 0.32$ & $13.86 \pm 0.32$ & 0.07 \\
\hline $13.00-15.30$ & lunch & $0.89 \pm 0.01$ & $0.89 \pm 0.01$ & 0.44 & $11.75 \pm 0.44$ & $11.65 \pm 0.47$ & 0.44 \\
\hline $15.30-18.00$ & stepping + snack & $0.90 \pm 0.01$ & $0.93 \pm 0.01$ & 0.01 & $14.85 \pm 0.31$ & $14.55 \pm 0.25$ & 0.24 \\
\hline $18.00-20.00$ & diner & $0.89 \pm 0.01$ & $0.90 \pm 0.01$ & 0.35 & $12.62 \pm 0.39$ & $12.26 \pm 0.43$ & 0.16 \\
\hline $20.00-24.00$ & stepping + snack & $0.91 \pm 0.01$ & $0.92 \pm 0.01$ & 0.33 & $13.16 \pm 0.21$ & $13.49 \pm 0.23$ & 0.27 \\
\hline- & SMR & $0.88 \pm 0.01$ & $0.90 \pm 0.01$ & 0.21 & $8.06 \pm 0.22$ & $7.90 \pm 0.18$ & 0.06 \\
\hline
\end{tabular}

Detailed time course of $R Q$ and energy $E E$ over the last $24 \mathrm{~h}$ of resveratrol or placebo supplementation. Values on day 30. Values are given as means $\pm \operatorname{SEM}(n=10)$.

Table S3. Comparison of calorie restriction with resveratrol.

\begin{tabular}{lcccc}
\hline & \multicolumn{2}{c}{ Calorie restriction } & \multicolumn{2}{c}{ Resveratrol } \\
\hline & Rodents & Humans & Rodents & Humans \\
\hline Body weight & $\downarrow$ & $\downarrow$ & $\neq \downarrow$ & $\neq$ \\
Insulin & $\downarrow$ & $\downarrow$ & $\downarrow$ & $\downarrow$ \\
Energy expenditure & & & & \\
$\quad$ Total & $\downarrow$ & $\downarrow$ & $\uparrow$ & $\neq$ \\
$\quad$ Postprandial & $\downarrow$ & $\downarrow$ & $?$ & $\downarrow$ \\
$\quad$ Sendentary/Sleep & $\downarrow$ & $\downarrow$ & $?$ & $?$ \\
Fat mass and fat-free mass & $\downarrow$ & $\downarrow$ & $\downarrow$ & $\downarrow$ \\
Liver fat & $\downarrow$ & $\downarrow$ & $\downarrow$ & $\uparrow$ \\
Intramyocellular lipids & $\downarrow$ & $\downarrow$ & $\downarrow$ & $\uparrow$ \\
Insulin sensitivity & $\uparrow$ & $\uparrow$ & $\uparrow$ & $\downarrow$ \\
Inflammation markers & $\downarrow$ & $\downarrow$ & $\downarrow$ & $\uparrow$ \\
Mitochondrial efficiency & $\uparrow$ & $\uparrow$ & $\uparrow$ & $\downarrow$ \\
\hline
\end{tabular}

Comparison of chronic calorie restriction in rodents and overweight humans to the effects of 30 days of resveratrol supplementation in obese humans on markers of ageing. The measured effects of calorie restriction and resveratrol on the above markers of ageing were obtained from the following references for rodents and for humans: $(16,18-99)$. $\downarrow$ decrease, $\uparrow$ increase, $\neq$ similar, ? currently unknown 


\section{Chapter 3}

\section{FIGURE S1}

A

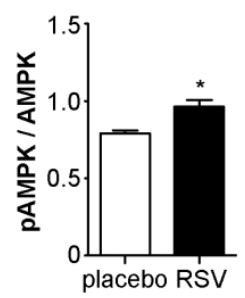

B

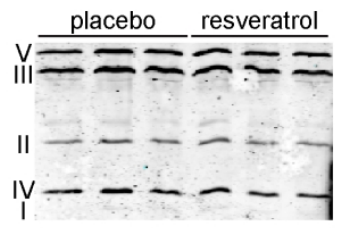

C

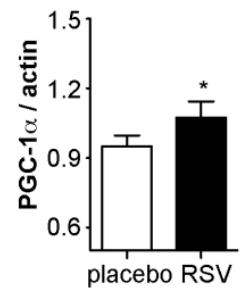

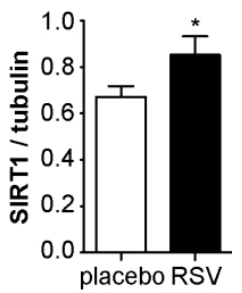

Figure S1. Western blot analysis. (A) Quantitative analysis of the effect of resveratrol (RSV) on the phosphorylation of AMPK on $\mathrm{Thr}^{172}$ of the $\alpha$-subunit in vastus lateralis muscle. Data are presented as the ratio of pAMPK/AMPK $(n=9)$. (B) Representative Western blot for the individual complexes of the electron transport chain ( $n=11$, a subset of 3 subjects is shown). (C) Quantitative analysis of the Western blots of PGC1 $\alpha$ and SIRT1. Data are normalized for the loading control, actin for PGC1 $\alpha$ and tubulin for SIRT1. Values are given as means \pm SEM ( $n=11$ for PGC1 $\alpha, n=8$ for SIRT1), ${ }^{*} p<0.05$. 


\section{Supplementary references}

1. Phielix E, Schrauwen-Hinderling VB, Mensink M, et al. Lower intrinsic ADP-stimulated mitochondrial respiration underlies in vivo mitochondrial dysfunction in muscle of male type 2 diabetic patients. Diabetes 2008;57(11):2943-9.

2. Timmers S, de Vogel-van den Bosch J, Hesselink MK, et al. Paradoxical increase in TAG and DAG content parallel the insulin sensitizing effect of unilateral DGAT1 overexpression in rat skeletal muscle. PLoS One 2011;6(1):e14503.

3. Feige JN, Lagouge M, Canto C, et al. Specific SIRT1 activation mimics low energy levels and protects against diet-induced metabolic disorders by enhancing fat oxidation. Cell Metab 2008;8(5):347-58.

4. Goossens GH, Blaak EE, Saris WH, van Baak MA. Angiotensin II-induced effects on adipose and skeletal muscle tissue blood flow and lipolysis in normal-weight and obese subjects. J Clin Endocrinol Metab 2004;89(6):2690-6.

5. Schoffelen PF, Westerterp KR, Saris WH, Ten Hoor F. A dual-respiration chamber system with automated calibration. J Appl Physiol 1997;83(6):2064-72.

6. Frayn KN. Calculation of substrate oxidation rates in vivo from gaseous exchange. J Appl Physiol 1983;55(2):628-34.

7. Weir JB. New methods for calculating metabolic rate with special reference to protein metabolism. J Physiol 1949;109(1-2):1-9.

8. Gentleman RC, Carey VJ, Bates DM, et al. Bioconductor: open software development for computational biology and bioinformatics. Genome Biol 2004;5(10):R80.

9. Lin K, Kools $\mathrm{H}$, de Groot PJ, et al. MADMAX - Management and analysis database for multiple omics experiments. J Integr Bioinform 2011;8(2):160.

10. Heber S, Sick B. Quality assessment of Affymetrix GeneChip data. Omics 2006;10(3):358-68.

11. Dai $M$, Wang $P$, Boyd $A D$, et al. Evolving gene/transcript definitions significantly alter the interpretation of GeneChip data. Nucleic Acids Res 2005;33(20):e175.

12. Irizarry RA, Hobbs B, Collin F, et al. Exploration, normalization, and summaries of high density oligonucleotide array probe level data. Biostatistics 2003;4(2):249-64.

13. Subramanian A, Tamayo P, Mootha VK, et al. Gene set enrichment analysis: a knowledgebased approach for interpreting genome-wide expression profiles. Proc Natl Acad Sci U S A 2005;102(43):15545-50.

14. de Hoon MJ, Imoto S, Nolan J, Miyano S. Open source clustering software. Bioinformatics 2004;20(9):1453-4.

15. Reich M, Liefeld T, Gould J, Lerner J, Tamayo P, Mesirov JP. GenePattern 2.0. Nat Genet 2006;38(5):500-1.

16. Lagouge M, Argmann C, Gerhart-Hines Z, et al. Resveratrol improves mitochondrial function and protects against metabolic disease by activating SIRT1 and PGC-1alpha. Cell 2006;127(6):1109-22.

17. Subramanian A, Tamayo P, Mootha VK, et al. Gene set enrichment analysis: a knowledgebased approach for interpreting genome-wide expression profiles. Proc Natl Acad Sci U S A 2005;102(43):15545-50.

18. Ballor DL. Effect of dietary restriction and/or exercise on 23-h metabolic rate and body composition in female rats. J Appl Physiol 1991;71(3):801-6.

19. Havel PJ. Control of energy homeostasis and insulin action by adipocyte hormones: leptin, acylation stimulating protein, and adiponectin. Curr Opin Lipidol 2002;13(1):51-9.

20. Sohal RS, Weindruch R. Oxidative stress, caloric restriction, and aging. Science 1996;273(5271):59-63.

21. Meites J. Evidence that underfeeding acts via the neuroendocrine system to influence aging processes. Prog Clin Biol Res 1989;287:169-80.

22. Lee CK, Klopp RG, Weindruch R, Prolla TA. Gene expression profile of aging and its retardation by caloric restriction. Science 1999;285(5432):1390-3.

23. Russell JC, Epling WF, Pierce D, Amy RM, Boer DP. Induction of voluntary prolonged running by rats. J Appl Physiol 1987;63(6):2549-53.

24. Duffy PH, Feuers R, Nakamura KD, Leakey J, Hart RW. Effect of chronic caloric restriction on the synchronization of various physiological measures in old female Fischer 344 rats. Chronobiol Int 1990;7(2):113-24.

25. Corton JC, Apte U, Anderson SP, et al. Mimetics of caloric restriction include agonists of lipidactivated nuclear receptors. J Biol Chem 2004;279(44):46204-12. 
26. Dhahbi JM, Mote PL, Fahy GM, Spindler SR. Identification of potential caloric restriction mimetics by microarray profiling. Physiol Genomics 2005;23(3):343-50.

27. Dhahbi JM, Tsuchiya T, Kim HJ, Mote PL, Spindler SR. Gene expression and physiologic responses of the heart to the initiation and withdrawal of caloric restriction. J Gerontol A Biol Sci Med Sci 2006;61(3):218-31.

28. Edwards MG, Anderson RM, Yuan M, Kendziorski CM, Weindruch R, Prolla TA. Gene expression profiling of aging reveals activation of a p53-mediated transcriptional program. BMC Genomics 2007;8:80.

29. Fu C, Hickey M, Morrison M, McCarter R, Han ES. Tissue specific and non-specific changes in gene expression by aging and by early stage CR. Mech Ageing Dev 2006;127(12):905-16.

30. Higami Y, Pugh TD, Page GP, Allison DB, Prolla TA, Weindruch R. Adipose tissue energy metabolism: altered gene expression profile of mice subjected to long-term caloric restriction. FASEB J 2004;18(2):415-7.

31. Lee CK, Allison DB, Brand J, Weindruch R, Prolla TA. Transcriptional profiles associated with aging and middle age-onset caloric restriction in mouse hearts. Proc Natl Acad Sci U S A 2002;99(23):14988-93.

32. Selman C, Kerrison ND, Cooray A, et al. Coordinated multitissue transcriptional and plasma metabonomic profiles following acute caloric restriction in mice. Physiol Genomics 2006;27(3):187-200.

33. Tsuchiya T, Dhahbi JM, Cui X, Mote PL, Bartke A, Spindler SR. Additive regulation of hepatic gene expression by dwarfism and caloric restriction. Physiol Genomics 2004;17(3):307-15.

34. Barzilai N, Banerjee S, Hawkins M, Chen W, Rossetti L. Caloric restriction reverses hepatic insulin resistance in aging rats by decreasing visceral fat. J Clin Invest 1998;101(7):1353-61.

35. Bertrand HA, Lynd FT, Masoro EJ, Yu BP. Changes in adipose mass and cellularity through the adult life of rats fed ad libitum or a life-prolonging restricted diet. J Gerontol 1980;35(6):827-35.

36. Barger $\mathrm{JL}$, Walford RL, Weindruch $\mathrm{R}$. The retardation of aging by caloric restriction: its significance in the transgenic era. Exp Gerontol 2003;38(11-12):1343-51.

37. Goodrick CL, Ingram DK, Reynolds MA, Freeman JR, Cider N. Effects of intermittent feeding upon body weight and lifespan in inbred mice: interaction of genotype and age. Mech Ageing Dev 1990;55(1):69-87.

38. Bruss MD, Khambatta CF, Ruby MA, Aggarwal I, Hellerstein MK. Calorie restriction increases fatty acid synthesis and whole body fat oxidation rates. Am J Physiol Endocrinol Metab 2010;298(1):E108-16.

39. Escriva F, Gavete ML, Fermin Y, et al. Effect of age and moderate food restriction on insulin sensitivity in Wistar rats: role of adiposity. J Endocrinol 2007;194(1):131-41.

40. Wang Z, Al-Regaiey KA, Masternak MM, Bartke A. Adipocytokines and lipid levels in Ames dwarf and calorie-restricted mice. J Gerontol A Biol Sci Med Sci 2006;61(4):323-31.

41. Fan JG, Zhong L, Xu ZJ, et al. Effects of low-calorie diet on steatohepatitis in rats with obesity and hyperlipidemia. World J Gastroenterol 2003;9(9):2045-9.

42. Zheng $\mathrm{Y}$, Zhang W, Pendleton E, et al. Improved insulin sensitivity by calorie restriction is associated with reduction of ERK and P70S6K activities in the liver of obese Zucker rats. $J$ Endocrinol 2009;203(3):337-47.

43. Nadeau KJ, Ehlers LB, Aguirre LE, et al. Exercise training and calorie restriction increase SREBP-1 expression and intramuscular triglyceride in skeletal muscle. Am J Physiol Endocrinol Metab 2006;291(1):E90-8.

44. Drapeau S, Doucet E, Rabasa-Lhoret R, Brochu M, Prud'homme D, Imbeault P. Improvement in insulin sensitivity by weight loss does not affect hyperinsulinemia-mediated reduction in total and high molecular weight adiponectin: a MONET study. Appl Physiol Nutr Metab 2011;36(2):191-200.

45. Lim EL, Hollingsworth KG, Aribisala BS, Chen MJ, Mathers JC, Taylor R. Reversal of type 2 diabetes: normalisation of beta cell function in association with decreased pancreas and liver triacylglycerol. Diabetologia 2011;54(10):2506-14.

46. Labayen I, Ortega FB, Ruiz JR, Lasa A, Simon E, Margareto J. Role of baseline leptin and ghrelin levels on body weight and fat mass changes after an energy-restricted diet intervention in obese women: effects on energy metabolism. J Clin Endocrinol Metab 2011;96(6):E996-1000.

47. Haufe $S$, Engeli $S$, Kast $P$, et al. Randomized comparison of reduced fat and reduced carbohydrate hypocaloric diets on intrahepatic fat in overweight and obese human subjects. Hepatology 2011;53(5):1504-14. 
48. Civitarese AE, Carling S, Heilbronn LK, et al. Calorie restriction increases muscle mitochondrial biogenesis in healthy humans. PLoS Med 2007;4(3):e76.

49. Das SK, Gilhooly CH, Golden JK, et al. Long-term effects of 2 energy-restricted diets differing in glycemic load on dietary adherence, body composition, and metabolism in CALERIE: a 1-y randomized controlled trial. Am J Clin Nutr 2007;85(4):1023-30.

50. Dengel DR, Pratley RE, Hagberg JM, Rogus EM, Goldberg AP. Distinct effects of aerobic exercise training and weight loss on glucose homeostasis in obese sedentary men. J Appl Physiol 1996;81(1):318-25.

51. Heilbronn LK, de Jonge L, Frisard MI, et al. Effect of 6-month calorie restriction on biomarkers of longevity, metabolic adaptation, and oxidative stress in overweight individuals: a randomized controlled trial. JAMA 2006;295(13):1539-48.

52. Larson-Meyer DE, Heilbronn LK, Redman LM, et al. Effect of calorie restriction with or without exercise on insulin sensitivity, beta-cell function, fat cell size, and ectopic lipid in overweight subjects. Diabetes Care 2006;29(6):1337-44.

53. Lefevre M, Redman LM, Heilbronn LK, et al. Caloric restriction alone and with exercise improves CVD risk in healthy non-obese individuals. Atherosclerosis 2009;203(1):206-13.

54. Martin CK, Anton SD, Han H, et al. Examination of cognitive function during six months of calorie restriction: results of a randomized controlled trial. Rejuvenation Res 2007;10(2):17990.

55. Martin CK, Heilbronn LK, de Jonge L, et al. Effect of calorie restriction on resting metabolic rate and spontaneous physical activity. Obesity (Silver Spring) 2007;15(12):2964-73.

56. Niskanen L, Uusitupa M, Sarlund H, Siitonen O, Paljarvi L, Laakso M. The effects of weight loss on insulin sensitivity, skeletal muscle composition and capillary density in obese nondiabetic subjects. Int J Obes Relat Metab Disord 1996;20(2):154-60.

57. Racette SB, Weiss EP, Villareal DT, et al. One year of caloric restriction in humans: feasibility and effects on body composition and abdominal adipose tissue. J Gerontol A Biol Sci Med Sci 2006;61(9):943-50.

58. Redman LM, Heilbronn LK, Martin CK, Alfonso A, Smith SR, Ravussin E. Effect of calorie restriction with or without exercise on body composition and fat distribution. J Clin Endocrinol Metab 2007;92(3):865-72.

59. Walford RL, Harris SB, Gunion MW. The calorically restricted low-fat nutrient-dense diet in Biosphere 2 significantly lowers blood glucose, total leukocyte count, cholesterol, and blood pressure in humans. Proc Natl Acad Sci U S A 1992;89(23):11533-7.

60. Walford RL, Mock D, MacCallum T, Laseter JL. Physiologic changes in humans subjected to severe, selective calorie restriction for two years in biosphere 2: health, aging, and toxicological perspectives. Toxicol Sci 1999;52(2 Suppl):61-5.

61. Weyer C, Walford RL, Harper IT, et al. Energy metabolism after $2 \mathrm{y}$ of energy restriction: the biosphere 2 experiment. Am J Clin Nutr 2000;72(4):946-53.

62. Williamson DA, Martin CK, York-Crowe E, et al. Measurement of dietary restraint: validity tests of four questionnaires. Appetite 2007;48(2):183-92.

63. Fontana L, Meyer TE, Klein S, Holloszy JO. Long-term calorie restriction is highly effective in reducing the risk for atherosclerosis in humans. Proc Natl Acad Sci U S A 2004;101(17):665963.

64. Weiss EP, Racette SB, Villareal DT, et al. Improvements in glucose tolerance and insulin action induced by increasing energy expenditure or decreasing energy intake: a randomized controlled trial. Am J Clin Nutr 2006;84(5):1033-42.

65. Goodpaster BH, Kelley DE, Wing RR, Meier A, Thaete FL. Effects of weight loss on regional fat distribution and insulin sensitivity in obesity. Diabetes 1999;48(4):839-47.

66. Chen LL, Zhang HH, Zheng J, et al. Resveratrol attenuates high-fat diet-induced insulin resistance by influencing skeletal muscle lipid transport and subsarcolemmal mitochondrial beta-oxidation. Metabolism 2011.

67. Baur JA. Resveratrol, sirtuins, and the promise of a DR mimetic. Mech Ageing Dev 2010;131(4):261-9.

68. Ahn J, Cho I, Kim S, Kwon D, Ha T. Dietary resveratrol alters lipid metabolism-related gene expression of mice on an atherogenic diet. J Hepatol 2008;49(6):1019-28.

69. Barger JL, Kayo T, Vann JM, et al. A low dose of dietary resveratrol partially mimics caloric restriction and retards aging parameters in mice. PLoS One 2008;3(6):e2264.

70. Baur JA, Pearson KJ, Price NL, et al. Resveratrol improves health and survival of mice on a high-calorie diet. Nature 2006;444(7117):337-42.

71. Bhat KPL, Kosmeder JW, 2nd, Pezzuto JM. Biological effects of resveratrol. Antioxid Redox Signal 2001;3(6):1041-64. 
72. Pearson $\mathrm{KJ}$, Baur JA, Lewis $\mathrm{KN}$, et al. Resveratrol delays age-related deterioration and mimics transcriptional aspects of dietary restriction without extending life span. Cell Metab 2008;8(2):157-68.

73. Ramadori G, Gautron L, Fujikawa T, Vianna CR, Elmquist JK, Coppari R. Central administration of resveratrol improves diet-induced diabetes. Endocrinology 2009;150(12):5326-33.

74. Rivera L, Moron R, Zarzuelo A, Galisteo M. Long-term resveratrol administration reduces metabolic disturbances and lowers blood pressure in obese Zucker rats. Biochem Pharmacol 2009;77(6):1053-63.

75. Su HC, Hung LM, Chen JK. Resveratrol, a red wine antioxidant, possesses an insulin-like effect in streptozotocin-induced diabetic rats. Am J Physiol Endocrinol Metab 2006;290(6):E1339-46.

76. Sun $\mathrm{C}$, Zhang $\mathrm{F}, \mathrm{Ge} X$, et al. SIRT1 improves insulin sensitivity under insulin-resistant conditions by repressing PTP1B. Cell Metab 2007;6(4):307-19.

77. Vang O, Ahmad N, Baile CA, et al. What is new for an old molecule? Systematic review and recommendations on the use of resveratrol. PLoS One 2011;6(6):e19881.

78. Goodpaster BH, Theriault R, Watkins SC, Kelley DE. Intramuscular lipid content is increased in obesity and decreased by weight loss. Metabolism 2000;49(4):467-72.

79. Petersen KF, Dufour S, Befroy D, Lehrke M, Hendler RE, Shulman Gl. Reversal of nonalcoholic hepatic steatosis, hepatic insulin resistance, and hyperglycemia by moderate weight reduction in patients with type 2 diabetes. Diabetes 2005;54(3):603-8.

80. Deng JY, Hsieh PS, Huang JP, Lu LS, Hung LM. Activation of estrogen receptor is crucial for resveratrol-stimulating muscular glucose uptake via both insulin-dependent and -independent pathways. Diabetes 2008;57(7):1814-23.

81. Shang J, Chen LL, Xiao FX, Sun H, Ding HC, Xiao H. Resveratrol improves non-alcoholic fatty liver disease by activating AMP-activated protein kinase. Acta Pharmacol Sin 2008;29(6):698-706.

82. Palsamy P, Subramanian S. Resveratrol, a natural phytoalexin, normalizes hyperglycemia in streptozotocin-nicotinamide induced experimental diabetic rats. Biomed Pharmacother 2008;62(9):598-605.

83. Gonzalez-Rodriguez A, Mas Gutierrez JA, Sanz-Gonzalez S, Ros M, Burks DJ, Valverde AM. Inhibition of PTP1B restores IRS1-mediated hepatic insulin signaling in IRS2-deficient mice. Diabetes 2010;59(3):588-99.

84. $U \mathrm{~m} \mathrm{JH}$, Park SJ, Kang $\mathrm{H}$, et al. AMP-activated protein kinase-deficient mice are resistant to the metabolic effects of resveratrol. Diabetes 2010;59(3):554-63.

85. Rocha KK, Souza GA, Ebaid GX, Seiva FR, Cataneo AC, Novelli EL. Resveratrol toxicity: effects on risk factors for atherosclerosis and hepatic oxidative stress in standard and high-fat diets. Food Chem Toxicol 2009;47(6):1362-7.

86. Huang JP, Huang SS, Deng JY, Chang CC, Day YJ, Hung LM. Insulin and resveratrol act synergistically, preventing cardiac dysfunction in diabetes, but the advantage of resveratrol in diabetics with acute heart attack is antagonized by insulin. Free Radic Biol Med 2010;49(11):1710-21.

87. Thirunavukkarasu M, Penumathsa SV, Koneru S, et al. Resveratrol alleviates cardiac dysfunction in streptozotocin-induced diabetes: Role of nitric oxide, thioredoxin, and heme oxygenase. Free Radic Biol Med 2007;43(5):720-9.

88. Milne JC, Lambert PD, Schenk S, et al. Small molecule activators of SIRT1 as therapeutics for the treatment of type 2 diabetes. Nature 2007;450(7170):712-6.

89. Kim YH, Kim YS, Kang SS, Cho GJ, Choi WS. Resveratrol inhibits neuronal apoptosis and elevated $\mathrm{Ca} 2+/$ calmodulin-dependent protein kinase II activity in diabetic mouse retina. Diabetes 2010;59(7):1825-35.

90. Macarulla MT, Alberdi G, Gomez S, et al. Effects of different doses of resveratrol on body fat and serum parameters in rats fed a hypercaloric diet. J Physiol Biochem 2009;65(4):369-76.

91. Aubin MC, Lajoie C, Clement R, Gosselin H, Calderone A, Perrault LP. Female rats fed a high-fat diet were associated with vascular dysfunction and cardiac fibrosis in the absence of overt obesity and hyperlipidemia: therapeutic potential of resveratrol. J Pharmacol Exp Ther 2008;325(3):961-8.

92. Bujanda L, Hijona E, Larzabal M, et al. Resveratrol inhibits nonalcoholic fatty liver disease in rats. BMC Gastroenterol 2008;8:40.

93. Kumar A, Sharma SS. NF-kappaB inhibitory action of resveratrol: a probable mechanism of neuroprotection in experimental diabetic neuropathy. Biochem Biophys Res Commun 2010;394(2):360-5. 
94. Palsamy $\mathrm{P}$, Subramanian S. Ameliorative potential of resveratrol on proinflammatory cytokines, hyperglycemia mediated oxidative stress, and pancreatic beta-cell dysfunction in streptozotocin-nicotinamide-induced diabetic rats. J Cell Physiol 2010;224(2):423-32.

95. Tunali-Akbay T, Sehirli O, Ercan F, Sener G. Resveratrol protects against methotrexateinduced hepatic injury in rats. J Pharm Pharm Sci 2010;13(2):303-10.

96. Zhang H, Morgan B, Potter BJ, et al. Resveratrol improves left ventricular diastolic relaxation in type 2 diabetes by inhibiting oxidative/nitrative stress: in vivo demonstration with magnetic resonance imaging. Am J Physiol Heart Circ Physiol 2010;299(4):H985-94.

97. Sharma S, Chopra K, Kulkarni SK. Effect of insulin and its combination with resveratrol or curcumin in attenuation of diabetic neuropathic pain: participation of nitric oxide and TNFalpha. Phytother Res 2007;21(3):278-83.

98. Kim JY, Kim DH, Choi J, et al. Changes in lipid distribution during aging and its modulation by calorie restriction. Age (Dordr) 2009;31(2):127-42.

99. Rahman M, Halade GV, Bhattacharya A, Fernandes G. The fat-1 transgene in mice increases antioxidant potential, reduces pro-inflammatory cytokine levels, and enhances PPAR-gamma and SIRT-1 expression on a calorie restricted diet. Oxid Med Cell Longev 2009;2(5):307-16. 


\title{
4
}

\section{The effects of $\mathbf{3 0}$ days resveratrol supplementation on adipose tissue morphology and gene expression patterns in obese men}

\author{
Ellen Konings* \\ Silvie Timmers* \\ Mark V. Boekschoten \\ Gijs H. Goossens \\ Johan W. Jocken \\ Lydia A. Afman \\ Michael Müller \\ Patrick Schrauwen \\ Edwin C. Mariman \\ Ellen E. Blaak
}

* These authors contributed equally 


\section{Abstract}

Background: Polyphenolic compounds, such as resveratrol have recently received widespread interest due to their ability to mimic effects of calorie restriction.

Aim: The objective of the present study was to gain more insight into the effects of 30 days resveratrol supplementation on adipose tissue morphology and underlying processes.

Methods: Eleven healthy obese men were supplemented with placebo and resveratrol for 30 days ( $150 \mathrm{mg} /$ day), separated by a 4-week washout period in a double-blind randomized crossover design. A postprandial abdominal subcutaneous adipose tissue biopsy was collected to assess adipose tissue morphology and gene expression using microarray analysis.

Results: Resveratrol significantly decreased adipocyte size, with a shift towards a reduction in the proportion of large and very large adipocytes and an increase in small adipocytes. Microarray analysis revealed downregulation of Wnt, Notch and BMP/TGF- $\beta$ signaling pathways and upregulation of pathways involved in cell cycle regulation after resveratrol supplementation, suggesting enhanced adipogenesis. Furthermore, the lysosomal/phagosomal pathway and the transcription factor EB (TFEB) were upregulated reflecting an alternative pathway of lipid breakdown by autophagy.

Conclusion: These data suggest that adipose tissue is an important target tissue for the effects of resveratrol in humans, but further research is necessary to investigate whether it translates into an improved adipose tissue function. 


\section{Introduction}

Obesity is reaching epidemic proportions and is associated with insulin resistance and an increased risk for type 2 diabetes (1). Polyphenolic compounds, such as resveratrol are currently an area of intense investigation due to their ability to mimic effects of calorie restriction. Resveratrol (trans-3,5,4'-trihydroxystilbene) is synthesized in a large number of plant species including some components of the human diet, such as mulberries, peanuts, grapes, and red wines. Pronounced effects of resveratrol have been reported on lipolysis, adipogenesis and inflammation in isolated adipocytes and on adipose tissue of murine animals (2). These effects may possibly modulate adipose tissue function and contribute to a reduced body fat accumulation and improved insulin sensitivity $(3,4)$. However, data on the effects of resveratrol on adipose tissue function in the human in vivo situation are lacking. Previously, we demonstrated beneficial effects of 30 days resveratrol supplementation on the whole body metabolic profile, mimicking effects of calorie restriction, in healthy obese men (5).

The general aim of the present work was to gain more insight into the effects of 30 days resveratrol supplementation on adipose tissue morphology and to investigate the underlying mechanisms using microarray analysis in healthy obese men.

\section{Methods}

\section{Study design}

The current work was conducted as part of a previously published randomized double-blind crossover study (5). Eleven obese, healthy men participated in two experimental trials for 30 days: a placebo and a resVida $(150 \mathrm{mg} /$ day transresveratrol [99.9\%; DSM Nutritional Products, Ltd.]) condition, with a 4-week washout period. At the end of both intervention periods (resveratrol and placebo), a subcutaneous adipose tissue biopsy was taken 6 hours after ingestion of a high-fat liquid test meal (5). The original study protocol was reviewed and approved by the Medical Ethical Committee of Maastricht University Medical Center and written informed consent was obtained before study participation.

\section{Abdominal subcutaneous adipocyte size}

Processing and analysis of abdominal subcutaneous adipose tissue biopsies for adipocyte size have been described in detail previously (6). Briefly, an adipose tissue needle biopsy $(\sim 1 \mathrm{~g})$ was collected $6-8 \mathrm{~cm}$ lateral from the umbilicus under local anaesthesia. Part of the adipose tissue was fixed overnight in $4 \%$ paraformaldehyde and embedded in paraffin for histological sections $(8 \mu \mathrm{m})$, 
whereas the other part of the biopsy was snap frozen in liquid nitrogen and stored at $-80^{\circ} \mathrm{C}$. Sections were stained with hematoxylin and eosin (HE). Digital images were captured and computerized morphometric analysis of individual adipocytes was performed in a blinded fashion.

\section{Adipose tissue gene expression}

\section{Microarray processing}

Total RNA was extracted from frozen adipose tissue specimens using TRIzol reagent (Invitrogen, Breda, the Netherlands) and purified on columns using the Qiagen RNeasy Micro Kit (Qiagen, Venlo, Netherlands). Total RNA (100 $\mathrm{ng} /$ sample) was labeled by Whole Transcript Sense Target Assay and hybridized to human whole-genome Affymetrix Gene 1.1 ST arrays targeting 19,793 unique genes (Affymetrix, Santa Clara, CA).

\section{Microarray data analysis}

Quality control and data analysis has been described in detail previously (5). Individual genes were defined as changed when comparison of the normalized signal intensities showed a $P$ value $<0.05$ in a 2 - tailed paired intensity-based moderated t-statistics (IBMT) (PMID:17177995). These analysis were performed within MADMAX system (PMID:21778530). Further functional data analysis was performed on the filtered dataset with Gene Set Enrichment Analysis (http://www.broad.mit.edu/gsea/) and on the differentially expressed genes with Ingenuity Pathway Analysis (June 2012, Ingenuity Systems, Redwood City, CA). Array data have been submitted to the Gene Expression Omnibus GSE42432.

\section{Statistics}

Kolmogorov-Smirnov normality test was performed to evaluate normality distribution. Student's paired t test was used to compare placebo and resveratrol supplementation in normally distributed data; otherwise, Wilcoxon signed rank test was used. A P value $<0.05$ was considered statistically significant. Data are reported as mean \pm SEM. Statistical analyses were performed using the statistical program SPSS 16.0. 


\section{Results}

\section{Subject characteristics}

Eleven obese male volunteers aged 40-65 years and BMI between $28-36 \mathrm{~kg} / \mathrm{m}^{2}$ participated in this study. As previously reported (5), 30 days of resveratrol supplementation resulted in reduced plasma glucose $(5.06 \pm 0.13 \mathrm{mmol} / \mathrm{lvs} .5 .28 \pm$ $0.15 \mathrm{mmol} / \mathrm{l}, \mathrm{P}=0.05)$ and insulin concentrations $(10.31 \pm 1.25 \mu \mathrm{U} / \mathrm{l}$ vs. $11.94 \pm 1.11$ $\mu \mathrm{U} / \mathrm{l}, \mathrm{P}=0.04)$ and a lower HOMA-IR compared with placebo $(2.43 \pm 0.24$ vs. 2.80 $\pm 0.20, P=0.03$ ), suggesting improved insulin sensitivity.

\section{Resveratrol decreased abdominal subcutaneous adipocyte size}

Mean adipocyte size was decreased after 30 days resveratrol $(65.0 \pm 4.4 \mu \mathrm{m})$ compared with placebo $(74.7 \pm 3.5 \mu \mathrm{m})$ (Figure 1C). This was attributed by a shift towards a lower proportion of large $(70-89 \mathrm{~m})$ and very large adipocytes $(>90 \mu \mathrm{m})$ and an increased proportion of small adipocytes $(<50 \mu \mathrm{m})$ (Figure 1D). A representative image is shown in Figure 1A and 1B.

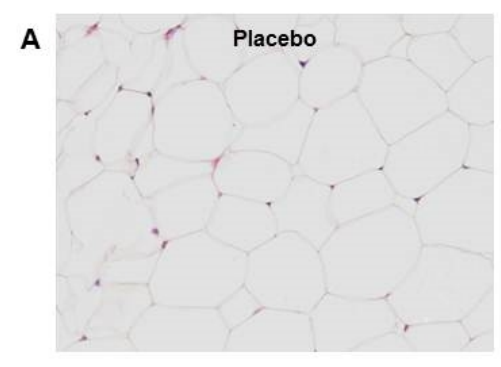

C

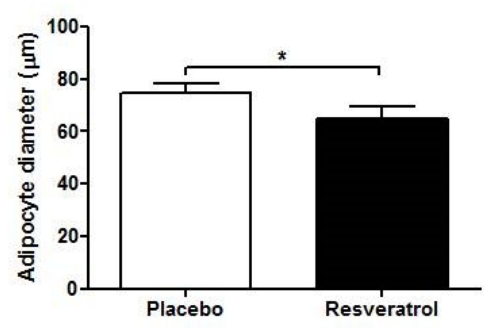

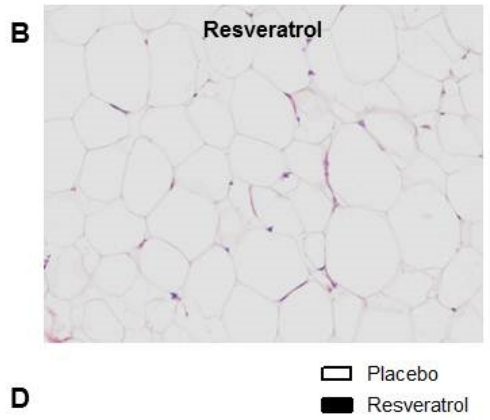

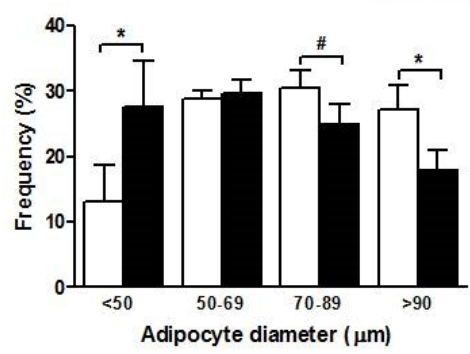

Figure 1. Adipocyte size measurement after placebo and resveratrol supplementation. Representative adipose tissue sections after placebo (A) and resveratrol (B) supplementation (20x magnification). (C) Mean adipocyte diameter. (D) Adipocyte size distribution. $N=8$. Values are means \pm SEM. \# $p<0.1,{ }^{*} p<0.05$. 


\section{Microarray analysis}

From the 19,793 genes on the array, 12,676 genes were expressed in adipose tissue. Resveratrol supplementation resulted in changed expression of 582 genes, of which 290 were upregulated and 292 were downregulated.

\section{Gene Set Enrichment Analysis}

Gene sets that were downregulated by resveratrol belonged to pathways involved in cell-cell junction, Wnt signaling, angiogenesis, G protein coupled receptors (GPCRs) and Notch signaling (Figure 2A). Gene sets upregulated by resveratrol were associated with lysosome, phagosome, inflammation, glucose/hexose transport and cell cycle (Figure 2B). Upregulated pathways associated with inflammation included interferon signaling, antigen processing and presentation, $T$ cell receptor signaling and NFKB activation.

\section{Ingenuity pathway analysis}

Besides GSEA, we analyzed the differentially expressed genes with Ingenuity Pathway Analysis. The ten most upregulated pathways were involved in the inflammatory processes, fibrosis (including fibronectin and matrix metalloproteinases), cell proliferation (Rac signaling), stillbene biosynthesis and lipid antigen presentation. The ten most downregulated pathways were involved in Notch signaling, Wnt signaling, bone morphogenetic protein (BMP) signaling and transforming growth factor- $\beta$ (TGF- $\beta$ ) signaling (Figure 3). These pathways were comparable with those of GSEA. 

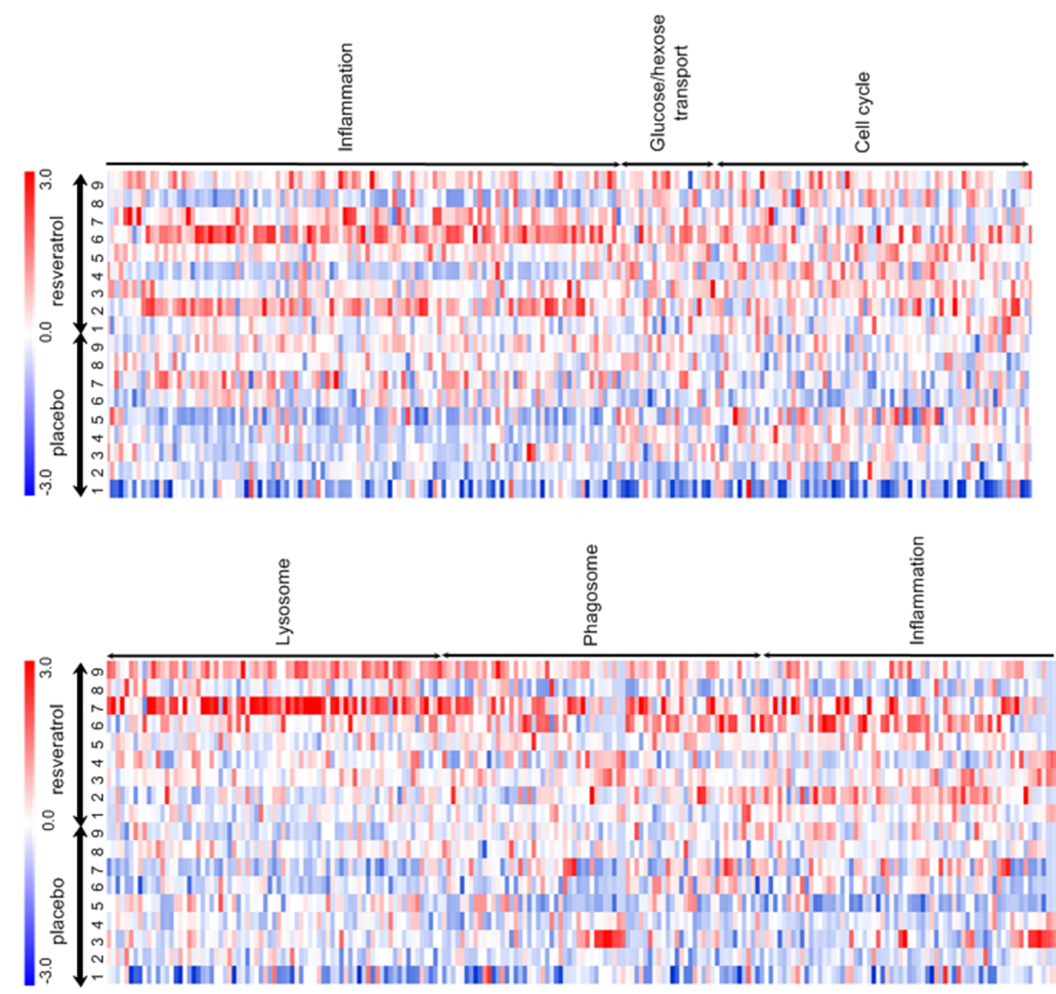

๓

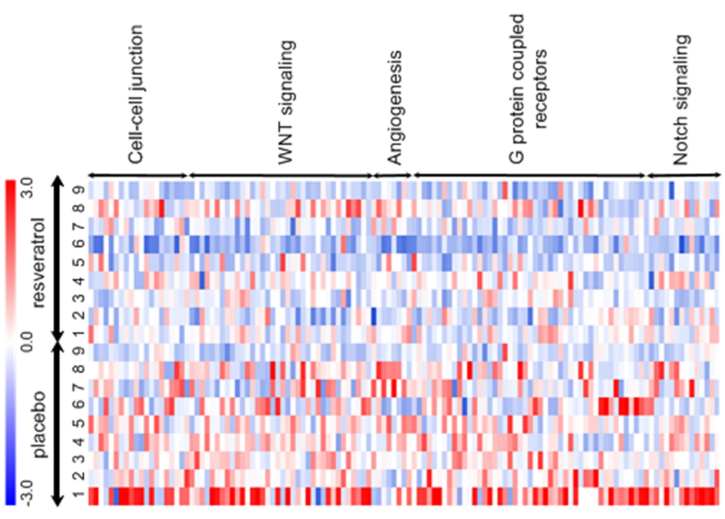

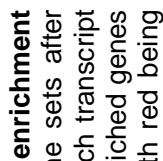

ब

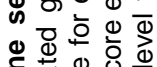

๘

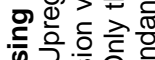

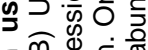

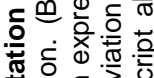

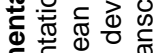
능 을

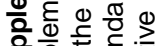

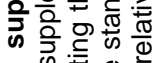
옿원

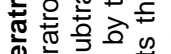
ब的苟 ఖ 인 융 む亦

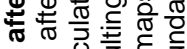

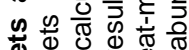
$\Phi$ 边 क)

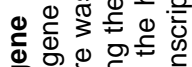
ब잉

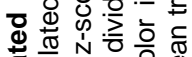
흑

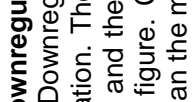
응 㐘

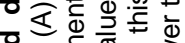
产宗要 능응웡 을엉 윽 ง 응 원

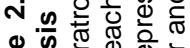
늑

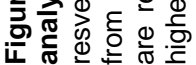




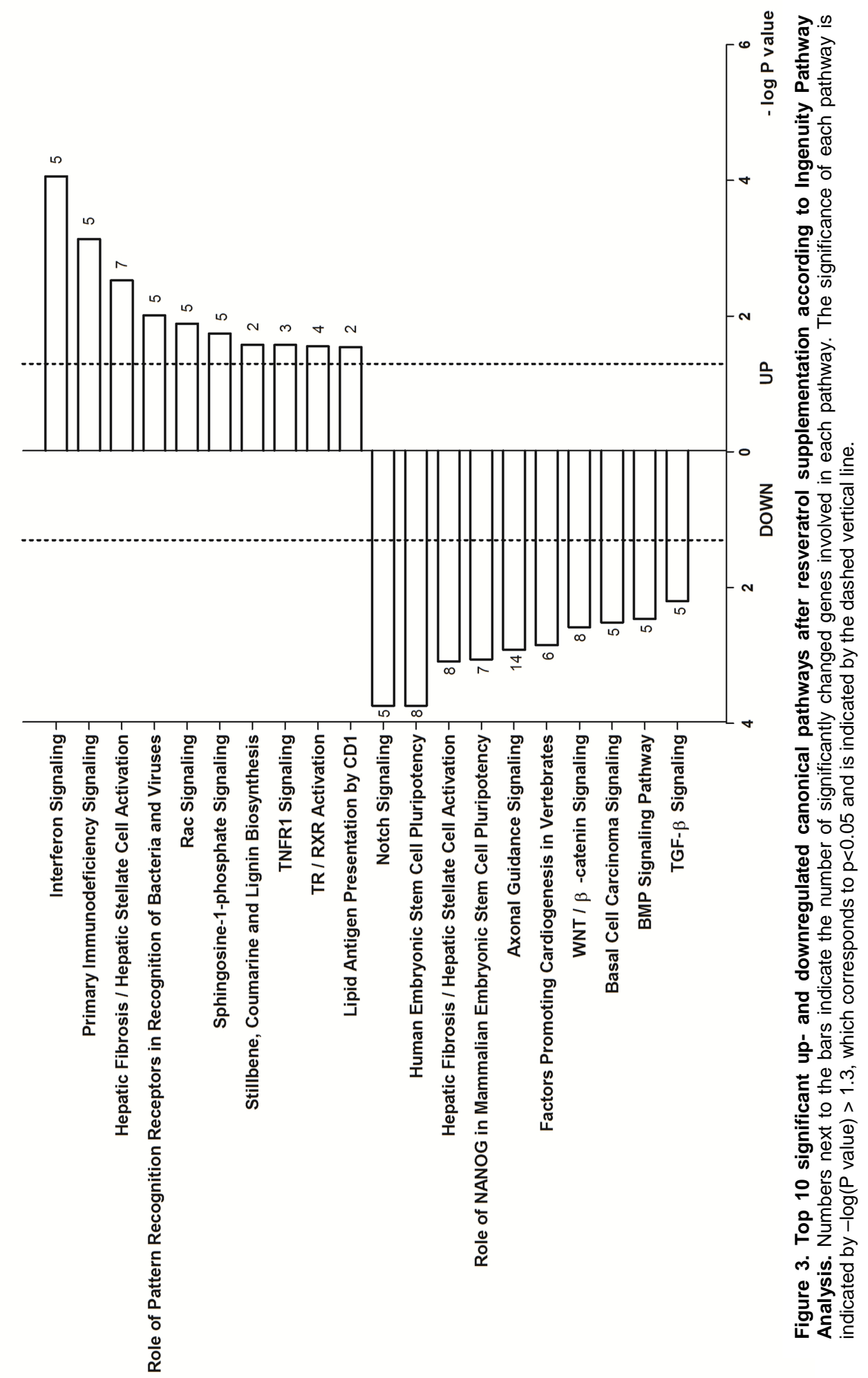




\section{Transcription factor analysis}

Several transcription factors involved in interferon signaling were identified as potentially activated (Table 1). Furthermore, the NFKB complex involved in inflammation, peroxisome proliferator-activated receptor $\delta$ (PPAR $\delta$ ) and transcription factor EB (TFEB), which links autophagy to lysosomal biogenesis, were shown to be activated (7).

Transcription factors that were identified as inhibited belonged to the Notch, BMP and TGF- $\beta$ signaling pathway including Notch 4 and mothers against decapentaplegic homolog 4 (SMAD4) (Table 1). The transcription factor hypoxia inducible factor $1 \alpha$ (HIF-1 $\alpha$ ) was also inhibited.

Table 1. Transcription factor analysis using Ingenuity.

\begin{tabular}{llccc}
\hline $\begin{array}{l}\text { Transcription } \\
\text { Regulator }\end{array}$ & Name & $\begin{array}{c}\text { Predicted } \\
\text { Activation } \\
\text { State }\end{array}$ & $\begin{array}{c}\text { Z- } \\
\text { score }\end{array}$ & P-value \\
\hline IRF7 & interferon regulatory factor 7 & Activated & 3.559 & $6.34 \mathrm{E}-05$ \\
STAT1 & signal transducer and activator of transcription 1 & Activated & 2.903 & $1.11 \mathrm{E}-02$ \\
IRF1 & interferon regulatory factor 1 & Activated & 2.844 & $1.76 \mathrm{E}-03$ \\
TFEB & transcription factor EB & Activated & 2.508 & $3.48 \mathrm{E}-05$ \\
NFkB complex & nuclear factor of kappa light polypeptide gene & Activated & 2.470 & $2.90 \mathrm{E}-03$ \\
SIM1 & enhancer in B-cells & Activated & 2.210 & $3.07 \mathrm{E}-01$ \\
PPARD & single-minded homolog 1 & Activated & 2.178 & $3.74 \mathrm{E}-01$ \\
IRF5 & peroxisome proliferator-activated receptor delta & Activated & 2.147 & $7.47 \mathrm{E}-03$ \\
ZNF217 & interferon regulatory factor 5 & Activated & 2.082 & $3.56 \mathrm{E}-02$ \\
CREB1 & zinc finger protein 217 & Inhibited & -2.034 & $3.80 \mathrm{E}-01$ \\
NOTCH4 & cAMP responsive element binding protein 1 & Inhibited & -2.254 & $2.10 \mathrm{E}-04$ \\
PGR & notch 4 & Inhibited & -2.272 & $5.22 \mathrm{E}-01$ \\
SMAD4 & progesterone receptor & Inhibited & -2.471 & $1.15 \mathrm{E}-01$ \\
HIF1A & SMAD family member 4 & Inhibited & -2.999 & $8.05 \mathrm{E}-03$ \\
TRIM24 & hypoxia inducible factor 1, alpha subunit & Inhibited & -3.165 & $6.91 \mathrm{E}-05$ \\
\hline
\end{tabular}

\section{Discussion}

This is the first human study describing the effects of 30 days resveratrol supplementation on adipose tissue morphology and gene expression patterns using microarray analysis. Resveratrol significantly decreased adipocyte size, with a shift towards a lower proportion of large and very large adipocytes and an increased proportion of small adipocytes. This reduced mean adipocyte size may underlie the previously reported improved insulin sensitivity, since adipocyte size is an independent determinant of insulin sensitivity in humans (8). Microarray analysis indicated an increase in adipogenesis and activation of the alternative pathway of 
lipid breakdown by lysosomes, which may explain the shift from large to small adipocytes.

The Wnt signaling, Notch signaling and BMP/TGF- $\beta$ signaling pathway were all downregulated after resveratrol supplementation. Inhibition of these signaling pathways has shown to result in adipogenesis of preadipocytes and/or multipotent precursor cells (9-12), although the exact role of these pathways in adipogenesis needs to be further elucidated. Induction of differentiation also stimulates mitotic clonal expansion. During this phase, growth-arrested preadipocytes reenter the cell cycle and undergo one or two rounds of cell division (13). Indeed, in our study, gene sets associated with cell cycle were upregulated by resveratrol. Overall, the downregulation in Wnt, Notch and BMP/TGF- $\beta$ signaling and upregulation of gene sets contributing to cell cycle after 30 days resveratrol supplementation suggest an enhanced adipogenesis in obese subjects. This increased adipogenesis could possibly explain the reduced adipocyte size after resveratrol. In contrast to our in vivo observations, in vitro experiments performed in murine and human adipocyte cell lines have shown that resveratrol inhibited proliferation and adipogenic differentiation $(14,15)$. The discrepancy between in vitro results and the present data may be explained by differences in resveratrol concentrations, acute in vitro vs. chronic in vivo administration and the presence of stromal vascular cell types (e.g. inflammatory cells) in the in vivo situation. Our data show that the angiogenesis pathway was downregulated in human adipose tissue after resveratrol supplementation. Furthermore, the transcription factor HIF-1 $\alpha$ whose activity is induced by hypoxia, insulin and nitric oxide was also downregulated (16). These data are consistent with studies showing that resveratrol possesses antiangiogenic effects (17), but further research is necessary to determine the exact role of resveratrol on angiogenesis in adipose tissue.

Besides a reduced expression of adipogenesis inhibiting pathways, activation of a lysosomal pathway of lipid breakdown was demonstrated. Until recently, mobilization of lipids by the classic lipolytic pathway has only been attributed to lipid droplet associated proteins and lipases. Lately, an alternative pathway of lipid metabolism has been proposed through the lysosomal degradative pathway of autophagy. Major support for the involvement of autophagy, is the activation of TFEB following resveratrol supplementation in the present study. TFEB controls multiple crucial steps of the autophagic pathway (7). Studies in hepatocytes and liver have demonstrated lipid breakdown by autophagy (18). Increased lysosomerelated proteolysis of the lipid droplet-associated protein perilipin (PLIN1) has been proposed, which could facilitate adipose tissue lipolysis (19). In preadipocytes, it has been shown that inhibition of autophagy may block adipogenesis, which was evident from a decrease in PPAR $\gamma$ and C/EBP $\alpha$ (20). Therefore, in the present study, the lysosome and phagosome pathways, may have contributed both to an 
induction of adipogenesis as well as to alternative lipid breakdown, possibly contributing to the shift toward an increased proportion of small adipocytes after resveratrol. Interestingly, autophagy may be induced upon starvation to deliver intracellular proteins and organelles to lysosomes for degradation and use as an energy source (21). Given the finding that resveratrol may partly mimic the effects of calorie restriction, it is plausible to suggest that resveratrol may induce autophagy, which has also been shown in cancer cells (22).

In the present study, several pathways involved in the immune response were upregulated after resveratrol treatment. This may reflect an increase in cellular stress induced by the reduction in adipocyte size caused by traction forces between the adipocyte and the surrounding extracellular matrix. This in turn may induce upregulation of inflammatory genes and pro-inflammatory adipokine production, as shown after weight loss $(23,24)$. On the other hand, upregulation of immune response pathways may be a physiological response to a higher lipid turnover (i.e. lysosomal lipid breakdown). We previously observed a decrease in plasma II- 6 and TNF- $\alpha$ concentrations and a downregulation of genes sets associated with immune response in skeletal muscle following resveratrol supplementation in humans (5). These findings indicate that the increased immune response in adipose tissue could represent a local rather than a systemic response (5).

Our previously reported results showed an enhanced insulin-mediated suppression of adipose tissue lipolysis and a slight improvement in insulin sensitivity represented by a reduced HOMA-IR, which may suggest an increased glucose uptake in adipose tissue (5). In line, the present results show that resveratrol increased pathways in the adipose tissue involved in glucose and hexose transport as evidenced by GSEA. However, in vitro data concerning the effects of resveratrol on glucose transport are conflicting. In mature human adipocytes, resveratrol stimulated basal and insulin stimulated glucose uptake (15). In contrast, in 3T3-L1 adipocytes resveratrol was found to decrease membrane-associated GLUT-4 protein levels and glucose uptake (25). It needs to be further established if the observed increase in glucose/hexose transport pathway, translates into a functional increase in glucose uptake.

In conclusion, resveratrol supplementation for 30 days induced a shift towards an increased proportion of metabolic favorable small adipocytes. This phenotype was accompanied by a gene expression profile indicative of increased adipogenesis and an alternative pathway of lipid breakdown. Interestingly the latter process seems to be mediated by pathways involving autophagy. These data suggest that adipose tissue is an important target tissue for the effects of resveratrol in humans, but further research is necessary to investigate whether it translates into an improved adipose tissue function. 


\section{Acknowledgments}

The authors thank Jos Stegen and Yvonne Essers for their excellent technical assistance with the biochemical analysis and adipocyte cell size analysis. The authors' contributions were as follows - E.B. and P.S. designed research; E.K. and S.T. conducted research; E.K. and M.B. analyzed data and performed statistical analysis; E.K. wrote the paper; S.T., M.B., G.G., J.J., L.A, E.M. and E.B contributed to discussion; S.T., M.B., G.G., J.J., L.A., M.M., P.S., E.M. and E.B. reviewed/edited the manuscript. E.B. is the guarantor of this work and, as such, had full access to all the data in the study and takes responsibility for the integrity of the data and the accuracy of the data analysis. This study was funded by Top Institute Food and Nutrition. TI Food and Nutrition, formerly known as WCFS, is a unique public/private partnership that generates vision on scientific breakthroughs in food and nutrition, resulting in the development of innovative products and technologies that respond to consumer demands for safe, tasty and healthy foods. Partners are major Dutch food companies and research organizations. The authors would like to thank DSM Nutritional Products Ltd., Kaiseraugst, Switzerland for providing us with the resVida ${ }^{\mathrm{TM}}$ and placebo capsules. 


\section{References}

1. Harris MI, Flegal KM, Cowie CC, et al. Prevalence of diabetes, impaired fasting glucose, and impaired glucose tolerance in U.S. adults. The Third National Health and Nutrition Examination Survey, 1988-1994. Diabetes Care 1998;21(4):518-24.

2. Szkudelska K, Szkudelski T. Resveratrol, obesity and diabetes. Eur J Pharmacol 2010;635(13):1-8.

3. Baur JA, Pearson KJ, Price NL, et al. Resveratrol improves health and survival of mice on a high-calorie diet. Nature 2006;444(7117):337-42.

4. Lagouge M, Argmann C, Gerhart-Hines Z, et al. Resveratrol improves mitochondrial function and protects against metabolic disease by activating SIRT1 and PGC-1alpha. Cell 2006;127(6):1109-22.

5. Timmers S, Konings E, Bilet L, et al. Calorie restriction-like effects of 30 days of resveratrol supplementation on energy metabolism and metabolic profile in obese humans. Cell Metab 2011;14(5):612-22.

6. Goossens $\mathrm{GH}$, Bizzarri A, Venteclef $\mathrm{N}$, et al. Increased adipose tissue oxygen tension in obese compared with lean men is accompanied by insulin resistance, impaired adipose tissue capillarization, and inflammation. Circulation 2011;124(1):67-76.

7. Settembre C, Di Malta C, Polito VA, et al. TFEB links autophagy to lysosomal biogenesis. Science 2011;332(6036):1429-33.

8. Goossens $\mathrm{GH}$. The role of adipose tissue dysfunction in the pathogenesis of obesity-related insulin resistance. Physiol Behav 2008;94(2):206-18.

9. Christodoulides C, Laudes M, Cawthorn WP, et al. The Wnt antagonist Dickkopf-1 and its receptors are coordinately regulated during early human adipogenesis. J Cell Sci 2006;119(Pt 12):2613-20.

10. Garces C, Ruiz-Hidalgo MJ, Font de Mora J, et al. Notch-1 controls the expression of fatty acid-activated transcription factors and is required for adipogenesis. $\mathrm{J}$ Biol Chem 1997;272(47):29729-34.

11. Ahrens M, Ankenbauer T, Schroder D, Hollnagel A, Mayer H, Gross G. Expression of human bone morphogenetic proteins- 2 or -4 in murine mesenchymal progenitor $\mathrm{C} 3 \mathrm{H} 10 \mathrm{~T} 1 / 2$ cells induces differentiation into distinct mesenchymal cell lineages. DNA Cell Biol 1993;12(10):871-80.

12. Tang $\mathrm{QQ}$, Otto TC, Lane MD. Commitment of $\mathrm{C} 3 \mathrm{H} 10 \mathrm{~T} 1 / 2$ pluripotent stem cells to the adipocyte lineage. Proc Natl Acad Sci U S A 2004;101(26):9607-11.

13. Patel YM, Lane MD. Mitotic clonal expansion during preadipocyte differentiation: calpainmediated turnover of p27. J Biol Chem 2000;275(23):17653-60.

14. Picard F, Kurtev M, Chung N, et al. Sirt1 promotes fat mobilization in white adipocytes by repressing PPAR-gamma. Nature 2004;429(6993):771-6.

15. Fischer-Posovszky $\mathrm{P}$, Kukulus V, Tews $\mathrm{D}$, et al. Resveratrol regulates human adipocyte number and function in a Sirt1-dependent manner. Am J Clin Nutr 2010;92(1):5-15.

16. He Q, Gao Z, Yin J, Zhang J, Yun Z, Ye J. Regulation of HIF-1\{alpha\} activity in adipose tissue by obesity-associated factors: adipogenesis, insulin, and hypoxia. Am J Physiol Endocrinol Metab 2011;300(5):E877-85.

17. Brakenhielm E, Cao R, Cao Y. Suppression of angiogenesis, tumor growth, and wound healing by resveratrol, a natural compound in red wine and grapes. Faseb J 2001;15(10):1798-800.

18. Singh R, Cuervo AM. Lipophagy: connecting autophagy and lipid metabolism. Int J Cell Biol 2012;2012:282041.

19. Kovsan J, Ben-Romano R, Souza SC, Greenberg AS, Rudich A. Regulation of adipocyte lipolysis by degradation of the perilipin protein: nelfinavir enhances lysosome-mediated perilipin proteolysis. J Biol Chem 2007;282(30):21704-11.

20. Singh $R$, Xiang $Y$, Wang $Y$, et al. Autophagy regulates adipose mass and differentiation in mice. J Clin Invest 2009;119(11):3329-39.

21. Finn PF, Dice JF. Proteolytic and lipolytic responses to starvation. Nutrition 2006;22(7-8):83044.

22. Opipari AW, Jr., Tan L, Boitano AE, Sorenson DR, Aurora A, Liu JR. Resveratrol-induced autophagocytosis in ovarian cancer cells. Cancer Res 2004;64(2):696-703.

23. Capel F, Klimcakova E, Viguerie N, et al. Macrophages and adipocytes in human obesity: adipose tissue gene expression and insulin sensitivity during calorie restriction and weight stabilization. Diabetes 2009;58(7):1558-67. 
24. Siklova-Vitkova M, Klimcakova E, Polak J, et al. Adipose Tissue Secretion and Expression of Adipocyte-Produced and Stromavascular Fraction-Produced Adipokines Vary during Multiple Phases of Weight-Reducing Dietary Intervention in Obese Women. J Clin Endocrinol Metab 2012;97(7):E1176-81.

25. Floyd ZE, Wang ZQ, Kilroy G, Cefalu WT. Modulation of peroxisome proliferator-activated receptor gamma stability and transcriptional activity in adipocytes by resveratrol. Metabolism 2008;57(7 Suppl 1):S32-8. 


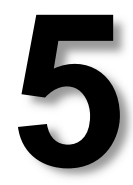

\title{
Polyunsaturated fatty acids
}

\section{acutely affect triacylglycerol-derived \\ skeletal muscle fatty acid uptake and}

increases postprandial

insulin sensitivity

\author{
Anneke Jans* \\ Ellen Konings* \\ Gijs H. Goossens \\ Freek G. Bouwman \\ Chantalle C. Moors \\ Mark V. Boekschoten \\ Lydia A. Afman \\ Michael Müller \\ Edwin C. Mariman \\ Ellen E. Blaak \\ * These authors contributed equally
}

Am J Clin Nutr. 2012 Apr;95(4):825-36. 


\section{Abstract}

Background: Dietary fat quality may influence skeletal muscle lipid handling and fat accumulation, thereby modulating insulin sensitivity.

Aim: The objective of the present study was to examine acute effects of meals with various fatty acid (FA) compositions on skeletal muscle $F A$ handling and postprandial insulin sensitivity in obese insulin resistant men.

Methods: In a single-blind randomized crossover study, 10 insulin resistant men consumed three high-fat mixed-meals $(2.6 \mathrm{MJ})$, which were high in saturated FA (SFA), monounsaturated FA (MUFA) or polyunsaturated FA (PUFA). Fasting and postprandial skeletal muscle FA handling were examined by measuring arteriovenous concentration differences across the forearm muscle. $\left[{ }^{2} \mathrm{H}_{2}\right]$-palmitate was infused intravenously to label endogenous triacylglycerol (TAG) and FFA in the circulation and $\left[\mathrm{U}-{ }^{13} \mathrm{C}\right]$-palmitate was added to the meal to label chylomicron-TAG. Skeletal muscle biopsies were taken to assess intramuscular lipid metabolism and gene expression.

Results: Insulin and glucose responses (AUC) after the SFA meal were significantly higher compared with the PUFA meal $(p=0.006$ and 0.033 , respectively). Uptake of TAG-derived FA was lower in the postprandial phase after the PUFA meal as compared with the other meals $\left(\mathrm{AUC}_{60-240}, \mathrm{p}=0.02\right)$. Fractional synthetic rate of the TAG, DAG and PL pool was higher after the MUFA meal as compared with the SFA meal. PUFA induced less transcriptional down-regulation of oxidative pathways compared with other meals.

Conclusion: PUFA reduced TAG-derived skeletal muscle FA uptake, which was accompanied by higher postprandial insulin sensitivity, a more transcriptional oxidative phenotype and altered intramyocellular lipid partitioning and may therefore be protective against the development of insulin resistance. 


\section{Introduction}

Increased fat storage in non-adipose tissues like skeletal muscle (ectopic fat accumulation) is a strong indicator of insulin resistance (1). Conditions characterized by insulin resistance are often accompanied by adipose tissue dysfunction and a reduced lipid buffering capacity, which may lead to an increased supply of triacylglycerol (TAG) and free fatty acids (FFA) to non-adipose tissues (lipid overflow hypothesis) $(1,2)$. Together with an impaired capacity of skeletal muscle to oxidize fat (3-6), lipid overflow may cause excess fat storage in skeletal muscle. Increased lipid accumulation in skeletal muscle, in particular the accumulation of lipid metabolites like diacylglycerol (DAG), long-chain fatty acylCoA and ceramides, may reduce insulin action by interference with insulin signaling (7-10). Dietary intervention, in particular a reduction of saturated fat (SFA), may improve insulin sensitivity (11-13), possibly through effects on lipid overflow and skeletal muscle FA handling. Polyunsaturated FA (PUFA) may reduce lipid overflow in the circulation by increasing lipid uptake and storage in adipose tissue by inducing adipocyte differentiation (14). Furthermore, it has been found in human muscle cell lines that SFA accumulate preferentially as DAG, thereby activating protein kinase $C$ and inhibiting insulin signaling, whilst unsaturated $F A$ are readily converted to TAG (15). Furthermore, oleic acid accumulates to a lesser extent as DAG or TAG than palmitic acid and is more directed towards oxidation (16). The underlying mechanisms for the differential effect of FA on metabolism may relate to intracellular pathways that are differently tuned by FA subtypes. It has been shown that PUFA may regulate gene transcription within minutes, acting as agonists of peroxisome proliferator-activated receptors (PPARs) and sterol regulatory element-binding protein 1c (SREBP1c) (17). However, human in vivo studies investigating the effects of meals with different FA composition on skeletal muscle FA handling are lacking. Recently, a unique dual stable isotope tracer approach was validated to study skeletal muscle FA metabolism in detail. This offers, in combination with measurements of arterio-venous concentration differences across the forearm muscle $(6,18)$, the possibility of differentiation between the metabolic fate of dietary versus endogenous FA. The hypothesis of the present study was that a meal high in unsaturated FA may acutely improve insulin sensitivity by reducing lipid overflow and shifting FA partitioning within muscle towards oxidation as compared with a meal high in SFA in obese insulin resistant subjects. The objective of the present study was to examine the acute effects of meals with various FA compositions on forearm muscle FA handling and postprandial insulin sensitivity in obese insulin resistant men using the dual stable isotope technique. Furthermore, baseline and postprandial skeletal muscle biopsies were collected for assessment of intramuscular FA handling and the transcriptional regulation of FA metabolism. 


\section{Methods}

10 insulin resistant men (age 50-70 years; BMI $29-39 \mathrm{~kg} / \mathrm{m}^{2} ; \mathrm{HOMA}_{\mathrm{R}}>2.5$ ) participated in a single-blind randomized cross-over study (Table 1). Exclusion criteria were weight change of $>3 \mathrm{~kg}$ within the last 3 months before the study; diabetes; chronic inflammatory conditions; kidney or liver dysfunction; use of hypolipidemic or anti-inflammatory medication; use of $\beta$-blockers; use of aspirin $>1$ per week; highly-trained athletes; and alcohol abuse. All subjects were informed about the nature of the study and written informed consent was obtained before study participation. The local Medical Ethical Committee of Maastricht University Medical Centre approved the study protocol.

Table 1. Subject characteristics.

\begin{tabular}{lc}
\hline & NGT $(\mathbf{n}=\mathbf{1 0})$ \\
\hline Age $(\mathrm{yrs})$ & $61.5 \pm 1.6$ \\
Body weight $(\mathrm{kg})$ & $98.1 \pm 2.8$ \\
BMI $\left(\mathrm{kg} / \mathrm{m}^{2}\right)$ & $33.8 \pm 3.8$ \\
Body fat $(\%)$ & $31.6 \pm 0.9$ \\
Fat free mass $(\mathrm{kg})$ & $70.3 \pm 1.8$ \\
Fat mass $(\mathrm{kg})$ & $28.1 \pm 1.6$ \\
Waist-hip ratio & $1.04 \pm 0.01$ \\
FPG (mmol/L) & $5.6 \pm 0.1$ \\
2h PG (mmol/L) & $6.2 \pm 0.4$ \\
Fasting insulin $(\mu \mathrm{U} / \mathrm{L})$ & $13.7 \pm 1.3$ \\
HOMA & $3.5 \pm 0.4$ \\
M-value $\left(\mu \mathrm{mol}{ }^{\star} \mathrm{kgBW}^{-1 *} \mathrm{~min}^{-1}\right)$ & $3.4 \pm 0.8$ \\
HbA1c $(\%)$ & $5.7 \pm 0.2$ \\
\hline
\end{tabular}

Values are mean \pm SEM. NGT, normal glucose tolerant; FPG, fasting plasma glucose; PG, plasma glucose

\section{Experimental design}

The design of the study was a randomized cross-over design with three different treatments. On four occasions, subjects arrived at the university after an overnight fast. On the first day, subjects completed anthropometric measurements, a fasting blood sample was collected, and a hyperinsulinaemic-euglycaemic clamp was performed to measure insulin sensitivity, using a modification of the method described by DeFronzo et al. (19). On the other three days (2-3 weeks between measurements, in randomized order), subjects were studied under baseline conditions and for 4 hours after the ingestion of a liquid high-fat mixed-meal, which was high either in SFA, MUFA or PUFA. A dual stable isotope technique was used, to study the contribution of dietary TAG (labeled with $\left[\mathrm{U}-{ }^{13} \mathrm{C}\right]$-palmitate), and endogenous TAG, i.e. VLDL, and circulating FFA (both labeled with $\left[{ }^{2} \mathrm{H}_{2}\right]$-palmitate) 
to lipid overflow and skeletal muscle FA handling, as reported previously $(6,18$, 20).

\section{Test meal composition}

The liquid test meal provided $2.6 \mathrm{MJ}$, consisting of $61 \mathrm{E} \%$ fat, $33 \mathrm{E} \%$ carbohydrates and $6.3 \mathrm{E} \%$ protein. Subjects were asked to drink the test meal within $5 \mathrm{~min}$. The FA composition of the test meals is presented in Table 2. The MUFA meal consisted out of $40 \mathrm{~g}$ olive oil and the PUFA meal contained $20 \mathrm{~g}$ safflower oil and $20 \mathrm{~g}$ fish oil (18\% EPA/12\% DHA) (Bioriginal, Den Bommel, The Netherlands).

Table 2. Fatty acid composition of test meals.

\begin{tabular}{|c|c|c|c|}
\hline & SFA & MUFA & PUFA \\
\hline Protein (E\%) & 6.3 & 6.5 & 6.5 \\
\hline $\mathrm{CH}(\mathrm{E} \%)$ & 32.6 & 32.3 & 32.3 \\
\hline Fat (E\%) & 61.2 & 61.2 & 61.2 \\
\hline SFA (E\%) & 35.5 & 11.6 & 14.4 \\
\hline MUFA (E\%) & 18.8 & 42.2 & 11.9 \\
\hline PUFA (E\%) & 1.7 & 4.6 & 34.8 \\
\hline
\end{tabular}

$\mathrm{CH}$, carbohydrate; SFA, saturated fatty acid; MUFA, monounsaturated; PUFA, polyunsaturated fatty acid; FFA, free fatty acids.

\section{Tracer infusion study}

Subjects arrived at the university after an overnight fast and were asked to refrain from drinking alcohol and to perform no strenuous exercise $24 \mathrm{~h}$ before the study day. In addition, they were asked to avoid food products naturally enriched with $\left[{ }^{13} \mathrm{C}\right]$ (i.e corn or pineapple) during the whole study period. Skeletal muscle metabolism was studied in the forearm muscle, using arterio-venous concentration differences corrected for blood flow. Three catheters were inserted before the start of the experiment. One catheter was placed retrogradely into a superficial dorsal hand vein, which was heated in a hot-box $\left(60^{\circ} \mathrm{C}\right)$ to obtain arterialized blood. In the same arm another catheter was placed in an antecubital vein for the infusion of the $\left[{ }^{2} \mathrm{H}_{2}\right]$-palmitate tracer. A third catheter was placed retrogradely in a deep antecubital vein of the contralateral forearm to sample venous blood draining the forearm muscle. After taking an arterialized and deep venous background sample at t-90 (i.e., $90 \mathrm{~min}$ before meal ingestion), a continuous intravenous infusion of the stable isotope tracer $\left[{ }^{2} \mathrm{H}_{2}\right]$-palmitate $(97 \%$ enrichment, Cambridge Isotope Laboratories, Andover, MA) complexed to albumin was started $\left(0.035 \mu \mathrm{mol}{ }^{*} \mathrm{~kg}\right.$ body weight $\left.{ }^{-1 *} \mathrm{~min}^{-1}\right)$. Baseline blood sampling was started after $1 \mathrm{~h}$ of tracer infusion to allow for isotopic equilibration to occur. Blood samples were taken simultaneously from the dorsal hand vein and the deep muscle vein at three time 
points during fasting (t-30, t-15, t0), and six time points postprandial ( $\mathrm{t} 30, \mathrm{t} 60, \mathrm{t} 90$, $\mathrm{t} 120, \mathrm{t} 180, \mathrm{t} 240$ ) after consumption of a high-fat mixed-meal (t0) containing $200 \mathrm{mg}$ $\left[\mathrm{U}-{ }^{13} \mathrm{C}\right]$-palmitate (98\% enrichment, Cambridge Isotope Laboratories). Forearm blood flow was measured before each blood sampling as described previously (21). Skeletal muscle biopsies were taken during fasting conditions (after placing the three catheters and before the background blood sampling) and at the end of the postprandial measurement period (t240).

\section{Indirect calorimetry}

Energy expenditure and whole-body substrate utilization were measured using an open-circuit ventilated hood system (Omnical, Maastricht University, The Netherlands) under fasting conditions and after meal ingestion (t30, t60, t90, t120, t180 and t240) (22). Gas analyses, which occurred every 15 seconds, were performed by dual paramagnetic $\mathrm{O}_{2}$ analyzers and dual infrared $\mathrm{CO}_{2}$ analyzers (type 1156, 1507, 1520; Servomex, Cowborough, Sussex, UK), similar to the analysis system described by Schoffelen et al. (23). Nitrogen excretion was calculated based on the assumption that protein oxidation represents $\sim 15 \%$ of total energy expenditure. Energy expenditure was calculated using the formula of Weir (24).

\section{Biochemical analyses}

Blood was collected in EDTA tubes, centrifuged (1000g, 10min, $4^{\circ} \mathrm{C}$ ) and plasma was immediately frozen in liquid nitrogen and stored at $-80^{\circ} \mathrm{C}$ until analysis. Plasma FFA was analyzed using standard enzymatic techniques automated on a Cobas Fara centrifugal spectrophotometer (Roche Diagnostics, Basel, Switzerland). Plasma TAG, glycerol, glucose, and lactate were analyzed enzymatically on a Cobas Mira automated spectrophotometer. Plasma insulin was measured with a double antibody radioimmunoassay (Linco Research, St Charles, $\mathrm{MO}$ ). Hematocrit was determined in heparinized blood using a microcapillary system. To determine isotope enrichment of plasma FFA and TAG, total lipids were extracted from the plasma using chloroform-methanol 2:1 (vol/vol). The FFA and TAG fractions were separated by thin-layer chromatography and derivatized to their methyl esters for the analysis of plasma palmitate. Plasma fractions were analyzed for the ${ }^{13} \mathrm{C}$-to- ${ }^{12} \mathrm{C}$ ratio on a gas chromatography continuous-flow isotope ratio-mass spectrometer (Finnigan MAT-252 GC-IRMS, Bremen, Germany) and for enrichment of $\left.{ }^{2} \mathrm{H}_{2}\right]$ (Finnigan Incos-XL GC-MS). The methyl ester of palmitate contains 17 carbon atoms and therefore, the tracer/tracee ratio (TTR) of palmitate was corrected for the extra methyl group. Plasma palmitate concentrations $(\mu \mathrm{mol} / \mathrm{L})$ were analyzed on an analytical GC with ion flame detection using heptadecanoic acid as internal standard. 


\section{Skeletal muscle biopsy}

Skeletal muscle biopsies were taken at baseline (after placing the three catheters and before the background blood sampling) and at the end of the postprandial measurement period (t240). Biopsies were obtained from the vastus lateralis muscle under local anesthesia of the skin and fascia using the Bergström method with suction (25), cleaned from any visible fat and blood, immediately frozen in isopentane at its melting point, and stored at $-80^{\circ} \mathrm{C}$ until analysis. The biopsies were taken to determine intramuscular TAG, DAG, FFA, and phospholipid (PL) content, their degree of saturation, as well as the fractional synthetic rate (FSR) of TAG, DAG, and PL (see skeletal muscle lipids). We investigated gene expression profiles using microarray.

\section{Skeletal muscle lipids}

Skeletal muscle biopsies were lyophilized and dissected free of extramyocellular lipid, blood, and connective tissue. Total lipids were extracted from 10 to $20 \mathrm{mg}$ muscle sample using chloroform-methanol (2:1 vol/vol) and internal standards, and thereafter evaporated under nitrogen at $37^{\circ} \mathrm{C}$. The extracted lipids were separated into TAG, DAG, FFA, and PL by thin-layer chromatography and transferred into tubes for methylation. The TAG and DAG fractions were methylated by adding 1 $\mathrm{mL}$ of toluene-methanol-(BF3-methanol $14 \%) \quad(20 \%-55 \%-25 \% \mathrm{vol} / \mathrm{vol})$ and incubated in capped tubes for $30 \mathrm{~min}$ at $100 \stackrel{\circ}{\circ}$. The $\mathrm{PL}$ fraction was methylated by adding $1 \mathrm{~mL}$ of (BF3-methanol 14\%) and incubation in capped tubes for $90 \mathrm{~min}$ at $100^{\circ} \mathrm{C}$. The FFA fraction was methylated by adding $1 \mathrm{~mL}$ of methanol-(BF3methanol $14 \%)(50 \%-50 \% \mathrm{vol} / \mathrm{vol})$ and incubation in capped tubes at room temperature for $15 \mathrm{~min}$. After incubation, $2 \mathrm{~mL}$ pentane was added to the samples, vortexed, and centrifuged $\left(1000 \mathrm{~g}, 5 \mathrm{~min}, 20^{\circ} \mathrm{C}\right.$ ), followed by isolation of pentane extracts (upper phase) and evaporation under nitrogen at $30^{\circ} \mathrm{C}$. Finally, the residues were dissolved in iso-octane and concentrations of FA in the fractions were determined using an analytical GC. Stable isotope enrichment of the lipid fractions was determined by measuring the ${ }^{13} \mathrm{C}$-to- ${ }^{12} \mathrm{C}$ ratio on a gas chromatography continuous-flow isotope ratio-mass spectrometer (Finnigan MAT252).

\section{Skeletal muscle gene expression}

\section{RNA extraction}

Total RNA was extracted from frozen skeletal muscle specimens using TRIzol reagent (Invitrogen, Breda, the Netherlands). RNA quantity was measured with the ND-100 spectrophotometer (Isogen Life Science, ljsselstein, the Netherlands), and RNA integrity was analysed on an Agilent 2100 BioAnalyzer (Agilent Technologies, 
Amsterdam, The Netherlands) using nanochips according to manufacturer's instructions.

\section{Microarray processing}

Total RNA (100 ng/sample) was labeled by using Whole Transcript Sense Target Assay and hybridized to human whole-genome Affymetrix Gene 1.0 ST arrays targeting 19,793 unique genes (Affymetrix, Santa Clara, CA). Sample labeling, hybridization to chips, and image scanning were performed according to the manufacturers' instructions.

\section{Microarray data analysis}

Quality control was performed and fulfilled the criteria for array hybridization suggested by the Tumor Analysis Best Practices Working Group (26). Microarrays were analyzed by using the reorganized oligonucleotide probes as described by Dai et al (27). All individual probes for a gene were combined, which allowed the possibility of detecting overall transcription activity on the basis of the latest genome and transcriptome information instead of on the basis of the Affymetrix probe set annotation. Expression values were calculated with the Robust Multichip Average (RMA) method and normalized by using quantile normalization $(28,29)$. Only probe sets with normalized signals $>20$ on $>4$ arrays were defined as expressed and selected for analysis. Individual genes were defined as changed when comparison of the normalized signal intensities showed a false discovery rate (FDR) (30) $Q$ value $<0.05$ in a 2- tailed paired t test with Bayesian correction (Limma) (31). Data were analyzed with the use of Ingenuity Pathway Analysis version 6.0 (Ingenuity Systems, Redwood City, CA). Our analysis identified canonical pathways that were most significant to the data set. The analysis was performed for up- and down-regulated gene sets together. Array data have been submitted to the Gene Expression Omnibus GSE31901.

\section{cDNA synthesis and quantitative real-time polymerase chain reaction}

Real-time quantitative Polymerase Chain Reaction (PCR) was used to analyze RNA samples. Total RNA was reverse-transcribed in a $20 \mu \mathrm{l}$ reaction using the iScript cDNA synthesis Kit (Bio-Rad,Veenendaal, the Netherlands) according to the manufacturer's instructions. Primers (available upon request) were designed using Beacon designer or were obtained by using Primer Bank. Real-time PCR was carried out in an icycler thermal cycler upgraded with a MyIQ ${ }^{\mathrm{TM}}$ Single Color RealTime PCR detection system (Bio-Rad, Veenendaal, the Netherlands). Gene expression levels were normalized using housekeeping genes $\beta$-actin and $\beta-2$ 
microglobulin ( $\beta 2 \mathrm{M})$. GeNorm was used to test the stability of the housekeeping genes (32).

\section{Calculations}

The homeostasis model assessment for insulin resistance $\left(\mathrm{HOMA}_{\mathrm{IR}}\right)$ was calculated according to Matthews et al. (33). The M-value from the hyperinsulinaemic-euglycaemic clamp was calculated as described by DeFronzo (19). Glucose and insulin AUC in response to the meal and $A \cup C_{\text {glucose }}{ }^{*} A U C_{\text {insulin }}{ }^{*} 10^{-6}$ (Plasma Glucose Index, PGI) were used as indices of postprandial insulin sensitivity $(34,35)$. Lower values of $P G I$ are indicative of better insulin sensitivity. The net flux of metabolites (labeled and unlabeled) across the forearm muscle was calculated by multiplying the arterio-venous concentration difference by forearm plasma flow. Plasma flow was calculated by multiplying forearm blood flow with (1-hematocrite)/100. A positive flux indicates net uptake across forearm muscle, whereas a negative flux indicates net release. Fractional extraction of metabolites (\%) was calculated as the arterio-venous concentration difference divided by the arterialized concentration. As a measure of efficiency of substrate removal from the circulation, clearance across forearm muscle was calculated by multiplying the fractional extraction with forearm plasma flow. Fasting rate of appearance of FFA (Ra $\left.a_{F A A}\right)\left(\mu \mathrm{mol}^{*} \mathrm{~kg}^{-1 *} \mathrm{~min}^{-1}\right)$ was calculated with Steele's equation for steady-state, whereas Steele's single-pool non-steady-state equations adapted for use with stable isotopes was used to calculate $\mathrm{RaFF}_{\mathrm{FF}}$ in the postprandial state (36). Labeled FFA and TAG concentrations were calculated as the product of TTR of $\left[{ }^{2} \mathrm{H}_{2}\right]$ - and $\left[\mathrm{U}-{ }^{13} \mathrm{C}\right]$-palmitate and the concentration of palmitate in FFA and TAG. Fractional extraction of $\left[\mathrm{U}-{ }^{13} \mathrm{C}\right]$-palmitate derived from the hydrolysis of $\left[\mathrm{U}-{ }^{13} \mathrm{C}\right]$-palmitate in TAG (i.e., percentage "entrapment") across forearm muscle was calculated as described previously, with the assumption that a proportion of FA derived from TAG hydrolysis may not be taken up by skeletal muscle $(6,18)$.

The FSR of skeletal muscle TAG, DAG, and PL were calculated using skeletal muscle FFA as the precursor pool for lipid synthesis (37). The increase in TTR of $\left[\mathrm{U}-{ }^{13} \mathrm{C}\right]$ from fasting to $4 \mathrm{~h}$ postprandial was divided by the enrichment of skeletal muscle FFA and expressed as percentage per hour $(\% / \mathrm{h})$. The degree of saturation of skeletal muscle TAG, DAG, PL, and FFA (\%) was calculated by dividing the sum of SFA by the total amount of FA in a fraction. 9-desaturase activity was estimated as the proportion palmitoleic acid (C16:1n-7) to palmitic acid (C16:0), and as the proportion oleic acid (C18:1n-9) to stearic acid (C18:0). Total skeletal muscle TAG, DAG, FFA, and PL contents were estimated as the sum of the particular FA content of the assessed fraction. Postprandial AUC of metabolites and substrate fluxes were calculated using the trapezoidal rule. Besides the total 
AUC (0-4 h after meal ingestion), also the "early" (0-2 h), and "mid" (2-4 h) AUC was calculated to obtain more detailed information about the time course of postprandial responses.

\section{Statistics}

Differences between the diets were examined using repeated-measures ANOVA (using AUC). When a significant diet by time interaction was observed, Bonferroni post-hoc testing was performed. Statistical analyses were performed using SPSS 19.0 for Mac (SPSS Inc., Chicago, IL, USA). All data are presented as mean \pm standard error of the mean (SEM). Statistical significance was set at $p<0.05$.

\section{Results}

\section{Arterialized metabolites, forearm blood flow and forearm muscle metabolism}

Fasting arterialized plasma glucose (Figure 1A) and insulin (Figure 1B) concentrations were comparable between the different meal test days. The postprandial arterialized plasma glucose and insulin concentrations were higher after the SFA meal then after the PUFA meal (Glucose: $p=0.006$ and insulin: $\mathrm{p}=0.033$, Table 3) with intermediate values after the MUFA meal. AUC for glucose and insulin, and the product of $A \cup C_{\text {glucose }}{ }^{*} A U C_{\text {insulin }}{ }^{*} 10^{-6}$ (PGI) were used as indices of insulin sensitivity. The PGI after the SFA meal $(12.8 \pm 1.5 \mathrm{PGl})$ was significantly higher as compared with the PUFA meal ( $8.5 \pm 0.8 \mathrm{PGI}, p=0.02)$. Net glucose flux (Figure 1C), arterialized lactate concentrations (Figure 1D) and net lactate flux (data not shown) were not different after intake of the different meals (Table 3). 
A

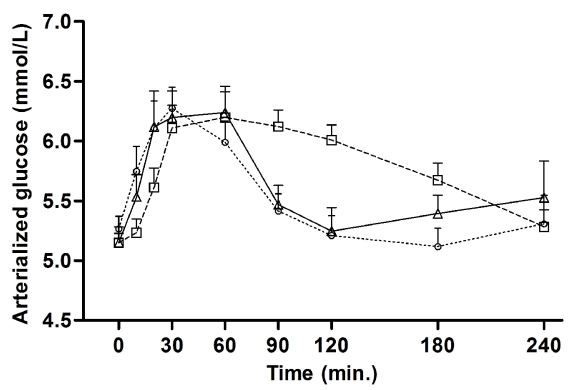

C

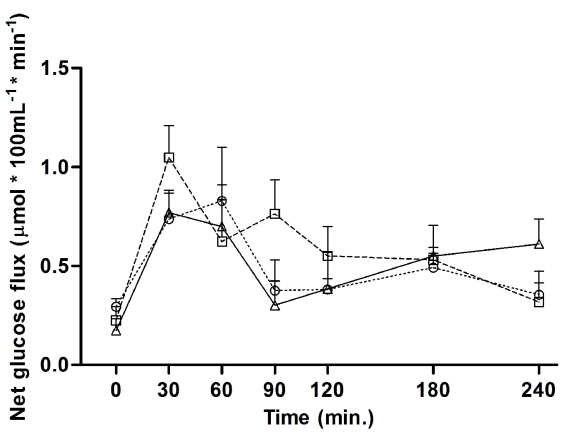

B $\quad-\because$ SFA

$\triangle$ MUFA

$\because$ PUFA

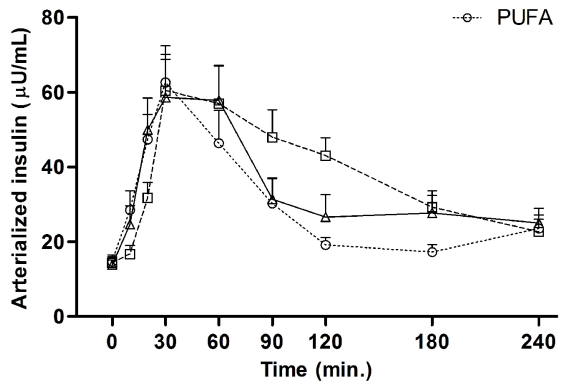

D

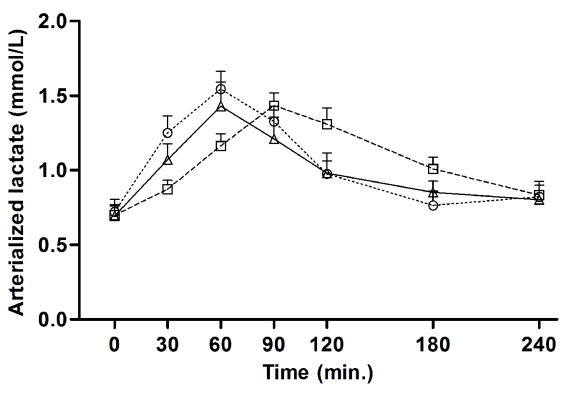

Figure 1. Postprandial arterialized glucose, insulin and lactate response. (A) Arterialized glucose, (B) arterialized insulin, (C) the net flux of glucose and (D) arterialized lactate concentrations under fasting conditions $(t=0)$ and after consumption of a high-fat mixed-meal. Values are mean $\pm S E M . n=10$. Repeated measures ANOVA were performed with Bonferroni post-hoc testing using the integrated responses (AUC) (Table 3).

\section{Whole-body and forearm muscle FFA metabolism}

\section{Whole-body FFA metabolism}

Postprandially, arterialized FFA concentrations decreased during the first $2 \mathrm{~h}$ and returned to fasting values after $4 \mathrm{~h}$ in all conditions (Figure 2A). FFA concentrations tended to be lower after the SFA meal in the mid phase (2-4h) compared with the PUFA meal ( $p=0.086$, Table 3$)$. $\left[{ }^{2} \mathrm{H}_{2}\right]$-labeled palmitate was infused intravenously and mixed with the plasma FFA pool. The tracer/tracee ratio (TTR) reached a steady state during fasting measurements (Figure 2B). Consistent with these findings, the $\mathrm{Ra}_{\mathrm{FFA}}$ in the circulation decreased after the meals, which is indicative of suppression of whole-body lipolysis (Figure $\mathbf{2 C}$ ). The $\mathrm{Ra}_{\mathrm{FFA}}$ was more reduced after the SFA meal in the postprandial phase as compared with the PUFA meal $\left(p=0.044\right.$, Table 3). $\left[U-{ }^{13} \mathrm{C}\right]$-labeled palmitate (resulting from spillover of FA derived from chylomicron-TAG hydrolysis) appeared in the plasma FFA pool from 60min after meal ingestion and concentrations increased throughout the postprandial period. There were no differences in arterialized concentrations of $\left[{ }^{2} \mathrm{H}_{2}\right]-$ or $\left[\mathrm{U}-{ }^{13} \mathrm{C}\right]-$ 
labeled palmitate in FFA after the three high-fat mixed-meals at any time (data not shown). The TTR of $\left[{ }^{2} \mathrm{H}_{2}\right]$-labeled palmitate in FFA was higher in arterialized versus deep venous plasma after all high-fat mixed-meals (SFA: $p=0.004$, MUFA: $p=0.002$, PUFA: $p=0.001$ ), indicating dilution of the $\left[{ }^{2} \mathrm{H}_{2}\right]$-tracer in the plasma FFA pool across forearm muscle (Figure 2B). The TTR of $\left[\mathrm{U}-{ }^{13} \mathrm{C}\right]$-palmitate in FFA was not different in arterialized versus deep venous plasma after the three high-fat mixed-meals (Figure 2D).

\begin{tabular}{|c|c|c|c|c|c|c|c|}
\hline & \multirow[b]{2}{*}{ SFA } & \multirow[b]{2}{*}{ MUFA } & \multirow[b]{2}{*}{ PUFA } & \multirow[b]{2}{*}{$\begin{array}{l}\text { ANOVA } \\
p \text {-value }\end{array}$} & \multicolumn{3}{|c|}{ Post-hoc } \\
\hline & & & & & $\begin{array}{c}\text { SFA } \\
\text { vs } \\
\text { MUFA }\end{array}$ & $\begin{array}{c}\text { SFA } \\
\text { vs } \\
\text { PUFA }\end{array}$ & $\begin{array}{c}\text { MUFA } \\
\text { vs } \\
\text { PUFA }\end{array}$ \\
\hline \multicolumn{8}{|c|}{ Arterialized Glucose (mmol/L per min) } \\
\hline Postprandial (0-4h) & $5.77 \pm 0.13$ & $5.59 \pm 0.14$ & $5.48 \pm 0.12$ & 0.003 & 0.139 & 0.006 & 0.301 \\
\hline Early & $5.94 \pm 0.15$ & $5.79 \pm 0.15$ & $5.74 \pm 0.13$ & 0.040 & 0.474 & 0.013 & 1.000 \\
\hline Mid & $5.61 \pm 0.14$ & $5.39 \pm 0.15$ & $5.22 \pm 0.15$ & 0.002 & 0.179 & 0.011 & 0.174 \\
\hline \multicolumn{8}{|c|}{ Arterialized Insulin ( $\mu \mathrm{U} / \mathrm{mL}$ per min) } \\
\hline Postprandial (0-4h) & $38.7 \pm 4.5$ & $34.5 \pm 4.1$ & $27.0 \pm 2.5$ & 0.028 & 0.681 & 0.033 & 0.387 \\
\hline Early & $46.3 \pm 6.2$ & $42.2 \pm 5.3$ & $34.7 \pm 4.3$ & 0.182 & NA & NA & NA \\
\hline Mid & $31.0 \pm 3.9$ & $26.8 \pm 3.6$ & $19.3 \pm 2.0$ & 0.006 & 0.243 & 0.021 & 0.305 \\
\hline
\end{tabular}

Net Glucose Flux across forearm muscle ( $\mu \mathrm{mol}^{\star} 100 \mathrm{~mL}^{-1 \star} \mathrm{min}^{-1}$ per $\mathrm{min}$ )

$\begin{array}{lllllllc}\text { Postprandial }(0-4 h) & 0.59 \pm 0.09 & 0.52 \pm 0.06 & 0.51 \pm 0.11 & 0.228 & \text { NA } & \text { NA } & \text { NA } \\ \text { Early } & 0.70 \pm 0.14 & 0.51 \pm 0.06 & 0.58 \pm 0.14 & 0.075 & 0.107 & 0.254 & 1.000 \\ \text { Mid } & 0.48 \pm 0.05 & 0.52 \pm 0.09 & 0.43 \pm 0.09 & 0.289 & \text { NA } & \text { NA } & \text { NA }\end{array}$

Arterialized Lactate (mmol/L per min)

$\begin{array}{lllllllc}\text { Postprandial (1-4h) } & 1.08 \pm 0.06 & 1.00 \pm 0.09 & 1.02 \pm 0.08 & 0.366 & \text { NA } & \text { NA } & \text { NA } \\ \text { Early } & 1.12 \pm 0.06 & 1.14 \pm 0.11 & 1.20 \pm 0.09 & 0.244 & \text { NA } & \text { NA } & \text { NA } \\ \text { Mid } & 1.04 \pm 0.08 & 0.87 \pm 0.07 & 0.83 \pm 0.09 & \mathbf{0 . 0 4 8} & 0.068 & 0.145 & 1.000\end{array}$

Arterialized FFA (mmol/L per min)

$\begin{array}{lllllllc}\text { Postprandial (0-4h) } & 336 \pm 17 & 377 \pm 33 & 377 \pm 26 & 0.193 & \text { NA } & \text { NA } & \text { NA } \\ \text { Early } & 374 \pm 28 & 360 \pm 33 & 350 \pm 29 & 0.704 & \text { NA } & \text { NA } & \text { NA } \\ \text { Mid } & 297 \pm 16 & 393 \pm 36 & 403 \pm 29 & \mathbf{0 . 0 1 9} & 0.142 & 0.086 & 1.000\end{array}$

Rate of appearance FFA $\left(\mu \mathrm{mol}^{*} \mathrm{~kg}^{-1 *} \mathrm{~min}^{-1}\right.$ per $\left.\mathrm{min}\right)$

$\begin{array}{lccccccc}\text { Postprandial (0-4h) } & 4.60 \pm 0.40 & 5.29 \pm 0.42 & 5.62 \pm 0.28 & \mathbf{0 . 0 1 9} & 0.202 & \mathbf{0 . 0 4 4} & 1.000 \\ \text { Early } & 5.09 \pm 0.43 & 5.71 \pm 0.47 & 5.86 \pm 0.36 & 0.174 & \text { NA } & \text { NA } & \text { NA } \\ \text { Mid } & 4.11 \pm 0.42 & 4.88 \pm 0.46 & 5.37 \pm 0.25 & \mathbf{0 . 0 2 1} & 0.357 & \mathbf{0 . 0 2 9} & 0.717\end{array}$


Arterialized TAG ( $\mu \mathrm{mol} / \mathrm{L}$ per $\mathrm{min})$

$\begin{array}{llllllll}\text { Postprandial (0-4h) } & 1716 \pm 192 & 1727 \pm 183 & 1424 \pm 180 & 0.377 & \text { NA } & \text { NA } & \text { NA } \\ \text { Early } & 1359 \pm 179 & 1274 \pm 162 & 1125 \pm 148 & 0.656 & \text { NA } & \text { NA } & \text { NA } \\ \text { Mid } & 2073 \pm 209 & 2181 \pm 208 & 1724 \pm 218 & 0.141 & \text { NA } & \text { NA } & \text { NA }\end{array}$

$\left[^{2} \mathrm{H}_{2}\right]$-labeled palmitate in TAG ( $\mu \mathrm{mol} / \mathrm{L}$ per min)

$\begin{array}{lccccccc}\text { Postprandial }(0-4 h) & 9.8 \pm 0.9 & 8.8 \pm 0.8 & 8.0 \pm 0.8 & \mathbf{0 . 0 3 6} & 0.208 & 0.176 & 0.496 \\ \text { Early } & 6.0 \pm 0.6 & 5.5 \pm 0.5 & 5.3 \pm 0.6 & 0.224 & \text { NA } & \text { NA } & \text { NA } \\ \text { Mid } & 13.6 \pm 1.3 & 12.0 \pm 1.2 & 10.6 \pm 1.1 & \mathbf{0 . 0 2 5} & 0.185 & 0.125 & 0.445\end{array}$

[U- ${ }^{13} \mathrm{C}$-labeled palmitate in TAG ( $\mu \mathrm{mol} / \mathrm{L}$ per $\left.\mathrm{min}\right)$

\begin{tabular}{lccccccc} 
Postprandial (0-4h) & $4.0 \pm 0.4$ & $7.0 \pm 1.0$ & $3.8 \pm 0.5$ & $\mathbf{0 . 0 2 4}$ & 0.149 & 1.000 & 0.079 \\
Early (1-2h) & $13.3 \pm 3.2$ & $22.2 \pm 10.2$ & $7.5 \pm 2.5$ & 0.249 & NA & NA & NA \\
Mid & $5.6 \pm 0.8$ & $9.9 \pm 1.7$ & $5.4 \pm 1.0$ & $\mathbf{0 . 0 1 9}$ & 0.107 & 1.000 & 0.069 \\
\hline
\end{tabular}

Postprandial data are expressed as AUC/min and based on postprandial period ( $0-4 \mathrm{~h}$ after meal ingestion) unless otherwise stated. Early is $0-2 \mathrm{~h}$ after meal ingestion and mid is $2-4 \mathrm{~h}$ after meal ingestion. SFA, saturated fatty acid; MUFA, monounsaturated; PUFA, polyunsaturated fatty acid; FFA, free fatty acids; TAG, triacylglycerol; NA, not applicable. Values are mean \pm SEM. $n=10$. $p$-value represents overall $p$-value of the repeated measures ANOVA. Post-hoc testing was performed using the Bonferroni correction.

\section{Forearm muscle FFA metabolism}

The net flux of plasma FFA across forearm muscle (arterio-venous concentration difference multiplied by forearm plasma flow) was not different after the high-fat mixed-meals (Table 4). At the same time, a consistent uptake of $\left[{ }^{2} \mathrm{H}_{2}\right]$-labeled palmitate FFA was observed after all high-fat mixed-meals (Table 4), with no differences between meals. 
A

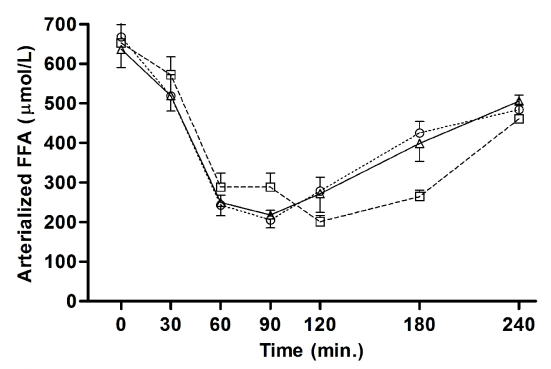

C

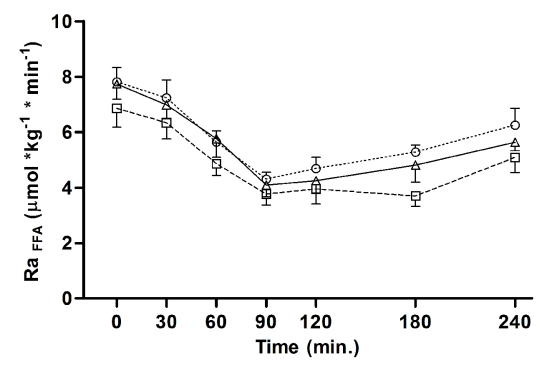

B $\quad \rightarrow$ SFA

$\triangle$ MUFA

.... PUFA

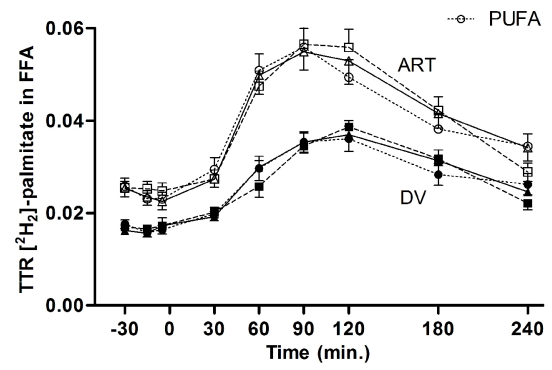

D

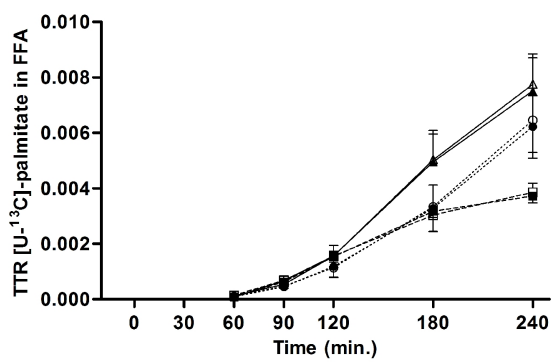

Figure 2. Postprandial whole body free fatty acid (FFA) metabolism. (A) Arterialized plasma free fatty acid (FFA) concentrations, (B) the tracer-tracee ratio (TTR) of $\left[{ }^{2} \mathrm{H}_{2}\right]$-palmitate, (C) the rate of appearance of FFA (Ra FFA) and (D) TTR of [U- $\left.{ }^{13} \mathrm{C}\right]$-palmitate. Values are mean \pm SEM. $n=10$. Repeated measures ANOVA were performed with Bonferroni post-hoc testing using the integrated responses (AUC) (Table 3). For figure $B$ and $D$ also paired student's t-test were performed to analyze the difference in TTR between arterialized and deep venous plasma. (B) The TTR of $\left[{ }^{2} \mathrm{H}_{2}\right]$-labeled palmitate in FFA was higher in arterialized versus deep venous plasma after all high-fat mixed-meals (iAUC: SFA: $p=0.004$, MUFA: $p=0.002$, PUFA: $p=0.001$ ). (D) The TTR of $\left.U-{ }^{13} \mathrm{C}\right]$-labeled palmitate was not different in arterialized versus deep venous plasma after the three high-fat mixed meals. (B and $D)$ There were no differences between the three high fat mixed meals. White symbols: arterialized plasma concentration (ART); Black symbols: deep venous plasma concentration (DV).

\section{Whole-body and forearm muscle TAG metabolism}

\section{Whole-body TAG metabolism}

Arterialized plasma TAG concentrations were similar between groups under fasting conditions and after ingestion of a high-fat mixed-meal (Figure $\mathbf{3 A}$ and Table $\mathbf{3}$ ). The $\left[{ }^{2} \mathrm{H}_{2}\right]$-palmitate tracer could be measured in plasma TAG from the first baseline sample onward, reflecting incorporation of the intravenously infused tracer into VLDL-TAG (Figure 3B). The $\left[\mathrm{U}-{ }^{13} \mathrm{C}\right]$-palmitate tracer, given with the meal, appeared in plasma TAG from 60min after meal ingestion, representing chylomicron-TAG in the circulation (Figure 3B). Both labeled TAG fractions increased up to $4 \mathrm{~h}$ postprandial, whilst the $\left[\mathrm{U}-{ }^{13} \mathrm{C}\right]$-labeled palmitate in TAG tended to increase more pronounced after the MUFA meal as compared with the PUFA meal $\left(p=0.079\right.$, Figure 3B and Table 3). The increase in $\left[{ }^{2} \mathrm{H}_{2}\right]$-labeled palmitate in TAG was most pronounced after the SFA meal ( $p=0.036$, Figure 3B and Table 3 ). 
A

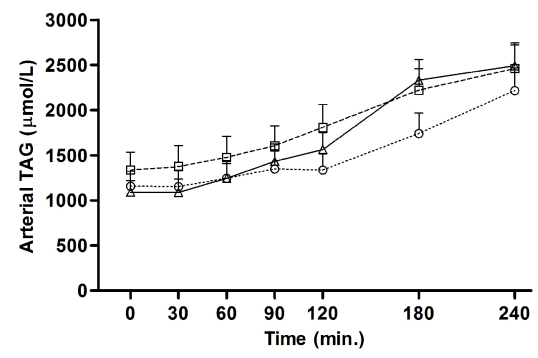

C

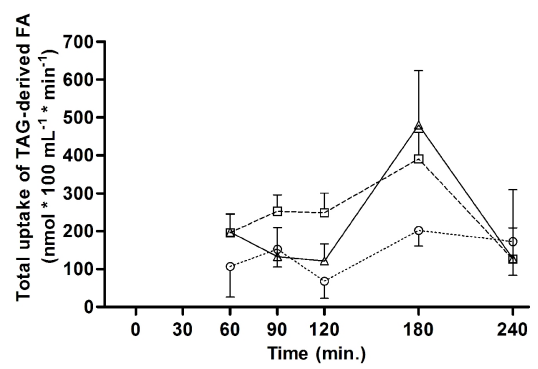

B

$-\because-\cdot$ SFA

$\triangle$ MUFA

-... PUFA

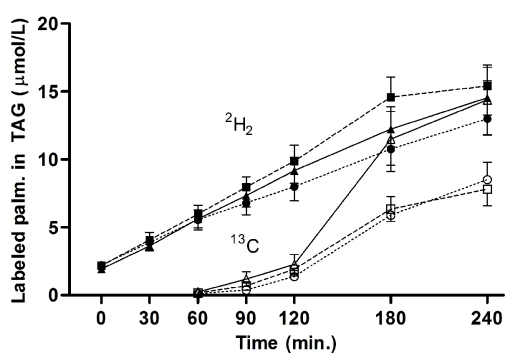

Figure 3. Postprandial whole triacylglycerol (TAG) metabolism. (A) Arterialized plasma triacylglycerol (TAG) concentrations, (B) $\left[{ }^{2} \mathrm{H}_{2}\right]-$ palmitate in TAG and $\left[\mathrm{U}{ }^{13} \mathrm{C}\right]$-palmitate in TAG, and $(C)$ total uptake of TAG-derived FA during fasting $(\mathrm{t}=0)$ and after consumption of a high-fat mixed-meal. Values are mean \pm SEM. $n=10$. Repeated measures ANOVA were performed with Bonferroni post-hoc testing using the integrated responses (AUC) (Table 3 and 4). Black symbols are $\left[{ }^{2} \mathrm{H}_{2}\right]$-palmitate and white symbols are $\left[{ }^{13} \mathrm{C}\right]$-palmitate in panel $\mathrm{C}$.

\section{Forearm muscle TAG metabolism}

Fractional extraction of $\left[\mathrm{U}-{ }^{13} \mathrm{C}\right]$-labeled palmitate in TAG across forearm muscle was consistently greater than the fractional extraction of $\left[{ }^{2} \mathrm{H}_{2}\right]$-labeled palmitate in TAG throughout the whole postprandial period (data not shown). There were no significant differences in extraction of $\left[{ }^{2} \mathrm{H}_{2}\right]$-labeled palmitate in TAG after the three high-fat mixed-meals (Table 4). Extraction of $\left[\mathrm{U}-{ }^{13} \mathrm{C}\right]$-labeled palmitate in TAG tended to be reduced after the PUFA meal compared with the MUFA meal in the early phase ( $p=0.06$, Table 4). The (unidirectional) uptake of FA derived from TAG hydrolysis across forearm muscle was reduced after the PUFA meal as compared with the SFA meal ( $p=0.02$, Figure $3 C$ and Table 4$)$.

Table 4. Fasting and postprandial FFA and TAG metabolism and forearm blood flow

\begin{tabular}{|c|c|c|c|c|}
\hline & SFA & MUFA & PUFA & P-value \\
\hline \multicolumn{5}{|c|}{ Forearm blood flow $\left(\mathrm{ml}^{\star} 100 \mathrm{~mL}^{-1 *} \mathrm{~min}^{-1}\right)$} \\
\hline Fasting & $3.28 \pm 0.53$ & $2.74 \pm 0.29$ & $2.65 \pm 0.21$ & 0.189 \\
\hline Postprandial (0-4h) & $3.25 \pm 0.41$ & $2.95 \pm 0.26$ & $2.59 \pm 0.26$ & 0.859 \\
\hline Early & $3.28 \pm 0.48$ & $2.90 \pm 0.27$ & $2.77 \pm 0.26$ & 0.725 \\
\hline Mid & $3.10 \pm 0.26$ & $2.87 \pm 0.23$ & $3.01 \pm 0.23$ & 0.628 \\
\hline
\end{tabular}


Net FFA flux across forearm muscle $\left(\mathrm{nmol}^{\star}{ }^{*} 100 \mathrm{~mL}^{-1 *} \mathrm{~min}^{-1}\right)$

$\begin{array}{lcccc}\text { Fasting } & 14.3 \pm 80.9 & 20.4 \pm 61.2 & 11.5 \pm 60.1 & 0.937 \\ \text { Postprandial (0-4h) } & 26.7 \pm 37.2 & 47.2 \pm 29.6 & 20.0 \pm 32.7 & 0.375 \\ \text { Early } & -14.0 \pm 52.2 & 6.7 \pm 39.1 & -20.2 \pm 35.0 & 0.195 \\ \text { Mid } & 67.3 \pm 24.0 & 87.6 \pm 22.0 & 64.5 \pm 37.5 & 0.601\end{array}$

$\left[^{2} \mathrm{H}_{2}\right]$-palmitate FFA net flux across forearm muscle $\left(\mathrm{nmol}^{*} 100 \mathrm{~mL}^{-1 *} \mathrm{~min}^{-1}\right)$

$\begin{array}{lllll}\text { Fasting } & 2.19 \pm 0.47 & 1.78 \pm 0.40 & 1.80 \pm 0.34 & 0.504 \\ \text { Postprandial (0-4h) } & 1.80 \pm 0.37 & 1.58 \pm 0.23 & 1.68 \pm 0.29 & 0.703 \\ \text { Early } & 1.78 \pm 0.40 & 1.49 \pm 0.32 & 1.48 \pm 0.32 & 0.271 \\ \text { Mid } & 1.83 \pm 0.38 & 1.66 \pm 0.32 & 1.89 \pm 0.33 & 0.826\end{array}$

$\left[^{13} \mathrm{C}\right]$-palmitate FFA net flux across forearm muscle $\left(\mathrm{nmol}^{\star} 100 \mathrm{~mL}^{-1 *} \min ^{-1}\right)$

$\begin{array}{lcccc}\text { Fasting } & \text { NA } & \text { NA } & \text { NA } & \\ \text { Postprandial (1-4h) } & 7.53 \pm 3.64 & 9.87 \pm 3.97 & 3.69 \pm 4.56 & 0.448 \\ \text { Early } & 0.07 \pm 0.10 & -0.07 \pm 0.12 & 0.02 \pm 0.03 & 0.505 \\ \text { Mid } & 0.06 \pm 0.03 & 0.08 \pm 0.03 & 0.03 \pm 0.04 & 0.408\end{array}$

Net TAG flux across forearm muscle $\left(\mathrm{nmol}^{\star} 100 \mathrm{~mL}^{-1 *} \mathrm{~min}^{-1}\right)$

$\begin{array}{lcccc}\text { Fasting } & 58.0 \pm 35.5 & 21.6 \pm 9.7 & 44.8 \pm 21.5 & 0.365 \\ \text { Postprandial (0-4h) } & 81.4 \pm 9.9 & 70.3 \pm 55.0 & 48.7 \pm 16.3 & 0.526 \\ \text { Early } & 85.6 \pm 33.8 & 47.6 \pm 10.8 & 44.6 \pm 16.3 & 0.904 \\ \text { Mid } & 74.4 \pm 25.2 & 87.2 \pm 24.1 & 68.9 \pm 15.3 & 0.647\end{array}$

$\left[^{2} \mathrm{H}_{2}\right]$-palmitate TAG net flux across forearm muscle $\left(\mathrm{nmol}^{*} 100 \mathrm{~mL}^{-1 *} \mathrm{~min}^{-1}\right)$

$\begin{array}{lcccc}\text { Fasting } & 0.05 \pm 0.27 & 0.12 \pm 0.21 & 0.38 \pm 0.14 & 0.479 \\ \text { Postprandial (0-4h) } & -0.08 \pm 0.30 & -0.35 \pm 0.20 & 0.18 \pm 0.18 & 0.277 \\ \text { Early } & 0.13 \pm 0.24 & 0.31 \pm 0.19 & 0.34 \pm 0.23 & 0.678 \\ \text { Mid } & -0.28 \pm 0.53 & -1.02 \pm 0.31 & 0.02 \pm 0.36 & 0.255\end{array}$

$I^{13} \mathrm{Cl}$-palmitate TAG net flux across forearm muscle $\left(\mathrm{nmol}^{*} 100 \mathrm{~mL}^{-1 *} \mathrm{~min}^{-1}\right)$

$\begin{array}{lcccc}\text { Fasting } & \text { NA } & \text { NA } & \text { NA } & \text { NA } \\ \text { Postprandial (0-4h) } & 0.35 \pm 0.18 & -0.18 \pm 0.18 & 0.12 \pm 0.30 & 0.256 \\ \text { Early } & 0.19 \pm 0.07 & 0.30 \pm 0.10 & 0.04 \pm 0.02^{\#} & 0.103 \\ \text { Mid } & 0.43 \pm 0.26 & -0.43 \pm 0.31 & 0.16 \pm 0.45 & 0.214\end{array}$

\section{Unidirectional uptake of fatty acids derived from TAG hydrolysis $\left(\mathrm{nmol}^{*} 100 \mathrm{~mL}^{-1 *} \mathrm{~min}^{-1}\right)$}

$\begin{array}{lllll}\text { Postprandial (1-4h) } & 271.5 \pm 41.9 & 250.3 \pm 61.4 & 147.3 \pm 41.8^{*} & 0.082 \\ \text { Early } & 237.3 \pm 28.2 & 146.5 \pm 46.9 & 119.8 \pm 40.4^{*} & 0.069 \\ \text { Mid } & 288.6 \pm 51.5 & 302.3 \pm 76.0 & 161.1 \pm 46.1^{*} & 0.094\end{array}$

Postprandial data are expressed as AUC/min and based on postprandial period ( $0-4 \mathrm{~h}$ after meal ingestion) unless otherwise stated. Early is $0-2 \mathrm{~h}$ after meal ingestion and mid is $2-4 \mathrm{~h}$ after meal ingestion. SFA, saturated fatty acid; MUFA, monounsaturated; PUFA, polyunsaturated fatty acid; FFA, free fatty acids; TAG, triacylglycerol; NA, not applicable. Values are mean \pm SEM. $n=10$. $p$-value represents overall $p$-value of the repeated measures ANOVA. Post-hoc testing was performed using the bonferroni correction. * SFA versus PUFA $p<0.05$. \# MUFA versus PUFA $\mathrm{p}<0.1$. 


\section{Energy expenditure and whole-body substrate metabolism}

Whole-body energy expenditure, respiratory quotient, carbohydrate oxidation and fat oxidation during fasting and after meal ingestion were comparable between the different meals (data not shown).

\section{Intramuscular lipid metabolism}

\section{Muscle lipid content and composition}

The content of skeletal muscle TAG, DAG, FFA, and PL did not change after the three high-fat mixed-meals (data not shown). The saturation of muscle TAG, DAG, $P L$ and FFA was not different after the ingestion of the different meals (data not shown). Also the individual FA in the different muscle lipid pools did not change after the three meals (data not shown).

Incorporation of $\left[\mathrm{U}^{13} \mathrm{C}\right]$-labeled palmitate in muscle lipids (FSR).

The fractional synthetic rate (FSR) of skeletal muscle DAG $(p=0.015)$ was higher after the MUFA meal and tended to be higher in the TAG $(p=0.067)$ and PL fraction $(p=0.08)$ as compared with the SFA meal (Figure 4). After the PUFA meal, FSR values were of similar magnitude as after the MUFA meal but were not statistically different as compared with the SFA meal. This may indicate that a higher proportion of palmitate from the intramuscular FFA pool was directed toward the muscle lipid pools after the unsaturated FA meals.

A

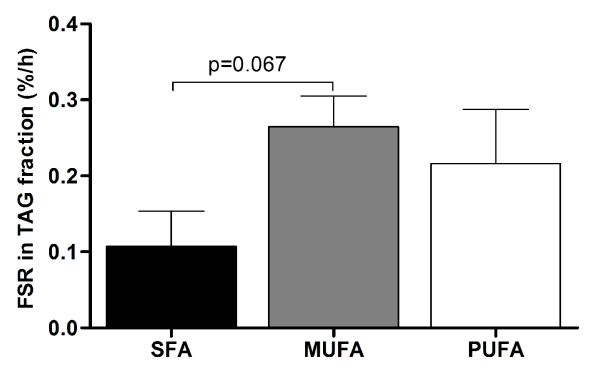

C

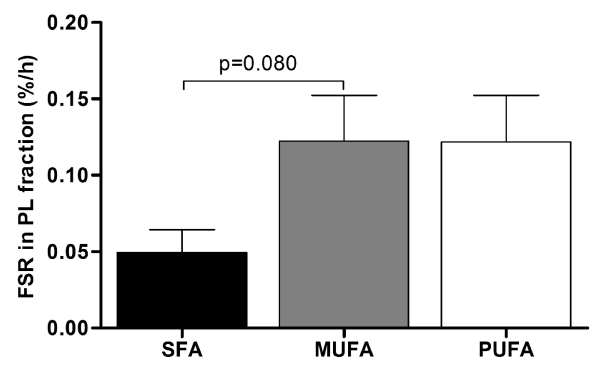

B

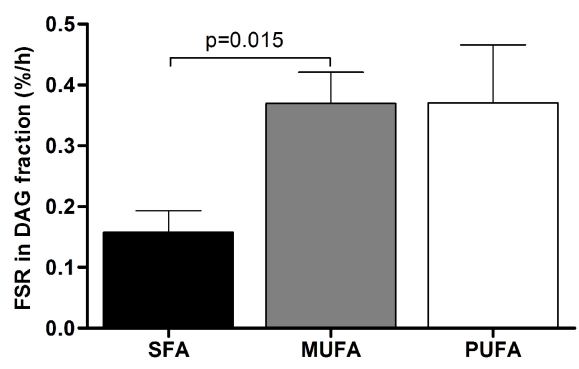

Figure 4. Fractional synthetic rate after the high-fat mixed-meals. The FSR in $(A)$ TAG, (B) DAG and (C) PL after consumption of a high-fat mixed-meal. DAG, Diacylglycerol; FSR, fractional synthetic rate; TAG, triacylglycerol. Values are mean \pm SEM. $n=8$. Paired student's $t$ tests were performed. 


\section{Chapter 5}

\section{Microarray analysis}

Microarray analysis was performed on skeletal muscle tissue before and 4 hours after consumption of a high-fat mixed-meal consisting of SFA, MUFA or PUFA. During 2 skeletal muscle biopsy procedures, we could not obtain enough biopsy material for further analysis From the 19,793 genes present on the array, 14,424 genes were defined as expressed in skeletal muscle (Figure 5A). Genes that showed comparable regulation after the three meals (5,429 genes) were not taken into account for further analysis (Figure 5B). About $66 \%$ of the genes that were changed after the SFA and MUFA meal were down-regulated, whereas $60 \%$ of the genes were down-regulated after the PUFA meal.

A

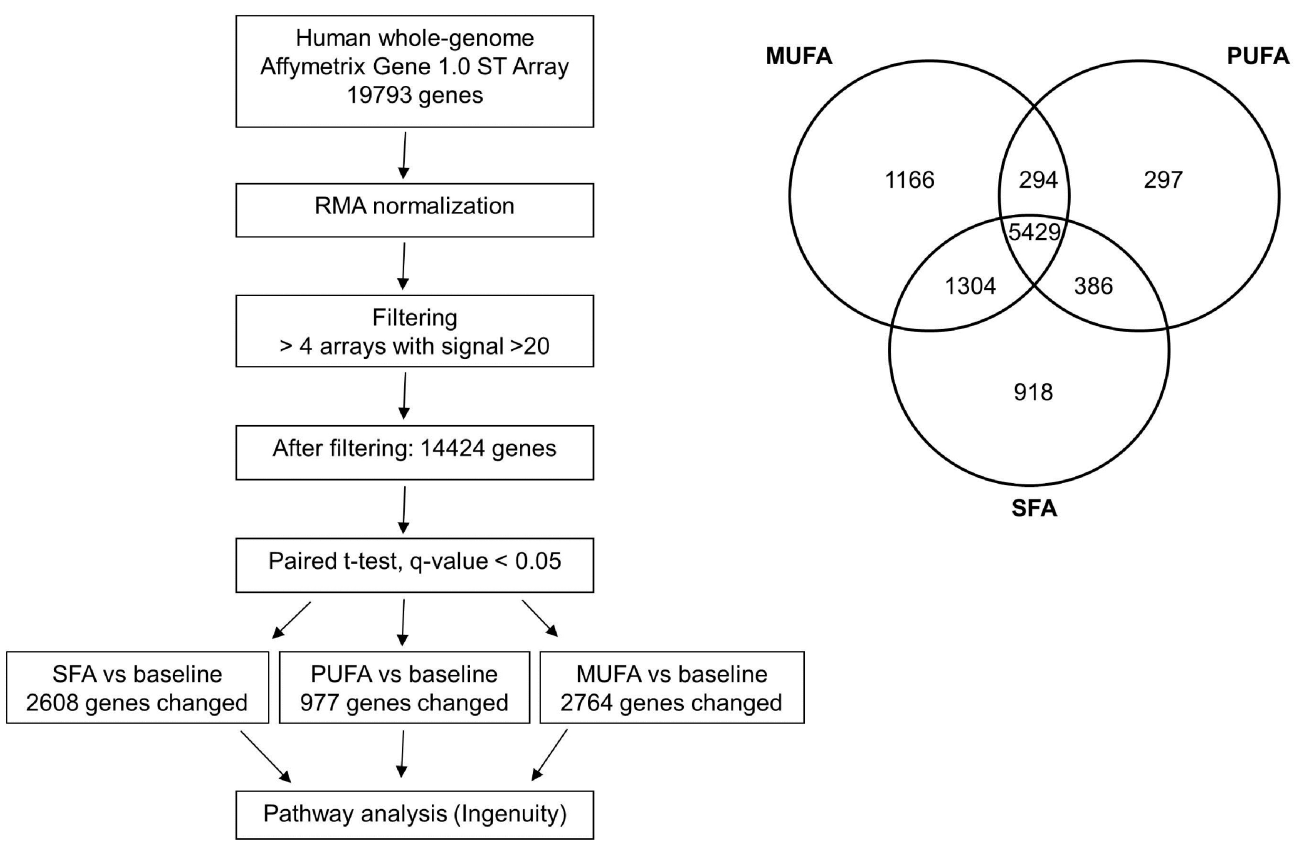

B

Figure 5. Gene selection procedures and criteria. (A) Flow chart of gene selection and number of genes changed in the microarray analysis. (B) Venn diagram of selected genes with a q-value $<0.05$. Overlapping genes were regulated in the same manner after the three meals and were not taken into account for further analysis. MUFA, monounsaturated fatty acid; PUFA, polyunsaturated fatty acid; RMA, robust multichip average; SFA, saturated fatty acid. 
To obtain further insight into the physiological relevance of these genes, pathway analysis was performed. The top 10 most significantly regulated pathways after each meal are listed in Figure 6. The pathways that were affected the most after consumption of the SFA and MUFA meal were those involved in ubiquinone biosynthesis, mitochondrial dysfunction and oxidative phosphorylation, which consisted of genes involved in complex I-V of the respiratory chain. Consumption of the SFA meal resulted in significantly decreased expression of 33 out of 96 OXPHOS genes, while 28 genes were down-regulated after the MUFA meal and 7 after the PUFA meal. Thus, there were less genes significantly down-regulated after the PUFA meal, indicating less down-regulation of mitochondrial function (data not shown). To confirm gene expression changes revealed by microarray analysis, GPCR was used. We selected genes involved in complex I-V of the respiratory chain. All genes were significantly down-regulated after the SFA meal, which confirmed the microarray analysis. NDUFA1, NDUFB3, COX17 and ATP5J were less down-regulated after the PUFA meal, so the change in expression of genes showed the same direction of change as the microarray, but the relatively small differences in expression of individual genes did not reach statistical significance between meals (data not shown). 


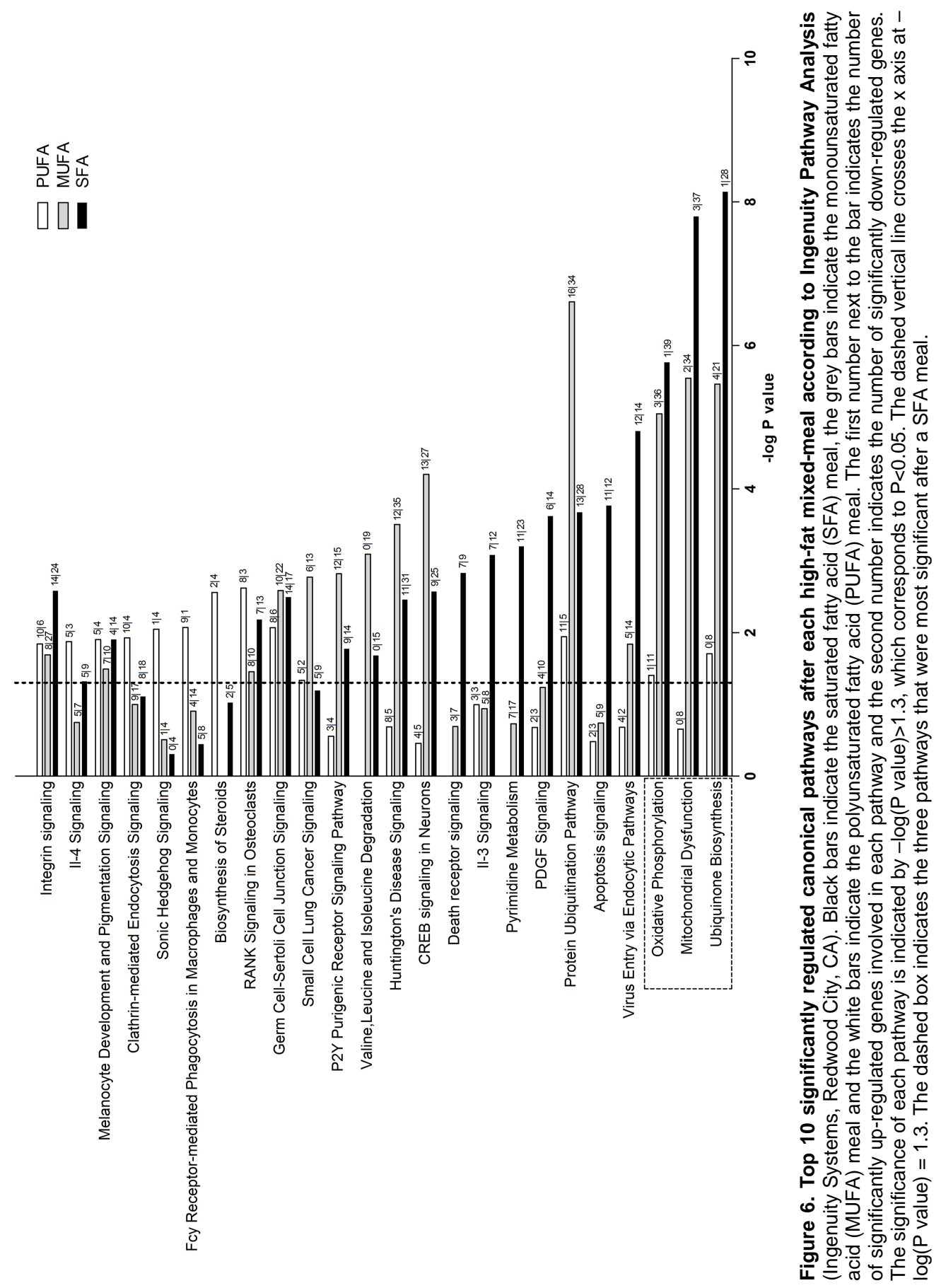




\section{Discussion}

The present study examined postprandial skeletal muscle FA handling in obese insulin resistant men consuming three high-fat mixed-meals with different FA composition. Ingestion of the PUFA meal resulted in an improved postprandial insulin sensitivity compared with SFA, which was accompanied by a reduced muscle TAG-derived FA uptake, and a tendency towards increased intramuscular lipid turnover.

Arterialized plasma insulin and glucose concentrations were higher after the SFA meal as compared with the PUFA meal, with intermediate values for the MUFA meal. Since there were no differences in net glucose flux between meals, this implies higher postprandial insulin sensitivity after the PUFA meal (as evidenced by data of the PGI). Our data are in line with previous studies, showing that a PUFA meal improved insulin sensitivity as compared with a SFA meal $(38,39)$, We found that the effect of MUFA was intermediate between the SFA meal and the PUFA meal, which is in agreement with studies that have shown beneficial effects of MUFA on insulin sensitivity as compared with SFA (39-42).

This differential effect on insulin sensitivity of PUFA versus SFA was accompanied by differences in skeletal muscle FA metabolism. The uptake of TAG-derived FA by forearm muscle decreased after the PUFA meal in the postprandial period as compared with the SFA and MUFA meal. Circulating TAG concentrations were not significantly lower after the PUFA meal compared to the other meals, suggesting that a reduced TAG supply is not responsible for the reduced PUFA-induced TAG extraction. The reduced TAG extraction may be related to the decreased postprandial insulin concentrations, which may have resulted in reduced muscle lipoprotein lipase (LPL) activity (35). Furthermore, the FA transporter CD36 is acutely upregulated during insulin stimulation (34). Thus, reduced CD36 content in skeletal muscle may also have contributed to the lower TAG extraction after the PUFA meal.

Beside the reduced uptake of TAG-derived FA with PUFA, it seemed that there was a higher fractional synthetic rate of the TAG, DAG and PL pool after the MUFA meal compared with the SFA meal. The fractional synthetic rate of the lipid pools was comparable after the PUFA and MUFA meal but values after the PUFA meal were not significantly different from the SFA meal. These data should be interpreted with caution, since skeletal muscle biopsies were taken $4 \mathrm{~h}$ hours after ingestion of the high-fat mixed-meal, when a steady-state has not yet been reached. Nevertheless, these data hint towards a higher incorporation of dietary [U$\left.{ }^{13} \mathrm{C}\right]$-labeled palmitate from the muscle FFA pool in the different lipid fractions in skeletal muscle with unsaturated FA compared with SFA. Additionally, there was a lesser down-regulation of the mitochondrial oxidative genes after the PUFA 
compared with the SFA or MUFA meal, suggesting that overall lipid turnover is higher after the PUFA compared with the SFA meal. The finding that a PUFAinduced improvement in postprandial insulin sensitivity is accompanied by a higher intramuscular lipid turnover is consistent with a recent study, demonstrating that obese prediabetic insulin resistant subjects have a reduced fractional synthetic rate of intramuscular TAG compared with controls (43). Overall, our data support the concept that not the TAG concentration per se but rather lipid turnover may determine insulin sensitivity.

Human studies examining the short-term effect of dietary FA on postprandial lipid profile suggest that the FA composition of the meal may influence the absorption, synthesis, and secretion of dietary TAG as well as the size of the chylomicron particles (44-46). This study showed that postprandial circulating chylomicron concentrations were highest after the MUFA meal. Other studies also found increased TAG concentration after a MUFA meal (44, 47-49). De Bruin et al. (48) found that the removal rate of olive oil chylomicron remnants was lower, which may be explained by a reduced endothelial LPL action due to their TAG content or greater particle number. This may imply that MUFA-chylomicrons have to rely on an alternative route for their removal from the circulation. Indeed, it has been suggested that hepatic lipase plays a significant role in the removal of MUFAenriched particles $(48,50)$.

It should be noted that we measured responses in the acute postprandial period. It remains to be established if improvement in insulin sensitivity after the PUFA meal due to lower lipid uptake (and possible less accumulation of fat in the muscle) is relevant in the acute postprandial period. In fact, the increase in insulin sensitivity after the PUFA meal may also be explained by other mechanisms beside lower lipid uptake. For example, differences in the secretion of gut hormones (glucagonlike peptide 1 and cholecystokinin) could potentiate differential insulin secretion and insulin sensitivity according to FA composition of the meals $(41,46)$. Thus, the exact mechanisms responsible for the differential postprandial insulin sensitivity should be elucidated in future studies. Furthermore, it remains to be established whether these acute effects may contribute to findings after long-term dietary intervention. It has become clear from dietary intervention studies that long-term isocaloric replacement of SFA has no major effects on insulin sensitivity (51).

In conclusion, the present study demonstrated that dietary FA have an acute effect on postprandial insulin sensitivity. Replacing SFA with PUFA may induce a reduced TAG-derived FA uptake, and a tendency toward a higher lipid turnover as reflected by a more transcriptional oxidative phenotype and altered intracellular lipid partitioning. These data suggest that the effects of replacement of SFA by PUFA may contribute to lower uptake of lipids in skeletal muscle and may be therefore protective against the development of insulin resistance in humans. 


\section{Acknowledgements}

We thank all subjects for participation in the present study. We gratefully thank Jos Stegen, Wendy Sluijsmans, Hasibe Aydeniz, Yvonne Essers and Annemie Gijssen for their excellent analytical support. The authors' responsibilities were as follows - EB designed research; AJ, EK, GG, FB and CM conducted research; AJ, EK, $M B$, and $L A$ analyzed data and performed statistical analysis; $A J$ and $E K$ wrote the paper; EB had primary responsibility for the final content; GG, FB, CM, MB, LA, $M M, E M$ and EB reviewed/edited the manuscript. There were no conflicts of interest.

This study was supported by a grant from the Dutch Diabetes Research Foundation (project number 2006.00.019) and the NutriGenomics Consortium within the Top Institute Food and Nutrition. The Scientific board of the Dutch Diabetes Research Foundation reviewed the initial research proposal and the NutriGenomics Consortium was involved in the microarray analysis. 


\section{References}

1. Goossens GH. The role of adipose tissue dysfunction in the pathogenesis of obesity-related insulin resistance. Physiol Behav 2008;94(2):206-18.

2. Frayn KN. Adipose tissue as a buffer for daily lipid flux. Diabetologia 2002;45(9):1201-10.

3. Blaak EE, Wagenmakers AJ, Glatz JF, et al. Plasma FFA utilization and fatty acid-binding protein content are diminished in type 2 diabetic muscle. Am $\mathrm{J}$ Physiol Endocrinol Metab 2000;279(1):E146-54.

4. Mensink M, Blaak EE, van Baak MA, Wagenmakers AJ, Saris WH. Plasma free Fatty Acid uptake and oxidation are already diminished in subjects at high risk for developing type 2 diabetes. Diabetes 2001;50(11):2548-54.

5. Kelley DE, Goodpaster B, Wing RR, Simoneau JA. Skeletal muscle fatty acid metabolism in association with insulin resistance, obesity, and weight loss. Am J Physiol 1999;277(6 Pt 1):E1130-41.

6. van Hees AM, Jans A, Hul GB, Roche HM, Saris WH, Blaak EE. Skeletal Muscle Fatty Acid Handling in Insulin Resistant Men. Obesity (Silver Spring) 2011.

7. Corcoran MP, Lamon-Fava S, Fielding RA. Skeletal muscle lipid deposition and insulin resistance: effect of dietary fatty acids and exercise. Am J Clin Nutr 2007;85(3):662-77.

8. Schmitz-Peiffer C. Signalling aspects of insulin resistance in skeletal muscle: mechanisms induced by lipid oversupply. Cell Signal 2000;12(9-10):583-94.

9. Schrauwen P, Hesselink MK. Oxidative capacity, lipotoxicity, and mitochondrial damage in type 2 diabetes. Diabetes 2004;53(6):1412-7.

10. Roden M. How free fatty acids inhibit glucose utilization in human skeletal muscle. News Physiol Sci 2004;19:92-6.

11. Mensink M, Blaak EE, Vidal H, De Bruin TW, Glatz JF, Saris WH. Lifestyle changes and lipid metabolism gene expression and protein content in skeletal muscle of subjects with impaired glucose tolerance. Diabetologia 2003;46(8):1082-9.

12. Mensink M, Hesselink MK, Russell AP, Schaart G, Sels JP, Schrauwen P. Improved skeletal muscle oxidative enzyme activity and restoration of PGC-1 alpha and PPAR beta/delta gene expression upon rosiglitazone treatment in obese patients with type 2 diabetes mellitus. Int $\mathrm{J}$ Obes (Lond) 2007;31(8):1302-10.

13. Corpeleijn E, Feskens EJ, Jansen EH, et al. Improvements in glucose tolerance and insulin sensitivity after lifestyle intervention are related to changes in serum fatty acid profile and desaturase activities: the SLIM study. Diabetologia 2006;49(10):2392-401.

14. Hanada H, Morikawa K, Hirota K, Nonaka M, Umehara Y. Induction of apoptosis and lipogenesis in human preadipocyte cell line by n-3 PUFAs. Cell Biol Int 2011;35(1):51-9.

15. Montell E, Turini M, Marotta $M$, et al. DAG accumulation from saturated fatty acids desensitizes insulin stimulation of glucose uptake in muscle cells. Am J Physiol Endocrinol Metab 2001;280(2):E229-37.

16. Gaster M, Rustan AC, Beck-Nielsen H. Differential utilization of saturated palmitate and unsaturated oleate: evidence from cultured myotubes. Diabetes 2005;54(3):648-56.

17. Roche HM. Fatty acids and the metabolic syndrome. Proc Nutr Soc 2005;64(1):23-9.

18. Bickerton AS, Roberts R, Fielding BA, et al. Preferential uptake of dietary Fatty acids in adipose tissue and muscle in the postprandial period. Diabetes 2007;56(1):168-76.

19. DeFronzo RA, Tobin JD, Andres R. Glucose clamp technique: a method for quantifying insulin secretion and resistance. Am J Physiol 1979;237(3):E214-23.

20. van Hees AM, Saris WH, Hul GB, et al. Effects of dietary fat modification on skeletal muscle fatty acid handling in the metabolic syndrome. Int J Obes (Lond) 2010;34(5):859-70.

21. Goossens GH, Blaak EE, Schiffers PM, Saris WH, van Baak MA. Effect of short-term ACE inhibitor treatment on peripheral insulin sensitivity in obese insulin-resistant subjects. Diabetologia 2006;49(12):3009-16.

22. Adriaens MP, Schoffelen PF, Westerterp KR. Intra-individual variation of basal metabolic rate and the influence of daily habitual physical activity before testing. Br J Nutr 2003;90(2):41923.

23. Schoffelen PF, Westerterp KR, Saris WH, Ten Hoor F. A dual-respiration chamber system with automated calibration. J Appl Physiol 1997;83(6):2064-72.

24. Weir JB. New methods for calculating metabolic rate with special reference to protein metabolism. J Physiol 1949;109(1-2):1-9.

25. Bergstrom J, Hermansen L, Hultman E, Saltin B. Diet, muscle glycogen and physical performance. Acta Physiol Scand 1967;71(2):140-50. 
26. Hoffman E. Expression profiling--best practices for data generation and interpretation in clinical trials. Nature reviews 2004;5(3):229-37.

27. Dai $M$, Wang $P$, Boyd $A D$, et al. Evolving gene/transcript definitions significantly alter the interpretation of GeneChip data. Nucleic acids research 2005;33(20):e175.

28. Irizarry RA, Bolstad BM, Collin F, Cope LM, Hobbs B, Speed TP. Summaries of Affymetrix GeneChip probe level data. Nucleic acids research 2003;31(4):e15.

29. Bolstad BM, Irizarry RA, Astrand M, Speed TP. A comparison of normalization methods for high density oligonucleotide array data based on variance and bias. Bioinformatics (Oxford, England) 2003;19(2):185-93.

30. Storey JD, Tibshirani R. Statistical significance for genomewide studies. Proceedings of the National Academy of Sciences of the United States of America 2003;100(16):9440-5.

31. Smyth GK. Linear models and empirical bayes methods for assessing differential expression in microarray experiments. Statistical applications in genetics and molecular biology 2004;3:Article3.

32. Vandesompele J, De Preter K, Pattyn F, et al. Accurate normalization of real-time quantitative RT-PCR data by geometric averaging of multiple internal control genes. Genome biology 2002;3(7):RESEARCH0034.

33. Matthews DR, Hosker JP, Rudenski AS, Naylor BA, Treacher DF, Turner RC. Homeostasis model assessment: insulin resistance and beta-cell function from fasting plasma glucose and insulin concentrations in man. Diabetologia 1985;28(7):412-9.

34. Bonen A, Benton CR, Campbell SE, et al. Plasmalemmal fatty acid transport is regulated in heart and skeletal muscle by contraction, insulin and leptin, and in obesity and diabetes. Acta Physiol Scand 2003;178(4):347-56.

35. Wang $\mathrm{H}$, Eckel RH. Lipoprotein lipase: from gene to obesity. Am J Physiol Endocrinol Metab 2009;297(2):E271-88.

36. RR W. Radioactive and Stable Isotope Tracers in Biomedicine: Principles and Practice of Kinetics Analysis.: Wiley-Liss: New York, USA, 1992.

37. Guo Z, Jensen MD. Intramuscular fatty acid metabolism evaluated with stable isotopic tracers. J Appl Physiol 1998;84(5):1674-9.

38. Xiao C, Giacca A, Carpentier A, Lewis GF. Differential effects of monounsaturated, polyunsaturated and saturated fat ingestion on glucose-stimulated insulin secretion, sensitivity and clearance in overweight and obese, non-diabetic humans. Diabetologia 2006;49(6):13719.

39. Lopez S, Bermudez B, Pacheco YM, Villar J, Abia R, Muriana FJ. Distinctive postprandial modulation of beta cell function and insulin sensitivity by dietary fats: monounsaturated compared with saturated fatty acids. Am J Clin Nutr 2008;88(3):638-44.

40. Lopez S, Bermudez B, Ortega A, et al. Effects of meals rich in either monounsaturated or saturated fat on lipid concentrations and on insulin secretion and action in subjects with high fasting triglyceride concentrations. Am J Clin Nutr 2011;93(3):494-9.

41. Robertson MD, Jackson KG, Fielding BA, Williams CM, Frayn KN. Acute effects of meal fatty acid composition on insulin sensitivity in healthy post-menopausal women. $\mathrm{Br} J$ Nutr 2002;88(6):635-40.

42. Paniagua JA, de la Sacristana AG, Sanchez E, et al. A MUFA-rich diet improves posprandial glucose, lipid and GLP-1 responses in insulin-resistant subjects. J Am Coll Nutr 2007;26(5):434-44.

43. Perreault L, Bergman BC, Hunerdosse DM, Playdon MC, Eckel RH. Inflexibility in intramuscular triglyceride fractional synthesis distinguishes prediabetes from obesity in humans. Obesity (Silver Spring) 2010;18(8):1524-31.

44. Jackson KG, Wolstencroft EJ, Bateman PA, Yaqoob P, Williams CM. Acute effects of meal fatty acids on postprandial NEFA, glucose and apo E response: implications for insulin sensitivity and lipoprotein regulation? Br J Nutr 2005;93(5):693-700.

45. Weintraub MS, Zechner R, Brown A, Eisenberg S, Breslow JL. Dietary polyunsaturated fats of the W-6 and W-3 series reduce postprandial lipoprotein levels. Chronic and acute effects of fat saturation on postprandial lipoprotein metabolism. J Clin Invest 1988;82(6):1884-93.

46. Zampelas A, Peel AS, Gould BJ, Wright J, Williams CM. Polyunsaturated fatty acids of the n6 and $\mathrm{n}-3$ series: effects on postprandial lipid and apolipoprotein levels in healthy men. Eur $\mathrm{J}$ Clin Nutr 1994;48(12):842-8.

47. Mekki N, Charbonnier M, Borel $\mathrm{P}$, et al. Butter differs from olive oil and sunflower oil in its effects on postprandial lipemia and triacylglycerol-rich lipoproteins after single mixed meals in healthy young men. J Nutr 2002;132(12):3642-9. 
48. de Bruin TW, Brouwer CB, van Linde-Sibenius Trip M, Jansen H, Erkelens DW. Different postprandial metabolism of olive oil and soybean oil: a possible mechanism of the highdensity lipoprotein conserving effect of olive oil. Am J Clin Nutr 1993;58(4):477-83.

49. Higashi K, Ishikawa T, Shige H, et al. Olive oil increases the magnitude of postprandial chylomicron remnants compared to milk fat and safflower oil. J Am Coll Nutr 1997;16(5):42934.

50. Brouwer CB, de Bruin TW, Jansen H, Erkelens DW. Different clearance of intravenously administered olive oil and soybean-oil emulsions: role of hepatic lipase. Am J Clin Nutr 1993;57(4):533-9.

51. Tierney AC, McMonagle J, Shaw DI, et al. Effects of dietary fat modification on insulin sensitivity and on other risk factors of the metabolic syndrome-LIPGENE: a European randomized dietary intervention study. Int J Obes (Lond) 2010. 


\section{6}

\section{The effect of polydextrose and soluble corn fiber on}

\section{energy metabolism, metabolic profile and appetite control in overweight men and women}

Ellen Konings

Paul F. Schoffelen Jos Stegen

Ellen E. Blaak 


\section{Abstract}

Background: High fiber diets offer several beneficial health effects.

Aim: The objective of the present study was to investigate whether replacement of $30 \%$ of available carbohydrates with polydextrose (PDX) or soluble corn fiber (SCF) at breakfast and lunch would result in an increased fat oxidation rate and satiety, which may be of relevance for body weight control and diabetes prevention. Methods: In a single-blind randomized crossover study, 18 overweight men and women underwent four different dietary interventions, which consisted of a PDX diet, SCF diet and two control diets (full caloric and iso-caloric, comparable with PDX with respect to grams or energy\% of macronutrients, respectively). Glycemic profile, energy expenditure and substrate oxidation were measured for 24 hours in a respiration chamber. Circulating insulin, free fatty acids (FFA) and triacylglycerols were determined over a 14-hour period during daytime. Appetite ratings were assessed with visual analogue scales.

Results: Replacement of available carbohydrates with PDX or SCF reduced the peak glucose response, which was accompanied by reduced postprandial insulin responses. Moreover, higher levels of circulating FFA were observed after both fiber diets, which was accompanied by an increased fat oxidation over 24 hours. This effect was mainly attributed by the lower caloric value in the fiber diets and not by the fibers per se. Beside the increase in fat oxidation there was a pronounced suppressive effect on appetite ratings by PDX.

Conclusion: Replacing available carbohydrates by PDX may be of special interest because of their beneficial effects on metabolic profile and may affect body weight control in the long term. 


\section{Introduction}

The prevalence of obesity has increased rapidly worldwide and the importance of developing dietary strategies to tackle the problem is widely acknowledged $(1,2)$. Although it is well known that excess calorie intake is the primary dietary cause, alterations in food patterns or nutrients must be considered (3). Consumption of diets high in dietary fiber has been reported to result in a number of beneficial health effects including a reduced risk of cardiovascular disease, diabetes, hypertension, obesity and gastrointestinal disorders (4). High dietary fiber intake may prevent weight gain by reducing appetite and energy intake $(5,6)$. Dietary fibers may decrease intestinal passage time, leading to a more gradual nutrient absorption and prolonged feelings of satiety. The slower nutrient uptake may result in reduced postprandial glucose and insulin responses (7-11). The lower glucose and insulin concentrations may, in turn result in a reduced inhibition of lipolysis, higher circulating free fatty acid (FFA) concentrations and consequently increase fat oxidation $(12,13)$. Additionally, dietary fibers can be fermented in the colon, with the production of short chain fatty acids (SCFA) (acetate, butyrate and propionate) and gases (hydrogen, methane and carbon dioxide), which may enhance satiety and affect energy metabolism via various mechanisms (14). Epidemiological evidence of these health-protective effects of dietary fiber has led to industrial interest in developing novel dietary fibers that can be incorporated in a variety of food products to increase fiber content. The question remains whether these functional soluble fibers provide the same health benefits associated with 'traditional' dietary fibers. Only few soluble fibers can be used to be incorporated in food products and beverages. The use of Polydextrose (PDX) and Soluble Corn Fiber (SCF), also known as Soluble Gluco Fiber in Europe, seems a good option since they have a higher digestive tolerance compared with other soluble fibers, like for example inulin (15-17). PDX has randomly polymerized branched chains and various types of glycosidic bonds (18). SCF is a fiber obtained from corn starch, comprised of glucose oligosaccharides with digestion resistant glycosidic linkages and contains minor amounts of mono and disaccharides (19). Both fibers are poorly digested in the small intestine, but are partially fermented by the gut microbiota (20). We hypothesized that replacement of available carbohydrates with PDX or SCF may result in a reduced inhibition of postprandial fat oxidation rate and increased satiety mediated through differences in glycemic and insulinemic and FFA responses. A higher postprandial fat oxidation may have an impact on fat storage and satiety, and may thereby translate in a negative energy balance in the long term (21-23). Secondly, the higher postprandial fat oxidation may result in less lipid accumulation in non-adipose tissues, thereby improving insulin sensitivity and the metabolic profile in the longer term $(24,25)$. In the present study, we studied 
replacement of $30 \%$ of the total daily intake of available carbohydrates by PDX or SCF provided at breakfast and lunch, on glycemic and insulinemic profile, energy expenditure and substrate oxidation over a period of 24 hours.

\section{Methods}

\section{Subjects}

Eighteen overweight subjects ( 9 men and 9 women) between 20 and 50 years of age participated in a single-blind randomized cross-over study. The men and women were matched for age and body mass index (BMI) (Table 1). Additional inclusion criteria were fasting plasma glucose $<6.1 \mathrm{mmol} / \mathrm{L}$ and a stable body weight in the last 3 months (weight gain or loss $<3 \mathrm{~kg}$ ). None of the subjects were on medication (except contraception) or were engaged in sports activities for more than three hours per week. Body composition was determined by bio-impedance (Bodystat Quadscan 4000, Bodystat Ltd, Douglas, DQ, British Isles). The study protocol was reviewed and approved by the Medical Ethical Committee of Maastricht University Medical Centre (MUMC+) and all subjects gave their written informed consent before participating in the study.

Table 1. Subject characteristics.

\begin{tabular}{lccc}
\hline & men $(\mathbf{n}=9)$ & women $(\mathbf{n}=\mathbf{9})$ & p-value \\
\hline Age (years) & $32.4 \pm 2.7$ & $33.1 \pm 3.5$ & 0.883 \\
Body weight (kg) & $86.6 \pm 2.3$ & $81.1 \pm 2.2$ & 0.104 \\
BMI (kg/m $\left.{ }^{2}\right)$ & $27.3 \pm 0.5$ & $27.2 \pm 0.4$ & 0.910 \\
Body fat (\%) & $18.4 \pm 1.3$ & $29.9 \pm 2.0$ & 0.000 \\
Fat free mass (kg) & $70.7 \pm 2.4$ & $56.8 \pm 2.0$ & 0.000 \\
Fat mass (kg) & $15.9 \pm 1.0$ & $24.3 \pm 1.8$ & 0.001 \\
Waist-hip ratio & $1.00 \pm 0.01$ & $0.91 \pm 0.01$ & 0.000 \\
SBP (mmHg) & $121 \pm 3$ & $111 \pm 3$ & 0.051 \\
DBP (mmHg) & $77 \pm 2$ & $72 \pm 1$ & 0.028 \\
FPG (mmol/l) & $5.1 \pm 0.1$ & $5.1 \pm 0.2$ & 0.932 \\
HbA1c & $5.4 \pm 0.1$ & $5.3 \pm 0.1$ & 0.352
\end{tabular}

Values are mean \pm SEM. Men and women were compared using a 2-tailed Student's t test for unpaired samples. BMI, body mass index; SBP, systolic blood pressure; DBP, diastolic blood pressure; FPG, fasting plasma glucose.

\section{Study Design}

The design of the study was a randomized single-blind cross-over design with four different dietary interventions, including two fiber diets with polydextrose (PDX) or Soluble Corn Fiber (SCF) also known as Soluble Gluco Fiber in Europe and two control diets (full caloric and iso-caloric), with a 1 week washout period between diets (see under Diet composition). The subjects were instructed not to change 
their lifestyle and dietary habits during the washout period. Before each test period, subjects received a standardized meal $(2385 \mathrm{~kJ})$ at $19 \mathrm{~h} 00$ the evening before they entered the respiration chamber. Each respiration chamber visit started after the continuous glucose monitoring system (CGMS) (Medtronic, MiniMed Inc., Sylmar, CA, USA) was inserted in the peri-umbelical region at the abdomen of the subject at $20 \mathrm{~h} 00$ and ended 36 hours later at $8 \mathrm{~h} 00$. The CGMS was calibrated four times per day against finger-stick glucose determinations. In the first 12 hours, the subjects could accustom to the chamber and during the first night, the sleeping metabolic rate (SMR) was assessed to estimate total energy intake. The SMR was calculated as the lowest energy expenditure (EE) (measured in 30 minutes intervals) over three consecutive sleeping hours (26). Based on the SMR (multiplied with a physical activity index of 1.55 (27)), total energy intake was estimated. Subjects were fed in energy balance in all situations, since the difference between 24 hour EE and energy intake was less than $10 \%$ deviation (27). For standardization of the measurements, the humidity in the respiration chamber was fixed, clothing was standardized and physical activity was prescribed by means of a standardized physical activity protocol (28). In the morning at $7 \mathrm{~h} 30$ an indwelling cannula was inserted into an anticubital vein for the withdrawal of the first fasting blood samples. Thereafter, subjects received one of the four different diets in randomized order. During the day they received a breakfast at $8 \mathrm{~h}$, a lunch at $12 \mathrm{~h}$ and dinner at $17 \mathrm{~h} 30$ and three snacks at $10 \mathrm{~h}, 15 \mathrm{~h} 30$ and $20 \mathrm{~h} 30$. Water and tea (except green tea) were available ad libitum. Profile of glucose, FFA, triacylglycerols (TAG) and insulin were determined over a 14-hour period during daytime from $8 \mathrm{~h} 00$ until $21 \mathrm{~h} 30$. Blood sampling was performed immediately before ingestion of the three meals (breakfast, lunch and dinner) at $\mathrm{t}=0 \mathrm{~min}$, and 30,60 , 120 and 240 minutes postprandially. The hydrogen concentration was measured in end-expiration (alveolar) breath samples every $2 \mathrm{~h}$ from $8 \mathrm{~h} 00$ until $22 \mathrm{~h} 00$ and again at 8 h00 the next morning using a gastrolyzer ${ }^{\circledR}$ (Bedfont Scientific Ltd., The Netherlands), as a marker for colonic fermentation. Visual analogue scales and well-being questionnaires were completed 19 times throughout the test day as described in detail below.

\section{Diet composition}

The four different dietary interventions consisted of two fiber diets (Stalite ${ }^{\circledR}$ Polydextrose (PDX) and Promitor ${ }^{\mathrm{TM}}$ Soluble Corn Fiber (SCF), manufactured by Tate \& Lyle) and two control diets (full caloric and iso-caloric). In the PDX and SCF diet, $30 \%$ of the available carbohydrates at breakfast and lunch of the full caloric control diet were replaced by respectively PDX $(56.7 \pm 0.9 \mathrm{~g} /$ day $)$ or SCF $(54.6 \pm 0.9$ $\mathrm{g} /$ day). Logically, both fiber diets had a lower caloric value than the full caloric control diet. To correct for the lower caloric value we also compared the PDX diet 
with a second control diet with the same caloric intake as the PDX diet (iso-caloric control). The full caloric control diet, PDX and SCF diet consisted of approximately the same macronutrient composition in grams. The iso-caloric control diet and PDX diet had approximately the same macronutrient composition in energy percentage (en\%) (Table 2). The food products consisted of test products provided by Tate and Lyle (Innovation Centre, France) and fresh and packed products bought at a convenience store in the Netherlands. The test products provided by Tate and Lyle included breakfast cereals, pudding and two instant drinks (powder mix for a hot cocoa instant drink and a powder mix for an orange drink), all containing PDX or SCF. In the full caloric and iso-caloric diet, placebo test products were given which consist of full available carbohydrates with no PDX or SCF added. The fresh and packed products consisted of semi-skimmed and full milk, high fat quark (type of yoghurt), wheat bread, cheese, salami, margarine, apples, spaghetti bolognese and salted peanuts. An overview of the diet composition is given in table 2. Besides the fiber test products, which were only given in the PDX and SCF condition, the remainder of the diet consisted of $19.1 \pm 0.3 \mathrm{~g}$ fiber in the full caloric condition, 16.0 $\pm 0.3 \mathrm{~g}$ fiber in the iso-caloric condition and $14.6 \pm 0.2 \mathrm{~g}$ fiber in both the PDX and SCF condition.

Table 2. Diet composition.

\begin{tabular}{lcccc}
\hline & Iso-caloric diet & Full caloric diet & PDX diet & SCF diet \\
\hline Energy (MJ) & $9.5 \pm 0.2$ & $10.4 \pm 0.2$ & $9.5 \pm 0.2$ & $9.7 \pm 0.2$ \\
Energy (kcal) & $2261.2 \pm 35.3$ & $2489.3 \pm 38.9$ & $2261.2 \pm 35.3$ & $2323.8 \pm 36.3$ \\
Protein (En\%) & $15.4 \pm 0.2$ & $14.9 \pm 0.2$ & $16.4 \pm 0.3$ & $15.9 \pm 0.2$ \\
Protein (g/day) & $87.0 \pm 1.4$ & $92.7 \pm 1.5$ & $92.9 \pm 1.5$ & $92.5 \pm 1.5$ \\
Carbohydrate (En\%) & $46.5 \pm 0.7$ & $49.7 \pm 0.8$ & $46.5 \pm 0.7$ & $48.1 \pm 0.8$ \\
Carbohydrate & $262.7 \pm 4.1$ & $309.3 \pm 4.8$ & $249.4 \pm 3.9$ & $252.8 \pm 4.0$ \\
$\quad$ PDX (g/day) & $/$ & $/$ & $56.7 \pm 0.9$ & $/$ \\
$\quad$ SCF (g/day) & $/$ & $/$ & $/$ & $54.6 \pm 0.9$ \\
Fat (En\%) & $39.2 \pm 0.6$ & $35.8 \pm 0.6$ & $39.5 \pm 0.6$ & $38.3 \pm 0.6$ \\
Fat (g/day) & $98.5 \pm 1.5$ & $99.1 \pm 1.6$ & $99.2 \pm 1.6$ & $98.9 \pm 1.5$ \\
\hline
\end{tabular}

Values are mean \pm SEM.

\section{4h energy expenditure (EE)}

The 24h EE and substrate oxidation as well as the pattern of these parameters over day and night were calculated from $7 \mathrm{~h} 30$ to $7 \mathrm{~h} 30$. Metabolic rate was calculated from VO2 $(1 / \mathrm{min})$ and VCO2 $(1 / \mathrm{min})$ according to the equations of Frayn (29). Energy expenditure was calculated using the formula of Weir (30). During the stay in the respiration chamber 24-hour urine was collected from 8 h00 to 8 h00. Subjects emptied their bladder at $8 \mathrm{~h} 00$ before starting the collection. Urine volume and nitrogen concentration were measured, the latter using a nitrogen analyzer 
(Heraeus, type CHN-O-Rapid). SMR of the second night was defined as the lowest mean EE measured over three consecutive hours between 00h00 and 07h00 and was used in further calculation. Diet-induced thermogenesis (DIT) and activityinduced energy expenditure (AEE) were calculated as described previously (31).

\section{Questionnaires}

The Visual Analogue scale questionnaire (VAS, on a $100 \mathrm{~mm}$ scale) contains questions about feelings of hunger, fullness, satiety and desire to eat (32). Opposing extremes were described at either end of a $100 \mathrm{~mm}$ horizontal line and subjects marked the line to indicate how they felt at that moment. Well-being was determined using a questionnaire on the occurrence of complaints (nausea, abdominal bloating, stomach- intestinal cramps, diarrhea, and other symptoms). Symptoms were graded on a 10-point scale with grade 1 representing 'not present' to grade 10 'strongly present'. Subjects were asked to mark how they felt at the moment. For the analysis of the well-being questionnaire, a symptom was considered present if the grade was scored $>5$.

\section{Biochemical analysis}

Blood was collected in pre-cooled tubes containing 0.2M EDTA (Sigma, Dorset, UK). After collection, blood samples were centrifuged immediately at $4^{\circ} \mathrm{C}$ for 10 min at $1000 \mathrm{~g}$ and frozen at $-80^{\circ} \mathrm{C}$ until analysis. Plasma glucose, FFA and TAG were analyzed using standard enzymatic techniques automated on a Cobas Fara centrifugal spectrophotometer (Roche Diagnostics, Basel, Switzerland). Plasma insulin was measured with a double antibody radioimmunoassay (Linco Research, St Charles, MO).

\section{Statistical analysis}

Differences between the diets were examined using repeated-measures ANOVA. First, PDX was compared with the full and iso-caloric diet, thereafter SCF was compared with the PDX and full caloric diet. When a significant diet by time interaction was observed, LSD post-hoc testing was performed. Postprandial area under the curve (AUC) was calculated using the trapezoidal rule for all plasma metabolites, energy and substrate metabolism and appetite scores. Data are reported as mean \pm SEM. A P-Value $<0.05$ was considered statistically significant. Statistical analyses were performed using the statistical program SPSS 16.0 for Mac OS X. 


\section{Chapter 6}

\section{Results}

\section{Interstitial and plasma glucose and plasma insulin responses}

There were no differences in integrated interstitial glucose response between the four dietary interventions over 24 hours (Figure 1A). This was also reflected in plasma, since there were no differences in plasma glucose over the day (Figure 1B). The peak glucose response after breakfast was lower after both fiber diets compared with the full caloric diet (Full vs. PDX $p=0.06$, Full vs. SCF $p=0.03$ ). After lunch, the peak glucose response was lower after the SCF diet compared with the full caloric diet (Full vs. SCF $p=0.03$ ) (Figure 1B). During the night, PDX showed the highest glucose response ( $A \cup C$ night $p=0.02$ and $p=0.03$, post-hoc Full vs. PDX $p=0.007$, ISO vs. PDX $p=0.08$, SCF vs. PDX $p=0.05$ ) (Figure 1A). After breakfast and lunch, the insulin response was significantly lower in the PDX and SCF diet compared with the full caloric diet (AUC breakfast $(8 \mathrm{~h}-10 \mathrm{~h}) \mathrm{p}=0.04$ and $p=0.001$, post-hoc Full vs. PDX $p=0.02$, Full vs. SCF $p=0.003$; AUC lunch (12h14h) $p=0.04$ and $p=0.01$, post-hoc Full vs. $P D X p=0.03$, Full vs. SCF $p=0.01$ ). Over the whole day, the reduced insulin response was only significant for the SCF diet (Figure 2).

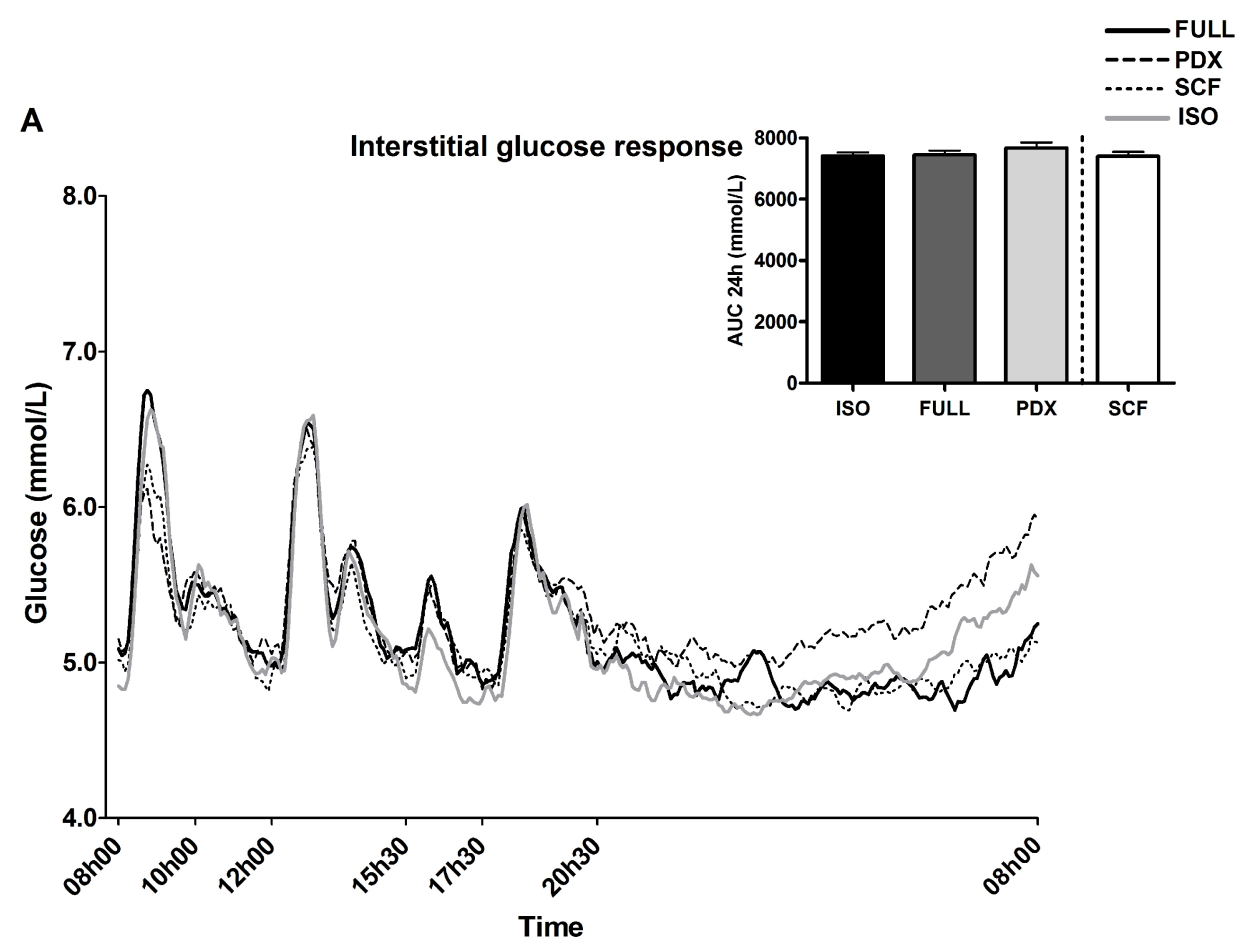




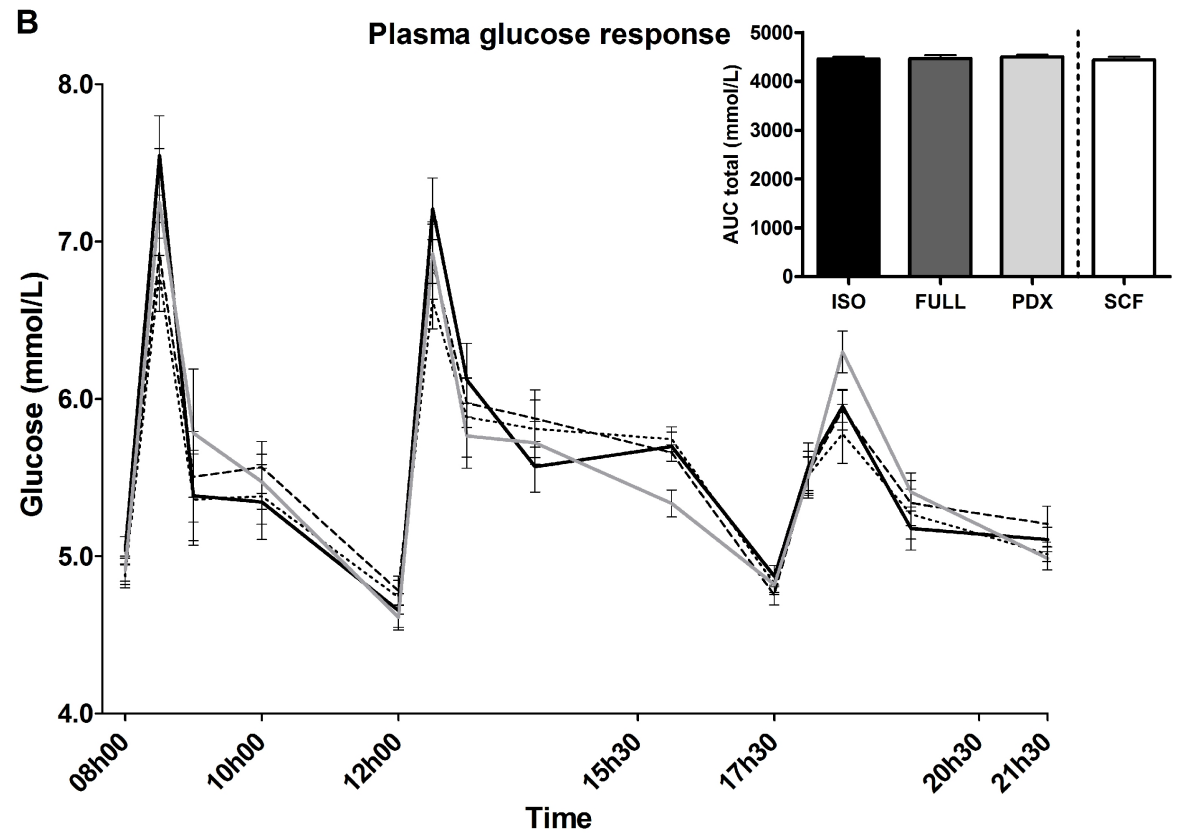

Figure 1. Postprandial glucose responses. (A) Interstitial glucose response measured with a continuous glucose monitoring system over 24 hours. (B) Plasma glucose response measured during daytime. Repeated measures ANOVA were performed with LSD post-hoc testing using the integrated responses $(A \cup C) . n=18$. Data are mean \pm SEM.

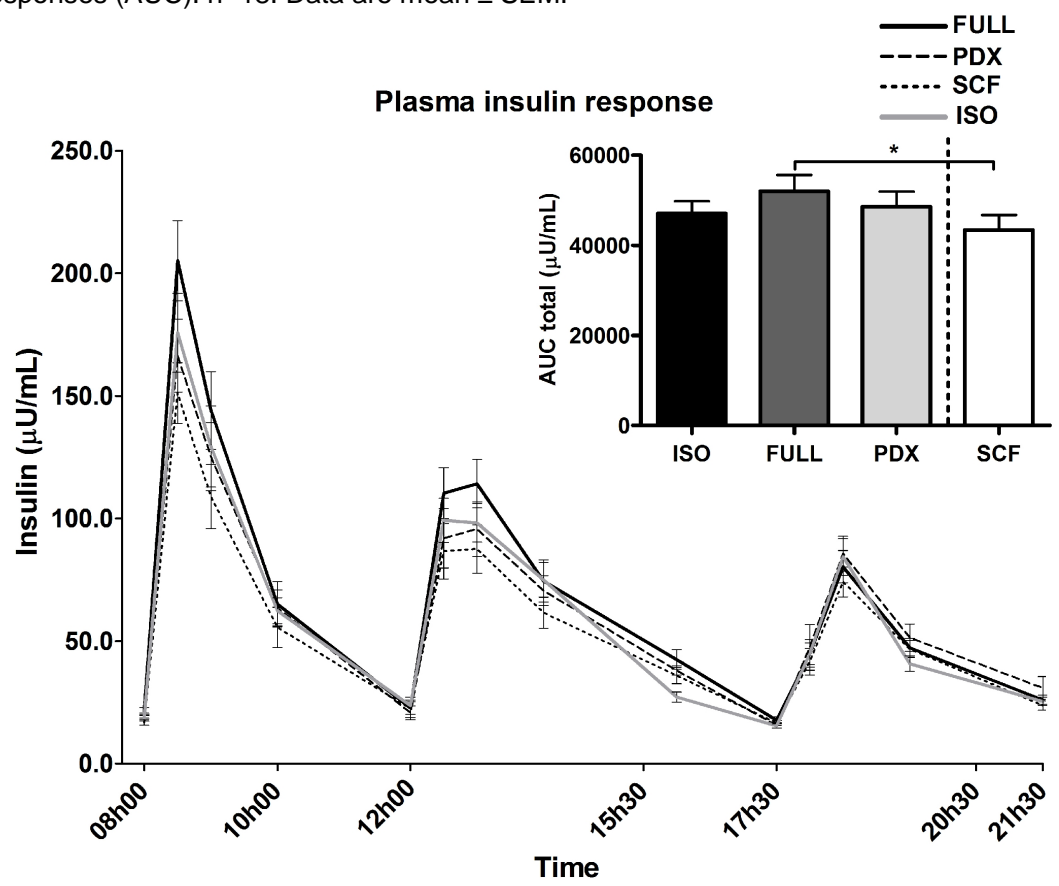

Figure 2. Postprandial insulin responses. Insulin response measured over time and AUC insulin measured during daytime. Repeated measures ANOVA were performed with LSD post-hoc testing using the integrated responses (AUC). $n=18$. Data are mean \pm SEM. * $p<0.05$. 


\section{Chapter 6}

\section{FFA and TAG responses}

During the day, the integrated FFA response was significantly higher in the PDX, SCF and iso-caloric diet compared with the full caloric diet indicating that the higher FFA response was due to the lower amount of calories in the diets rather than a specific response to PDX or SCF (Figure 3A-B). For both fiber diets, the lower concentrations of insulin in the postprandial state may reduce the inhibition of lipolysis and may explain the higher FFA response, which was observed. There were no differences in TAG response between all diets (Figure 3C-D).

A

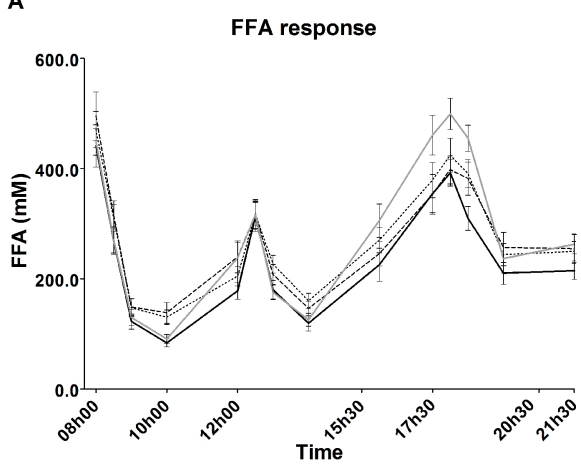

FFA

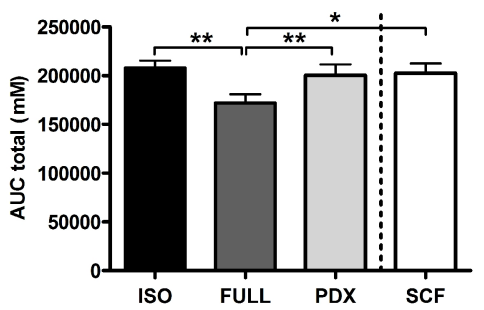

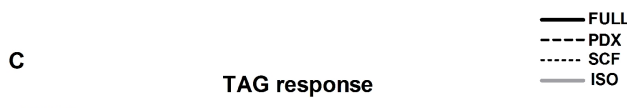

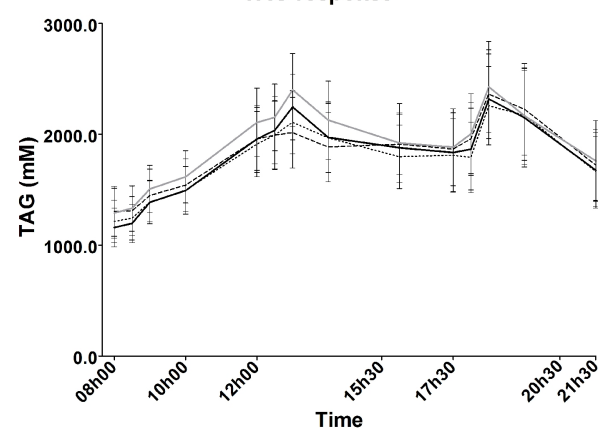

TAG

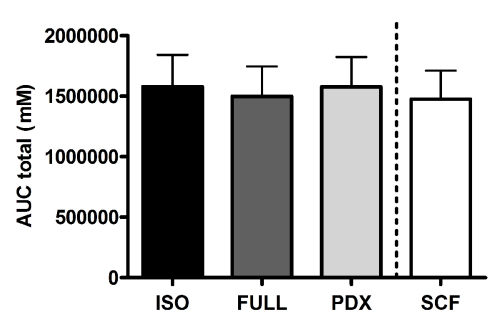

Figure 3. Postprandial FFA and TAG responses. (A) FFA response measured over time. (B) AUC FFA measured during daytime. (C) TAG response measured over time. (D) AUC TAG measured during daytime. Repeated measures ANOVA were performed with LSD post-hoc testing using the integrated responses $(A \cup C) . n=18$. Data are mean \pm SEM. * $p<0.05,{ }^{* *} p<0.01$.

\section{Energy expenditure}

Energy expenditure over 24 hours was not significantly different between the four diets (Figure 4A-B). There were also no differences in sleeping metabolic rate (SMR) during the second night, diet-induced thermogenesis (DIT) and activity induced energy expenditure (AEE) (Figure 4C-E). On average, subjects were in energy balance on the full caloric diet $(+0.19 \pm 0.17 \mathrm{MJ} / \mathrm{d}, \mathrm{p}=0.3)$ and were in negative energy balance on the PDX $(-0.83 \pm 0.17 \mathrm{MJ} / \mathrm{d}, \mathrm{p}=0.000)$, iso-caloric ($0.78 \pm 0.17 \mathrm{MJ} / \mathrm{d} \mathrm{p}=0.000)$ and the SCF diet $(-0.60 \pm 0.18 \mathrm{MJ} / \mathrm{d} \mathrm{p}=0.003)$. There was a significant difference in energy balance between the full caloric diet and the 
three other diets (iso-caloric, PDX and SCF diet) ( $p=0.000$, post-hoc Full vs. PDX: $p=0.000$, Full vs ISO: $p=0.000$, Full vs SCF: $p=0.000$ ). This may be explained by the lower amount of calories in both fiber diets and the iso-caloric diet.
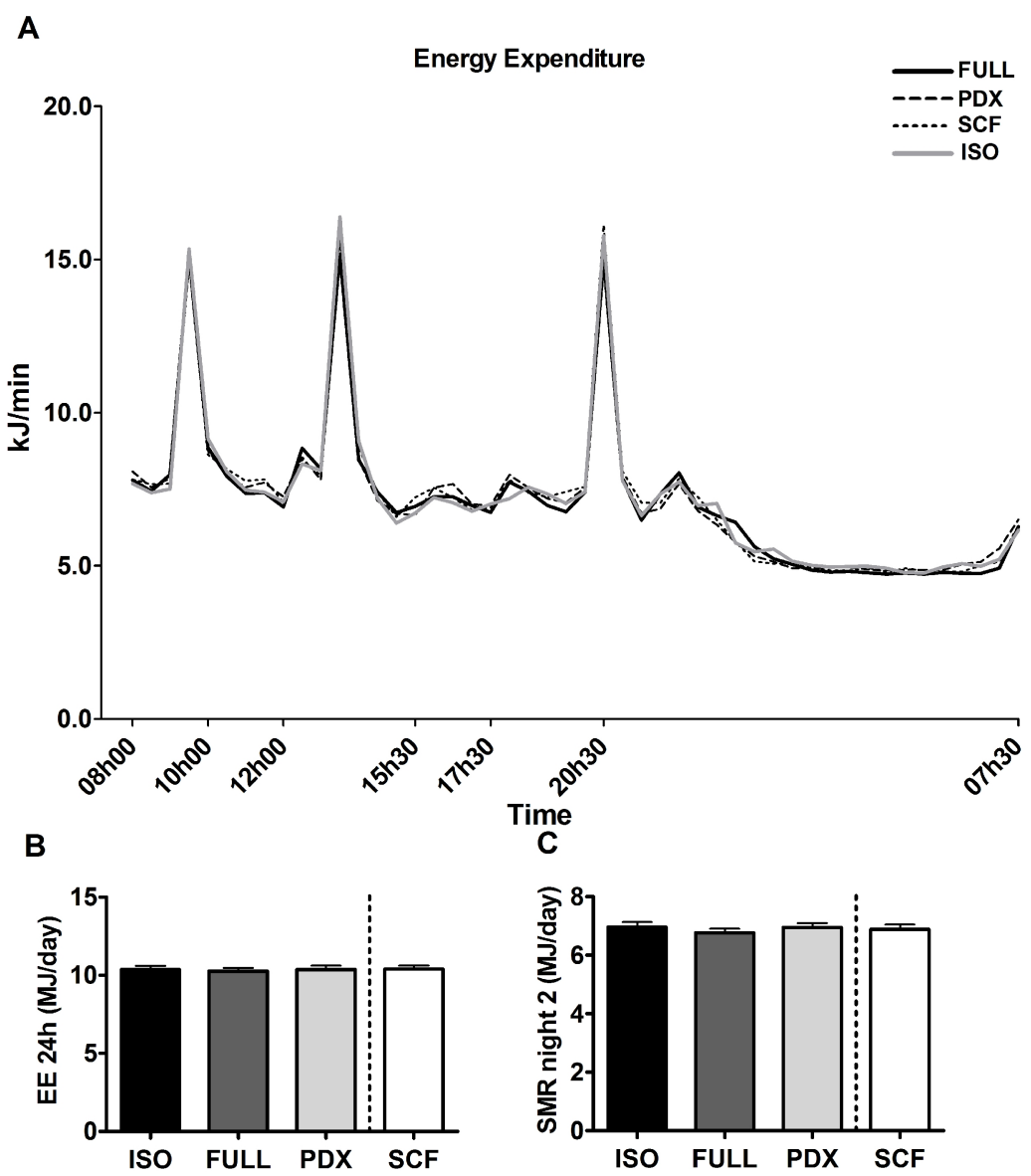

D

E
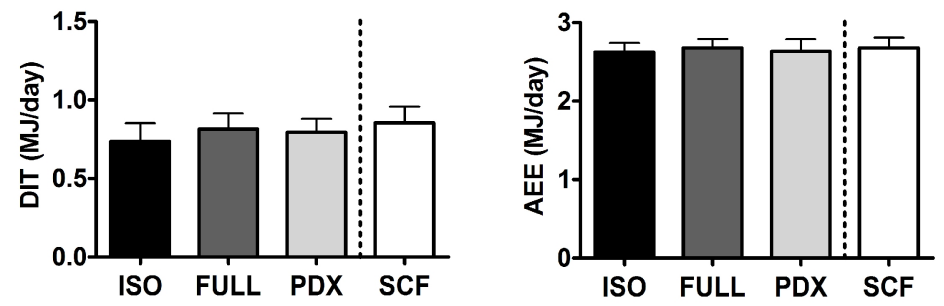

Figure 4. Energy expenditure measured over 24 hours in a respiration chamber. (A) Energy expenditure measured over time. (B) Energy expenditure measured during 24 hours. (C) Sleeping metabolic rate (SMR) of the second night measured during 24 hours. (D) Diet induced thermogenesis (DIT) measured during 24 hours. (E) Activity energy expenditure (AEE) measured during 24 hours. Repeated measures ANOVA were performed with LSD post-hoc testing. $n=16$. Data are mean \pm SEM. 


\section{Chapter 6}

\section{Substrate oxidation}

Over 24 hours, carbohydrate oxidation was reduced during the PDX and SCF diet as compared with the full caloric diet (Figure 5A-B). Fat oxidation was increased in both fiber diets and the iso-caloric diet as compared with the full caloric diet over 24 hours (Figure 6A-B). This indicates that the higher fat oxidation is due to the lower amount of calories in the diets rather than a specific response to PDX or SCF. The effect on substrate oxidation was mainly observed during the day (Figure $5 \mathrm{C}$ and 6C). During the night, the iso-caloric diet showed a lower carbohydrate oxidation and a higher fat oxidation as compared with the full caloric and PDX diet (Figure 5D-6D).

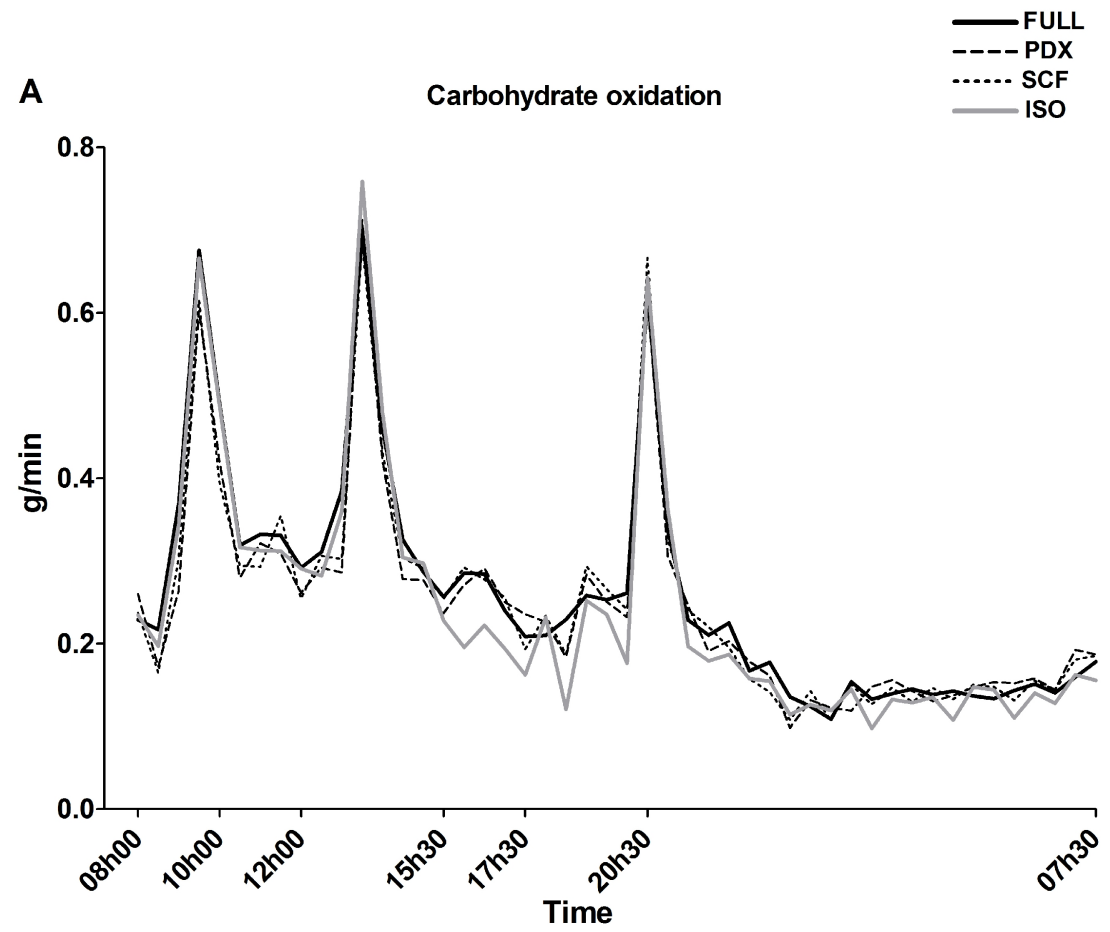

B

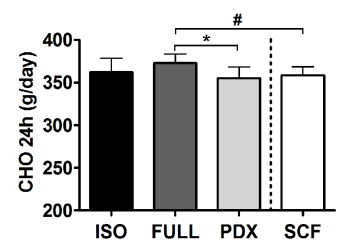

C

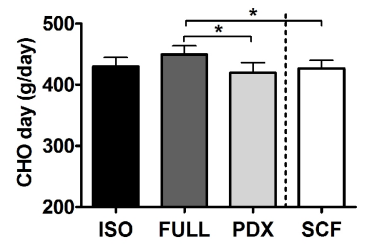

D

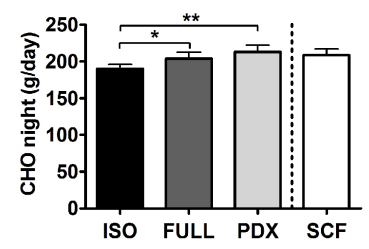

Figure 5. Carbohydrate oxidation (CHO) measured over 24 hours in a respiration chamber. (A) $\mathrm{CHO}$ measured over time. $\mathrm{CHO}$ measured during 24 hours (B), daytime (C), and night time (D). Repeated measures ANOVA were performed with LSD post-hoc testing. $n=16$. Data are mean \pm SEM. \# $p<0.1,{ }^{*} p<0.05,{ }^{* *} p<0.01$. 


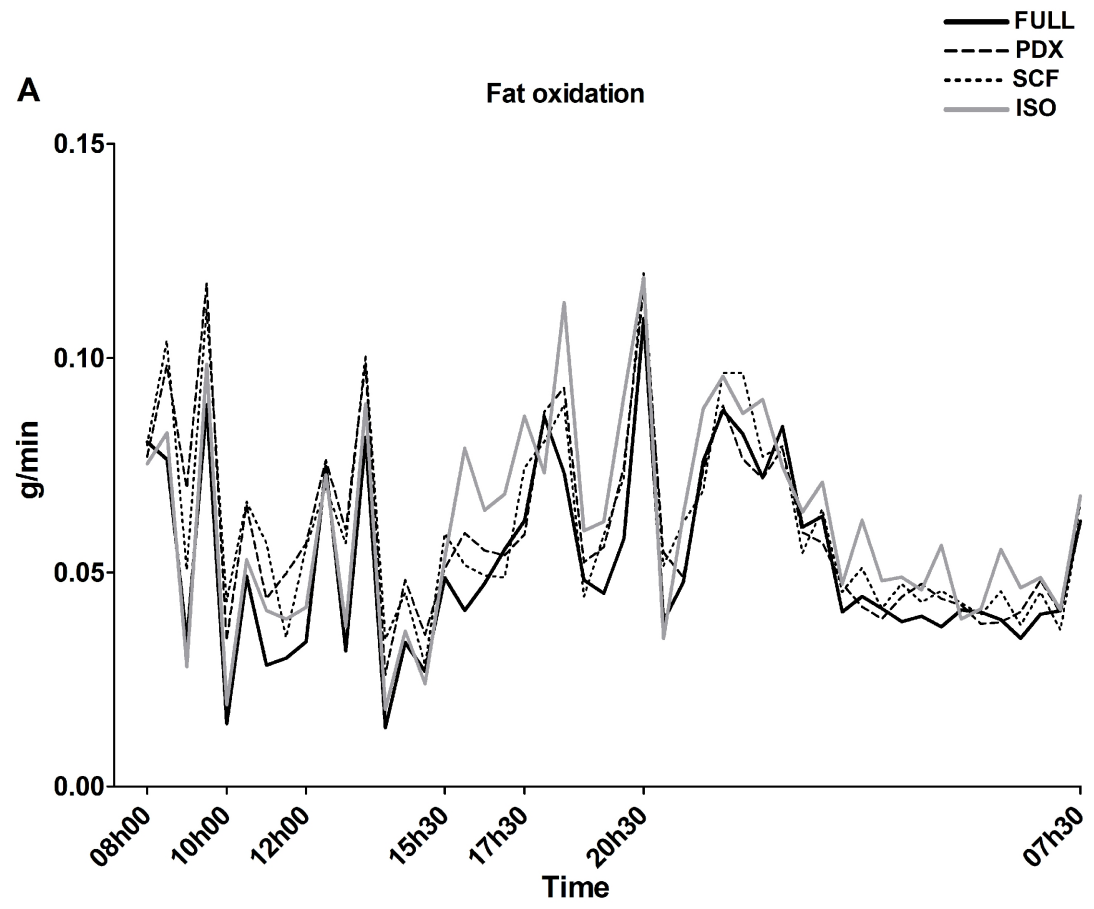

B

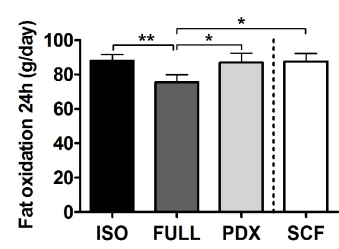

C

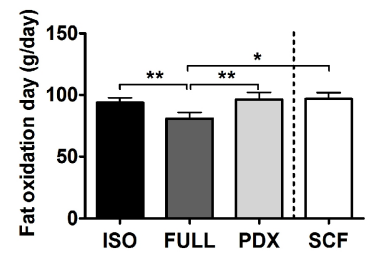

D

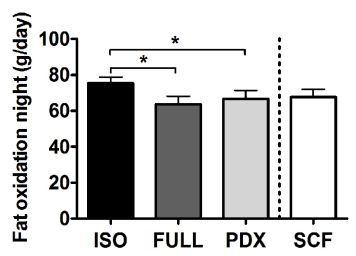

Figure 6. Fat oxidation measured over 24 hours in a respiration chamber. (A) Fat oxidation measured over time. Fat oxidation measured during 24 hours (B), daytime (C), and night time (D). Repeated measures ANOVA were performed with LSD post-hoc testing. $n=16$. Data are mean \pm SEM. * $p<0.05,{ }^{* *} p<0.01$.

\section{Hydrogen excretion}

The concentration hydrogen was significantly higher after the PDX diet over 24 hours compared with both control diets (Figure 7). The SCF diet also showed a higher breath hydrogen concentration compared with the full caloric diet. This indicates a higher colonic fermentation after both fiber diets. There was no difference between both fiber diets. The higher breath hydrogen response after the PDX and SCF diets decreased after dinner and showed the same response as the control diets. 


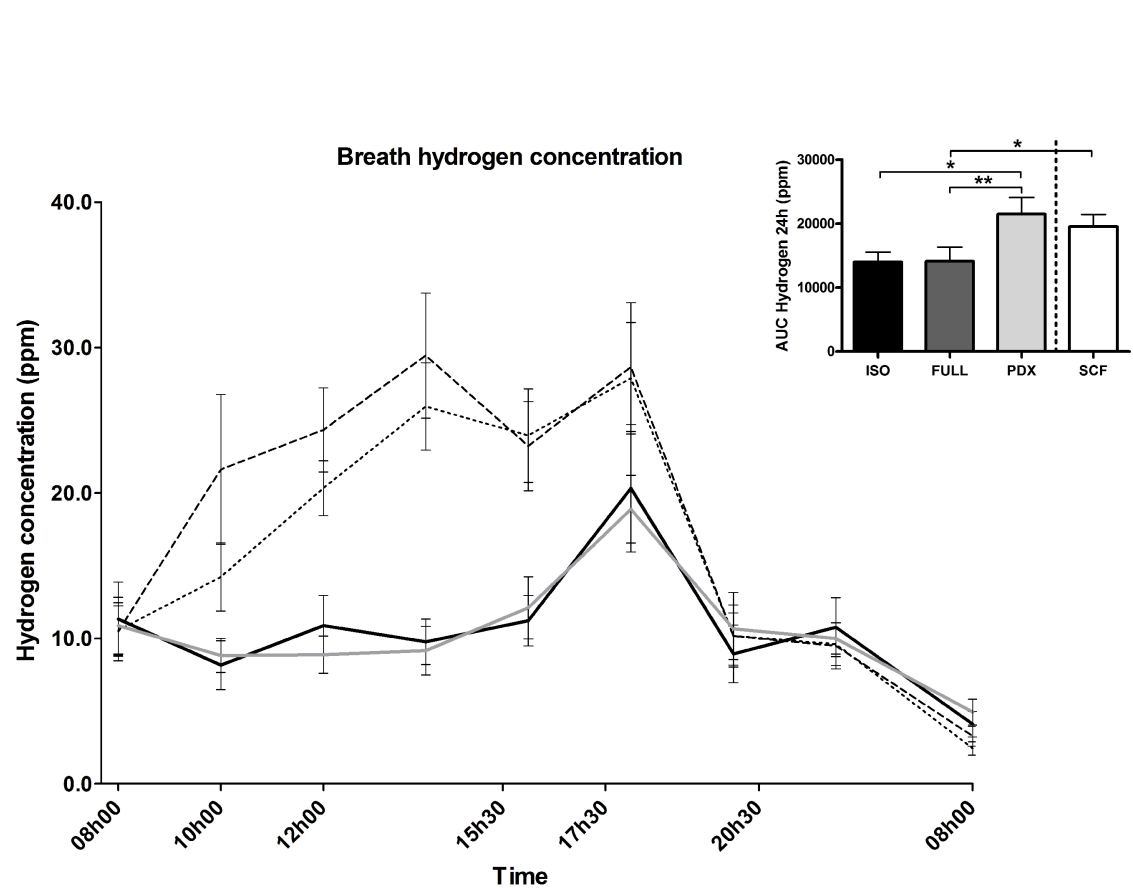

Figure 7. Breath hydrogen measurement over 24 hours. Upper right corner: AUC hydrogen over 24 hours. Repeated measures ANOVA were performed with LSD post-hoc testing using the integrated responses $(A \cup C) . n=18$. Data are mean \pm SEM. ${ }^{*} p<0.05,{ }^{* *} p<0.01$.

\section{VAS and well-being questionnaires}

Desire to eat and the feeling of hunger were significantly lower after the PDX diet compared with both control diets over 24 hours (Figure 8A and 8B). So, the reduced desire to eat and feeling of hunger was due to the effect of the PDX fibers per se. Feeling of fullness and satiety feelings were higher after the PDX diet compared with both control diets over 24 hours, but this effect was only observed in women (Figure 8C and $\mathbf{8 D}$ ). The effect on appetite was only seen with the PDX fibers since the SCF fibers showed a higher desire to eat $(p=0.09)$ and less satiety feelings ( $p=0.002$ ) compared with the PDX diet.

Subjects scored no feelings of nausea, burping, headache, vomiting and dizziness during all conditions. Only 1 male subject reported abdominal bloating in all conditions. The same subject reported stomach-intestinal cramps in all conditions except PDX. Diarrhea was scored present by 1 female in the PDX condition, 1 female in the SCF condition and 1 male in both fiber diets (Figure S1). The urge to defecate was the highest in the SCF condition with an average score of $1.9 \pm 0.3$ on a total score of 10 measured over 24 hours compared with $1.5 \pm 0.2$ in both the PDX and Full caloric condition (diet $p=0.02$, post-hoc Full vs. SCF $p=0.05$, PDX vs SCF $p=0.01$ ). 
$\mid$
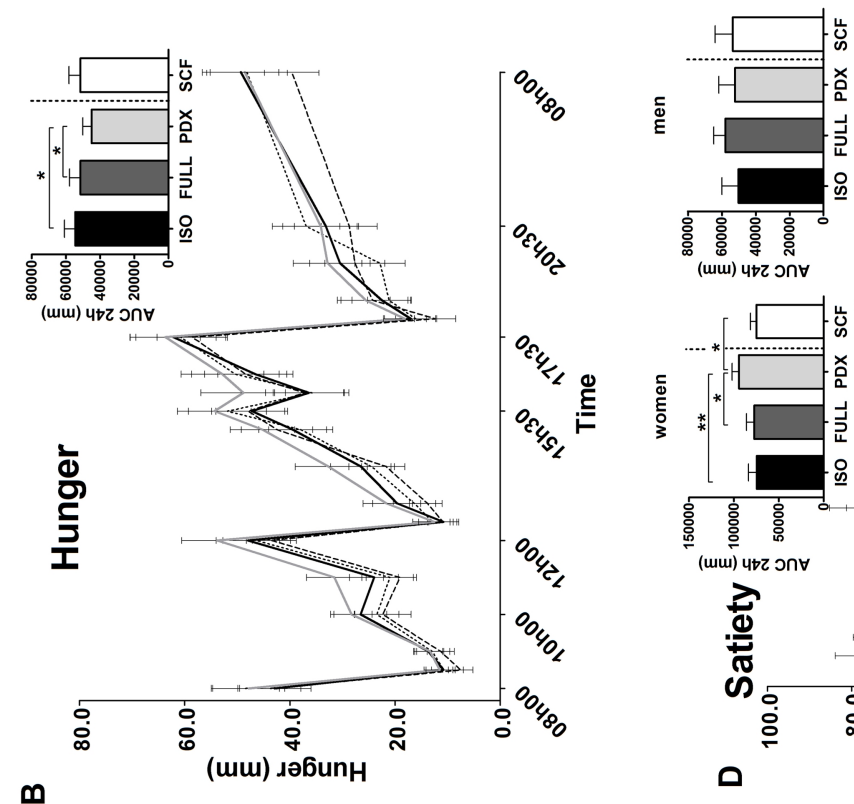

究 \&

के 응

원

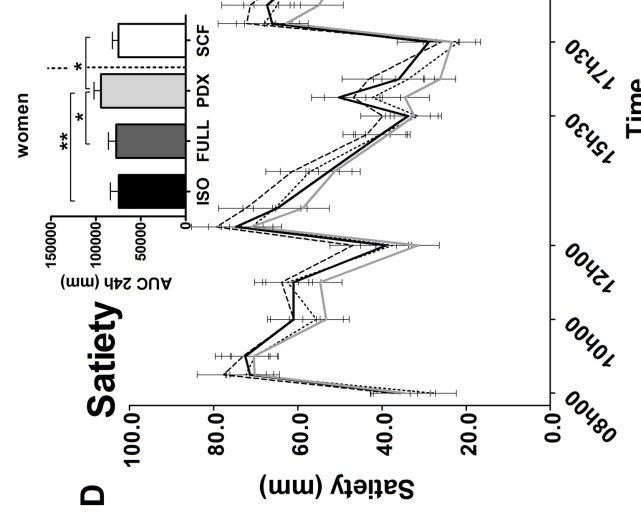

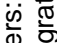

Ф

ठ․

등

은 인

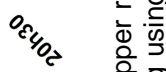

옹 옹

혼

ڤั

อ iุ

ब的

노

U)

बें ํํㅇ

고웜

웧

范

이

인

क⿺
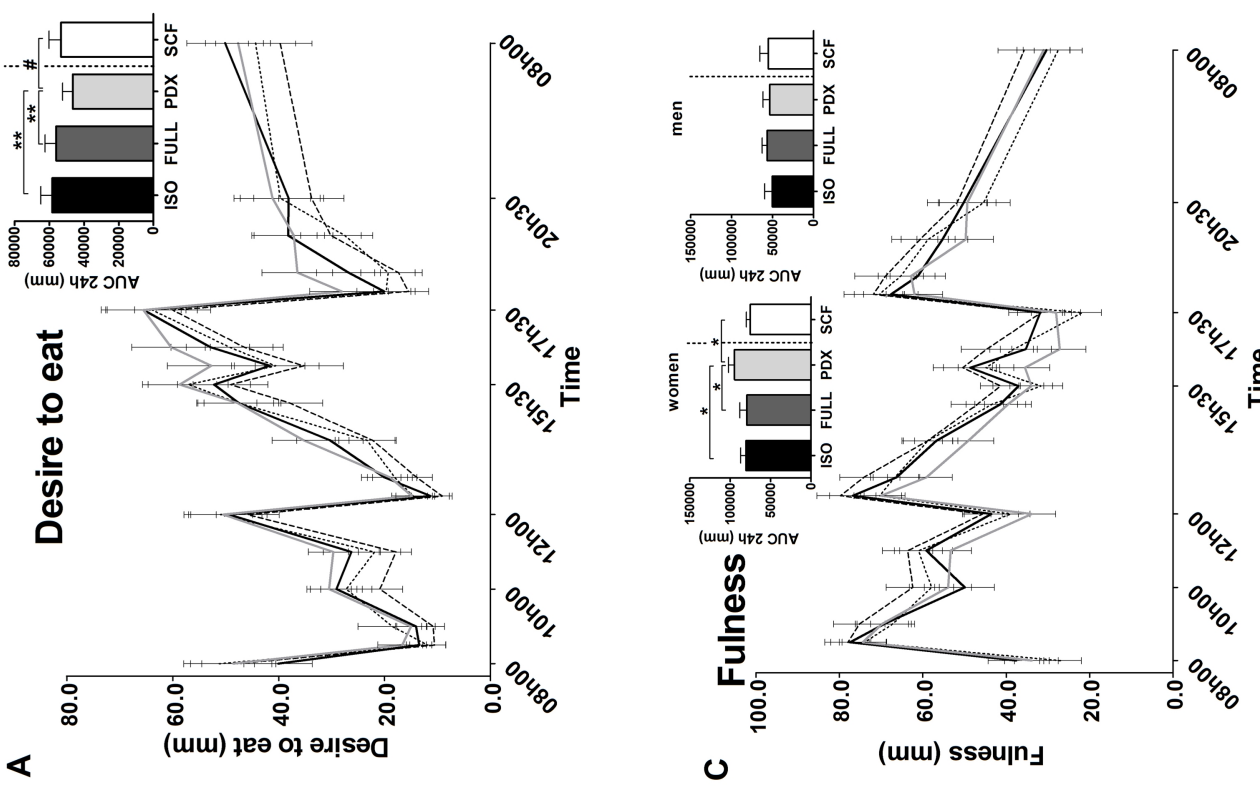

Фั ญे

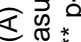

ம்

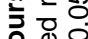

垔

స $\mathscr{O}^{*}$

屯亠

is $v$

紊

dis

胥 $\underset{\Phi}{\mathbb{E}} \sum_{\text {U }}$

$\Phi+1$

辛

क ष

을

证

$\Phi$

政西

응음

这突

$\infty$ 흐 는

亏气

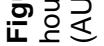




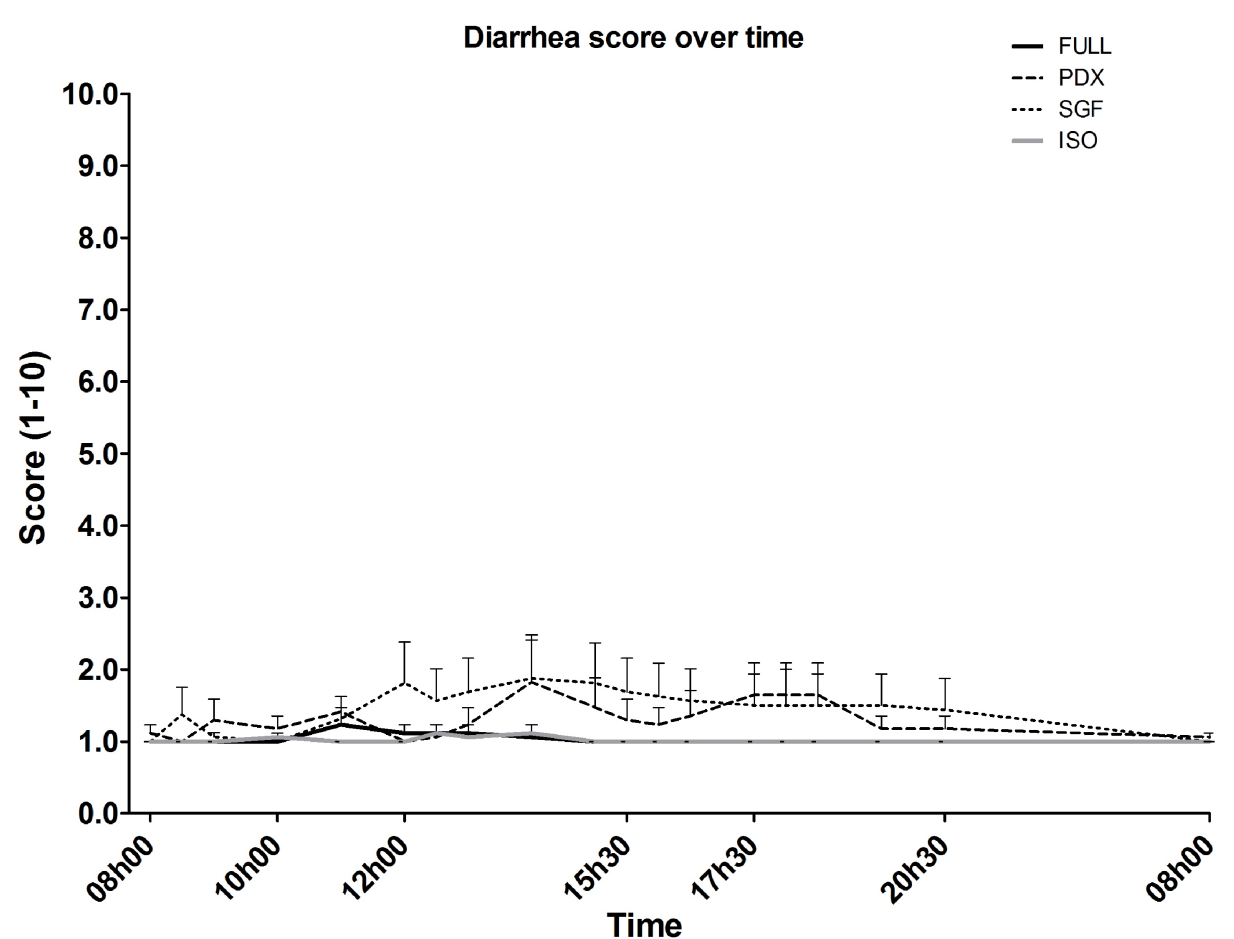

Figure $S 1$. Diarrhea score measured over time. $n=16$. Data are mean $\pm S E M$.

\section{Discussion}

Food components with health promoting properties have gained widespread interest. The present study shows that replacing available carbohydrates by the soluble fibers PDX and SCF reduced the peak glucose response, which was accompanied by a reduction in postprandial insulin responses. Furthermore, increased circulating FFA concentrations and concomitant a higher fat oxidation were observed. This was mainly attributed by the lower caloric value and the more negative energy balance of the high fiber diets and not the fibers per se. Nevertheless, PDX showed favorable effects on appetite, which appeared to be a specific characteristic of PDX independent of energy balance. The effects on appetite and fat oxidation can be regarded as a beneficial change in metabolic profile in relation to body weight control and insulin sensitivity if these effects persist over longer periods of time under ad libitum feeding conditions.

Replacing available carbohydrates by PDX or SCF reduced insulin responses in the postprandial state translating in a reduced insulin response over the whole day for SCF. However, no major effects on interstitial and plasma glucose responses were observed after both fiber diets, only the peak glucose responses after breakfast and lunch were lower. Several studies reported no effect of PDX on plasma glucose or insulin kinetics when PDX was given pure or as a drink with a 
mixed meal $(33,34)$. However, other studies reported a lower glucose response when PDX was incorporated in chocolate, sweetened dried cranberries and strawberry jam (35-37). Kendall et al. showed lower postprandial glucose and insulin responses for SCF when given as a lemonade drink (11). These studies investigated postprandial glucose and insulin concentrations after a single meal over a limited period of time rather than after three meals (breakfast and lunch contained PDX or SCF) during daytime in this study.

Furthermore, we investigated the effect of both fibers on substrate oxidation and energy expenditure. For the PDX and SCF diet, a higher fat oxidation and lower carbohydrate oxidation was observed compared with the full caloric diet. As indicated above, this increased fat oxidation was accompanied by reduced insulin responses (only significant for SCF over the whole day) and consequently higher FFA concentrations confirming our initial hypothesis that a fiber induced reduction in insulin concentrations may result in increased circulating FFA thereby increasing fat oxidation. Nevertheless, the increased fat oxidation was mainly attributed by the lower caloric value and not the fibers per se, since the iso-caloric control also showed a higher fat oxidation compared with the full caloric diet. Sparti et al. studied the effect of two different isoenergetic diets containing high or low unavailable carbohydrates on 24-hour substrate oxidation in lean subjects. However, total substrate oxidation did not differ between the two diets (38). In another study, substrate oxidation was measured for 5 hours after ingestion of meal containing resistant starch or rapidly digestible starch, with a difference of 103 $\mathrm{kJ}$ in energy content between the former and the latter meal. Resistant starch intake was followed by a lower glucose oxidation and a greater fat oxidation (39). In line with our results, the difference in energy content between both meals may have contributed to the increase in fat oxidation.

There were no differences in 24-hour energy expenditure between the diets. In previous studies in which the meal or diet was supplemented with dietary fibers, also no differences in 24-hour energy expenditure were reported (38, 40-42). DIT, $A E E$ and SMR were not different between the fiber and control diets. There is still a lot of controversy about the effect of dietary fibers on DIT. Some studies reported no effect of dietary fibers on DIT $(39,43)$, while others showed a reduced DIT $(44$, 45). The explanation for the discrepant results is not entirely clear but may relate to differences in the methodology for determining DIT, subject characteristics and different fiber characteristics.

Commensal bacteria ferment dietary fibers. The gases produced by fermentation are absorbed into the circulation and diffused into the pulmonary alveolus. Therefore, measurement of hydrogen in the breath reflects the anaerobic bacterial fermentation of dietary fiber in the colon $(14,46)$. In this study, we indeed showed that the concentration of hydrogen in the breath was significantly higher after the PDX and SCF diet compared with both control diets. This indicates a higher colonic 
fermentation after both fiber diets. There were no differences in hydrogen concentration between both fiber diets. Because colonic fermentation is an incomplete combustion, disregarding the fermentation process could lead to errors in estimating substrate oxidation from indirect calorimetry data $(47,48)$. However, in an experiment designed to quantify the measurement error in substrate oxidation due to the presence of fermentation, Poppitt et al. estimated the maximal error in carbohydrate oxidation after ingestion of $58 \mathrm{~g}$ unavailable carbohydrates to be $2 \%$. (49). Also, Heresbach et al. showed that when the additional excretion of breath hydrogen is $<20 \mathrm{ppm}$, the magniture of error in $\mathrm{VCO}_{2}$ measurement is small on average (47). Although it is important to measure hydrogen excretion in wholebody calorimetry studies in which fermentable carbohydrates are part of the diet, the effects on the calculation of energy expenditure and fuel selection in humans are small (40) and have had no impact on the conclusions of the present study.

Furthermore, the effect of both fibers on several measures of appetite were investigated. In our study, desire to eat and feeling of hunger were lowest after the PDX diet as compared to all other diets. Feeling of fullness and satiety feelings were higher after the PDX diet compared with both control diets, but this was only shown in women. Gender differences in satiety responses have been noted in previous studies, in which women reported higher post-meal satiety ratings and showed a reduced food intake $(50,51)$. The effect on appetite was not seen for SCF.

Peripheral fatty acid oxidation has long been implicated in the control of eating (52). Nevertheless, since the favourable effects on appetite were a specific characteristic of PDX while the increased fat oxidation was mainly determined by a negative energy balance, other mechanisms may be responsible for the PDXinduced reduction in appetite. Several factors may influence appetite like, the intrinsic physical properties of dietary fiber (bulking, gel formation and viscosity), modulation of gastric motor function, increased mastication, changes in gut hormones (ghrelin or GLP-1) and blunting of postprandial glucose and insulin responses (53-55). Additionally, it has been hypothesised that colonic fermentation resulting in increased production of SCFA could stimulate colonic L-cells to produce several appetite-regulating hormones such as PYY and GLP-1 (55). The responsible mechanisms remain to be elucidated.

In conclusion, replacement of available carbohydrates with PDX or SCF reduced the peak glucose response, reduced postprandial insulin responses and increased circulating FFA concentrations, which was accompanied by an increase in fat oxidation after both fiber diets. This effect was mainly attributed by the lower caloric value and not the fibers per se. Interestingly, PDX incorporated in food products may be of special interest since it has a suppressive effect on appetite control, which may have a significant effect on body weight control and improved metabolic health in the long term. 


\section{Acknowledgments}

We thank all subjects for participation in the present study. We gratefully thank Wendy Sluijsmans, Gabby Hul and Hasibe Aydeniz for their excellent analytical support. The authors would like to thank Tate and Lyle, Kaiseraugst, Villeneuve d'Ascq, France for providing us with the polydextrose and soluble corn fiber test products. The authors' responsibilities were as follows - EEB and EK designed research; EK conducted research; EK, JS and PFS analyzed data; EK performed statistical analysis; EK wrote the paper; EEB had primary responsibility for the final content; PFS and EEB reviewed/edited the manuscript. There were no conflicts of interest. This study was supported by Tate and Lyle, Kaiseraugst, Villeneuve d'Ascq, France. 


\section{References}

1. Obesity: preventing and managing the global epidemic. Report of a WHO consultation. World Health Organization technical report series 2000;894:i-xii, 1-253.

2. Diet, nutrition and the prevention of chronic diseases. World Health Organization technical report series 2003;916:i-viii, 1-149, backcover.

3. Slavin JL. Dietary fiber and body weight. Nutrition (Burbank, Los Angeles County, Calif 2005;21(3):411-8.

4. Anderson JW, Baird P, Davis RH, Jr., et al. Health benefits of dietary fiber. Nutrition reviews 2009;67(4):188-205.

5. King NA, Craig SA, Pepper T, Blundell JE. Evaluation of the independent and combined effects of xylitol and polydextrose consumed as a snack on hunger and energy intake over 10 d. The British journal of nutrition 2005;93(6):911-5.

6. Wanders AJ, van den Borne JJ, de Graaf C, et al. Effects of dietary fibre on subjective appetite, energy intake and body weight: a systematic review of randomized controlled trials. Obes Rev 2011;12(9):724-39.

7. Biorklund M, van Rees A, Mensink RP, Onning G. Changes in serum lipids and postprandial glucose and insulin concentrations after consumption of beverages with beta-glucans from oats or barley: a randomised dose-controlled trial. European journal of clinical nutrition 2005;59(11):1272-81.

8. Brennan CS. Dietary fibre, glycaemic response, and diabetes. Molecular nutrition \& food research 2005;49(6):560-70.

9. Juntunen KS, Niskanen LK, Liukkonen KH, Poutanen KS, Holst JJ, Mykkanen HM. Postprandial glucose, insulin, and incretin responses to grain products in healthy subjects. The American journal of clinical nutrition 2002;75(2):254-62.

10. Ulmius $M$, Johansson $A$, Onning $G$. The influence of dietary fibre source and gender on the postprandial glucose and lipid response in healthy subjects. European journal of nutrition 2009;48(7):395-402.

11. Kendall CW, Esfahani A, Hoffman AJ, et al. Effect of novel maize-based dietary fibers on postprandial glycemia and insulinemia. Journal of the American College of Nutrition 2008;27(6):711-8.

12. Saris WH. Sugars, energy metabolism, and body weight control. The American journal of clinical nutrition 2003;78(4):850S-7S.

13. Brand-Miller J, McMillan-Price J, Steinbeck K, Caterson I. Dietary glycemic index: health implications. Journal of the American College of Nutrition 2009;28 Suppl:446S-9S.

14. Chinda D, Nakaji S, Fukuda S, et al. The fermentation of different dietary fibers is associated with fecal clostridia levels in men. The Journal of nutrition 2004;134(8):1881-6.

15. Flood MT, Auerbach MH, Craig SA. A review of the clinical toleration studies of polydextrose in food. Food Chem Toxicol 2004;42(9):1531-42.

16. Sanders L KC, Maki K, Stewart M, Slavin J, Potter S. A novel maize-based dietary fiber is well tolerated in humans. FASEB J 2008;22:Ib761.

17. Bruhwyler J, Carreer F, Demanet E, Jacobs H. Digestive tolerance of inulin-type fructans: a double-blind, placebo-controlled, cross-over, dose-ranging, randomized study in healthy volunteers. International journal of food sciences and nutrition 2008:1-11.

18. Raninen K, Lappi J, Mykkanen H, Poutanen K. Dietary fiber type reflects physiological functionality: comparison of grain fiber, inulin, and polydextrose. Nutrition reviews $2011 ; 69(1): 9-21$.

19. Charalampopoulos D, Rastall RA, SpringerLink (Online service). Internet: http://dx.doi.org/10.1007/978-0-387-79058-9

20. Vester Boler BM, Rossoni Serao MC, Bauer LL, et al. Digestive physiological outcomes related to polydextrose and soluble maize fibre consumption by healthy adult men. The British journal of nutrition 2011:1-8.

21. Ellis AC, Hyatt TC, Hunter GR, Gower BA. Respiratory quotient predicts fat mass gain in premenopausal women. Obesity (Silver Spring, Md;18(12):2255-9.

22. Bornet FR, Jardy-Gennetier AE, Jacquet N, Stowell J. Glycaemic response to foods: impact on satiety and long-term weight regulation. Appetite 2007;49(3):535-53.

23. Langhans W. Fatty acid oxidation in the energostatic control of eating--a new idea. Appetite 2008;51(3):446-51. 
24. Ravussin E, Smith SR. Increased fat intake, impaired fat oxidation, and failure of fat cell proliferation result in ectopic fat storage, insulin resistance, and type 2 diabetes mellitus. Annals of the New York Academy of Sciences 2002;967:363-78.

25. Holloway GP, Bonen A, Spriet LL. Regulation of skeletal muscle mitochondrial fatty acid metabolism in lean and obese individuals. The American journal of clinical nutrition 2009;89(1):455S-62S.

26. Schoffelen PF, Westerterp KR, Saris WH, Ten Hoor F. A dual-respiration chamber system with automated calibration. J Appl Physiol 1997;83(6):2064-72.

27. Schrauwen P, van Marken Lichtenbelt WD, Westerterp KR. Energy balance in a respiration chamber: individual adjustment of energy intake to energy expenditure. Int $\mathrm{J}$ Obes Relat Metab Disord 1997;21(9):769-74.

28. Westerterp-Plantenga MS, van Marken Lichtenbelt WD, Strobbe H, Schrauwen P. Energy metabolism in humans at a lowered ambient temperature. European journal of clinical nutrition 2002;56(4):288-96.

29. Frayn KN. Calculation of substrate oxidation rates in vivo from gaseous exchange. J Appl Physiol 1983;55(2):628-34.

30. Weir JB. New methods for calculating metabolic rate with special reference to protein metabolism. The Journal of physiology 1949;109(1-2):1-9.

31. Westerterp KR, Wilson SA, Rolland V. Diet induced thermogenesis measured over $24 \mathrm{~h}$ in a respiration chamber: effect of diet composition. Int $\mathrm{J}$ Obes Relat Metab Disord 1999;23(3):287-92.

32. Flint A, Raben A, Blundell JE, Astrup A. Reproducibility, power and validity of visual analogue scales in assessment of appetite sensations in single test meal studies. Int $\mathrm{J}$ Obes Relat Metab Disord 2000;24(1):38-48.

33. Craig SAS, Holden JF, Troup JP, Auerbach MH, Frier HI. Polydextrose as soluble fiber: physiological and analytical aspects. Cereal Foods World 1998;43:370-6.

34. Schwab U, Louheranta A, Torronen A, Uusitupa M. Impact of sugar beet pectin and polydextrose on fasting and postprandial glycemia and fasting concentrations of serum total and lipoprotein lipids in middle-aged subjects with abnormal glucose metabolism. European journal of clinical nutrition 2006;60(9):1073-80.

35. Shimomura $\mathrm{Y}$, Maeda K, Nagasaki M, et al. Attenuated response of the serum triglyceride concentration to ingestion of a chocolate containing polydextrose and lactitol in place of sugar. Biosci Biotechnol Biochem 2005;69(10):1819-23.

36. Kurotobi T, Fukuhara K, Inage H, Kimura S. Glycemic index and postprandial blood glucose response to Japanese strawberry jam in normal adults. J Nutr Sci Vitaminol (Tokyo) 2010;56(3):198-202.

37. Wilson T, Luebke JL, Morcomb EF, et al. Glycemic responses to sweetened dried and raw cranberries in humans with type 2 diabetes. J Food Sci 2010;75(8):H218-23.

38. Sparti A, Milon H, Di Vetta V, et al. Effect of diets high or low in unavailable and slowly digestible carbohydrates on the pattern of 24-h substrate oxidation and feelings of hunger in humans. The American journal of clinical nutrition 2000;72(6):1461-8.

39. Tagliabue A, Raben A, Heijnen ML, Deurenberg P, Pasquali E, Astrup A. The effect of raw potato starch on energy expenditure and substrate oxidation. The American journal of clinical nutrition 1995;61(5):1070-5.

40. Poppitt SD, Livesey G, Elia M. Energy expenditure and net substrate utilization in men ingesting usual and high amounts of nonstarch polysaccharide. The American journal of clinical nutrition 1998;68(4):820-6.

41. Brown JC, Livesey G. Energy balance and expenditure while consuming guar gum at various fat intakes and ambient temperatures. The American journal of clinical nutrition 1994;60(6):956-64.

42. Ryttig KR, Lammert O, Nielsen E, Garby L, Poulsen K. The effect of a soluble dietary fibre supplement on 24-hour energy expenditure during a standardized physical activity programme. International journal of obesity 1990;14(5):451-5.

43. Ritz P, Krempf M, Cloarec D, Champ M, Charbonnel B. Comparative continuous-indirectcalorimetry study of two carbohydrates with different glycemic indices. The American journal of clinical nutrition 1991;54(5):855-9.

44. Scalfi $L$, Coltorti A, D'Arrigo E, et al. Effect of dietary fibre on postprandial thermogenesis. International journal of obesity 1987;11 Suppl 1:95-9.

45. Raben A, Christensen NJ, Madsen J, Holst JJ, Astrup A. Decreased postprandial thermogenesis and fat oxidation but increased fullness after a high-fiber meal compared with a low-fiber meal. The American journal of clinical nutrition 1994;59(6):1386-94. 
46. Le Marchand L, Wilkens LR, Harwood P, Cooney RV. Use of breath hydrogen and methane as markers of colonic fermentation in epidemiologic studies: circadian patterns of excretion. Environmental Health Perspectives 1992;98:199-202.

47. Heresbach D, Flourie B, Briet F, Achour L, Rambaud JC, Messing B. Effect of colonic fermentation on respiratory gas exchanges measured in the postabsorptive state. The American journal of clinical nutrition 1995;62(5):973-8.

48. Ritz $P$, Cloarec $D$, Beylot $M$, et al. Effects of colonic fermentation on respiratory gas exchanges following a glucose load in man. Metabolism: clinical and experimental 1993;42(3):347-52.

49. Poppitt SD, Livesey G, Faulks RM, Roe M, Prentice AM, Elia M. Circadian patterns of total 24-h hydrogen and methane excretion in humans ingesting nonstarch polysaccharide (NSP) diets and the implications for indirect calorimetric and D2 180 methodologies. European journal of clinical nutrition 1996;50(8):524-34.

50. Cornier MA, Salzberg AK, Endly DC, Bessesen DH, Tregellas JR. Sex-based differences in the behavioral and neuronal responses to food. Physiology \& behavior 2010;99(4):538-43.

51. Hess JR, Birkett AM, Thomas W, Slavin JL. Effects of short-chain fructooligosaccharides on satiety responses in healthy men and women. Appetite 2011;56(1):128-34.

52. Scharrer E, Langhans W. Control of food intake by fatty acid oxidation. Am J Physiol 1986;250(6 Pt 2):R1003-6.

53. Papathanasopoulos A, Camilleri M. Dietary fiber supplements: effects in obesity and metabolic syndrome and relationship to gastrointestinal functions. Gastroenterology 2010;138(1):65-72 e1-2.

54. Willis HJ, Eldridge AL, Beiseigel J, Thomas W, Slavin JL. Greater satiety response with resistant starch and corn bran in human subjects. Nutrition research (New York, NY 2009;29(2):100-5.

55. Kristensen $M$, Jensen MG. Dietary fibres in the regulation of appetite and food intake. Importance of viscosity. Appetite 2011;56(1):65-70. 


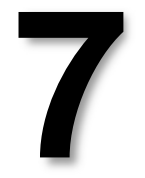

General Discussion 


\section{Obesity and insulin resistance}

Obesity is increasing in epidemic proportions throughout the whole world (1). This alarming increase in obesity is associated with a lower age of onset and an increased incidence and prevalence of obesity-related comorbidities like type 2 diabetes mellitus (T2DM) (2). Changes in lifestyle are considered to play an important role in the etiology of obesity and T2DM. Manipulation of diet and physical activity are the first-choice treatment for these metabolic diseases (3). Lifestyle intervention programs combining dietary advice and an increased physical activity have been shown to be effective in the prevention of $\operatorname{T2DM}(4,5)$. However, more than $30 \%$ of the high risk subjects do not respond to the intervention indicating the need for additional preventive strategies. Metabolic inflexibility, defined as an impaired capacity to increase fat oxidation in the fasted state and to suppress fat oxidation after a meal may contribute to the accumulation of lipids and lipid metabolites within the skeletal muscle (6). The accumulation, composition and localization of bioactive lipid metabolites may interfere with insulin signaling, thereby promoting the development of insulin resistance and $\operatorname{T2DM}(7,8)$. Identification of foods, which could influence specific physiological targets to improve metabolic flexibility may be helpful in the prevention and management of overweight and obesity as well as insulin resistance. Therefore, in this thesis we investigated the effects of several dietary interventions on body weight control as well as the prevention and/or reversal of insulin resistance by modulation of fat metabolism.

\section{Fatty acid handling in the prediabetic state}

The underlying metabolic and molecular mechanisms responsible for the impairment in metabolic flexibility in the insulin resistant or prediabetic state are not completely understood (9). It has been suggested that the metabolic profile of the insulin resistant muscle is more directed towards fat storage than towards oxidation $(9,10)$.

It has been shown that T2DM subjects have a significantly elevated ACC-2 mRNA expression in skeletal muscle (11). This increase in ACC-2 may promote malonylCoA formation which in turn leads to inhibition of CPT-1, a lower transport of FFA into the mitochondria and a decreased fat oxidation (12). Also a set of genes involved in oxidative phosphorylation including PGC1 $\alpha$, has been shown to be coordinately downregulated in the skeletal muscle of T2DM patients $(13,14)$. In chapter 2 we investigated whether the prediabetic state is accompanied by changes in the expression of genes involved in fatty acid handling during fasting and insulin-mediated conditions. We observed no differences in expression of 
genes involved in lipogenesis and lipid oxidation between the impaired glucose tolerant (IGT) and normal glucose tolerant (NGT) state. This may be explained by the fact that differences in the degree of insulin resistance between the subject groups were less extreme than in previous studies $(11,13-15)$. After the hyperinsulinemic clamp, several genes were upregulated, including $P G C 1 \alpha$, SREBP-1C, SREBP-2 and ACC-2. Interestingly, the clamp-induced increase in SREBP-2 gene expression was more pronounced in IGT men as compared to NGT men. This may reflect an increased activation of the novo membrane synthesis to repair the mitochondrial membrane damage possibly induced by an increased lipid peroxidation in the prediabetic state (16). However, further research is necessary to elucidate the exact role of SREBP-2 in skeletal muscle.

\section{Calorie restriction}

Calorie restriction has consistently been proven to produce weight loss and to have beneficial health effects like an improved insulin sensitivity $(17,18)$, possibly mediated via modulation of fat metabolism. Previous studies have reported that weight loss showed either an improvement (19) or no change (20) in fasting fat oxidation. After weight loss, obese prediabetic subjects have been shown to improve their capacity to switch from fat oxidation to carbohydrate oxidation after a meal (19). Altogether, there are clear indications that weight loss is able to partly reverse postprandial impairments in substrate oxidation, whereas with respect to the fasting condition, improved regulation of fat oxidation could not be confirmed in all studies. To elucidate the effect of weight loss on fasting skeletal muscle fat metabolism, we investigated in chapter 2 the impact of weight loss on fatty acid metabolism-related gene expression in obese IGT subjects. After the 12 week VLCD, the obese IGT man lost approximately $15 \mathrm{~kg}$ of body weight, which was accompanied by an increase of $71 \%$ in insulin sensitivity and a tendency towards an increase in muscle oxidative capacity as evidenced by CS activity. Nevertheless, no changes in skeletal muscle gene expression of several target genes (PGC1 $\alpha$, PPAR $\alpha$, ACC-2, SREBPs and ChREBP) were observed. This is consistent with Simoneau et al. (9) who also did not detect changes in several different markers of skeletal muscle FA metabolism in obese NGT men after a weight loss intervention based on a VLCD, but is not consistent with all other studies showing an upregulation of genes involved in lipid oxidation (21-23). Our results may imply that changes in the postprandial state possibly play a larger role in lipid-induced insulin resistance. However, gene expression changes do not necessarily translate into functional proteins so it could be that some key steps in lipid metabolism are involved in the improvement in insulin sensitivity, in particular since we observed a tendency towards an increased oxidative capacity. 
Furthermore, we cannot exclude that small differences in gene expression after weight loss may have been missed because of the relatively small sample size, and the results need confirmation in future studies.

\section{Resveratrol: a potential calorie restriction mimetic?}

Several beneficial effects of resveratrol have been described including antiinflammatory, antioxidant, cardio-protective and anti-aging actions (24). Although originally the focus was mainly directed towards anti-oxidant properties of resveratrol (25), more recently cellular effects on fatty acid metabolism and mitochondrial function have been described. Resveratrol has been shown to exert favourable effects in rodents fed a high-calorie diet. The animals were less prone to develop insulin resistance, which was related to the induction of genes for oxidative phosphorylation and increased mitochondrial biogenesis (26). By reversing disturbances in fat metabolism resveratrol may be able to improve the success of lifestyle interventions with respect to insulin sensitivity and body weight control. In chapter 3, we investigated the effect of 30 days resveratrol supplementation on energy metabolism and metabolic profile in 11 healthy obese men. The hypothesis was that an improvement in mitochondrial function together with an increased mitochondrial biogenesis improves skeletal muscle metabolic flexibility and (fat) oxidative capacity. This may reduce bioactive lipid metabolite accumulation and may improve insulin sensitivity. Our microarray data clearly suggested strong involvement of mitochondrial function in the beneficial effects of resveratrol. Therefore, we performed more detailed analyses of mitochondrial function which revealed that resveratrol had beneficial effects on mitochondrial respiration when octanoyl-carnitine was used as a substrate, but not when only glutamate was used. These data suggest that resveratrol specifically improves muscle fat oxidative mitochondrial capacity. The underlying molecular mechanisms contributing to the improvement in mitochondrial activity may be mediated by AMPK which was activated by resveratrol. This resulted in increased protein content of the AMPK downstream effector SIRT1 as well as the mitochondrial master regulator PGC1 $\alpha$, accompanied by increased citrate synthase activity. The fact that mtDNA copy number, OXPHOS protein content, and $\mathrm{PCr}$ recovery were not changed in resveratrol treated subjects suggests that 30 days resveratrol treatment mostly affects mitochondrial efficiency, not abundance. It is possible that more long-term treatment would cause these parameters to change as well.

Furthermore, we investigated whether this improvement in mitochondrial efficiency would be reflected on whole body level. Over 24 hours we observed a higher RQ in the resveratrol group especially apparent after periods of feeding suggesting an improved metabolic flexibility. This was confirmed in the postprandial test were we 
showed an improvement in insulin mediated suppression of postprandial adipose tissue lipolysis and a more pronounced reduction in whole body fat oxidation after resveratrol supplementation as compared to placebo. This suggests an improved switch in substrate oxidation after a meal.

With respect to energy metabolism, subjects showed a lower sleeping metabolic rate after resveratrol supplementation, without changing 24 hour energy expenditure. It should be noted that sleeping metabolic rate is the component of human energy metabolism that is most sensitive to metabolic changes-as it is not affected by physical activity-and small differences in sleeping energy expenditure can be detected with high accuracy. In line, we observed that basal and postprandial energy expenditure were also lower after 30 days of resveratrol supplementation. The $2 \%-4 \%$ reduction in energy expenditure upon resveratrol treatment may reflect an increased metabolic efficiency and is consistent with the effects observed after calorie restriction (6\% reduction) $(27,28)$. Long-term supplementation of resveratrol may thus contribute to weight gain if energy intake is not adjusted to the reduction in energy expenditure. An approach to overcome this lower energy expenditure is by supplementing resveratrol together with other food components that can stimulate energy expenditure like caffeine, capsaicin or green tea extract $(29,30)$. However, this has to be further investigated in long-term human studies.

Since our data showed that resveratrol clearly induced a shift in mitochondrial substrate selection and an improvement in metabolic flexibility, we investigated whether this was also reflected in liver and skeletal muscle lipid accumulation. Intrahepatic lipid content was lower after 30 days of resveratrol supplementation compared with placebo. This was paralleled by lower plasma ALAT values, both indicating improved liver function. In contrast, intramyocellular lipid (IMCL) content was markedly increased, but since this was concomitant with an improvement in muscle fat oxidative capacity, this may hint towards endurance training-like effects of resveratrol $(31,32)$. Endurance athletes have elevated IMCL levels but are extremely insulin sensitive because of their high muscle oxidative capacity (33).

Furthermore, insulin sensitivity was also improved after resveratrol as evidenced by a lower HOMA-index. Several human intervention studies reported the insulinsensitizing effect of resveratrol. Crandall et al. treated 10 IGT patients for 4 weeks with resveratrol and observed a reduction in postprandial glucose levels without an increase in insulin production (34). In T2DM patients, 4-weeks resveratrol supplementation improved insulin sensitivity based on HOMA-IR and delayed the glucose peak following a standardized meal (35). However, Ghanim et al. did not show an effect on fasting glucose, insulin and HOMA-IR scores following 6 weeks resveratrol supplementation (in $\mathrm{P}$. cuspidatum extract) in healthy individuals (36). From these interventions it can be concluded that subjects with IGT or T2DM may be most susceptible to resveratrol supplementation and may therefore benefit the 
most from this intervention.

Pronounced effects of resveratrol have also been reported on lipolysis, adipogenesis and inflammation in isolated adipocytes and on adipose tissue of murine animals (37). These effects may possibly modulate adipose tissue function and contribute to a reduced body fat accumulation and improved insulin sensitivity $(26,38)$. However, data on the effects of resveratrol on adipose tissue function in the human in vivo situation are lacking. Therefore, in chapter 4 the effect of resveratrol on adipose tissue morphology and the underlying mechanisms involved were investigated using microarray analysis. Resveratrol significantly decreased adipocyte size, with a shift towards a lower proportion of large and very large adipocytes and an increased proportion of small adipocytes. This reduced mean adipocyte size may underlie the improved insulin sensitivity as reported in chapter 3, since adipocyte size is an independent determinant of insulin sensitivity in humans (39). Microarray analysis revealed downregulation of pathways like Wnt, Notch and BMP/TGF- $\beta$ signaling and upregulation of gene sets contributing to cell cycle, which suggest an enhanced adipogenesis after resveratrol supplementation. This increased adipogenesis could possibly explain the reduced adipocyte size after resveratrol. Furthermore, GSEA revealed an increase in gene sets associated with activation of a lysosomal pathway of lipid breakdown (autophagy). Until recently, mobilization of lipids by the classic lipolytic pathway has only been attributed to lipid droplet associated proteins and lipases. The involvement of autophagy in lipid degradation was first reported by Singh et al. (40) who demonstrated that clearance of lipid droplets is mediated by lipid specific autophagy in hepatocytes and liver. Major support for the involvement of autophagy in our study was also the activation of the transcription factor EB (TFEB), which has been shown to control multiple steps of the autophagic pathway and to ensure prolonged and sustained activation of autophagy (41). The finding that the lysosomal degradative pathway is a critical regulator of hepatocyte lipid metabolism opens the possibility that it may function in a similar way in adipose tissue. Studies to date have not determined the effect of autophagy on differentiated adipocytes. In preadipocytes, it has been shown that inhibition of autophagy may block adipogenesis (42). Therefore, in the present study, the lysosome and phagosome pathways, may have contributed both to an induction of adipogenesis as well as to alternative lipid breakdown. These data suggest that autophagy may serve as an important target for the treatment of metabolic diseases like obesity and T2DM and that resveratrol may be an interesting therapeutic tool to induce this process but this needs to be further elucidated.

Furthermore in chapter 4, several gene sets involved in the immune response were upregulated in adipose tissue after resveratrol treatment. This may reflect an increase in cellular stress induced by the reduction in adipocyte size caused by traction forces between the adipocyte and the surrounding extracellular matrix (43). 
This in turn may induce upregulation of inflammatory genes and pro-inflammatory adipokine production, as shown after weight loss $(44,45)$. On the other hand, upregulation of immune response pathways may be a physiological response to a higher lipid turnover (i.e. lysosomal lipid breakdown). As reported in chapter 3, we also observed a decrease in circulating plasma II- 6 and TNF- $\alpha$ concentrations and a downregulation of gene sets associated with immune response in skeletal muscle in these subjects following resveratrol supplementation. These findings indicate that the increased immune response in adipose tissue could represent a local rather than a systemic response. Further studies in humans are necessary to investigate the tissue specific effects of resveratrol supplementation on inflammation.

In summary, 30 days resveratrol supplementation clearly improved muscle fat oxidative mitochondrial capacity and metabolic flexibility. Moreover, resveratrol induced a shift towards an increased proportion of metabolic favorable small adipocytes. This phenotype was accompanied by a gene expression profile indicative of increased adipogenesis and an alternative pathway of lipid breakdown which may contribute to an increased lipid buffering capacity of the adipose tissue. This may reduce the supply of lipids to non-adipose tissues and together with the increased oxidative capacity may contribute to a reduction in ectopic lipid accumulation as was observed in the liver. The reduced intrahepatic lipid content together with an efficient lipid turnover in skeletal muscle may have contributed to the improvement in insulin sensitivity. From this we can conclude that resveratrol has considerable potential to improve health and prevent chronic disease in humans. However, it is not yet certain if long-term resveratrol supplementation will maintain these physiological benefits.

\section{Dietary fatty acids}

An overconsumption of saturated fats has been implicated as a causative factor for the development of insulin resistance, whereas certain monounsaturated fats and PUFAs and, particularly n-6 fatty acids, appear to have no adverse effects or even fairly positive effects on the action of insulin (46). However, the underlying mechanisms how dietary fatty acids may affect insulin sensitivity and how the risk or severity of insulin resistance can be modulated by fat quantity and quality are currently under debate. A change in dietary fat quality (an increase in unsaturated fatty acids) may differentially modulate circulating lipids, skeletal muscle fatty acid handling and fuel partitioning within the muscle towards less accumulation of lipid metabolites and increased oxidative capacity which may contribute to an improved insulin sensitivity. Therefore, in chapter $\mathbf{5}$, the objective was to examine the acute 
effects of meals with various fatty acid compositions on skeletal muscle FA processing and postprandial insulin sensitivity in obese, insulin-resistant men. The meals consisted of SFA (35.5 En\%), MUFA (42.2 En\%) or PUFA (34.8 En\%). The PUFA meal contained $n-6$ safflower oil and $n-3$ fish oil with a ratio of $1: 1$. Ingestion of the PUFA meal resulted in an improved postprandial insulin sensitivity compared with SFA. Our data are in line with those of previous studies, showing that a PUFA meal improved insulin sensitivity as compared with an SFA meal $(47,48)$. Furthermore, we found that the effect of MUFA was intermediate between the SFA meal and the PUFA meal, which agrees with studies that have shown beneficial effects of MUFA on insulin sensitivity as compared with SFA (49-51). Moreover, underlying mechanisms for the improvement in insulin sensitivity after the PUFA meal were investigated by using a dual stable isotope technique. With this technique, the contribution of dietary fat (CM-TAG) vs. endogenous fat (FFA and VLDL-TAG) to in vivo skeletal muscle fatty acid metabolism could be investigated by using two different labels. $\left[\mathrm{U}-{ }^{13} \mathrm{C}\right]$-palmitate was added to a high fat mixed meal to label CM-TAG in the circulation and $\left[{ }^{2} \mathrm{H}_{2}\right]$-palmitate was infused intravenously to label the endogenous pool of FA. As $\left[{ }^{2} \mathrm{H}_{2}\right]$-palmitate can be incorporated into TAG in the liver, $\left[{ }^{2} \mathrm{H}_{2}\right]$-labelled TAG represents VLDL-TAG in the circulation. Moreover, this stable isotope technique was combined with measurements of arterio-venous concentration differences across the forearm muscle to investigate skeletal muscle FA handling and the specific contribution of the different fat sources.

We observed a reduced uptake of TAG-derived FA by the forearm muscle in the postprandial period after ingestion of a meal high in PUFA as compared with the SFA meal. Furthermore, we showed a higher incorporation of dietary $\left[\mathrm{U}-{ }^{13} \mathrm{C}\right]-$ palmitate from the muscle FFA pool in the different lipid fractions (higher fractional synthetic rate [FSR]) in skeletal muscle with unsaturated fatty acids compared with saturated fatty acids, indicating an increased muscle lipid turnover. Moreover, microarray analysis of skeletal muscle revealed a lesser downregulation of the mitochondrial oxidative genes after the PUFA meal than after the SFA or MUFA meal. Together, these data indicate that a PUFA-induced improvement in postprandial insulin sensitivity may be accompanied by a lower lipid uptake and possibly less accumulation of fat in the muscle and a higher intramuscular lipid turnover as compared to SFA. This is consistent with the findings of a recent study, which showed that insulin resistance is associated with a reduced lipid turnover as evidenced by a lower FSR of intramuscular TAG (52). Nevertheless, we cannot exclude that the increase in insulin sensitivity after the PUFA meal may also be explained by other mechanisms. For example, differences in the secretion of gut hormones (glucagon-like peptide 1 and cholecystokinin) could potentiate differential insulin secretion and insulin sensitivity according to the fatty acid composition of the meals $(51,53)$. Thus, the exact mechanisms responsible for the differential postprandial insulin sensitivity should be further elucidated in future 
studies. Furthermore, it remains to be established whether these acute effects may contribute to findings after long-term dietary intervention. Three large long-term intervention studies, KANWU study, LIPGENE study and the RISCK trial, did not find major effects on insulin resistance when SFA were replaced by an equal amount of MUFA (54-56). However, substitution of SFA with MUFA did improve insulin sensitivity in the KANWU study when total fat intake was below $38 \mathrm{En} \%$ (54). These data suggest that dietary fat quality is only of importance for insulin sensitivity with a total fat intake equal or lower than $35-40 \%$ of total energy intake. Moreover, in the LIPGENE study, insulin sensitivity significantly improved in women on the high MUFA diet, but only when the habitual fat intake before the start of the study was below $36 \mathrm{En \%}$ (unpublished results). There is also a lot of controversy with respect to the long-term effect of PUFA on insulin sensitivity. A recent meta-analysis showed that n-3 PUFA consumption did not affect insulin sensitivity (57).

Overall, total fat intake alone probably does not have a major effect on insulin sensitivity but the possible interaction between fat quality and quantity needs further investigation. Also heterogeneity in subject characteristics like gender and initial metabolic profile may mask the effect of the dietary intervention. Therefore, a more personalized dietary approach is necessary to increase the intervention success.

\section{Dietary fibers}

Fiber is a major component of carbohydrate that is generally considered beneficial for health and can decrease the energy density of a diet. High fiber foods take longer to eat and have a reduced absorption rate thereby increasing fullness and satiety. They may also stimulate the release of gut peptides that modify eating behavior $(277,278)$. High fiber intake may thus prevent weight gain by reducing energy intake and appetite $(58,59)$. Furthermore, the decreased intestinal passage time and more gradual nutrient absorption may contribute to a reduction in glycemic and insulinemic responses (60-64). These lower glucose and insulin concentrations may, in turn result in a reduced inhibition of lipolysis, higher circulating FFA concentrations and consequently increased fat oxidation $(65,66)$. A greater postprandial fat oxidation may attenuate fat accumulation in non-adipose tissues, leading to an improvement in insulin sensitivity (67). A higher postprandial fat oxidation may also translate in a negative energy balance in the long term. These health-protective effects raised interest of the industry to develop functional fibers, which could be incorporated in food products to replace carbohydrates like glucose and sucrose but with a similar sweetness and taste. However, it still needs to be investigated if these functional fibers deliver the same known health benefits 
as "traditional" fibers. Therefore, in chapter $\mathbf{6}$ we investigated whether replacement of $30 \%$ of the total daily intake of available carbohydrates by polydextrose (PDX) or soluble corn fiber (SCF) at breakfast and lunch would result in an increased fat oxidation rate and satiety, which may be of relevance for body weight control and diabetes prevention. Logically, both fiber diets had a lower caloric value than the full caloric control diet. To correct for the lower caloric value we also compared the PDX diet with a second control diet with the same caloric intake as the PDX diet (iso-caloric control). The full caloric control diet, PDX and SCF diet consisted of approximately the same macronutrient composition in grams. The iso-caloric control diet and PDX diet had approximately the same macronutrient composition in energy percentage. In this way, we could distinguish whether the effects observed were attributed by the fibers per se or the lower caloric content of the fiber diets. Replacing available carbohydrates by PDX and SCF reduced the peak glucose response, which was accompanied by a reduction in postprandial insulin responses. Furthermore, increased circulating FFA concentrations and concomitant a higher fat oxidation were observed. This was mainly attributed by the lower caloric value and the more negative energy balance of the high fiber diets and not the fibers per se. Nevertheless, PDX showed favorable effects on appetite, which appeared to be a specific characteristic of PDX independent of energy balance. Several factors may influence appetite like, the intrinsic physical properties of dietary fiber (bulking, gel formation and viscosity), modulation of gastric motor function, increased mastication, changes in gut hormones (ghrelin or GLP-1) and blunting of postprandial glucose and insulin responses (68-70). Additionally, it has been hypothesised that colonic fermentation resulting in increased production of SCFA could stimulate colonic L-cells to produce several appetite-regulating hormones such as PYY and GLP-1 (70). The responsible mechanisms remain to be elucidated.

The reduced energy content of PDX together with its suppressive effects on appetite may lead to a negative energy balance, which has been shown to increase fat oxidation. The intake of food products in which part of the available carbohydrates is replaced by PDX could be of benefit to individuals aiming to reduce overall energy intake to achieve and maintain a healthy body weight. Furthermore, an increase in fat oxidation may reduce fat accumulation in nonadipose tissues, which may lead to improvements in insulin sensitivity. However, this has to be investigated in a long-term study with ad libitum food intake. Possibly, the energy reduction is unlikely to engender a major reduction in body weight but could contribute to preventing weight gain or weight regain (58). 


\section{Conclusion and future perspectives}

The studies described in this thesis provide insight in the effects of several dietary interventions on body weight control as well as insulin sensitivity by modulation of fat metabolism. The main outcomes and future directions of this thesis are summarized below:

1. A very low calorie diet program induced an initial large decrease in weight, which was accompanied by an improvement in insulin sensitivity. The underlying mechanism for this improvement could not be explained by changes in skeletal muscle fatty acid metabolism related gene expression in the fasted state. This may imply that changes in the postprandial state or other processes possibly play a larger role in lipid-induced insulin resistance. Further research is necessary and should not only focus on skeletal muscle but also explore the other organs in a broader whole-body perspective.

2. Resveratrol supplementation for 30 days reduced adipocyte size and the accompanying gene expression profile hinted towards an improved lipid buffering capacity of the adipose tissue. This together with the improvement in muscle fat oxidative mitochondrial capacity and metabolic flexibility and reduced liver fat accumulation may be underlying mechanisms for the improvement in insulin sensitivity. Studies investigating long-term supplementation of resveratrol are necessary to further explore whether these calorie-restriction like effects remain over time. Secondly, it remains to be determined whether the reduction in energy expenditure, which was observed, will contribute to weight gain making this supplement less attractive for the prevention of metabolic complications.

3. Acute ingestion of a PUFA meal consisting of $n-3$ fish oil and $n-6$ safflower oil (ratio 1:1) has shown to improve postprandial insulin sensitivity compared with an SFA meal. Potential underlying mechanisms for this improvement may be the reduced uptake of TAG-derived FA by the forearm muscle and a higher intramuscular lipid turnover. However, longterm effects of SFA replacement by PUFA on insulin sensitivity may be more complex and may also involve total fat intake. Possibly, also a more personalized dietary approach is preferred to increase the intervention success. 
4. Food products with incorporated functional fibers like PDX and SCF reduced the peak glucose response, which was accompanied by a reduction in postprandial insulin responses. Furthermore, increased circulating FFA concentrations and concomitant a higher fat oxidation were observed. This was mainly attributed by the lower caloric value and the more negative energy balance of the high fiber diets and not by the fibers per se. Nevertheless, PDX showed favorable effects on appetite, which appeared to be a specific characteristic of PDX independent of energy balance. Long-term studies are needed to investigate the effect of these products in an ad libitum setting on body weight control and insulin resistance.

To conclude, the 'ideal' diet for obese insulin resistant subjects should include those dietary components that induce satiating and metabolic properties that are effective for body weight control and improvements in the metabolic profile. A reduction in daily energy intake is usually part of dietary treatment. Increased consumption of food products/food supplements like resveratrol, more unsaturated fatty acids and an increase in certain dietary fibers may improve the metabolic flexibility of fat metabolism, which can contribute to intervention success and long term maintenance of beneficial metabolic effects. However, further research is necessary to evaluate the long-term benefits of these components. Moreover, the study of complete dietary patterns, rather than isolated foods or nutrients, may be the most adequate approach to assess the role of diet on obesity and the insulin resistant state. This because synergistic or antagonistic effects may exist between the different components of the diet. Besides dietary recommendations also regular physical activity should be included in any health promotion and disease prevention strategy. Receiving guidelines about the 'ideal' personal diet should always be in accordance with the persons' own preferences and accompanied by behaviour modifications since the intrinsic motivation of the subject plays an important role to enhance compliance with the diet. Furthermore, governmental actions are needed to modify the food environment. Consumption of unhealthy food should be discouraged by making the costs of it higher and alternatively healthier diets should be promoted by making these products cheaper.

The challenge for the future is to further investigate the link between nutrition and obesity as well as obesity-related health complications to eventually develop the most successful personalized dietary approach to tackle these diseases. 


\section{References}

1. Obesity: preventing and managing the global epidemic. Report of a WHO consultation. World Health Organ Tech Rep Ser 2000;894:i-xii, 1-253.

2. Redman LM, Ravussin E. Caloric restriction in humans: impact on physiological, psychological, and behavioral outcomes. Antioxid Redox Signal 2011;14(2):275-87.

3. Pereira MA, Kottke TE, Jordan C, O'Connor PJ, Pronk NP, Carreon R. Preventing and managing cardiometabolic risk: the logic for intervention. Int $\mathrm{J}$ Environ Res Public Health 2009;6(10):2568-84.

4. Tuomilehto J, Lindstrom J, Eriksson JG, et al. Prevention of type 2 diabetes mellitus by changes in lifestyle among subjects with impaired glucose tolerance. $\mathrm{N}$ Engl $\mathrm{J}$ Med 2001;344(18):1343-50.

5. Corpeleijn E, Feskens EJ, Jansen EH, et al. Improvements in glucose tolerance and insulin sensitivity after lifestyle intervention are related to changes in serum fatty acid profile and desaturase activities: the SLIM study. Diabetologia 2006;49(10):2392-401.

6. Ravussin E, Smith SR. Increased fat intake, impaired fat oxidation, and failure of fat cell proliferation result in ectopic fat storage, insulin resistance, and type 2 diabetes mellitus. Ann N Y Acad Sci 2002;967:363-78.

7. Dresner A, Laurent D, Marcucci M, et al. Effects of free fatty acids on glucose transport and IRS-1-associated phosphatidylinositol 3-kinase activity. J Clin Invest 1999;103(2):253-9.

8. Itani SI, Ruderman NB, Schmieder F, Boden G. Lipid-induced insulin resistance in human muscle is associated with changes in diacylglycerol, protein kinase C, and IkappaB-alpha. Diabetes 2002;51(7):2005-11.

9. Simoneau JA, Veerkamp JH, Turcotte LP, Kelley DE. Markers of capacity to utilize fatty acids in human skeletal muscle: relation to insulin resistance and obesity and effects of weight loss. Faseb J 1999;13(14):2051-60.

10. Blaak EE, Wagenmakers AJ, Glatz JF, et al. Plasma FFA utilization and fatty acid-binding protein content are diminished in type 2 diabetic muscle. Am J Physiol Endocrinol Metab 2000;279(1):E146-54.

11. Debard C, Laville M, Berbe $\mathrm{V}$, et al. Expression of key genes of fatty acid oxidation, including adiponectin receptors, in skeletal muscle of Type 2 diabetic patients. Diabetologia 2004;47(5):917-25.

12. Pender C, Trentadue AR, Pories WJ, Dohm GL, Houmard JA, Youngren JF. Expression of genes regulating malonyl-CoA in human skeletal muscle. J Cell Biochem 2006;99(3):860-7.

13. Mootha VK, Lindgren CM, Eriksson KF, et al. PGC-1alpha-responsive genes involved in oxidative phosphorylation are coordinately downregulated in human diabetes. Nature genetics 2003;34(3):267-73.

14. Patti ME, Butte AJ, Crunkhorn S, et al. Coordinated reduction of genes of oxidative metabolism in humans with insulin resistance and diabetes: Potential role of PGC1 and NRF1. Proceedings of the National Academy of Sciences of the United States of America 2003;100(14):8466-71.

15. Heilbronn LK, Gan SK, Turner N, Campbell LV, Chisholm DJ. Markers of mitochondrial biogenesis and metabolism are lower in overweight and obese insulin-resistant subjects. The Journal of clinical endocrinology and metabolism 2007;92(4):1467-73.

16. Mahoney DJ, Safdar A, Parise G, et al. Gene expression profiling in human skeletal muscle during recovery from eccentric exercise. Am J Physiol Regul Integr Comp Physiol 2008;294(6):R1901-10.17. Wing RR, Blair EH, Bononi P, Marcus MD, Watanabe R, Bergman RN. Caloric restriction per se is a significant factor in improvements in glycemic control and insulin sensitivity during weight loss in obese NIDDM patients. Diabetes Care 1994;17(1):30-6.

18. Klein S, Sheard NF, Pi-Sunyer X, et al. Weight management through lifestyle modification for the prevention and management of type 2 diabetes: rationale and strategies. A statement of the American Diabetes Association, the North American Association for the Study of Obesity, and the American Society for Clinical Nutrition. Am J Clin Nutr 2004;80(2):257-63.

19. Corpeleijn E, Mensink M, Kooi ME, Roekaerts PM, Saris WH, Blaak EE. Impaired skeletal muscle substrate oxidation in glucose-intolerant men improves after weight loss. Obesity (Silver Spring) 2008;16(5):1025-32. 
20. Kelley DE, Goodpaster B, Wing RR, Simoneau JA. Skeletal muscle fatty acid metabolism in association with insulin resistance, obesity, and weight loss. Am J Physiol 1999;277(6 Pt 1):E1130-41.

21. Hernandez-Alvarez MI, Chiellini C, Manco M, et al. Genes involved in mitochondrial biogenesis/function are induced in response to bilio-pancreatic diversion in morbidly obese individuals with normal glucose tolerance but not in type 2 diabetic patients. Diabetologia 2009;52(8):1618-27.

22. Gastaldi G, Russell A, Golay A, et al. Upregulation of peroxisome proliferator-activated receptor gamma coactivator gene (PGC1A) during weight loss is related to insulin sensitivity but not to energy expenditure. Diabetologia 2007;50(11):2348-55.

23. Fabris R, Mingrone G, Milan G, et al. Further lowering of muscle lipid oxidative capacity in obese subjects after biliopancreatic diversion. J Clin Endocrinol Metab 2004;89(4):1753-9.

24. Sadruddin S, Arora R. Resveratrol: biologic and therapeutic implications. J Cardiometab Syndr 2009;4(2):102-6.

25. Halliwell B. Dietary polyphenols: good, bad, or indifferent for your health? Cardiovasc Res 2007;73(2):341-7.

26. Lagouge M, Argmann C, Gerhart-Hines Z, et al. Resveratrol improves mitochondrial function and protects against metabolic disease by activating SIRT1 and PGC-1alpha. Cell 2006;127(6):1109-22.

27. Heilbronn LK, de Jonge L, Frisard MI, et al. Effect of 6-month calorie restriction on biomarkers of longevity, metabolic adaptation, and oxidative stress in overweight individuals: a randomized controlled trial. Jama 2006;295(13):1539-48.

28. Martin CK, Heilbronn LK, de Jonge L, et al. Effect of calorie restriction on resting metabolic rate and spontaneous physical activity. Obesity (Silver Spring) 2007;15(12):2964-73.

29. Doucet E, Tremblay A. Food intake, energy balance and body weight control. Eur $\mathrm{J}$ Clin Nutr 1997;51(12):846-55.

30. Hursel R, Viechtbauer W, Westerterp-Plantenga MS. The effects of green tea on weight loss and weight maintenance: a meta-analysis. Int J Obes (Lond) 2009;33(9):956-61.

31. Dube JJ, Amati F, Stefanovic-Racic M, Toledo FG, Sauers SE, Goodpaster BH. Exerciseinduced alterations in intramyocellular lipids and insulin resistance: the athlete's paradox revisited. Am J Physiol Endocrinol Metab 2008;294(5):E882-8.

32. Meex RC, Schrauwen-Hinderling VB, Moonen-Kornips E, et al. Restoration of muscle mitochondrial function and metabolic flexibility in type 2 diabetes by exercise training is paralleled by increased myocellular fat storage and improved insulin sensitivity. Diabetes 2010;59(3):572-9.

33. Goodpaster BH, He J, Watkins S, Kelley DE. Skeletal muscle lipid content and insulin resistance: evidence for a paradox in endurance-trained athletes. J Clin Endocrinol Metab 2001;86(12):5755-61.

34. Crandall JP, Oram V, Trandafirescu G, et al. Pilot Study of Resveratrol in Older Adults With Impaired Glucose Tolerance. J Gerontol A Biol Sci Med Sci 2012.

35. Brasnyo P, Molnar GA, Mohas M, et al. Resveratrol improves insulin sensitivity, reduces oxidative stress and activates the Akt pathway in type 2 diabetic patients. $\mathrm{Br} \mathrm{J}$ Nutr 2011;106(3):383-9.

36. Ghanim H, Sia CL, Abuaysheh S, et al. An antiinflammatory and reactive oxygen species suppressive effects of an extract of Polygonum cuspidatum containing resveratrol. J Clin Endocrinol Metab 2010;95(9):E1-8.

37. Szkudelska K, Szkudelski T. Resveratrol, obesity and diabetes. Eur J Pharmacol 2010;635(13):1-8.

38. Baur JA, Pearson KJ, Price NL, et al. Resveratrol improves health and survival of mice on a high-calorie diet. Nature 2006;444(7117):337-42.

39. Goossens GH. The role of adipose tissue dysfunction in the pathogenesis of obesity-related insulin resistance. Physiol Behav 2008;94(2):206-18.

40. Singh R, Kaushik S, Wang $\mathrm{Y}$, et al. Autophagy regulates lipid metabolism. Nature 2009;458(7242):1131-5.

41. Settembre C, Di Malta C, Polito VA, et al. TFEB links autophagy to lysosomal biogenesis. Science 2011;332(6036):1429-33.

42. Singh $\mathrm{R}$, Xiang $\mathrm{Y}$, Wang $\mathrm{Y}$, et al. Autophagy regulates adipose mass and differentiation in mice. J Clin Invest 2009;119(11):3329-39.

43. Mariman EC. Human biology of weight maintenance after weight loss. J Nutrigenet Nutrigenomics 2012;5(1):13-25. 
44. Capel F, Klimcakova E, Viguerie N, et al. Macrophages and adipocytes in human obesity: adipose tissue gene expression and insulin sensitivity during calorie restriction and weight stabilization. Diabetes 2009;58(7):1558-67.

45. Siklova-Vitkova M, Klimcakova E, Polak J, et al. Adipose Tissue Secretion and Expression of Adipocyte-Produced and Stromavascular Fraction-Produced Adipokines Vary during Multiple Phases of Weight-Reducing Dietary Intervention in Obese Women. J Clin Endocrinol Metab 2012;97(7):E1176-81.

46. Riserus U, Willett WC, Hu FB. Dietary fats and prevention of type 2 diabetes. Prog Lipid Res 2009;48(1):44-51.

47. Xiao C, Giacca A, Carpentier A, Lewis GF. Differential effects of monounsaturated, polyunsaturated and saturated fat ingestion on glucose-stimulated insulin secretion, sensitivity and clearance in overweight and obese, non-diabetic humans. Diabetologia 2006;49(6):13719.

48. Robertson MD, Jackson KG, Fielding BA, Williams CM, Frayn KN. Acute effects of meal fatty acid composition on insulin sensitivity in healthy post-menopausal women. $\mathrm{Br} J$ Nutr 2002;88(6):635-40.

49. Lopez S, Bermudez B, Pacheco YM, Villar J, Abia R, Muriana FJ. Distinctive postprandial modulation of beta cell function and insulin sensitivity by dietary fats: monounsaturated compared with saturated fatty acids. Am J Clin Nutr 2008;88(3):638-44.

50. Lopez S, Bermudez B, Ortega A, et al. Effects of meals rich in either monounsaturated or saturated fat on lipid concentrations and on insulin secretion and action in subjects with high fasting triglyceride concentrations. Am J Clin Nutr 2011;93(3):494-9.

51. Paniagua JA, de la Sacristana AG, Sanchez E, et al. A MUFA-rich diet improves posprandial glucose, lipid and GLP-1 responses in insulin-resistant subjects. J Am Coll Nutr 2007;26(5):434-44.

52. Perreault L, Bergman BC, Hunerdosse DM, Playdon MC, Eckel RH. Inflexibility in intramuscular triglyceride fractional synthesis distinguishes prediabetes from obesity in humans. Obesity (Silver Spring) 2010;18(8):1524-31.

53. Thomsen C, Storm H, Holst JJ, Hermansen K. Differential effects of saturated and monounsaturated fats on postprandial lipemia and glucagon-like peptide 1 responses in patients with type 2 diabetes. Am J Clin Nutr 2003;77(3):605-11.

54. Vessby B, Uusitupa M, Hermansen K, et al. Substituting dietary saturated for monounsaturated fat impairs insulin sensitivity in healthy men and women: The KANWU Study. Diabetologia 2001;44(3):312-9.

55. Jebb SA, Lovegrove JA, Griffin BA, et al. Effect of changing the amount and type of fat and carbohydrate on insulin sensitivity and cardiovascular risk: the RISCK (Reading, Imperial, Surrey, Cambridge, and Kings) trial. Am J Clin Nutr 2010;92(4):748-58.

56. Tierney AC, McMonagle J, Shaw DI, et al. Effects of dietary fat modification on insulin sensitivity and on other risk factors of the metabolic syndrome--LIPGENE: a European randomized dietary intervention study. Int J Obes (Lond) 2011;35(6):800-9.

57. Akinkuolie AO, Ngwa JS, Meigs JB, Djousse L. Omega-3 polyunsaturated fatty acid and insulin sensitivity: a meta-analysis of randomized controlled trials. Clin Nutr 2011;30(6):702-7.

58. King NA, Craig SA, Pepper T, Blundell JE. Evaluation of the independent and combined effects of xylitol and polydextrose consumed as a snack on hunger and energy intake over 10 d. The British journal of nutrition 2005;93(6):911-5.

59. Wanders AJ, van den Borne JJ, de Graaf C, et al. Effects of dietary fibre on subjective appetite, energy intake and body weight: a systematic review of randomized controlled trials. Obes Rev 2011;12(9):724-39.

60. Biorklund M, van Rees A, Mensink RP, Onning G. Changes in serum lipids and postprandial glucose and insulin concentrations after consumption of beverages with beta-glucans from oats or barley: a randomised dose-controlled trial. European journal of clinical nutrition 2005;59(11):1272-81.

61. Brennan CS. Dietary fibre, glycaemic response, and diabetes. Molecular nutrition \& food research 2005;49(6):560-70.

62. Juntunen KS, Niskanen LK, Liukkonen KH, Poutanen KS, Holst JJ, Mykkanen HM. Postprandial glucose, insulin, and incretin responses to grain products in healthy subjects. The American journal of clinical nutrition 2002;75(2):254-62.

63. Ulmius $M$, Johansson A, Onning $G$. The influence of dietary fibre source and gender on the postprandial glucose and lipid response in healthy subjects. European journal of nutrition 2009;48(7):395-402. 
64. Kendall CW, Esfahani A, Hoffman AJ, et al. Effect of novel maize-based dietary fibers on postprandial glycemia and insulinemia. Journal of the American College of Nutrition 2008;27(6):711-8.

65. Saris WH. Sugars, energy metabolism, and body weight control. The American journal of clinical nutrition 2003;78(4):850S-7S.

66. Brand-Miller J, McMillan-Price J, Steinbeck K, Caterson I. Dietary glycemic index: health implications. Journal of the American College of Nutrition 2009;28 Suppl:446S-9S.

67. Brand-Miller JC, Holt SH, Pawlak DB, McMillan J. Glycemic index and obesity. Am J Clin Nutr 2002;76(1):281S-5S.

68. Papathanasopoulos A, Camilleri M. Dietary fiber supplements: effects in obesity and metabolic syndrome and relationship to gastrointestinal functions. Gastroenterology 2010;138(1):65-72 e1-2.

69. Willis HJ, Eldridge AL, Beiseigel J, Thomas W, Slavin JL. Greater satiety response with resistant starch and corn bran in human subjects. Nutrition research (New York, NY 2009;29(2):100-5.

70. Kristensen M, Jensen MG. Dietary fibres in the regulation of appetite and food intake. Importance of viscosity. Appetite 2011;56(1):65-70. 


\section{Summary}

\section{Samenvatting}




\section{SUMMARY}

The global epidemic of overweight and obesity is rapidly increasing throughout the world. This alarming increase in obesity is associated with a lower age of onset and an increased incidence and prevalence of obesity-related comorbidities like type 2 diabetes mellitus (T2DM). Changes in lifestyle are considered to play an important role in the etiology of obesity and T2DM but there is a need for additional preventive strategies to increase the effectiveness of dietary programs. The identification of food and food ingredients that could influence specific physiological targets like increasing fat oxidation and restoring metabolic flexibility may be helpful in the prevention and treatment of obesity as well as diabetes. Therefore, in this thesis we investigated the effects of several dietary interventions on body weight control as well as the prevention and/or reversal of insulin resistance by modulation of fat metabolism.

The effects of calorie restriction on insulin sensitivity may be mediated by changes in skeletal muscle fatty acid handling but the underlying mechanisms need to be further elucidated. Therefore, in chapter 2 we investigated the effect of a 12-week weight loss intervention on insulin sensitivity and on skeletal muscle fatty acid metabolism-related gene expression in obese subjects with impaired glucose tolerance (IGT). The very low calorie diet induced a large decrease in body weight of approximately $15 \mathrm{~kg}$, which was accompanied by an improvement in insulin sensitivity. No changes in baseline expression of genes involved in muscle fatty acid handling (PGC1 $\alpha$, PPAR $\alpha, A C C-2$, SREBPs and ChREBP) could be observed, despite a tendency towards an increase in muscle oxidative capacity. This may imply that changes in the postprandial state possibly play a larger role in lipid-induced insulin resistance. However, the number of genes determined in the present study was limited. Further research is necessary and should focus on a more complete transcriptomic and functional profile of skeletal muscle but also explore the other organs in a broader whole-body perspective.

Polyphenolic compounds, such as resveratrol are currently an area of intense investigation due to their ability to mimic metabolic effects of calorie restriction. Although originally the focus was mainly directed towards anti-oxidant properties of resveratrol, more recently cellular effects on fatty acid metabolism and mitochondrial function have been described. However, human data were still lacking. Chapter 3 and 4 described the first human study that systematically examined the metabolic effects of resveratrol in healthy obese men. In a randomized cross-over study, resveratrol supplementation (150mg/day) for 30 days clearly improved muscle fat oxidative and mitochondrial capacity and metabolic flexibility. Moreover, resveratrol induced a shift towards an increased proportion of metabolically favorable small adipocytes. This phenotype was accompanied by a 
gene expression profile indicative of increased adipogenesis and an increased lipid breakdown by the alternative pathway of autophagy. Furthermore, intrahepatic lipid content was decreased which may have contributed together with the improved skeletal muscle oxidative capacity to the improvement in insulin sensitivity. Moreover, a $2 \%-4 \%$ reduction in energy expenditure was observed upon resveratrol treatment which may reflect an increased metabolic efficiency and is consistent with the effects observed after calorie restriction. Overall, we can conclude that resveratrol has considerable potential to improve health and prevent chronic disease in humans. However, it is not yet certain if long-term resveratrol supplementation will maintain these physiological benefits. Therefore, further research is necessary to explore whether these calorie-restriction like effects remain over time and if the reduction in energy expenditure, which was observed, will contribute to weight gain making this supplement less attractive for the prevention of metabolic complications.

The underlying mechanisms how dietary fatty acids may affect insulin sensitivity and how the risk or severity of insulin resistance can be modulated by fat quantity and quality are currently under debate. Therefore, in chapter $\mathbf{5}$, the objective was to examine the acute effects of meals with various fatty acid compositions on skeletal muscle fatty acid processing and postprandial insulin sensitivity in obese, insulin-resistant men using the dual-stable fatty acid isotope technique in a randomized cross-over design. With this technique, the contribution of dietary fat (chylomicron-TAG, CM-TAG) vs. endogenous fat (FFA and VLDL-TAG) to in vivo skeletal muscle fatty acid metabolism could be investigated by using two different labels. [U- ${ }^{13} \mathrm{C}$-palmitate was added to a high fat mixed meal to label CM-TAG in the circulation and $\left[{ }^{2} \mathrm{H}_{2}\right]$-palmitate was infused intravenously to label the endogenous pool of fatty acids. Acute ingestion of a high fat mixed meal, high in polyunsaturated fatty acids (PUFA) consisting of $n-3$ fish oil and $n-6$ safflower oil (ratio 1:1) improved postprandial insulin sensitivity compared with a high saturated fatty acid (SFA) meal. Potential underlying mechanisms for this improvement may be the reduced uptake of TAG-derived fatty acids by the skeletal muscle, a higher incorporation of dietary $\left[\mathrm{U}-{ }^{13} \mathrm{C}\right.$-palmitate from the muscle FFA pool in the different lipid fractions (higher fractional synthetic rate [FSR]) in skeletal muscle and a lesser downregulation of the mitochondrial oxidative genes. These data indicate an impaired muscle fatty acid handling and turnover of in particular SFA. However, long-term effects of SFA replacement by PUFA on insulin sensitivity may be more complex and may also involve total fat intake.

Fiber is a major class of carbohydrates and has been reported to result in several health effects. High fiber intake may decrease glycaemic and insulinemic responses, which may favour lipolysis and fat oxidation rather than storage and may attenuate fat accumulation in non-adipose tissues leading to improved insulin sensitivity. A higher postprandial fat oxidation may also translate in a negative 
energy balance in the long term. This has led to industrial interest in developing dietary fibers that can be incorporated in a variety of food products. However, the question remains if these fibers provide the same health benefits as traditional dietary fibers. For that reason, we studied in chapter 6 in a randomized crossover study, whether replacement of $30 \%$ of the total daily intake of available carbohydrates by polydextrose (PDX) or soluble corn fiber (SCF) at breakfast and lunch would result in an increased fat oxidation rate and satiety in eighteen overweight men and women. This may be of relevance for body weight control and diabetes prevention. Logically, both fiber diets had a lower caloric value than the full caloric control diet. To correct for the lower caloric value we also compared the PDX diet with a second control diet with the same caloric intake as the PDX diet (iso-caloric control). In this way, we could distinguish whether the effects observed were attributed by the fibers per se or the lower caloric content of the fiber diets. A diet with incorporated functional fibers like PDX and SCF reduced the peak glucose response, which was accompanied by a reduction in postprandial insulin responses. Furthermore, increased circulating FFA concentrations and concomitant a higher fat oxidation were observed. This was mainly attributed by the lower caloric value and the more negative energy balance of the high fiber diets and not the fibers per se. Nevertheless, PDX showed favorable effects on appetite, which appeared to be a specific characteristic of PDX independent of energy balance. Long-term studies are needed to investigate whether the positive short term effects of these products translate into long term benefits on body weight control and insulin resistance in an ad libitum setting. Possibly, the energy reduction is unlikely to engender a major reduction in body weight but could contribute to preventing weight gain or weight regain.

It is obvious that the 'ideal' diet for obese insulin resistant subjects should include those dietary components that induce health benefits and satiating properties that are effective for body weight control and improvements in insulin sensitivity. Increased consumption of food products/food supplements like resveratrol, more unsaturated fatty acids and an increase in certain dietary fibers may improve the metabolic flexibility of fat metabolism, which may contribute to intervention success and long term maintenance of beneficial metabolic effects. However, further research is necessary to evaluate the long-term benefits of these components. Moreover, the study of complete dietary patterns, rather than isolated foods or nutrients, may be the most adequate approach to assess the role of diet on obesity and the insulin resistant state. This is because synergistic or antagonistic effects may exist between the different components of the diet. The challenge for the future is to further investigate the link between nutrition and obesity as well as obesity-related health complications to eventually develop the most successful personalized dietary approach to tackle these diseases. 


\section{SAMENVATTING}

De epidemie van overgewicht en obesitas verspreidt zich in snel tempo over de hele wereld. Deze alarmerende toename van obesitas is geassocieerd met een verhoogde incidentie en prevalentie van co-morbiditeiten zoals type 2 diabetes mellitus (T2DM). Bovendien komt overgewicht op steeds jongere leeftijd voor, waarbij veranderingen in leefstijl een belangrijke rol spelen. Het volgen van een dieet kan hulp bieden, maar er is duidelijk behoefte aan extra preventieve strategieën om de effectiviteit van dieet programma's te verhogen. Onderzoek naar bepaalde voedingsmiddelen en voedselingrediënten die fysiologische processen kunnen beïnvloeden zoals het verhogen van de vetverbranding en het herstellen van metabole flexibiliteit, kan een belangrijke rol spelen bij de preventie en behandeling van obesitas en T2DM. In dit proefschrift werd onderzocht wat de effecten waren van verschillende voedingsinterventies op het lichaamsgewicht en het voorkomen en/of omkeren van insuline resistentie door het beïnvloeden van de vetstofwisseling.

De effecten van calorie restrictie op de insulinegevoeligheid kan worden gemedieerd door veranderingen in de vetstofwisseling van de skeletspier, maar de onderliggende mechanismen moeten verder worden onderzocht. Daarom hebben we in hoofdstuk 2 het effect bestudeerd van een 12 weken durend dieet op insuline gevoeligheid en op genen betrokken bij de vetstofwisseling in de skeletspier bij obese proefpersonen met een verstoorde glucose tolerantie (IGT). Het dieet was gebaseerd op een zeer lage inname van calorieën wat leidde tot een grote daling in het lichaamsgewicht van ongeveer $15 \mathrm{~kg}$ en een verbetering van de insulinegevoeligheid. $\mathrm{Er}$ werden geen veranderingen waargenomen in verschillende genen betrokken bij de vetstofwisseling (PGC1 $\alpha$, PPAR $\alpha$, ACC-2, SREBPs en ChREBP) in de skeletspier in nuchtere toestand. Desondanks trad er toch een verbetering op in de verbrandingscapaciteit van de spier. Dit kan betekenen dat veranderingen in de postprandiale toestand een grotere rol spelen in lipide-geïnduceerde insulineresistentie. Het aantal genen dat we bepaald hebben in deze studie was echter gelimiteerd. Verder onderzoek is noodzakelijk en moet zich meer richten op het volledige transcriptoom en het functionele profiel van de spier, maar moet daarnaast ook de andere organen hierbij betrekken om alles in een breder perspectief te kunnen zien.

Polyfenolen zoals resveratrol kunnen de metabole effecten van calorie restrictie nabootsen. Hoewel oorspronkelijk de aandacht gericht was op de antioxidantwerking van resveratrol is er recentelijk meer aandacht voor het effect van resveratrol op de vetstofwisseling en de mitochondriële functie. In hoofdstuk 3 en 4 hebben we de eerste humane studie beschreven die systematisch de metabole effecten van resveratrol heeft onderzocht bij gezonde obese mannen. In 
een gerandomiseerde cross-over studie waarbij resveratrol $(150 \mathrm{mg} / \mathrm{dag})$ werd toegediend gedurende 30 dagen verbeterde de verbrandingscapaciteit van vet in de mitochondriën van de spier en de metabole flexibiliteit. Daarnaast was er ook een toename in de proportie van kleine, metabool gunstige vetcellen. Dit fenotype ging gepaard met een genexpressieprofiel dat wees op een verhoogde adipogenese en een alternatieve pathway van vetafbraak (autofagie). Verder werd een verminderde vetstapeling in de lever gevonden die samen met een efficiëntere omzetting van vetten in de spier kan hebben geleid tot de verbetering in insulinegevoeligheid. Verder was er een reductie in energieverbruik van $2 \%-4 \%$ na resveratrol supplementatie wat overeenkomt met effecten die aangetoond zijn na calorie restrictie en wat kan wijzen op een verhoogde metabole efficiëntie. Uit deze resultaten kunnen we concluderen dat resveratrol potentieel heeft om de gezondheid te verbeteren en chronische ziekten te voorkomen. Het is echter niet zeker of deze gunstige fysiologische effecten behouden kunnen worden op lange termijn. Verder onderzoek is nodig om te onderzoeken of de vermindering in energieverbruik die waargenomen werd, zal bijdragen aan een gewichtstoename, wat dit supplement minder aantrekkelijk zou kunnen maken als preventief middel tegen metabole complicaties.

De onderliggende mechanismen die verklaren hoe vetten in de voeding de insuline gevoeligheid kunnen beïnvloeden en hoe de hoeveelheid en de kwaliteit van het vet het risico op de ontwikkeling van insulineresistentie kunnen beïnvloeden, staan momenteel nog ter discussie. Het doel van hoofdstuk 5 was om de acute effecten van maaltijden rijk aan vet maar met verschillende vetzuursamenstellingen op de vetstofwisseling in de skeletspier en de postprandiale insulinegevoeligheid te onderzoeken bij obese, insulineresistente mannen. Dit werd gedaan met behulp van twee verschillende isotopen van het vetzuur palmitaat in een gerandomiseerde cross-over studie. Met deze techniek werd de bijdrage van vetten uit de voeding (chylomicron-triacylglycerol, CM-TAG) of vetten reeds aanwezig in het lichaam (vrije vetzuren en VLDL-TAG) onderzocht. [U- ${ }^{13} \mathrm{C}$-palmitaat werd toegevoegd aan een gemengde maaltijd rijk aan vetten om de CM-TAG in de circulatie te labelen en $\left[{ }^{2} \mathrm{H}_{2}\right]$-palmitaat werd intraveneus geïnfuseerd om de endogene pool van vetzuren te labelen. De acute inname van een maaltijd met meervoudig onverzadigde vetten (PUFA) die bestond uit $n-3$ visolie en $n-6$ saffloerolie (ratio 1:1) verbeterde de postprandiale insuline gevoeligheid in vergelijking met een maaltijd met verzadigde vetten. Dit ging gepaard met een verminderde opname van vetzuren afkomstig van triglyceriden door de spier, een hogere opname van voedingsvetzuren in de verschillende lipide fracties van de spier (hogere fractionele synthetische snelheid) en een verminderde downregulatie van mitochondriële oxidatieve genen in vergelijking met de maaltijd met veel verzadigd vet. Deze gegevens wijzen op een hogere lipiden turnover na inname van PUFA in vergelijking met verzadigde vetten, wat één van de verklaringen kan zijn voor de verhoogde postprandiale insuline 
gevoeligheid. Echter, het effect van het vervangen van verzadigde vetten door meervoudig onverzadigde vetten op de insulinegevoeligheid op de lange termijn is complex en mogelijk speelt hier de totale vetinname ook een belangrijke rol.

Vezels zijn een belangrijke component van koolhydraten en kunnen bijdragen aan positieve gezondheidseffecten. Een hoge inname van vezels kan de glucose- en insulinerespons doen afnemen en kan zo bijdragen aan een verbeterde afbraak en oxidatie van vet en verminderde vetopslag. Hierdoor zullen er minder vetten stapelen in ectopische weefsels wat kan leiden tot een betere insulinegevoeligheid. Een hogere postprandiale vetverbranding kan zich ook vertalen in een negatieve energiebalans op de lange termijn. Hierdoor is de interesse van de industrie gewekt om vezels te produceren die toegevoegd kunnen worden aan verschillende voedingsmiddelen. De vraag is echter of deze vezels dezelfde positieve effecten bieden voor de gezondheid als de traditionele voedingsvezels. Om die reden onderzochten we in hoofdstuk 6 in een gerandomiseerde cross-over studie of de vervanging van $30 \%$ van de totale dagelijkse inname van beschikbare koolhydraten door polydextrose (PDX) of oplosbare maïsvezels (SCF) bij het ontbijt en de lunch zou resulteren in een verhoogde vetverbranding en verzadiging bij achttien mannen en vrouwen met overgewicht. Dit zou het lichaamsgewicht onder controle kunnen houden en diabetes kunnen voorkomen. Beide vezel diëten hadden een lagere calorische waarde dan het dieet met de controle voedingsmiddelen. Om te corrigeren voor deze lagere calorische waarden werd het PDX dieet ook vergeleken met een tweede controle dieet met hetzelfde aantal calorieën (iso-calorische controle). Op deze manier kon er worden onderzocht of de effecten door de vezels zelf veroorzaakt werden of door de lagere calorische inhoud van de vezel diëten. Een dieet met deze functionele voedingsvezels verminderde de piekwaarde van de postprandiale glucoserespons, wat gepaard ging met een vermindering in postprandiale insulinerespons. Bovendien namen de circulerende vrije vetzuurconcentraties toe en gelijktijdig werd een hogere postprandiale vetverbranding waargenomen. Dit werd voornamelijk veroorzaakt door de calorische waarde en de meer negatieve energiebalans van het vezelrijke dieet en niet door de vezels zelf. Desalniettemin vertoonde PDX gunstige effecten op de eetlust, wat onafhankelijk was van de energiebalans. Er zijn echter lange termijn studies nodig om het effect van deze producten te onderzoeken op de regulatie van het lichaamsgewicht en de insulinegevoeligheid in een ad libitum setting. Waarschijnlijk zal de reductie in energie geen groot effect hebben op het verlies in lichaamsgewicht maar kan het wel bijdragen aan de preventie van gewichtstoename en het jojo-effect.

Het is duidelijk dat het "ideale" dieet voor obese insulineresistente mensen dient te bestaan uit die voedingscomponenten die een gezond voedingspatroon induceren en verzadigende eigenschappen bezitten die effectief zijn voor verlaging en handhaving van het lichaamsgewicht en die verbeteringen in insuline gevoeligheid 


\section{Samenvatting}

induceren. Verhoogde consumptie van voedingsmiddelen/ voedingssupplementen zoals resveratrol, meer onverzadigde vetzuren en een toename in bepaalde voedingsvezels kunnen de metabole flexibiliteit van het vetmetabolisme verbeteren en kunnen zo bijdragen aan leefstijlinterventie succes en lange termijn handhaving van gunstige metabole effecten. $\mathrm{Er}$ is echter verder onderzoek nodig om de langetermijn effecten van deze componenten te evalueren. Bovendien moet het onderzoek gericht zijn op het bestuderen van volledige voedingspatronen, in plaats van losse voedingsmiddelen of nutriënten, om zo het effect van voeding op obesitas en insulineresistentie vast te stellen. Dit omdat er synergetische of antagonistische effecten kunnen bestaan tussen de verschillende componenten van het dieet. De uitdaging voor de toekomst is het verband tussen voeding en obesitas, alsook obesitas gerelateerde ziektes verder te onderzoeken om uiteindelijk het meest succesvolle persoonlijke dieet te ontwikkelen om deze ziektes te voorkomen of aan te pakken. 


\section{Acknowledgments}

\section{Dankwoord}




\section{Coming together is a beginning, keeping together is progress, working together is \\ success! \\ $\sim$ Henry Ford}

Een dankwoord schrijven geeft altijd een beetje een dubbel gevoel. Enerzijds een gevoel van opluchting omdat het proefschrift klaar is en ik dit hoofdstuk eindelijk kan afsluiten om me ten volle over te geven aan nieuwe uitdagingen die op mijn pad liggen. Anderzijds een gevoel van gemis omdat ik hier 4 jaar intensief mee bezig ben geweest en naast de minder leuke dingen ook onvergetelijke momenten heb beleefd! Daarom wil ik graag iedereen bedanken die op wat voor manier dan ook heeft bijgedragen tot dit succes!

Daarnaast verdienen nog een aantal mensen een speciaal woordje van dank.

Allereerst wil ik mijn promotoren Prof. dr. Ellen Blaak en Prof. dr. Edwin Mariman bedanken voor de mogelijkheid die ik heb gekregen om een promotie onderzoek te starten.

Beste Ellen, je deur stond altijd open en ik kon steeds bij je terecht met al mijn vragen. Ik bewonder hoe je telkens de positieve kanten van mijn onderzoek naar boven wist te brengen.

Beste Edwin, bedankt voor al de keren dat je zomaar even kwam binnenspringen om een praatje te maken. Door deze gesprekken kreeg ik weer extra moed om er keihard tegenaan te gaan en heb ik meer inzicht gekregen in het onderzoek. Bedankt allebei voor de steun gedurende de afgelopen jaren en voor de talloze revisies van de hoofdstukken en manuscripten om zo dit proefschrift naar een hoger niveau te brengen.

Next, I would like to thank all members of the reviewing committee, Prof. dr. Wim Saris, Prof dr. Karine Clément, dr. Jogchum Plat, Prof. dr. Casper Schalkwijk and Prof. dr. Annemie Schols for taking the time to review my thesis and/or being present at the official dissertation.

Anneke en Annemieke, de keuze was snel gemaakt om jullie als mijn paranimfen te kiezen. Sinds de eerste dag bij HB had ik het geluk om bij jullie op de kamer te mogen. Anneke, wat was het leuk om samen onze eerste humane studie te mogen uitvoeren. We waren echt een superteam! Daarnaast hebben we ook nog vele fijne 
carpoolmomenten beleefd. Ik wens je nog het allerbeste toe en nog veel geluk met je gezinnetje in jullie nieuwe huis!

Annemieke, wat vonden we het jammer toen Anneke bij ons weg ging. Het laatste jaar hebben we dus met ons 2-tjes doorgebracht. Dit betekende echter niet dat het minder gezellig was. Ik kon altijd bij je terecht al was het maar om af en toe eens te klagen ;-) Ik wens je nog veel geluk in je verdere leven met Christiaan en hoop dat we nog lang in contact blijven. Ook bedankt voor de laatste aanpassingen aan de kaft. Hij is nu echt geworden zoals ik in gedachten had!

Met ons 3-en hebben we veel plezier gemaakt waardoor Ellen B. zich soms afvroeg of er ook nog ooit werd gewerkt :-). Zo hielden we ons onder andere bezig met lachwekkende photoboot momenten, het oefenen van de belt flip (en dat in een overvolle kamer), jongleer oefeningen (braintraining...), het verzamelen van $\mathrm{AH}$ en Plus gadgets, de snoeppot vullen en leeg eten (na een tijdje zijn we hier maar mee gestopt in het kader van een gezonde leefstijl), bloemetjes zaaien, zelf kleine experimenten opzetten, in de zomer ijsjes eten, lunchwandelingen, een tocht door Maastricht om spullen te kopen voor het vezelonderzoek, het in leven proberen houden van ons plantje dat bijna dood was doordat we alle drie in dezelfde periode op vakantie gingen, en nog meer van deze ontspannende activiteiten... Maar wees niet ongerust daarnaast werd er ook keihard gewerkt! Kortom, we hebben samen zoveel beleefd dat ik niemand anders wilde als paranimfen om mij bij mijn promotie bij te staan!

Chantalle, je hebt Anneke en mij de kneepjes van het vak geleerd. Zonder jou was onze eerste studie niet zo een succes geweest. Ik zal nooit vergeten hoe goed jij met de proefpersonen om kon gaan om het ze naar hun zin te maken. Het eindeloos aantal cupjes schrijven en altijd opnieuw dezelfde films meekijken viel uiteindelijk erg mee omdat het altijd zo gezellig was! Ook bedankt om deel te nemen aan één van mijn studies.

Silvie, jij verdient ook een speciaal woordje van dank. De tweede humane studie mocht ik namelijk met jou uitvoeren. Voor jou was het je eerste humane studie en je hebt dit echt super goed aangepakt! Ondanks de vele weekends (inclusief nachten) die je op de uni hebt doorgebracht bleef je op maandag nog tot in de late uurtjes tot de testdag was afgelopen. Ik bewonder je doorzettingsvermogen en je wetenschappelijk inzicht! Het was erg fijn om met je te mogen samenwerken!

Gijs, bedankt voor de ondersteuning bij de vele humane studies! Je stond altijd klaar om te helpen met het plaatsen van infusen en probes en het afnemen van spierbiopten. We konden altijd op je rekenen. Ook bedankt voor de vele wetenschappelijke en minder wetenschappelijke discussies, dit heeft zeker bijgedragen tot mijn inzicht in het onderzoek. Veel succes in je verdere loopbaan! 
Johan, je bent voor mij een echte diehard wetenschapper! Met al mijn labvragen kon ik steeds bij jou terecht. Hoe dikwijls heb ik niet bij je op de kamer gestaan als een western blot weer niet lukte. Je had steeds weer nieuwe ideeën om tot een oplossing te komen. Ik hoop dat je nog vele kansen krijgt om jouw briljante ideeën uit te werken want je verdient het om hierin verder te gaan!

Anneke VH en Eefje, de "oude garde" van het vetstofgroepje! Eefje, ook al had je een nieuwe baan in het verre Groningen, ik kon steeds bij jou terecht met al mijn vragen. Anneke $\mathrm{VH}$, toen ik begon was $\mathrm{jij}$ in de afrondfase van je promotie, jij was voor mij de expert in de stabiele isotopendata en jouw templates zijn voor velen van ons nog goed van pas gekomen!

Dorien, Jasper, Emanuel en Rudi, de "nieuwe" garde van het vetstofgroepje! Bedankt voor de leuke babbels en wetenschappelijke discussies. Veel succes nog met jullie onderzoek en Dorien laat je niet doen door al die mannen ;-)

Hoewel we op het einde niet meer een gezamenlijk overleg hadden met het groepje van Marleen wil ik ook graag jullie bedanken! Roel, Erik, Dorit en Karianna bedankt voor de collegialiteit! Karianna nog eens extra bedankt om altijd voor me klaar te staan als ik weer een vraag had over een statistische analyse. Je bent het statistisch wonder van $\mathrm{HB} ;-)$

Buurmannen Herman en Jonathan, bedankt voor de gezellige gesprekken, leuke practica en het printen van mijn labels. Wat was ik blij toen ik niet meer al die cupjes met de hand moest schrijven! Het heeft me heel wat tijd en werk bespaard!

Judith, Marjet en Paul, bedankt om mij te introduceren in de wondere wereld van de respiratiekamer. Paul, als er iets mis was met de respiratiekamer stond je steeds weer voor me klaar, zelfs in het weekend en de avonduren! Judith en Marjet, dankzij jullie gingen mijn respiratiekamer metingen erg vlot omdat ik het van de experts geleerd had ;-) Ook bedankt voor de gezellige avonden als we samen een meting hadden!

Doordat ik een Mac en PC had, betekende het soms ook dat de problemen voor twee telden. Loek, bedankt dat jij voor elk probleem wel een oplossing wist!

Laurens, ook jou mag ik niet vergeten. Als er iets was met de omnical, diskettes die niet werkten (ja die bestaan nog bij HB) of bestanden die op mysterieuze wijze niet wilden openen op mijn computer stond jij altijd voor me klaar. 
Claudia, Desiree en Jolanda, bedankt voor de hulp als ik mijn sleutel weer eens vergeten was, met het fax- en scanapparaat en de administratie rond mijn proefschrift.

Ook bedankt aan alle analisten, Wendy, Hasibe, Annemie, Yvonne en Jos voor het uitvoeren van de vele analyses. Zonder jullie was het nooit gelukt! Nog een speciaal woordje van dank aan Yvonne en Jos!

Yvonne, bedankt voor de hulp bij de analyses van de vet- en spierbiopten. Ik heb erg veel van jou geleerd!

Jos, wat was het gezellig als je zoals gewoonlijk 's avonds en in het weekend op het werk was terwijl ik een respiratiekamermeting had! Hierdoor voelde ik me tenminste niet alleen en had ik iemand om samen mee te eten, TV kijken en af en toe een biertje (kriek Lindemans ;-)) te drinken. Hoewel de bitterballen helaas niet meer mochten, zijn de borrels nog steeds een succes! Bedankt Jos, om de organisatie steeds weer op jou te nemen en om iedereen te motiveren om op vrijdag om $16 \mathrm{u}$ het werk neer te leggen en weekend te vieren!

Freek, Montserrat, Anja, Anke, Antoine, Johan S. en Johan R., bedankt voor de gezelligheid op het lab tijdens het runnen van de vele PCR's en western blots. Jullie stonden steeds voor me klaar als ik weer een vraag had of een helpende hand nodig had.

Dit proefschrift is mede tot stand gekomen door de samenwerking met Wageningen voor het runnen van de micro-arrays. Bedankt Michaël, Mark en Lydia voor de hartelijke ontvangst en de goede wetenschappelijke discussies. Ik heb zeer veel van jullie geleerd tijdens de week dat ik bij jullie mocht werken om me te verdiepen in de micro-array analyses. Al hadden we in het begin vele hindernissen te overwinnen dit weerhield me niet om bij een volgende studie nog eens voor deze techniek te kiezen $\odot$

Humane studies uitvoeren kan je niet zonder proefpersonen. Bedankt allemaal!

Bedankt aan alle collega's voor de leuke congressen, het ontspannende citytripje Istanbul, gezellige borrels, de fijne vakgroepuitjes, weekendje Ardennen en zoveel meer waardoor ik weer extra energie kreeg om verder te gaan.

I also want to thank my new colleagues at Medtronic for the pleasant atmosphere and for making me feel very welcome. Although we are located all over the world we are really one team! It is great working with you all!

Er zijn uiteraard ook mensen die ervoor gezorgd hebben dat het leven niet alleen uit onderzoek en het proefschrift bestond. Allereerst "de jeugd" van de harmonie. 
Ook al hebben sommigen onder ons een niet meer zo jeugdige leeftijd toch noemen we ons nog steeds zo ;-) Door de leuke sfeer was ik meestal snel vergeten dat ik op zondagochtend weer zo vroeg moest opstaan om klarinet te spelen. Daarnaast hebben de vele uitstapjes (dag en nacht), repetitieweekenden en de reis naar Praag voor onvergetelijke momenten gezorgd! Ik hoop dus nog vele jaren bij de "jeugd " te mogen horen. Nog een extra bedankje voor Sanne! Zonder jou had ik nu niet zo een mooie kaft, het was erg leuk om samen het voorbereidende werk te doen!

Ook bedankt aan iedereen van het Biomedische clubje. Ook al zie ik sommigen van jullie meer dan anderen toch is het steeds gezellig als we weer een keertje afspreken. Ik hoop dus dat we dit in de toekomst nog vaak kunnen doen. Kathleen, ook al heeft ons Vietnamreisje misschien voor enige vertraging gezorgd in het afmaken van mijn proefschrift. Ik heb er zeker geen spijt van want het was een reis om nooit te vergeten!

Nele en Sarah, de etentjes en spelletjesavonden waren altijd zeer gezellig en nu dat mijn proefschrift eindelijk klaar is heb ik terug meer tijd om vaker naar Leuven af te zakken.

Het frisbeeteam, ook al was ik meestal meer actief naast het veld (als supporter) dan op het veld, ik heb erg genoten van de vele tornooien in binnen- en buitenland in de zon en de regen en zal de leuke verkleedfeestjes niet snel vergeten!

Ook het jaarlijkse weekendje met de "oud klasgenoten" van Fery en de verscheidene activiteiten van A.S.G. hebben voor de nodige ontspanning gezorgd!

Familie en schoonfamilie, sommigen onder jullie vroegen zich af wanneer ik nu eindelijk klaar zou zijn met studeren of wisten nooit helemaal precies wat ik deed. Toch hebben jullie steeds voor de nodige interesse, afleiding en plezier gezorgd!

Mama en papa, ik neem aan dat jullie nog steeds niet goed begrijpen waar ik eigenlijk de voorbije vier jaar mee bezig ben geweest. Desondanks hebben jullie alles nauwlettend op de voet gevolgd en steeds geïnteresseerd geluisterd naar wat ik te vertellen had. Ik wil jullie graag danken omdat jullie er steeds voor me zijn en omdat ik altijd op jullie kan rekenen!

Als allerlaatste dan eindelijk de persoon die mijn dank het meeste heeft verdiend en die ik waarschijnlijk niet genoeg kan bedanken. Fery, mijn liefje, er is niemand die deze periode van zo dichtbij heeft meegemaakt. Je was er altijd wanneer ik je het meeste nodig had! Bedankt voor het nalezen van mijn thesis en een welgemeende sorry voor al die keren dat ik je heb verwaarloosd. Het proefschrift is nu eindelijk af en ik verheug me enorm op de periode die nu komen zal met meer tijd voor elkaar om samen te genieten! Uit het diepst van mijn hart: dankjewel voor al je liefde en geduld. Ik hou van jou helemaal tot aan de maan en terug! 
Curriculum Vitae

187 


\section{Curriculum Vitae}

Ellen Konings was born on November $6^{\text {th }} 1985$ in Hasselt, Belgium. She started secondary school at Lyceum in Hasselt where she specialised in sciences and maths. After her graduation in 2003, she started with her bachelor Molecular Life Sciences at the transnational University Limburg, on the campus located in Diepenbeek, Belgium. She graduated in 2006 and continued with the MSc program Clinical and Molecular Life Sciences at the same university. She did her master thesis entitled 'Characterisation of Endothelial Progenitor Cells' at the Jessa hospital in Hasselt, Belgium. In August 2007, she started working as a lab technician at the Stem Cell Institute Leuven of the K.U.Leuven, Belgium. In September 2008, she started her PhD at the department of Human Biology of the Maastricht University Medical Center. The research performed during this project, under supervision of Prof dr. Ellen E. Blaak and Prof. dr. Edwin C. Mariman, is described in this thesis entitled 'Dietary manipulation of fat metabolism in relation to obesity and insulin resistance'.

In September 2012, she started working as Associate Clinical Quality Specialist at Medtronic, Maastricht, The Netherlands. 


\section{List of publications}




\section{Published Manuscripts}

Knop FK, Konings E, Timmers S, Schrauwen P, Holst JJ, Blaak EE. 30 days of resveratrol supplementation does not affect postprandial incretin hormone responses, but suppresses postprandial glucagon in obese subjects. Diabetic Medicine In Press.

Jans $A^{*}$, Konings $E^{*}$, Goossens $G H$, Bouwman FG, Moors CCM, Boekschoten $M V$, Afman LA, Müller M, Mariman EC, Blaak EE. Polyunsaturated fatty acids acutely affect triacylglycerol-derived skeletal muscle fatty acid uptake and increases postprandial insulin sensitivity. Am J Clin Nutr. 2012 Apr;95(4):825-36.

Timmers $\mathrm{S}^{*}$, Konings $\mathrm{E}^{*}$, Bilet L, Houtkooper $\mathrm{RH}$, van de Weijer T, Goossens $\mathrm{GH}$, Hoeks J, van der Krieken S, Ryu D, Kersten S, Moonen-Kornips E, Hesselink MKC, Kunz I, Schrauwen-Hinderling VB, Blaak EE, Auwerx J, Schrauwen P. Calorie restriction-like effects of 30 days of resveratrol (resVida ${ }^{\top \mathrm{M}}$ ) supplementation on energy metabolism and metabolic profile in obese humans. Cell Metab. 2011 Nov 2;14(5):612-22.

Goossens GH, Bizzarri A, Venteclef N, Essers Y, Cleutjens JP, Konings E, Jocken JW, Cajlakovic M, Ribitsch V, Clément K, Blaak EE. Increased adipose tissue oxygen tension in obese compared with lean men is accompanied by insulin resistance, impaired adipose tissue capillarization, and inflammation. Circulation. 2011 Jul 5;124(1):67-76.

Konings E, Corpeleijn E, Bouwman FG, Mariman EC, Blaak EE. Expression of genes involved in lipid metabolism in men with impaired glucose tolerance: impact of insulin stimulation and weight loss. J Nutrigenet Nutrigenomics. 2010;3(1):9-17.

\section{Manuscripts in preparation}

Konings $\mathrm{E}^{*}$, Timmers $\mathrm{S}^{*}$, Boekschoten MV, Goossens GH, Jocken JW, Afman LA, Müller M, Schrauwen P, Mariman EC, Blaak EE. The effects of 30 days resveratrol supplementation on adipose tissue morphology and the underlying processes in obese men. Submitted.

Konings E, Schoffelen PF, Stegen J, Blaak EE. The effect of polydextrose and soluble corn fiber on energy metabolism, metabolic profile and appetite control in overweight men and women. Submitted.

Moors CCM, Goossens GH, van der Zijl NJ, Jans A, Konings E, Diamant M, Blaak EE. Different skeletal muscle fatty acid handling in subjects with impaired fasting glucose and impaired glucose tolerance. Submitted.

* shared first authorship 
